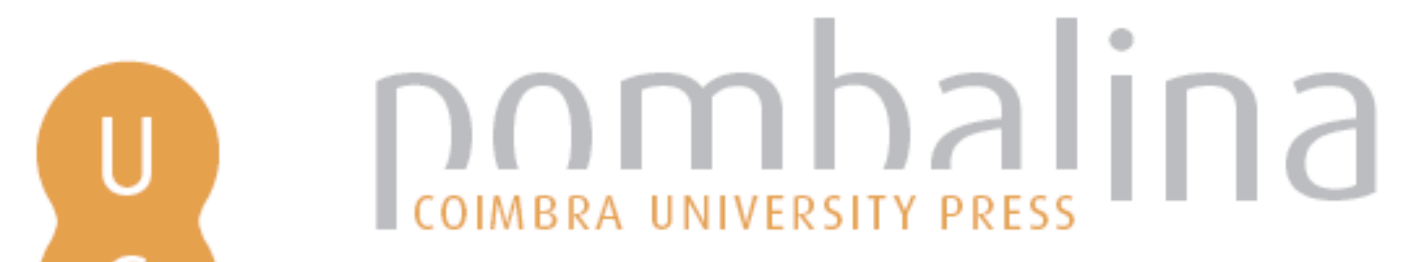

\title{
Cognition et émotions
}
Autor(es):
Kirouac, Gilles, ed. lit.
Publicado por: $\begin{aligned} & \text { Imprensa da Universidade de Coimbra; Les Éditions de L'IQRC (Les } \\ & \text { Presses de l'Université Laval) }\end{aligned}$
URL
persistente:
URI:http://hdl.handle.net/10316.2/2566
DOI:
DOI:https://doi.org/10.14195/978-989-26-1587-5
Accessed : $\quad$ 26-Apr-2023 15:29:06

A navegação consulta e descarregamento dos títulos inseridos nas Bibliotecas Digitais UC Digitalis, UC Pombalina e UC Impactum, pressupõem a aceitação plena e sem reservas dos Termos e Condições de Uso destas Bibliotecas Digitais, disponíveis em https://digitalis.uc.pt/pt-pt/termos.

Conforme exposto nos referidos Termos e Condições de Uso, o descarregamento de títulos de acesso restrito requer uma licença válida de autorização devendo o utilizador aceder ao(s) documento(s) a partir de um endereço de IP da instituição detentora da supramencionada licença.

Ao utilizador é apenas permitido o descarregamento para uso pessoal, pelo que o emprego do(s) título(s) descarregado(s) para outro fim, designadamente comercial, carece de autorização do respetivo autor ou editor da obra.

Na medida em que todas as obras da UC Digitalis se encontram protegidas pelo Código do Direito de Autor e Direitos Conexos e demais legislação aplicável, toda a cópia, parcial ou total, deste documento, nos casos em que é legalmente admitida, deverá conter ou fazer-se acompanhar por este aviso.

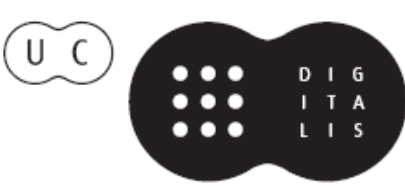




\section{GILLES KIROUAC}

\section{Coordonnateur Scientifique}

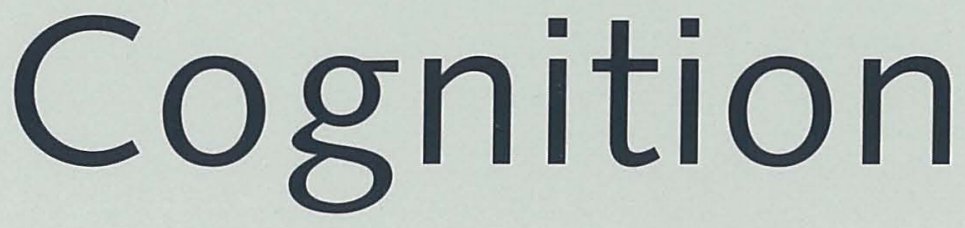

$$
\text { et }
$$

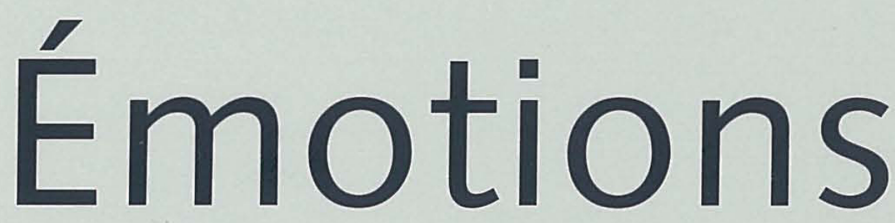


(Página deixada propositadamente em branco) 


\title{
GILLES KIROUAC
}

Coordonnateur Scientifique

\section{Cognition et Émotions}

\author{
AUTEURS \\ Klaus R. Scherer \& Janique Sangsue \\ Pierre Philippot \\ Dario Galati \\ Bernard Rimé \\ Pierre Gosselin \\ Ursula Hess \\ Arvid Kappas \& Jean Descôteaux \\ Pio E. Ricci Bitti \\ Pedro Luzes \\ Lise Fillion \\ Ramon Bayés \\ Coimbra - Imprensa da Universidade


COORDENAÇÃO EDITORIAL

Imprensa da Universidade de Coimbra

CONCEPÇÃO GRÁFICA

António Barros

PAGINAÇÃo

António Resende

[Universidade de Coimbra]

EXECUÇÃO GRÁFICA

G.C. - Gráfica de Coimbra, Lda.

Palheira - Assafarge - Apart. 3068

300I-453 Coimbra Codex

ISBN 972-8704-19-4

ISBN 2-7637-81 I8-7

ISBN DIGITAL 978-989-26-1587-5

DOI https://doi.org/10.14195/978-989-26-1587-5

DEPÓsITO LEGAL $210430 / 04$

(C) MAIO 2004, IMPRENSA DA UNIVERSIDADE DE COIMBRA

OBRA PUBLICADA COM O PATROCINIO DO

INSTITUTO DE PSICOLOGIA COGNITIVA

FCT: FUNDAÇÃO PARA A CIÊNCIA E A TECNOLOGIA

MINISTÉRIO DA CIÊNCIA E DO ENSINO SUPERIOR

APOIO DO PROGRAMA OPERACIONAL CIENCIA, TECNOLOGIA, INOVAÇÃO

DO QUADRO COMUNITÁRIO DE APOIO 


\section{TABLE DES MATIÈRES}

Avant-Propos ....................................................................................................................... 5

Préface ............................................................................................................................................ 7

Klaus R. Scherer \& Janique Sangsue

Le système mental en tant que composant de l'émotion .......................................... I I

Pierre Philippot

Facteurs cognitifs et réactions corporelles dans le processus émotionnel.........

Dario Galati

Le langage des émotions: leur donner une voix ou les mettre en paroles?....

Bernard Rimé

Émotion et mémoire: la rémanence des expériences émotionnelles

79

Pierre Gosselin

La reconnaissance de l'expression faciale des émotions.

Ursula Hess

Émotion ressentie et simulée

Arvid Kappas \& Jean Descôteaux

Les promesses et limites de l'étude de l'émotion en laboratoire

Pio E. Ricci Bitti

La régulation des comportements expressifs émotionnels.

Pedro Luzes

L'Interaction cognition-émotion et la Psychologie Clinique.

Lise Fillion

Émotion, cognition et immunité.

Ramon Bayés

Perception des symptômes et réactions émotionnelles lors des maladies mortelles. 
(Página deixada propositadamente em branco) 


\section{AVANT-PROPOS}

Les travaux scientifiques réunis dans ce volume portent sur les processus de médiation et d'interaction entre les activités cognitives et les émotions déclenchées par différentes situations ou conditions de vie. II s'agit d'un champ de recherche d'une actualité incontournable dont l'intérêt théorique et pratique dépasse le domaine de la Psychologie pour se situer à un niveau interdisciplinaire. En effet, au-delà des études centrés sur l'éclaircissement de problèmes classiques, comme ceux qui concernent la nature, l'origine et la séquence des facteurs (physiologiques et psychologiques) des émotions ou bien comme ceux qui visent à comprendre le rôle de l'expression faciale ou linguistique des expériences émotionnelles, ce volume intègre aussi des études qui visent à mettre en évidence les rapports entre les émotions, les activités cognitives et des processus générateurs de maladies et de souffrance. C'est le cas des recherches concernant les rapports entre les expériences émotionnelles et les altérations du système immunitaire. Les résultats qui en découlent ouvrent le champ nouveau de la psycho-neuro-immunologie, dont le développement mobilise des efforts articulés de différentes disciplines. Mais c'est aussi le cas des recherches sur les rapports entre la perception des symptômes et les réactions émotionnelles lors des maladies mortelles, telles la peur, l'anxiété, la souffrance et la douleur. Ces études, de plus en plus nombreuses, aident à élargir le domaine multidisciplinaire des soins palliatifs, un domaine de recherche et d'intervention pratique qui est encore assez jeune mais dont l'essor, dans le prochain avenir, deviendra de plus en plus accru pour pouvoir correspondre à son importance psycho-médico-sociale.

Les travaux de recherche scientifique publiés dans ce volume ont fait objet d'une présentation préliminaire lors des XXVes Journées d'Études de l'Association de Psychologie Scientifique de Langue Française (APSLF), tenues 
à Coimbra, en Septembre de 1995. Depuis lors, ils ont été enrichis par des actualisations fondées sur des recherches conduites par leurs propres auteurs et sur la prise en compte des résultats obtenus par d'autres chercheurs.

En acceptant le défi de publier pour la première fois un livre en langue française, l'Imprensa da Universidade de Coimbra a voulu correspondre, tout d'abord, au projet de contribuer à l'élargissement de l'espace de sa diffusion, assuré par la diversité des nationalités d'origine des auteurs des différents chapitres. Mais le motif majeur de l'acceptation de ce défi repose, sans doute, sur l'actualité scientifique et sur l'importance pratique et multidisciplinaire des matières analysées dans ce volume. Nous voulons adresser nos plus vifs remerciements au Professeur Gilles Kirouac, de I'Université de Laval, pour son effort en tant que coordinateur scientifique de cette édition, et au Professeur Manuel Viegas Abreu, de I'Université de Coimbra, par la persistance qu'il a su maintenir dès l'organisation des XXVes Journées d'Études de l'Association de Psychologie Scientifique de Langue Française à la concrétisation du projet de diffusion des travaux présentés. Notre gratitude s'adresse également à toutes les institutions dont l'appui a permis la mise en oeuvre de cette publication. Nous voulons remercier, particulièrement, à l'Instituto de Psicologia Cognitiva de la Faculté de Psychologie et des Sciences de l'Education de l'Université de Coimbra, attaché à la Fundação para a Ciência e a Tecnologia du Ministère de la Science et de l'Enseignement Supérieur portugais, au moyen du «Programme Science, Technologie et Innovation» intégré dans le Quadre d'Appui de la Communauté Européenne.

Coimbra, Mai de 2004

Fernando Regateiro

Directeur de l'Imprensa da Universidade de Coimbra 


\section{PRÉFACE}

Pendant très longtemps (Hilgard, 1980), I'analyse psychologique a organisé son étude du fonctionnement mental humain en le divisant en grandes facultés. Parmi ces dernières, on retrouve, en particulier, la cognition et l'émotion (affection ou affectivité). II reste à déterminer les relations qui existent entre ces grandes entités. À ce propos, dès 1980, Hilgard a montré que, dans une forte mesure, la psychologie cognitive contemporaine tend à englober ce qui, historiquement au moins, apparait comme des aspects séparés au sein des mécanismes de contrôle du comportement. C'est le cas tout particulièrement de l'émotion. D'ailleurs, il importe de mentionner que, à la même époque, Norman (1980) estimait qu'une des questions fondamentales auxquelles devait répondre la science cognitive pour mieux accomplir son rôle, portait justement sur la nature de l'émotion. II ne faut donc pas s'étonner que l'un des problèmes les plus actuels en psychologie de l'émotion s'avère justement la nature des relations entre l'émotion et la cognition.

D'un autre côté, il y a une vieille controverse concernant les mécanismes responsables du déclenchement de l'expérience émotionnelle. Cette controverse s'est historiquement cristallisée autour de la prise de position de William James (1884) qui propose une séquence précise des facteurs responsables de la présence d'une émotion. II affirme que, contrairement au sens commun, les changements corporels propres à l'émotion viennent à la suite de la perception de l'objet évocateur de l'émotion et que la prise de conscience de ces changements, à mesure qu'ils se produisent, constitue l'émotion ou plutôt l'expérience émotionnelle. Cet énoncé théorique, proposé simultanément par Lange (Lange et James, 1967), se révèle la pierre angulaire de ce qu'il est convenu d'appeler, en psychologie de l'émotion, le problème de la séquence. Les débats autour de cette question ont 
considérablement marqué l'évolution de la recherche en psychologie de l'émotion.

En fait, une question fondamentale qui recoupe l'ensemble de la problématique venant d'être soulevée consiste à se demander si une activité cognitive quelconque (souvent appelée évaluation) doit obligatoirement précéder l'activation de tout état émotionnel. En d'autres termes, le problème qui se pose n'est pas tant de savoir si la cognition est une cause suffisante de l'émotion, mais plutôt si elle constitue une cause nécessaire. À ce sujet, les opinions ont divergé fortement. Certains croient qu'il est théoriquement pertinent de considérer que des patrons déterminés d'activité neurophysiologique peuvent générer l'émotion de façon indépendante de l'analyse cognitive. À ce moment-là, ils estiment que l'information afférente peut se transformer directement en émotion sans médiation cognitive ; ces auteurs font alors appel à l'intervention de mécanismes non conscients ou préconscients de traitement des stimuli affectifs. Par contre, d'autres prétendent que la genèse de l'émotion dépend toujours de mécanismes cognitifs, notamment d'évaluation: l'émotion constitue alors un phénomène qui est la résultante d'une interaction entre des changements physiologiques et des processus psychologiques ou encore de l'intervention exclusive de l'analyse cognitive.

La profusion des recherches à saveur «cognitiviste», tout particulièrement celle sur l'évaluation cognitive (voir en particulier Scherer, Schorr et Johnstone, 200I), oblige à reconnaitre le caractère prédominant de cette approche en psychologie de l'émotion. Les variables cognitives ont récemment acquis un statut empirique de plus en plus consolidé et leur impact se traduit en particulier par un foisonnement de conceptions théoriques. Quoi qu'il en soit, compte tenu de l'importance actuelle du courant cognitiviste, il faut aussi mentionner un autre débat qui a occupé très activement les spécialistes de l'émotion en même temps que se consolidaient les théories cognitives : il s'agit de savoir si l'émotion est une activité psychologique complètement dominée par la cognition ou si elle possède une indépendance réelle. II s'agit, en considérant essentiellement le système mental humain, de se demander si l'émotion ou le système actif constitue une entité psychologique distincte et autonome par rapport à la cognition.

Tout d'abord, il faut se demander de quelle manière, du point de vue des définitions respectives, on pourrait situer l'émotion et la cognition en 
faisant appel à des différences susceptibles d'être définies opérationnellement. De fait, ces différences, et la relation émotion-cognition qui les accompagne, peuvent être considérées sous l'angle de deux conceptions distinctes. La première postule l'existence d'un seul système qui possède des fonctions différentes et mesurables. À ce moment-là, l'émotion constitue un facteur au sein de la cognition et du traitement de l'information. Par contre, la seconde conception propose la présence de deux systèmes séparés. Ces derniers seraient fortement en interaction et, possiblement, interdépendants. II y aurait alors un système émotionnel séparé qui traiterait de l'information dite «affective». Un tel système influencerait le comportement indépendamment des processus cognitifs.

II y a plusieurs années, Izard, Kagan et Zajonc (1984) ont montré que la séparation des mécanismes cognitifs et émotionnels en deux systèmes a des conséquences importantes pour une composante centrale de l'émotion, c'est-à-dire l'expérience subjective. Ainsi si cette dernière se distingue de la cognition, il reste alors à analyser les relations de cet état avec la cognition et à préciser de quelle façon les sentiments s'associent ou interagissent avec les images et les symboles. Cette conception implique aussi que l'expérience subjective doit se définir comme comportant plusieurs composantes conscientes: les sentiments émotionnels mais aussi les images, les souvenirs et tous les autres produits du traitement de l'information. Quant à ceux qui estiment que l'expérience subjective émotionnelle est essentiellement de nature cognitive, il leur faut préciser ce qui constitue la composante cognitive de l'émotion par rapport aux autres produits des processus cognitifs. De plus, ils doivent aborder la question de la distinction entre la cognition portant sur l'émotion et la cognition qui est une composante de l'état émotionnel lui-même. Cette question revient à se demander comment l'identification et la représentation symbolique de l'émotion sont reliées à l'expérience subjective de l'émotion. Toutes ces questions posées par lzard et ses collaborateurs (1984) sont présentes depuis longtemps en psychologie de l'émotion. II n'y a guère de réponses unanimes à ces problèmes.

Le débat portant sur la relation entre émotion et cognition perdure donc depuis plusieurs années étant donné la vigueur des arguments avancés par les protagonistes, l'absence de résultats empiriques suffisamment concluants et la confusion terminologique qui entoure souvent la question. Compte tenu du foisonnement de prises de position sur la question de la relation entre cognition et émotion et des nombreuses ramifications qu'elle a 
suscitées, l'Association de psychologie scientifique de langue française a décidé de consacrer ses XXVe journées d'études à cette passionnante problématique. Réunis à Coimbra au Portugal, du 14 au 16 septembre 1995, plusieurs experts dans le domaine ont exposé leur point de vue sur une variété d'aspects liés à cette très importante question qui est au centre des études sur les émotions. Le présent ouvrage est un recueil de la majorité des exposés faits à l'occasion de ces journées d'études. Leur lecture permettra de mieux apprécier la richesse des thématiques que renferme cette question.

\author{
Gilles Kirouac
}

Le coordonnateur scientifique

\title{
RÉFÉRENCES
}

Hilgard, E.R. (1980). The trilogy of mind: Cognition, afffection and conation. Journal of the History of the Behavorial Sciences, 16, 107-117.

Izard, C.E., Kagan, J. \& Zajonc, R.B. (1984). Emotions, Cognition and Behavior. Cambridge: Cambridge University Press.

James, W. (1884). What is an emotion? Mind, 9, 188-205.

Lange, C.G. \& James, W. (1967). The Emotions. New York: Hafner. (Réimpression de textes publiés en 1884, 3n 1885 et en 1890).

Norman, D.A. (1980). Twelve issues for cognitive science. In A.A. Norman (Ed.), Perspectives on cognitive science: Talks from the La Jolla Conference (pp. 3-I4). Hillsdale, NJ : Erlbaum. Scherer, K.R., Schorr, A. and Johnstone, T. (200I). Appraisal Processes in Emotion. Oxford : Oxford University Press. 
Klaus R. Scherer \& Janique Sangsue

Université de Genève, Suisse

\section{LE SYSTĖME MENTAL EN TANT QUE COMPOSANT DE L'ÉMOTION}

Le domaine de l'émotion reste encore actuellement l'enfant pauvre des ouvrages de psychologie en langue française. La dernière contribution de taille fut celle de Fraisse (1969) dans son Traité de Psychologie Expérimentale, contribution qui cependant ne constituait qu'un résumé encyclopédique des diverses définitions de l'émotion. Ceci parait surprenant puisque la tradition francophone, avec Dumas, Janet, Pradines, Wallon ou Malrieu, s'est considérablement penchée sur l'étude du phénomène émotionnel en abordant l'un ou l'autre de ses aspects (physiologique, comportemental, affectif ou social).

II nous a semblé opportun de resituer ces auteurs et leur contribution particulière à la théorie des émotions. Des remarques émises ci-dessous, il ressort que le caractère adaptatif de l'émotion, reconnu maintenant unanimement, faisait l'objet de débats passionnés au début du XXème siècle. Les controverses étaient liées à des ambiguités profondes, l'une terminologique, relevant des définitions respectives de l'émotion et du sentiment, l'autre portant sur l'origine et la nature de l'émotion et, partant, sur l'affrontement entre thèses intellectualiste et physiologique.

\section{L'émotion: l'équivoque d'une définition et sa conséquence}

Le concept d'émotion a souffert au début du siècle dernier d'un manque de clarté dans l'emploi respectif des termes émotion, sentiment, passion et états affectifs, ceux-ci étant assimilés l'un à l'autre ou employés l'un pour l'autre en fonction de leur utilisateur. En insistant sur le rôle primordial du sentiment dans l'adaptation, l'émotion était dotée d'un revers négatif: elle 
est une désorganisation et une désadaptation. En vertu de sa particularité quantitative, son intensité, elle a été perçue comme une forme explosive de l'affectivité qui, en envahissant le champ de la conscience, provoque un retour aux automatismes préformés. Elle est une intensité «en plus», «en trop» dans le comportement humain ou encore le «raté d'une régulation sentimentale» (Pradines, 1954).

Si la proposition suivante: «Réaction organisée et utile à une situation donnée» apparaît définitionnelle de l'émotion pour la plupart des théoriciens contemporains, elle correspondait chez Janet à la notion de sentiment. En effet, Janet (1926), puis également Pradines (1954) octroient au sentiment, non à l'émotion, une fonction régulatrice, un caractère fonctionnel et adaptatif. Selon ces auteurs, le sentiment est ce qui nous donne à l'égard des objets une règle d'action parfaitement adéquate; il a pour rôle de mettre notre psychisme en harmonie avec les circonstances, de l'orienter en fonction de ce qu'elles nous offrent et de nous inspirer des conduites efficaces. Wallon, Malrieu ou encore Bergson vont plus loin, en associant le phénomène émotionnel au développement psychique et à la régulation des comportements sociaux.

\section{Une nécessité adaptative: l'émotion et sa nature sociale}

Les doctrines de Wallon (1934, 1938) et de Malrieu (1952) ont évoqué principalement le contexte social et interactionnel associé au phénomène émotionnel. Pour Wallon, dès les premiers jours de vie de l'enfant, les réactions motrices et les attitudes posturales font naître des émotions auxquelles le milieu est appelé à répondre. Ce sont d'elles que procèdent les premières régulations du comportement, puis les progrès des habitudes motrices. La valeur fonctionnelle des émotions chez Wallon tient à ce qu'elles permettent de superposer aux automatismes la diversité des réactions affectives comme moyens d'expression. Selon Malrieu également, leur fonctionnalité s'exprime au travers de leur action sur autrui.

Ainsi, bien loin d'être de simples réactions désorganisatrices, les émotions sont au contraire décisives pour l'adaptation de l'individu et ceci, dès sa naissance. Elles continuent à l'être, par la suite, en tant qu'adaptations circonstanciées à des modèles sociaux. Pour Wallon ou Malrieu, la société exerce une influence sur la différenciation et la reconnaissance des émotions, 
sur leur expression et leur gestion interindividuelle. D'une part, l'acteur social doit accorder ses expressions émotionnelles aux impressions qu'il souhaite produire, en vertu de «codes sociaux», l'idéal étant qu'il y ait harmonie entre les éprouvés, leur expression et les impressions produites. D'autre part, il y a l'ordre social qui impose une dialectique de l'expression émotionnelle et de son usage, tant public que privé. C'est ce dernier point que plusieurs ouvrages classiques français du début du siècle dernier ont traité pour retracer le rapport entre le social et l'émotion, mais leurs discussions des codes émotionnels a principalement concerné l'expression des émotions.

Pour Dumas (1948a), les mots « expression émotionnelle » sont psychologiquement vides de sens et ne peuvent se comprendre que si l'on suppose une vie sociale où l'expression sera interprétée. Par exemple, le sourire figure un geste dont nous pouvons nous servir quand nous le croyons utile, en imitant par des mouvements volontaires un mouvement réflexe. Les expressions émotionnelles, et les émotions, ne sont pas uniquement ce qu'elles sont pour nous, mais aussi ce qu'elles sont pour autrui et leur signification dépend de l'accueil qu'elles reçoivent.

Tout un chapitre de l'Encyclopédie La Pléiade a été également consacré à la nature sociale de l'émotion, retenant que la société fonde son système d'attitudes affectives et détermine ainsi la valeur qu'une communauté attribue aux différents états affectifs. Par exemple, le rire y est défini comme un fait humain institutionnalisé, c'est-à-dire obéissant à des prescriptions sociales, à obligations et des interdits, de même qu'à des exigences relationnelles (voir Clapier-Valadon, 1991). Henri Bergson (1940) avait déjà insisté sur ces particularités du rire, dans son ouvrage intitulé «Le Rire». Ce philosophe indique que, pour comprendre le rire, il faut le replacer dans son milieu naturel de production qui est la société; le rire est un geste social: il est humour, moquerie, ironie.... et poursuit un but utile, celui de «perfectionnement de la société», parce qu'il fixe une réglementation humaine, en informant les individus de ce qu'il faut faire en cas d'émotion.

\section{Origine et nature de l'émotion: l'âme et le corps \& l'opposition entre thèses intellectualistes et physiologiques}

Dès l'aube des premiers propos sur les émotions, deux thèses sont nées et se sont affrontées, l'une partisane d'une origine purement mentale des 
émotions et l'autre, adepte d'une origine organique. Pour la première, les émotions sont dérivées et fonction de la connaissance, pour la seconde, elles ont leur racine dans les besoins et les instincts. Le problème devient que, ce faisant, il y a toujours réduction des émotions en états organiques ou intellectuels, impliquant toujours le sacrifice d'un des facteurs et l'impossibilité d'expliquer leurs rapports. Cet état de fait brouille la discussion du caractère adaptatif vs. non adaptatif de l'émotion.

Dans la théorie de Dumas (1900, 1948ab), cependant, apparaissent un dépassement de cette dichotomie et une prise en compte du rapport âme-corps en tant qu'unité vécue, ce qui justifie, en partie au moins, la plaidoirie de celui-ci en faveur du caractère adaptatif de l'émotion. Vu l'état des connaissances en son temps, Dumas a fourni un travail impressionnant pour caractériser les états organiques, dans leurs aspects physiologiques, psycho-chimiques et psycho-mécaniques, durant l'émotion. Cet auteur reconnaît que les faits physiologiques n'ont pas de valeur explicative suffisante des phénomènes émotionnels et que c'est une erreur de méconnaitre la signification et l'efficacité des facteurs proprement psychiques. A cheval entre thèse physiologique et thèse intellectualiste, Dumas définit l'émotion comme se scindant en deux temps: un moment de surprise et un moment d'émotion spéciale. La surprise, c'est le choc produit par ce qui est nouveau et inattendu auquel répondent des modifications organiques, physiologiques, et, au niveau des manifestations motrices, des mouvements organisés remplaçant réflexes et mouvements automatiques. Parallèlement à ce désordre physique s'instaure un désordre mental: il y a convergence de la pensée vers la cause de ce trouble. Une représentation va intervenir entre le fait nouveau et les habitudes particulières que ce fait nouveau désorganise. Aux représentations négatives, relatives à la désorganisation, se mêlent des représentations positives, à la recherche d'orientations nouvelles des habitudes physiques et des habitudes mentales gênées. Dans cette perspective, l'émotion autorise des variations adaptatives ou des adaptations nouvelles, puisqu'elles modifient les instincts et les habitudes, physiques et mentales.

Dans cet article, nous défendrons l'idée que l'émotion peut être considérée comme un processus de composants qui englobe tous les sous-systèmes organiques et qui est principalement dirigé par des changements dans l'évaluation de l'information reçue. Ce point de vue est incompatible avec une conception de l'existence de systèmes cognitif et affectif séparés, une idée que nous appellerons l'erreur de Platon. II est également 
incompatible avec l'opinion selon laquelle il est un nombre limité d'émotions de base ou fondamentales, ayant chacune un pattern spécifique de réponses physiologiques et expressives. Ces deux incompatibilités seront exprimées sous forme de propositions et étayées par des arguments conceptuels ainsi que des données empiriques.

\section{Les émotions sont différenciées par un traitement évaluatif de l'information dans le système mental. Il est peu probable qu'il y ait un système de laffect séparé}

Dans son modèle tripartite de l'âme, Platon a postulé l'existence de modèles structurels parallèles pour l'esprit humain et la société, en mettant l'accent sur:

(I) une rigide séparation entre les classes sociales et, en conséquence, les composants de l'âme,

(2) la supposition d'un antagonisme entre ces composants, et

(3) la suprématie éthique de la cognition et donc de la classe des philosophes/rois.

Ce modèle propose que le comportement est fortement affecté par trois déterminants: un élément appétitif ou impulsif, un élément de pensée et de raison et, entre les deux, un élément capable de réfréner les impulsions et désirs et capable également de recevoir des ordres de la pensée et de la raison. Cet élément intermédiaire était appelé «thumos», interprétable comme un facteur de l'émotion spirituelle la plus idéale se manifestant en tant que ressentiment, et contre les transgressions d'autrui, et contre celles de nos propres appétits. II convient de noter que ce composant du modèle de Platon, plus tard généralisé à l'émotion ou l'affect en général, était à l'origine réduit à une fonction de «chien de garde» au service de la sagesse et du jugement rationnel. Le bien-être personnel, selon Platon, suppose un fonctionnement harmonieux, rationnellement coordonné de ces éléments (impulsif, cognitif et «thumos»).

Ce modèle n'était pas universellement accepté et a attiré de virulentes critiques, même de la part des élèves de Platon, tel Aristote. Néanmoins, il a influencé virtuellement tout penseur qui s'est depuis lors intéressé à l'esprit humain. II exerce encore quelque influence sur la théorie et la recherche en psychologie actuelle, comme cela peut être mis en évidence par les controverses récentes sur les relations entre cognition et émotion. 
Pour notre part, nous soutiendrons que la distinction platonicienne entre cognition, émotion et conation est dépassée dans son utilité et peut gêner sérieusement, en plus de provoquer de futiles débats, les progrès vers des approches intégratives dont il se fait pour l'heure un besoin urgent pour l'étude de l'activité mentale.

Dans le contexte d'un modèle de processus composants, Scherer (1984a,b,c, 1986, 1993b, 2000, 200 I) a suggéré de définir l'émotion comme un épisode de synchronisation temporaire des principaux sous-systèmes du fonctionnement organique représenté par cinq composants (cognition, régulation physiologique, motivation, expression motrice et sentiment) en réponse à l'évaluation d'un stimulus, externe ou interne, comme pertinent aux intérêts centraux de l'organisme. Bien que les différents sous-sytèmes ou composants opèrent relativement indépendamment les uns des autres durant des épisodes non-émotionnels, en ce qui concerne leur fonction respective dans la régulation globale du comportement, ils travaillent à l'unisson dans des situations d'urgence, les épisodes émotionnels.

L'épisode émotionnel est ainsi conçu comme débutant avec la mise en route de la synchronisation s'ensuivant d'une évaluation du stimulus particulier et cessant avec le retour à un fonctionnement indépendant de ces sous-systèmes (bien que ces systèmes diffèrent dans leur rapidité de réponse et de traitement). Puisque l'on s'attend à ce que l'évaluation du stimulus affecte chaque sous-système directement et compte tenu que tous ces sous-systèmes sont définis comme étant hautement interreliés durant l'épisode émotionnel, la régulation est complexe et englobe de nombreux processus rétro- et pro-actifs.

Pour plusieurs raisons, le sentiment subjectif pourrait figurer le composant le plus important de l'émotion. Nous pourrions en effet nous demander si nous pouvons prétendre avoir une émotion si nous n'avons pas une expérience consciente de celle-ci, un sentiment spécifique que nous pouvons ensuite étiqueter avec un concept verbal approprié. II semble raisonné de considérer le composant sentiment comme une réflexion des changements se produisant dans tous les sous-systèmes (voir Scherer, 1993a, 200 I). Ces sous-systèmes gagnent en effet leur signification principalement à travers l'interprétation des processus d'évaluation cognitive.

Cependant, dans la supposition d'un traitement «cognitif», il reste hautement probable que tous les processus d'évaluation ne soient pas accessibles à la conscience. En effet, certaines des réflexions des systèmes 
neurophysiologique et moteur, aussi bien que les changements de motivation, peuvent ne pas être complètement accessibles à la mémoire. Il est donc nécessaire de distinguer grossièrement dans le sentiment une part inconsciente et une part consciente constituant la représentation globale des facteurs intervenant dans un épisode émotionnel (c'est-à-dire le traitement cognitif des antécédents et les réactions suscitées dans les différentes modalités). On peut par-là même supposer que la part consciente des sentiments va au-delà du matériel inconscient rendu conscient. En raison d'un traitement cognitif actif et constructif, elle pourrait être partiellement construite sur la base de schémas, scripts et représentations sociales. Donc, comme montré dans la Figure I (adapté de Kaiser \& Scherer, 1998), nous pouvons représenter ces parties du composant sentiment par deux cercles de Venn se recoupant partiellement.

Un troisième cercle, chevauchant en partie les deux autres, semble indiqué pour représenter la verbalisation de la partie consciente du sentiment. A nouveau, l'usage d'étiquettes linguistiques ou d'expressions pour décrire la part consciente du sentiment ne couvrira pas toute la zone consciente, notamment du fait de l'absence de labels verbaux appropriés. Un tel usage ajoutera un surplus de sens, c'est-à-dire un ajout de contenu, vue la signification dénotationnelle et connotationnelle des concepts utilisés dans la verbalisation. Ces aspects de signification qui seraient fonction des règles du langage pourraient ne pas toujours être complètement appropriés à l'état de sentiment conscient.

L'approche par les cercles de Venn conduit donc à 7 aires ou aspects du sentiment:

(I) un chevauchement total de l'évaluation inconsciente et consciente verbalisées de manière appropriée;

(2) une représentation inconsciente est consciemment représentée mais ne peut être verbalisée;

(3) une évaluation inconsciente est intuitivement verbalisée sans être consciemment verbalisée;

(4) une représentation consciente construite, basée sur aucune évaluation inconsciente, est verbalisée - le cas typique du stéréotype;

(5) une évaluation inconsciente reste inaccessible;

(6) une évaluation consciente construite n'est pas verbalisée;

(7) un surplus de sens est fourni par l'usage d'étiquettes verbales, non basé sur une représentation consciente. 

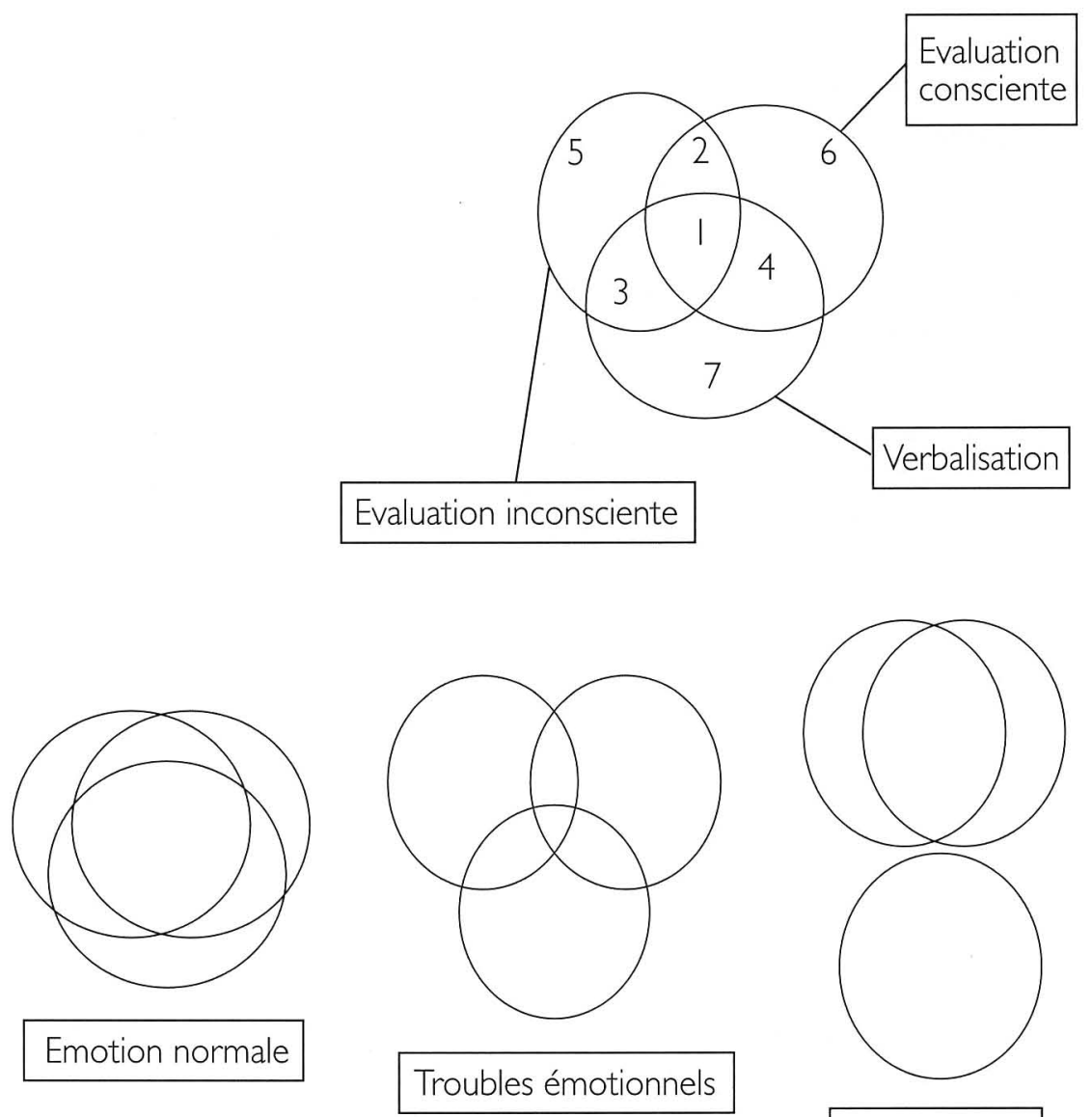

Alexithymie

Figure I - Aires du sentiment, illustrées par les cercles de Venn

Sous des conditions normales, les trois aires du sentiment, illustrées pour des émotions normales dans la Figure I par les cercles de Venn, tendent à se recouper plutôt fortement. En d'autres termes, une grande part du traitement émotionnel est accessible à la conscience, en particulier l'évaluation cognitive d'événements pertinents, donnant une signification appropriée à l'expérience affective, et une grande part peut être adéquatement verbalisée aux fins de communication sociale. 
Les désordres affectifs pourraient être caractérisés par une dissociation augmentée de ces aspects du sentiment. En effet, les processus d'évaluation qui ont généré un état émotionnel particulier pourraient rester inaccessibles à la conscience, du fait de répression ou autres mécanismes de défenses, de déficits attentionnels, de malfonctionnements neurologiques ou d'autres facteurs. Le surplus de signification de la représentation consciente du sentiment pourrait donc être également déterminé dans une large mesure par des facteurs non liés aux processus d'évaluation réels, comme dans le cas d'idées compulsives apparaissant sous formes de schémas ou scripts que la personne tiendrait concernant le soi ou l'environnement social et/ou physique. De même, la verbalisation pourrait représenter seulement une très petite partie du composant sentiment conscient et donc être considérée comme bizarre et inappropriée par l'environnement social, comme par exemple dans le cas d'alexithymie, cité pour exemple de désordre émotionnel (voir Figure I, ainsi que Kaiser \& Scherer, 1998).

\section{Approche fonctionnelle des processus émotionnels}

Le modèle proposé adopte une approche fonctionnelle, en tentant de prendre son point de départ dans les systèmes mental, physiologique et comportemental dont on peut montrer leur asservissement à l'adaptation comportementale - ce qui s'intègre fort dans l'esprit de Darwin. Dans ce sens, la cognition - ou plus généralement le codage de l'information reçue (voir LeDoux, 1989) - joue un rôle déterminant dans la mise en accord des autres sous-systèmes organiques. Ceci n'est pas surprenant puisqu'une adaptation adéquate demande, à tout le moins, un diagnostic approprié des circonstances auxquelles il convient de s'adapter.

L'émotion peut être considérée comme l'une des réalisations principales de l'évolution dans le sens qu'elle remplace la chaîne instinctive Stimulus-Réponse (qui demande seulement un traitement rudimentaire des stimuli déclenchés de façon innée (Lorenz, 1965)) par un mécanisme hautement flexible qui «découple» le stimulus et la réponse. Le processus émotionnel fournit ainsi un temps de latence qui permet un traitement de l'information toujours plus sophistiqué et un choix toujours plus vaste d'alternatives comportementales, mais en préparant néanmoins l'organisme à des réponses d'urgence grossièrement appropriées. 
Le rôle du traitement évaluatif de l'information pour rendre compte de la nature adaptative de l'émotion a été implicitement accrédité par les plus éminents penseurs de l'émotion, tels Aristote, Descartes, Hume et Spinoza. Cependant, la notion d'évaluation (《 appraisal ») comme étant à la racine du déclenchement et de la différenciation de l'émotion est beaucoup plus récente. Dans les années 60, Arnold (1960) et Lazarus (1968) ont explicitement formulé des théories incorporant des critères d'évaluations rudimentaires pour expliquer les conséquences émotionnelles de la confrontation à un événement particulier. Au début des années 80 , plusieurs psychologues ont proposé, indépendamment les uns des autres, des ensembles plus détaillés de dimensions d'évaluation pour interpréter le déclenchement et la différenciation de l'émotion (de Rivera, 1977; Frijda, 1986; Johnson-Laird, \& Oatley, 1989; Mees, 1985; Ortony, Clore, \& Collins, 1988; Roseman, 1984, 1991; Smith \& Ellsworth, 1985, 1987; Scherer, 1981, 1982, 1984a, 1984b, 1986; Solomon, 1976; Weiner, 1986).

Dans les revues comparatives de ces théories de l'évaluation et de la différenciation des émotions, Scherer (1988, 1999b) a tenté de montrer l'important degré de convergence des différentes suggestions théoriques, en particulier concernant les critères centraux postulés dans les différentes approches. Cette ressemblance apparaît comme des plus surprenantes puisque les théoriciens viennent de traditions de psychologie et/ou de philosophie largement différentes. Les empreintes laissées par les théories de l'évaluation et de la différenciation de l'émotion ont généré un corps de recherche qui s'est accumulé depuis lors, les confirmant également par des études empiriques comparatives (Lazarus, \& Smith, 1988; Manstead, \& Tetlock, 1989; Reisenzein, \& Hoffman, 1990; Roseman, Spindel, \& Jose, 1990; Scherer, 1988). L'état actuel de cette approche théorique et de la recherche empirique pertinente est présenté de manière exhaustive dans Scherer, Schorr, \& Johnstone (200I).

20 Nous illustrerons brièvement les principaux tenants des théories de l'évaluation et leurs prédictions, puisque cette approche est de plus en plus acceptée comme un modèle valide d'explication de la plupart des états émotionnels humains. Ce faisant, nous nous centrerons sur le modèle du Groupe de Recherche en Emotions de Genève, la théorie des processus composants de Scherer. Le Tableau I ci-dessous mentionne les critères d'évaluation, dénommés séquences de traitement de la stimulation («stimulus evaluation checks») dans le modèle développé par Scherer (et comparés à quelques autres auteurs). 
TABLEAU I - Critères d'évaluation des séquences de traitement de la stimulation dans le modèle de Scherer et comparaison de ceux-ci avec les critères d'autres théoriciens de l'appraisal

\begin{tabular}{|c|c|}
\hline $\begin{array}{l}\text { Séquence de traitement } \\
\text { de la stimulation (Scherer) }\end{array}$ & $\begin{array}{c}\text { Appraisal dimensions (Frijda, } \\
\text { Ortony/Clore, Roseman, Smith/Ellsworth, } \\
\text { Solomon,Weiner) }\end{array}$ \\
\hline Nouveaute & Change, attentional activity(?) \\
\hline Soudaineté & $\gamma$ \\
\hline Familiarité & Familiarity \\
\hline Prévisibilité & Unexpectedness \\
\hline Agrement intrinseque & Valence, appealingness, pleasantness \\
\hline \multicolumn{2}{|l|}{ Rapports aux buts } \\
\hline Pertinence & $\begin{array}{l}\text { Focality, appetitive/aversive motivation, } \\
\text { scope/focus, importance }\end{array}$ \\
\hline $\begin{array}{l}\text { Degré de certitude dans la prédiction } \\
\text { des conséquences }\end{array}$ & $\begin{array}{l}\text { Certainty, probabillity, likelihood, } \\
\text { predictability }\end{array}$ \\
\hline Attente & Presence, prospect realization \\
\hline Opportunité & $\begin{array}{l}\text { Open/closed, desirability, motive } \\
\text { consistency, goal/path obstacle, evaluation }\end{array}$ \\
\hline Urgence & Urgency, proximity \\
\hline \multicolumn{2}{|l|}{ Pontentiel de maitrise } \\
\hline Causalité: interne & $\begin{array}{l}\text { Intent/self-other, agency, responsability, } \\
\text { locus of causality }\end{array}$ \\
\hline Causalité: externe & Agency, stability \\
\hline Contrôle & Modifiability, controllability \\
\hline Puissance & Controllability, power \\
\hline \multicolumn{2}{|l|}{ Ajustement } \\
\hline \multicolumn{2}{|l|}{ Accord avec les standards } \\
\hline Standards externes & Value relevance, legitimacy, fairness \\
\hline Standards internes & Blameworthiness \\
\hline
\end{tabular}

Quant au Tableau 2, elle présente un exemple des prédictions qui peuvent être générées sur la base d'une théorie de l'évaluation de ce type. L'idée générale est que l'évaluation subjective d'une situation, d'un événement ou d'un stimulus, sur la base de ces critères d'évaluation, détermine la nature 
(c'est-à-dire à la fois la qualité et l'intensité) de la réaction émotionnelle (Tableau 2, exemples pour colère, peur et tristesse). II convient ici de préciser que ces critères n'ont pas à correspondre à la nature objective de la situation - en fait, l'écart entre évaluation subjective et nature objective de la situation figure souvent à la base de désordres affectifs, voir Scherer, 1987).

TABLEAU 2 - Exemples de prédictions générées sur la base du modèle des processus composants

\begin{tabular}{|c|c|c|c|}
\hline $\begin{array}{c}\text { Séquence de traitement } \\
\text { de la stimulation }\end{array}$ & Colère/Rage & Peur/Panique & Tristesse \\
\hline \multicolumn{4}{|l|}{ Nouveaute } \\
\hline Soudaineté & élevée & élevée & basse \\
\hline Familiarité & basse & ouvert & basse \\
\hline Prévisibilité & basse & basse & ouvert \\
\hline Agrement intrinseque & ouvert & & \\
\hline \multicolumn{4}{|l|}{ Rapports aux buts } \\
\hline Pertinence & ordre & corps & ouvert \\
\hline $\begin{array}{l}\text { Degré de certitude dans la prédiction } \\
\text { des conséquences }\end{array}$ & très élevé & élevé & très élevé \\
\hline Attente & dissonante & dissonante & ouvert \\
\hline Opportunité & enrayée & enrayée & enrayée \\
\hline Urgence & élevée & très élevée & basse \\
\hline \multicolumn{4}{|l|}{ Potentiel de maitrise } \\
\hline Causalité: interne & autrui & autrui/nature & ouvert \\
\hline Causalité: externe & intentionnelle & ouvert & $\begin{array}{l}\text { Chance } \\
\text { /négative }\end{array}$ \\
\hline Contrôle & élevé & ouvert & très bas \\
\hline Puissance & élevé & très basse & très basse \\
\hline Ajustement & élevé & bas & moyen \\
\hline \multicolumn{4}{|l|}{ Accord avec les standards } \\
\hline Standards externes & bas & ouvert & ouvert \\
\hline Standards internes & bas & ouvert & ouvert \\
\hline
\end{tabular}


La plupart des théories de l'appraisal n'ont pas tenté de prédire les processus micro-génétiques impliqués dans l'évaluation des antécédents de l'émotion. Une exception cependant pour ce modèle des processus composants qui postule que les critères d'évaluation proposés (stimulus evaluation checks) se produisent selon une séquence invariante (dans l'ordre indiqué dans les Tableaux I et 2). Cette notion de séquence, basée sur des considérations phylogénétiques, ontogénétiques et micro-génétiques (logiques), soutient l'idée que le processus d'évaluation est constamment opérant avec des évaluations continuellement réalisées pour mettre à jour l'information de l'organisme en fonction d'un événement ou une situation (incluant le besoin et la possibilité d'agir sur ceux-ci). Leur succession très rapide et leur opération continue peuvent expliquer les changements soudains qui se produisent durant les épisodes émotionnels et qui sont souvent basés sur des réévaluations de l'événement ou de nos propres capacités de faire face (cf. «secondary apparaisal» (Lazarus, 1968); voir Scherer 1984a, 1999a, 200 I, pour davantage de détails sur le traitement séquenciel dont il est fait l'hypothèse).

Les théories de l'appraisal sont souvent accusées d'un cognitivisme excessif, l'argument étant que la plupart des émotions semblent se développer beaucoup plus vite et beaucoup plus spontanément qu'elles ne le feraient si on supposait qu'elles étaient des traitements cognitifs approfondis. L'erreur de cette critique réside dans le fait que le traitement évaluatif de l'information est assimilé à un traitement cortical. Cette supposition est, bien sûr, injustifiable. Leventhal et Scherer (1987) ont montré qu'on peut envisager l'existence d'un traitement à plusieurs niveaux avec un organisme à même de s'adapter automatiquement au niveau qui est suffisant et efficace pour les besoins déterminants du moment. Le Tableau 3 montre comment ce traitement à plusieurs niveaux a été conçu par ces auteurs. Aujourd'hui, l'idée d'un traitement à niveaux multiples est fortement. répandu dans les domaines de la recherche sur les émotions, sur le traitement de l'information, et les troubles affectifs (voir Van Reekum \& Scherer, 1997, pour une synthèse).

Il est difficile d'accepter des fonctions ou mécanismes postulés théoriquement qui ne sont pas supportés, ou voire même contredits, par l'organisation d'un substrat.

Les développements théoriques sur la nature du processus d'évaluation et les tentatives éventuelles de modélisation en un paradigme de réseau 
TABLEAU 3 - Niveau de traitement des checks (SECs)

\begin{tabular}{|c|c|c|c|c|c|}
\hline & Nouveaute & $\begin{array}{l}\text { Agrement } \\
\text { intrinseque }\end{array}$ & $\begin{array}{l}\text { Rapports } \\
\text { aux buts }\end{array}$ & $\begin{array}{c}\text { Potentiel } \\
\text { de maitrise }\end{array}$ & $\begin{array}{l}\text { Accord } \\
\text { Avec les } \\
\text { standards }\end{array}$ \\
\hline $\begin{array}{c}\text { Niveau } \\
\text { conceptuel }\end{array}$ & $\begin{array}{c}\text { Attentes: } \\
\text { cause/effet/, } \\
\text { estimation des } \\
\text { probabilités }\end{array}$ & $\begin{array}{c}\text { Evaluation +/- } \\
\text { par anticipation, } \\
\text { souvenir ou } \\
\text { découlement }\end{array}$ & $\begin{array}{l}\text { Buts, plans } \\
\text { conscients }\end{array}$ & $\begin{array}{c}\text { Capacité à } \\
\text { résoudre des } \\
\text { problèmes }\end{array}$ & $\begin{array}{l}\text { Idéal de Soi } \\
\text { évaluation } \\
\text { morale }\end{array}$ \\
\hline $\begin{array}{c}\text { Niveau } \\
\text { schématique }\end{array}$ & $\begin{array}{l}\text { Familiarité: } \\
\text { comparaison } \\
\text { des schémas }\end{array}$ & $\begin{array}{c}\text { Préférences/ } \\
\text { aversions } \\
\text { apprises }\end{array}$ & $\begin{array}{c}\text { Besoins, } \\
\text { mobiles } \\
\text { acquis }\end{array}$ & $\begin{array}{l}\text { Schéma du } \\
\text { corps }\end{array}$ & $\begin{array}{l}\text { Schémas du } \\
\text { Soi et du social }\end{array}$ \\
\hline $\begin{array}{c}\text { Niveau } \\
\text { sensorimoteur }\end{array}$ & $\begin{array}{l}\text { Soudaineté: } \\
\text { stimulatión } \\
\text { intense }\end{array}$ & $\begin{array}{c}\text { Préférences/ } \\
\text { aversions innées }\end{array}$ & $\begin{array}{l}\text { Besoins de } \\
\text { base }\end{array}$ & $\begin{array}{c}\text { Energie } \\
\text { disponible }\end{array}$ & $\begin{array}{c}\text { (Adaptation } \\
\text { empathique?) }\end{array}$ \\
\hline
\end{tabular}

neuronal trouveront une avancée importante si l'on tient compte des développements récents sur la connaissance des structures anatomiques et des circuits dans le fonctionnement du SNC ayant rapport à l'émotion (Scherer, 1993b). Les travaux de LeDoux (1987, 1989) relatifs aux mécanismes du cerveau impliqués dans l'évaluation de la signification affective du traitement cognitif sont des plus pertinents en la matière. Ses minutieux travaux sur l'identification de circuits fonctionnels dans la perception et l'apprentissage émotionnel chez le rat ont fourni des preuves à l'existence d'une projection directe thalamico-amygdalienne. Les stimuli complexes sont

24 traités dans les noyaux sensoriels thalamiques et reliés aux régions néocorticales en vue d'une analyse et d'une synthèse perceptuelles détaillées. Cependant, des caractéristiques plus simples de ces stimuli activent des cellules thalamiques qui communiquent directement avec l'amygdale et peuvent fournir une évaluation rudimentaire de la signification affective de ces stimuli. Dans ce sens, l'amygdale est conçue comme satisfaisant à une fonction homéostatique générale, en évaluant la signification des informations reçues de sources extéroceptives et intéroceptives et en amorçant rapidement les réponses comportementales et viscérales correspondantes. 
Nous supposons que les deux premiers critères d'évaluation, nouveauté et caractère intrinsèque, du stimulus sont de ces caractéristiques «simples» qui devraient être traitées très rapidement parce que les circuits sensoriels parallèles ont accès à l'amygdale. Elles se produiraient tout à fait au début de la séquence théorique d'évaluation des checks proposée, parce que (a) elles se produisent plus tôt dans la séquence de traitement sensoriel, (b) elles utilisent des circuits plus courts, (c) les cellules thalamiques, dans lesquelles la projection vers l'amygdale se produit, ont des propriétés de règlage relativement faibles (voir LeDoux, 1987, p. 436).

Ces caractéristiques pourraient également expliquer l'argument de Zajonc (1980) pour défendre l'idée d'un système de traitement affectif primaire. La plupart des exemples de Zajonc, si ce n'est tous, impliquent des stimuli qui sont susceptibles d'englober ces aspects de nouveauté et d'agrément intrinsèque. L'évaluation de la signification affective des stimuli sensoriels via les neurones limbiques (par ex. pour la nouveauté ou le caractère agréable) est susceptible de se produire inconsciemment. Si nous en savions davantage sur les structures qui médiatisent le traitement des différentes dimensions d'évaluation, on pourrait alors avoir une meilleure mainmise sur le problème épineux de la conscience dans le traitement émotionnel - particulièrement, en ce qui concerne l'état affectif subjectif.

II convient de relever que la discussion précédente n'implique pas que les structures sub-corticales permettent une évaluation interprétative très approfondie ou sophistiquée. Bien entendu, seules des caractéristiques relativement simples du stimulus peuvent être traitées à ces niveaux. Comme Leventhal et Scherer (1987) ont tenté de le démontrer, la force du système d'évaluation humain réside dans le fait que des niveaux différents d'évaluation sont disponibles et que le traitement peut être adapté à la nature du stimulus ou des besoins interprétatifs de l'organisme. La suggestion d'un processus d'évaluation à plusieurs couches trouve support dans la démonstration de LeDoux (1989) attribuant à l'amygdale et l'hippocampe des fonctions différentiées dans l'évaluation de la signification affective du stimulus. La projection directe thalamico-amygdalienne permet un traitement grossier mais très rapide correspondant au niveau le plus bas, le niveau sensori-moteur du traitement selon le modèle de Leventhal \& Scherer (1987). L'hippocampe est beaucoup plus lent pour répondre aux inputs sensoriels, mais ceux-ci sont intégrés, au travers plusieurs modalités, à un traitement complexe du cortex associatif, avant d'atteindre l'hippocampe, 
ce qui permet, par la suite, un traitement beaucoup plus sophistiqué. Ce circuit pourrait correspondre au niveau schématique du modèle de Leventhal et Scherer (1987), puisque l'on peut supposer que le cortex associatif utilise les patterns en tant que schèmes dans son prétraitement des inputs (voir Figure 2, ci-dessous).

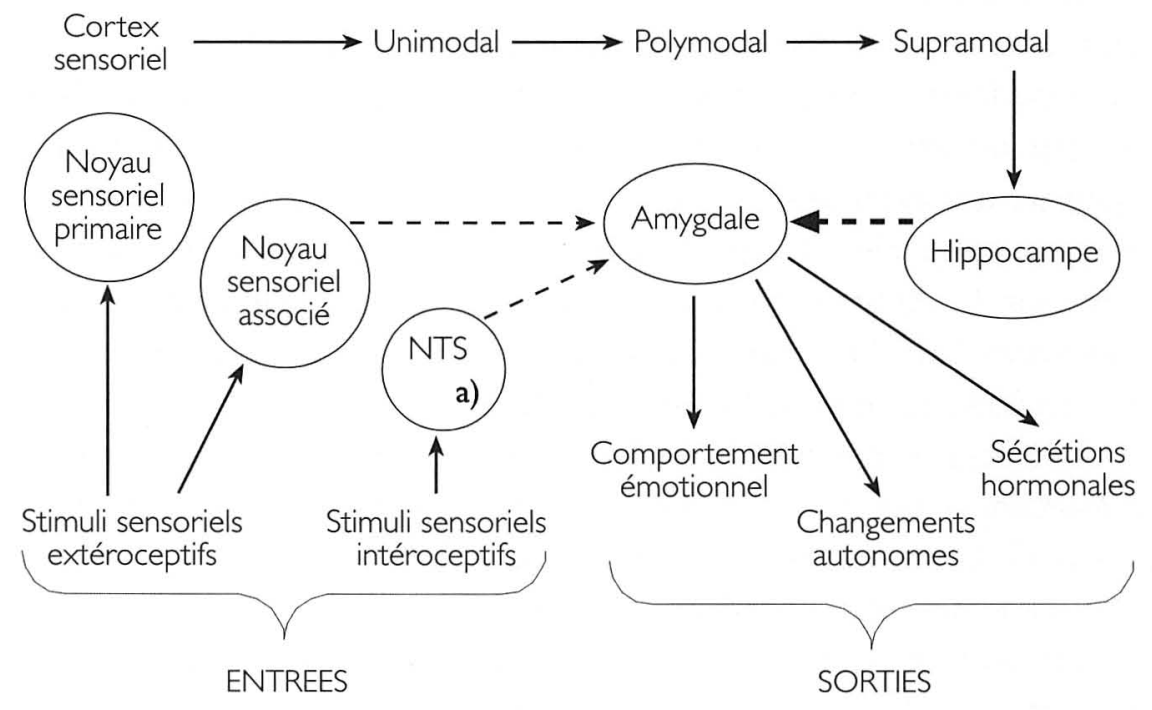

Figure 2 - Niveaux de traitement neuronal de stimuli sonores utilisés dans le conditionnement de la peur chez le rat

(traduit et adapté de LeDoux, 1987)

a) NTS = «nucleus of solitary tract» = noyau de l'appareil cellulaire

Cet argument de «cascades» séquentielles, ou à plusieurs niveaux, de l'évaluation du stimulus est soutenu par de récents travaux menés dans le laboratoire de LeDoux sur les connexions acoustiques de l'amygdale latérale chez le rat. Ces études ont montré que l'information sensorielle, retransmise par les noyaux médiaux et ventraux du corps géniculer médial, atteint directement l'amygdale latérale et à différents intervalles de temps, après un pré-traitement par différentes structures, incluant le cortex périrhinal (LeDoux, communication personnelle). 
Tout cela pour la théorie. Qu'en est-il des faits? II y a actuellement un corps impressionnant de recherches empiriques pour démontrer la validité de ces suggestions hypothétiques (Ellsworth \& Smith, 1988; Frijda, 1987; Frijda, Kuipers, \& ter Schure, 1989; Gehm \& Scherer, 1987; Manstead \& Tetlock, 1989; Reisenzein \& Hoffman, 1990; Roseman, 1984; 1991; Roseman, Spindel, \& José, 1990; Smith \& Ellsworth, 1985, 1987; Tesser, 1990; Weiner, 1986, ainsi que Scherer, 1999b pour un survol). Nous fournirons deux brèves illustrations tirées du programme de recherche de notre laboratoire de Genève.

Dans le cadre d'une étude inter-culturelle à grande échelle (Scherer \& Wallbott, 1994), 2921 répondants de 37 pays ont dû se rappeler d'expériences récentes de joie, tristesse, peur, colère, dégoût, honte et culpabilité et répondre à des questions concernant leur évaluation du comportement ou événement ayant suscité l'émotion. Les résultats obtenus soutiennent la plupart des prédictions du modèle des processus composants, mais pas toutes. Les analyses discriminantes suggèrent qu'un nombre relativement petit de dimensions d'évaluation pourrait être suffisant pour classifier les émotions principales avec un degré de précision appréciable (voir Tableau 4).

TABLEAU 4 - Matrice des résultats résultants des analyses discriminantes

\begin{tabular}{c|c|r|r|r|r|r|r|r}
\hline \multirow{2}{*}{$\begin{array}{c}\text { Emotion } \\
\text { réelle }\end{array}$} & \multirow{2}{*}{$\begin{array}{c}\text { Nbre } \\
\text { de cas }\end{array}$} & \multicolumn{7}{|c}{ Emotion prédite } \\
\cline { 3 - 9 } & Joie & Peur & Colère & Tristesse & Dégoût & Honte & Culpabilité \\
\hline Joie & 530 & 98.3 & .9 & .0 & .2 & .0 & .4 & .2 \\
Peur & 352 & 4.0 & 16.5 & 19.6 & 21.3 & 10.8 & 14.2 & 13.6 \\
Colère & 600 & 1.3 & 8.8 & 47.0 & 17.7 & 12.3 & 7.3 & 5.5 \\
Tristesse & 365 & 3.0 & 8.5 & 19.2 & 36.7 & 9.0 & 13.7 & 9.9 \\
Dégoût & 449 & 2.0 & 13.8 & 33.2 & 12.5 & 23.8 & 7.3 & 7.3 \\
Honte & 546 & 5.1 & 9.0 & 16.1 & 11.0 & 7.0 & 26.4 & 25.5 \\
Culpabilité & 542 & 3.5 & 5.9 & 15.3 & 9.4 & 6.1 & 23.1 & 36.7 \\
\hline
\end{tabular}


Les comparaisons inter-culturelles ont montré que l'effet principal de l'émotion explique davantage de variance que l'effet principal du pays ou l'interaction émotion $X$ pays. Ceci insinue que, bien que le mécanisme d'évaluation et les critères principaux semblent être quasi universels, il y a des différences culturelles dans l'ampleur d'implication des critères d'évaluation spécifiques pour la différenciation des émotions.

L'analyse des facteurs culturels qui peuvent expliquer ces différences démontre l'importance des systèmes de valeurs, tels l'individualisme ou le collectivisme, et des croyances culturelles. Par exemple, les participants Africains ont attribué un degré d'immoralité à événements émotionnels beaucoup plus fort que les participants d'autres parties du monde. II semble que cette tendance soit liée aux croyances au surnaturel dans les pays Africains (Scherer, 1997).

Le Groupe de Recherche en Emotions de Genève a commencé à explorer les techniques développées par la tradition de l'intelligence artificielle pour examiner les manières par lesquelles l'évaluation des antécédents de l'émotion peut aller au-delà du paradigme établi consistant à établir des preuves corrélationnelles entre les rapports verbaux d'expériences émotionnelles verbalement étiquetées et les dimensions d'évaluation inférées. Dans une première étude, Scherer (1993b) a montré qu'une approche «système expert» peut être utilisée pour examiner combien et quels types spécifiques de dimensions d'évaluation sont requises pour diagnostiquer de façon satisfaisante les états émotionnels rapportés par les utilisateurs de ce système. Les données ont montré que leur précision dépasse largement ce qui pourrait être imputé à la chance (voir Figure 3). L'approche «système expert» mène à des suggestions très précises qui ont permis des reformulations et modifications des assomptions théoriques, de même qu'à des nécessités de changements des hypothèses, au vu de preuves

28 empiriques contradictoires. En conséquence, cette approche pourrait fournir un principe de comparaison des théories rivales dans ce domaine et apporter davantage de convergence sur ce sujet.

En collaboration avec Pascal Edwards, Susanne Kaiser et Thomas Wehrle, nous sommes en train d'essayer d'améliorer et d'élargir les algorithmes de l'ordinateur et de développer des outils de simulation pour le développement d'une théorie et l'analyse exploratoire de données. Le prototype d'un tel outil a été élaboré: le GATE - Geneva Appraisal Theory Environment - qui permet, par l'utilisation d'extension graphique, l'évaluation interactive des 


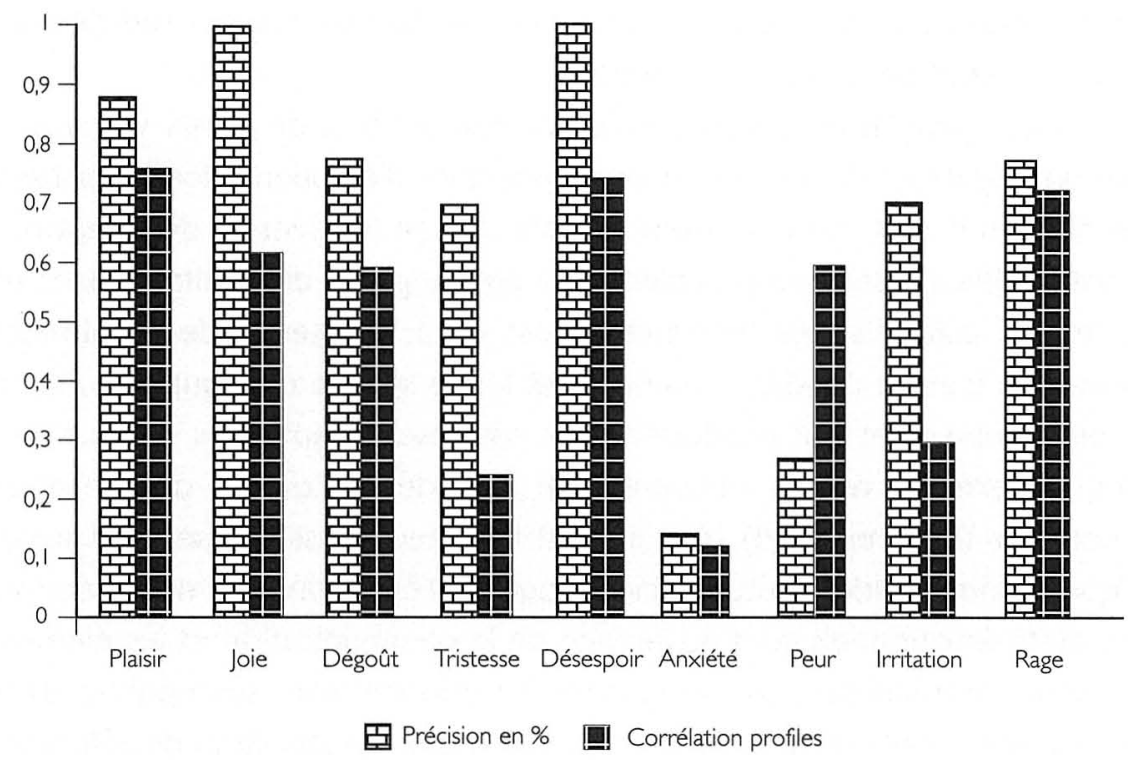

Figure 3 - Précision diagnostique du système expert GENESE, basé sur Scherer, 1993b. (Précision en \% = Pourcentage des prédictions correctes du système expert; Corrélation profiles $=$ Pearson $r$ entre les profils moyens empiriques obtenus et les profils théoriquement prédits sur la base de 15 checks).

effets de changements dans les prédictions théoriques. De façon générale, nous pensons qu'il est utile d'aller au-delà d'une méthodologie classique et d'employer de nouveaux paradigmes de recherches en incluant des simulations par ordinateur et des modèles d'agents autonomes (Wehrle \& Scherer, 2001).

\section{Les émotions constituent des états d'un système organisé de façon} complexe plutôt que des programmes de réponses préfigurées

Une controverse maintenant classique en psychologie des émotions concerne le nombre et le type d'émotions de base caractérisées par des patterns de réponses typiques (voir le chapitre spécial Cognition et Emotion, Vol. 6, 1992). Nous soutiendrons que beaucoup des arguments produits dans 
cette controverse sont égarants et que nous devons changer radicalement notre conception d' «une émotion».

Un des problèmes les plus insidieux est l'emploi de labels verbaux en tant qu'indicateurs du nombre et de l'importance d'émotions «fondamentales». Le fait qu'il soit relativement simple de se référer à des réponses émotionnelles assez comparables, dans des langages différents et dans des contextes culturels très hétéroclites, est dû à l'existence de nombreuses similarités dans la condition humaine et la vie sociale qui sont évaluées de façon identique et qui produisent des réponses adaptatives comparables. Ces patterns universels peuvent être considérés comme des émotions «modales» (Scherer, 1994). Mais il serait trompeur d'user d'une terminologie laïque comme guide à l'étude scientifique de l'émotion. Les mots décrivant des états émotionnels sont au service de la communication et les éléments qui sont communiqués sur un épisode émotionnel sont susceptibles d'être fort variables, car pouvant s'étendre à toute une constellation de références aux antécédents de la situation, allant du type d'évaluation, de l'état physiologique jusqu'aux caractéristiques complexes du sentiment. Malheureusement, la communication scientifique, tout comme notre langage quotidien, dépend pour ses références de moyens verbaux et nous avons dès lors de sérieux problèmes à nous affranchir de ce corset.

II convient entre parenthèses de souligner qu'il serait également trompeur de considérer le fait que les étiquettes verbales et les états affectifs peuvent être appréhendés facilement au travers un petit nombre de dimensions, telles la valence ou l'activité qui ont été amplement employés depuis Wundt (1903; voir Osgood et al., 1975) comme base au développement des théories de l'émotion (par ex. Russell, 1980). Une fois encore, les dimensions du sentiment et sa sémantique sont des images, plutôt que les discriminants de l'émotion. Ils représentent des dispositifs descriptifs qui sont intéressants en eux-même mais qui ne nous fournissent pas d'éclaircissements sur la nature de l'émotion.

Nous croyons également qu'il est déroutant de définir les émotions de base essentiellement à partir de leur configuration des expressions faciales (Ekman, 1984, 1992; Izard, 1971, 1977; Tomkins, 1984). Bien qu'il y ait de bonnes preuves que des acteurs laïques ou professionnels peuvent produire de façon précise des expressions faciales pouvant être reconnues même au travers différentes cultures, ceci n'apporte pas la preuve que l'expression faciale de l'émotion est en effet hautement standardisée et limitée à quelques patterns prototypiques. Quelques travaux récents, en dépit du fait qu'il y ait 
recours à des acteurs qui pourraient être considérés comme s'appuyant sur des expressions hautement schématiques, trouvent un degré assez important de variabilité dans l'expression faciale affichée (Galati, Scherer, \& Ricci-Bitti, 1997).

En suivant ces mises en matière, démarrées par Duchenne et Darwin, puis poursuivies par les contributions de Sancte de Sanctis (1904) et de la psychologie de l'expression allemande (Lersch, 1932/197I; Schänzle, 1939), on peut poser l'argument que l'expression faciale est componentielle, avec des unités musculaires individuelles reflétant soit l'évaluation, soit les tendances à l'action - au service d'une fonction adaptative (Frijda, \& Tcherkassof, 1997; Scherer, 1992; Smith, \& Scott, 1997). La configuration de l'expression faciale dans une telle perspective est le résultat cumulatif de mouvements «en pièces détachées» qui, étant donné la large variabilité des patterns d'évaluation et des combinaisons de tendances à l'action, sont susceptibles d'être relativement variables au travers les individus et les situations.

Des arguments semblables peuvent être avancés pour l'expression vocale de l'émotion. Dans une étude récente, Banse et Scherer (1996) ont montré qu'il y a des profils de caractéristiques acoustiques pour différentes émotions, mais que ceux-ci pouvaient être assez dissemblables en fonction de membres différents des familles d'émotion (telles que une colère chaude vs. froide, anxiété vs. panique). De plus, il ressort passablement de variabilité en fonction des acteurs. Par ailleurs, les différences dans la précision avec laquelle les profils vocaux de l'émotion sont reconnus par les juges semblent liées aux aspects fonctionnels des vocalisations de l'émotion (par ex. le dégoût est plutôt faiblement reconnu et la cause présumée est que nous sommes en fait peu susceptibles de maintenir des settings fonctionnels du dégoût dans nos régions oro-pharyngiales et faciales qui se produisent lors de la rencontre de quelque chose de déplaisant).

Comme pour le visage, on peut défendre l'argument que l'expression vocale reflète l'évaluation et les tendances à l'action, plutôt que des expressions de catégorie d'émotion pure. En partant de données récentes sur les fonctions représentatives et symboliques des cris chez les primates (Marler, 1984), Scherer (1986) a soulevé la question de savoir si la vocalisation affective humaine pourrait être également au service de fonctions cognitives représentatives aussi bien que de fonctions émotionnelles-motivationnelles. Un modèle développé antérieurement sur les fonctions de signal, le modèle «Organon» proposé par Bühler (1933, 1934), qui postule des fonctions de signalement, de symptômes et de 
symboles pour tous les signaux, permet de conceptualiser la nature multi-fonctionnelle de l'expression vocale. De plus, une distinction peut être faite entre des facteurs «push» qui «poussent»l'expression affective, principalement via les effets physiologiques, et les facteurs «pull» qui «tirent» leur expression via les modèles socialement médiatisés. Les facteurs «pull» semblent souvent être impliqués dans les fonctions de signalement. Bien que les facteurs «push», aux bases physiologiques, fournissent souvent des symptômes sur l'état de l'émetteur, on peut avancer qu'ils peuvent également servir à des fonctions cognitives représentatives dans le sens qu'ils reflètent les processus cognitifs d'évaluation des antécédents qui ont produit l'état affectif chez l'émetteur (voir Scherer, 1986, pour les détails).

L'étude psycho-physiologique des réponses émotionnelles, où s'est révélée la difficulté de démontrer empiriquement des configurations stables des paramètres physiologiques qui seraient typiques pour certaines émotions, pourrait être aussi résolue par une approche componentielle. De nouveau, en revenant sur la valeur fonctionnelle de la réponse physiologique (par ex. Lacey et al, 1963), il se justifie d'affirmer que les changements physiologiques pourraient être dirigés de manière componentielle: en fournissant l'adaptation physiologique nécessaire au traitement de l'information ou aux tendances à l'action produits par l'évaluation des antécédents de l'émotion (voir Stemmler, 1984).

L'approche componentielle décrite ci-dessus nécessite un nouveau paradigme pour étudier les dépendances et inter-relations du système, étant donné que la notion classique de «programmes expressifs neuro-moteurs» (Ekman, 1972, 1984; Tomkins, 1963) ne semble pas très adéquate pour rendre compte des mécanismes impliqués. Une approche prometteuse serait d'utiliser des approches dynamiques non linéaires (par exemple la théorie du chaos ou de la catastrophe) pour modéliser les caractéristiques de la réponse émotionnelle. La nature transitoire de l'épisode émotionnel, caractérisé par des changements d'état et la synchronisation de tous les sous-systèmes organismiques, peut être comparée aux modèles physiques des oscillateurs couplés, l'état du système étant temporairement contrôlé par des attracteurs étrangers (Redington, \& Reidbord, 1992). De même, les changements abrupts dans l'état émotionnel et les effets de l'état initial, peuvent être joliment modélisés par la théorie de la catastrophe (Zeeman, 1976). Dans ce sens, Scherer (2000) a suggéré d'adopter une approche dynamique et non linéaire à la modélisation de l'émotion. 
En conclusion, en étudiant l'émotion en tant que mode d'opération de l'organisme, dans le sens d'une synchronisation de plusieurs composantes d'un «système opérant», plutôt qu'en tant que système relativement séparé ou séries de programmes neurophysiologiques rigides, on ouvre la voie à un grand nombre de perspectives prometteuses pour les théories et recherches futures, qui convergeraient avec certains changements dans les paradigmes fondamentaux, observables dans la plupart des disciplines actuelles, en direction de complexité et de non-déterminisme.

\section{REFERENCES}

Arnold, M.B. (1960). Emotion and personality. (Vol. I) Psychological aspects. New York: Columbia University Press.

Banse, R., \& Scherer, K.R. (1996). Vocal profiles of affect: Evidence for 14 emotions. Journal of Personality and Social Psychology, 70(3), 614-636.

Bergson, H. (1940). Le rire. Essai sur la signification du comique. Paris : Presses Universitaires de France: Quadrige.

Bühler, K. (1933). Ausdruckstheorie. Jena: Fischer.

Bühler, K. (1934). Sprachtheorie. Jena: Fischer.

Clapier-Valadon, S. (1991). L'homme et le rire. Encyclopédie La Pléiade, Histoire des Moeurs, Vol. III. Paris : Editions Gallimard.

De Rivera, J. (1977). A structural theory of the emotions. Psychological Issues, 10 (4), 178.

Dumas, G. (1948a). La vie affective. Paris: Presses Universitaires de France.

Dumas, G. (1948b). Le Sourire, Psychologie et Physiologie. Paris : Presses Universitaires de France.

Dumas, G. (1900). La Tristesse et la Joie. Paris : Félix Lacan Editeur.

Ekman, P. (1972). Universals and cultural differences in facial expression of emotion. In J.R. Cole (Ed.), Nebraska Symposium on Motivation (pp. 207- 283). Lincoln: University of Nebraska Press.

Ekman, P. (1984). Expression and the nature of emotion. In K.R. Scherer \& P. Ekman (Eds.), Approaches to emotion (pp. 319-344). Hillsdale, N.J.: Erlbaum.

Ekman, P. (1992). An argument for basic emotions. Cognition and Emotion, 6, 169-200.

Ellsworth, P.C., \& Smith, C.A. (1988). From appraisal to emotion: Diffferences among unpleasant feelings. Motivation and Emotion, 12, 27I-302.

Fraisse P. (1969). Motivation, Emotion et Personnalité. In Traité de Psychologie Expérimentale, Vol IX, Presses Universitaires de France, Paris.

Frijda, N. (1986). The Emotions. Cambridge \& New York : Cambridge University Press.

Frijda, N. (1987). Emotion. Cognitive structure and action readiness. Cognition and Emotion, I, $115-143$.

Frijda, N. H., \& Tcherkassof, A. (1997). Facial expressions as modes of action readiness. In Russell, J. A., \& Fernández-Dols, J. M. (Eds.), The psychology of facial expression (pp. 78- 102). Cambridge: Cambridge University Press.

Frijda, N., Kuipers P., \& Ter Schure E. (1989). Relations Among Emotion, Appraisal, and Emotional Action Readiness. Journal of Personality and Social Psychology, 57(2), 212-228. 
Galati, D., Scherer, K. R., \& Ricci-Bitti, P. E. (1997). Voluntary facial expression of emotion: Comparing congenitally blind with normally sighted encoders. Journal of Personality and Social Psychology, 73(6), | 363-1379.

Gehm, Th., \& Scherer, K. R. (1987). Emotionsantezedente Faktoren als subjektive Dimensionierungskriterien emotionsbeschreibender Adjektive. Sprache und Kognition, 2 , $51-63$.

Izard, C.E. (197I). The face of emotion. New York: Appleton- Century-Crofts.

Izard, C.E. (1977). Human emotions. New York: Plenum.

Janet, P. (1926). La pensée intérieure et ses troubles. Compte rendu intégral du cours professé par P. Janet au Collège de France. Psychologie expérimentale et comparée. Paris : Publications A. Chahine.

Johnson-Laird, P.N. \&, Oatley, K. (1989). The Language of emotions: An analysis of a semantic field. Cognition and Emotion, 3, 81-123.

Kaiser, S., \& Scherer, K. R. (1998). Models of 'normal' emotions applied to vocal and facial expression in clinical disorders. In W. F. Flack, Jr. \& J. D. Laird (Eds.), Emotions in Psychopathology, (pp. 81-98). New York: Oxford University Press.

Lacey, J.I, Kagan, J., Lacey, B.C., \& Moss, H.A. (1963). The visceral level: Situational determinants and behavioral correlates of autonomic response patterns. In P.H. Knapp (Ed.), Expression of the emotions in man. New York: International Universities Press, 161-196.

Lazarus, R.S. (1968). Emotions and adaptation: Conceptual and empirical relations. In W.J. Arnold (Ed.). Nebraska Symposium on Motivation (Vol. 16, pp. 175-270). Lincoln, Ne.: University of Nebraska Press.

Lazarus, R.S., \& Smith, C.A. (1988). Knowledge and appraisal in the cognition-emotion relationship. Cognition and Emotion, 2, 281-300.

LeDoux, J.E. (1987). Emotion. In F. Plum \& V. Mountcastle (Eds.), Handbook of physiology. Nervous system, Vol. 5. Higher Functions (pp. 419-459). Washington, DC: American Physiological Society.

LeDoux, J.E. (1989). Cognitive-emotional interactions in the brain. Cognition and Emotion, 3 , 267-289.

Lersch, P. (1932/197|). Gesicht und Seele: Grundlinien einer mimischen Diagnostik. München: Reinhart. Leventhal, H., \& Scherer, K.R. (1987). The relationship of emotion and cognition: A functional approach to a semantic controversy. Cognition and Emotion, I, pp. 3-28.

Lorenz, K. (1965). Ueber tierisches und menschliches Verhalten. Aus dem Werdegang der Verhaltenslehre. München: Pieper.

Malrieu, Ph. (1952). Les émotions et la personnalité de l'enfant. Etudes de Psychologie et de Philosophie, XII. Paris : Librairie Philosophique J.Vrin.

Manstead, A.S., \& Tetlock, P.E. (1989). Cognitive appraisals and emotional experience: further evidence. Cognition and Emotion, 3, 225-240.

Marler, P. (1984). Animal communication: Affect of Cognition? In K.R. Scherer \& P. Ekman (Eds.), Approaches to emotion (pp. 345-368). Hillsddale, N.J.: Erlbaum.

Mees, U. (1985). Was meinen wir, wenn wir von Gefühlen reden? Zur psychologischen Textur von Emotionswörtern. Sprache und Kognition, 1, 2-20.

Ortony, A., Clore, G., \& Collins, A. (1988). The cognitive structure of emotions. Cambridge : Cambridge University Press.

Osgood, C.E., May, W.H., \& Miron, M.S. (1975). Crosscultural universals of affective meaning, Urbana, III, University of Illinois Press. 
Pradines, M. (1954). Sur les conceptions actuelles de l'émotion. La Psychologie du XXème siècle. Paris : Presses Universitaires de France.

Redington, D. J., \& Reidbord, S. P. (1992). Chaotic Dynamics in Autonomic Nervous System Activity of a Patient During a Psychotherapy Session. Biological Psychiatry, 31, 993- 1007.

Reisenzein, R., \& Hoffmann, T. (1990). An investigation of dimensions of cognitive appraisal in emotion using the repertory grid technique. Motivation and Emotion, 14, I-26.

Roseman, I.J. ( 1984). Cognitive determinants of emotion: A structural theory. In P. Shaver (Ed.) Review of personality and social psychology, (Vol. 5, pp. II-36). Beverly Hills, CA: Sage.

Roseman, I.J. (1991). Appraisal determinants of discrete emotions. Cognition and Emotion, 5, |6|-200.

Roseman, I.J., Spindel, M.S., \& Jose, P. E. (1990). Appraisal of emotion-eliciting events: Testing a theory of discrete emotions. Journal of Personality and Social Psychology, 59, 899-9।5.

Russel, J.A. (1980). A circumplex model of affect. Journal of Personality and Social Psychology, $39,161-178$.

Sanctis, S. de (1904). La Mimica del Pensiero. Milano: Sandron

Schänzle, J. (1939). Der mimische Ausdruck des Denkens. Berlin: Bernard \& Graefe.

Scherer, K.R. (198I). Wider die Vernachlässigung der Emotion in der Psychologie. In W. Michaelis (Ed.) Bericht über den 32. Kongress der Deutschen Gesellschaft für Psychologie in Zürich 1980, (pp. 304-317). Göttingen: Hogrefe.

Scherer, K.R. (1982). Methods of research on vocal communication: Paradigms and parameters. In K.R. Scherer \& P.Ekman (Eds.). Handbook of methods in nonverbal behaviour research (pp.136-198). Cambridge: Cambridge University Press.

Scherer, K.R. (1984a). On the nature and function of emotion: A component process approach. In K.R. Scherer, \& P. Ekman (Eds.). Approaches to emotion (pp. 293-328). Hillsdale, N.J.: Erlbaum.

Scherer, K.R. (1984b). Emotion as a multicomponent process: A model and some cross-cultural data. In P. Shaver, \& L. Wheeler (Eds.). Review of Personality and Social Psychology (Vol. 5, pp. 37-63). Beverly Hills, CA: Sage.

Scherer, K. R. (1984c). Les Emotions : Fonctions et composantes. Cahiers de Psychologie Cognitive, 1, 9-39 (repris dans Rimé, B., \& Scherer, K. R. (Eds) (1989). Les Emotions. Neuchâtel-Paris : Delachaux \& Niestlé.

Scherer, K.R. (1986). Vocal affect expression: A review and a model for future research. Psychological Bulletin, 99, 143-165.

Scherer, K.R. (1987). Vocal assessment of affective disorders. In J.D. Maser, (Eds.) Depression and expressive behavior (pp. 57-82). Hillsdale, NJ: Erlbaum.

Scherer, K.R. (1988). Criteria for emotion-antecedent appraisal: A review. In V. Hamilton, G.H. Bower \& N. H. Frijda (Hrsg.), Cognitive perspectives on emotion and motivation. (pp. 89126). Dordrecht: Kluwer.

Scherer K.R. (1993a). Neuroscience Projections to Current Debates in Emotion Psychology. Cognition and Emotion, 7(I), I-4I.

Scherer K.R. (1993b). Studying the Emotion-Antecedent Appraisal Process: An Expert System Approach. Cognition and Emotion, 7 (3/4), 325-355.

Scherer, K.R., \& Wallbott, H.G. (1994) Evidence for Universality and cultural Variation of Differential Emotion Response Patterning. Journal of Personality and Social Psychology, 66(2), 310-328.

Scherer, K. R. (1997). The role of culture in emotion-antecedent appraisal. Journal of Personality and Social Psychology, 73, 902-922. 
Scherer, K. R. (1999a). On the sequential nature of appraisal processes : Indirect evidence from a recognition task. Cognition and Emotion, I3(6), 763-793.

Scherer, K. R. (1999b). Appraisal theories. In T. Dalgleish, \& M. Power (Eds.), Handbook of Cognition and Emotion (pp. 638-663). Chichester: Wiley.

Scherer. K. R. (2000). Emotions as episodes of subsystem synchronization driven by nonlianear appraisal processes. In M. D. Lewis \& I. Garnic (Eds.), Emotion, development, and selforganization : Dynamic systems approaches to emotional development (pp. 70-99). Cambridge \& New York : Cambridge University Press.

Scherer, K. R. (200I). Appraisal considered as a process of multi-level sequential checking. In K. R. Scherer, A. Schorr., \& T. Johnstone (Eds.), Appraisal processes in emotion : Theory, Methods, Research (pp. 92-120). New York and Oxford : Oxford University Press.

Scherer, K. R., Schorr, A., \& Johnstone, T. (Eds.) (200I). Appraisal processes in emotion : Theory, Methods, Research. New York and Oxford: Oxford University Press.

Smith, C.A., \& Ellsworth, P.C. (1985). Patterns of cognitive appraisal in emotion. Journal of Personality and Social Psychology, 48, 8I3-838.

Smith, C.A., \& Ellsworth, P.C. (1987). Patterns of appraisal and emotion related to taking an exam. Journal of Personality and Social Psychology, 52, 475-488.

Smith, C. A., \& Scott, H. S. (1997). A componential approach to the meaning of facial expressions. In J. A. Russell \& J. M. Fernandez-Dols (Eds.), The psychology of facial expression (pp. 229-254). Cambridge \& New York: Cambridge University Press.

Solomon, R.C. (1976). The passions. The myth and nature of human emotion. Garden City, NY: Doubleday.

Stemmler, G. (1984). Psychophysiologische Emotionsmuster (Psychophysiological patterning of emotion). Frankfurt, Germany: P. Lang

Tesser, A. (1990). Smith and Ellsworth's appraisal model of emotion: A replication, extension, and test. Personality and Social Psychology Bulletin, 16, 210-223.

Tomkins, S.S. (1984). Affect Theory. In K.R. Scherer \& P. Ekman (Eds). Approaches to emotion (pp. 63-196). Hillsdale. NJ: Lawrence Erlbaum Associates Inc.

Van Brakel, J. (1994). Emotions: a cross-cultural perspective on forms of life. Social Perspectives on Emotion, 2, 179-237.

Van Reekum, C. M., \& Scherer, K. R. (1997). Levels of processing for emotion-antecedent appraisal. In G. Matthews (Ed.), Cognitive Science Perspectives on Personality and Emotion (pp. 259-300). Amsterdam : Elsevier Science.

Wallon, H. (1938). La vie mentale. Paris : Editions sociales.

Wallon, H. (1934). Les origines du caractère chez l'enfant. Paris : Boivin \& Cie Eds.

Wehrle T., \& Scherer K.R. (200I). Towards computational modeling of appraisal theories. In K. R. Scherer, A. Schorr, \& T. Johnstone (Eds.). Appraisal processes in emotion :Theory, Methods, Research (pp. 350-365). New York: Oxford University Press.

Weiner, B. (1986). An attributional theory of motivation and emotion. New York: Springer.

Wundt, W. (1903). Grundzüge der physiologischen Psychologie, Band 3. Leipzig, Engelmann.

Zajonc, R. B. (1980). Thinking and feeling: Preferences need no inferences. American Psychologist, $35,151-175$.

Zeeman, E. C. (1976). Catastrophe Theory. Scientific American, 234 (4), 65-83. 


\section{Pierre Philippot}

Unité de recherche en Psychologie Clinique: Emotion, Cognition, Santé

Université de Louvain à Louvain-la-Neuve, Belgique

\section{FACTEURS COGNITIFS ET RÉACTIONS CORPORELLES DANS LE PROCESSUS ÉMOTIONNEL}

Les relations entre changements corporels et sentiments émotionnels sont au centre d'un débat qui n'a cessé d'animer la psychologie scientifique depuis sa fondation (James, 1890; Personality and Social Psychology Bulletin, 1990). Ce débat aborde, dans le domaine spécifique que constituent les émotions, la question fondamentale du rapport entre les processus biologiques et les processus psychologiques. II est donc également traversé par les grands courants de la philosophie occidentale qui a toujours prescrit un dualisme entre le monde physique (le corps) et la pensée.

Dans le chapitre présent, cette question va être présentée sous l'angle de la psychophysiologie sociale. Nous allons examiner, d'une part, les relations entre les changements physiologiques objectifs et l'expérience émotionnelle subjective - les sentiments - et, d'autre part, l'expérience des sensations corporelles et le sens qui leur est attribué par le sujet de l'émotion. Plus spécifiquement, la première partie du chapitre est consacrée aux développements historiques des théories modélisant changements corporels et sentiments subjectifs. Les données empiriques relatives à la différenciation physiologique des émotions seront passées en revue et critiquées. Ensuite, nous examinerons les données empiriques relatives aux sensations corporelles dont les individus font l'expérience durant leurs expériences émotionnelles. Enfin, nous présenterons une série d'expériences effectuées dans notre laboratoire et consacrées à la modélisation des processus qui génèrent ces sensations et à leur fonction dans le processus émotionnel. Développement historique des théories articulant changements corporels et expériences émotionnelles

D'un point de vue historique, on peut considérer que les théories contemporaines des relations entre sensations corporelles et émotion ont 
été précédées par une évolution en trois étapes majeures, chaque étape étant marquée par une théorie: la théorie périphérique de James, la théorie thalamique de Cannon et la théorie socio-cognitive de Schachter.

\section{William James: la théorie périphérique}

La question des changements physiologiques et des sensations corporelles fut pour la première fois mise au centre des théories de l'émotion par William James (1884). Le point central de la contribution de James est l'inversion de la séquence théorique du processus émotionnel. En effet, jusqu'alors on avait toujours considéré qu'un stimulus émotionnel engendre un état émotionnel marqué par un sentiment subjectif chez l'individu qui perçoit ce stimulus et que cet état donne lieu à des réactions corporelles. Au contraire, James propose que, par un processus associatif, le stimulus émotionnel engendre une réponse corporelle et que le sentiment émotionnel ne soit rien d'autre que l'aperception de l'état corporel suscité par le stimulus émotionnel.

II est important de souligner la position de James sur la différenciation physiologique des émotions. Pour lui, comme il n'y a jamais deux états corporels semblables, il ne peut y avoir deux sentiments émotionnels semblables. James ne postule donc pas du tout des patterns physiologiques typiques pour chaque émotion, comme le fera Lange (1884) et plus tard les théoriciens des émotions de bases, comme Ekman (1984). Pour illustrer sa position, James utilise la métaphore d'un instrument de musique, tel une guitare: les organes seraient les cordes et on peut y produire une infinité d'accords (les combinaisons d'activations différentes des organes) qui sont amplifiés dans la caisse de résonance que constitue le corps. Dans cette métaphore, le son produit serait le sentiment subjectif. Tout comme certains accords produisent une impression semblable, différents «Do» par exemple, il y aurait au niveau corporel des états relativement semblables les uns aux autres. Ces similitudes d'états corporels produiraient des similitudes au niveau des sentiments émotionnels. Ainsi, bien que nous ne soyons jamais deux fois dans le même état corporel lorsque nous sommes en colère, la similitude entre tous ces états serait suffisante pour que nous puissions chaque fois leur donner la même étiquette, celle de colère.

La position de James est donc particulièrement nuancée et contemporaine en ce qu'elle tient compte de la multitude d'états corporels possibles. De 
plus, elle propose une approche prototypique de la catégorisation émotionnelle, des états semblables étant regrouper sous un même prototype. Enfin, du point de vue cognitif qui nous intéresse plus particulièrement ici, dans la théorie de James, les changements corporels sont l'unique déterminant du sentiment émotionnel de l'individu.

\section{Walter Cannon: la théorie thalamique}

La théorie de James fut vigoureusement attaquée par Walter Cannon $(1915,1931)$ qui formula un ensemble de critiques (Cannon, 1927). Ces critiques peuvent se résumer en 3 points essentiels: (a) les viscères sont des organes relativement insensibles; (b) les changements viscéraux sont beaucoup plus lents que les changements de sentiments subjectifs et (c) les changements viscéraux sont indifférenciés et non-spécifiques aux émotions. Cependant, ces arguments ne sont pas tous vraiment pertinents contre la théorie de James et ils ont été contestés (Fehr \& Stern, 1970; Wegner, 1960). En résumé, les contre-arguments sont les suivants. Premièrement, les arguments de Cannon ne prennent en compte que les viscères, alors que James considérait l'ensemble des réactions corporelles, y compris les changements au niveau de la musculature squeletale. Or, ceux-ci sont beaucoup plus rapides que les changements viscéraux, très finement innervés - et donc très sensibles - et, comme l'ont démontré les travaux sur l'expressivité faciale, hautement différenciés (Ekman, 1984).

Bien que contestables, les arguments de Cannon eurent un grand impact sur la psychologie des émotions, de même que la théorie qu'il formula (Cannon, 1915, 1929). Dans cette théorie, dite thalamique, Cannon propose que les différentes facettes de l'émotion, tant les facettes subjectives que les facettes physiologiques, sont organisées au niveau du système nerveux central. Alors que James pensait, à tort, que les récepteurs sensoriels étaient directement projetés au niveau du cortex sensoriel, Cannon note qu'ils sont tous interrompus au niveau thalamique. Selon Cannon (1929), le thalamus serait le centre d'organisation des émotions. Ses impulsions auraient deux effets. D'une part, au niveau périphérique, elles commanderaient l'activation des changements physiologiques, d'autre part, au niveau cortical, elles induiraient le sentiment subjectif de l'émotion. Selon la théorie de Cannon, le thalamus serait spontanément en perpétuel état d'excitation. Cette 
excitation serait contenue, inhibée par le cortex. Cependant, la propriété spécifique des stimuli émotionnels serait de lever cette inhibition, soit par action directe dans le cas des stimuli inconditionnels, soit après un relais cortical dans le cas des stimuli conditionnels.

D'un point de vue psychologique, la théorie de Cannon est basée sur un processus d'apprentissage classique très rudimentaire. Cannon était avant tout un physiologiste, sa théorie est donc peu développée au niveau psychologique. Mais les deux idées de base de la théorie de Cannon, à savoir l'indifférenciation physiologique et la détermination au niveau du SNC du sentiment émotionnel, furent reprises et développées d'un point de vue psychologique dans la théorie de Schachter (1964).

\section{Stanley Schachter: la théorie socio-cognitive}

Schachter part des mêmes prémices que Cannon: l'activation physiologique émotionnelle est indifférenciée et la distinction des sentiments subjectifs dépend d'un processus central. Pour Schachter, il s'agit d'un processus cognitif d'évaluation de la situation émotionnelle et principalement de ses composantes sociales.

Selon Schachter, la séquence du processus émotionnel est la suivante: un stimulus déclenche une activation physiologique. Celle-ci est indifférenciée et le processus reliant le stimulus à l'activation physiologique n'est pas spécifié par Schachter (Mandler [1984] fera une proposition théorique à ce sujet). Cette activation donne lieu à des sensations corporelles qui sont perçues par l'individu. Celui-ci aurait une motivation innée à donner une explication à son état d'activation (Festinger, 1954), et en l'absence d'autres indices, se tournerait vers son environnement pour donner sens à ses sensations. $\mathrm{Si}$ la signification est d'ordre émotionnel, l'individu ressentira une émotion. Selon Schachter, il y a donc trois conditions nécessaires pour qu'il y ait émotion (Reizensein, 1983): présence d'une activation physiologique, perception des sensations corporelles qui en découlent, et interprétation de cet état d'activation en termes émotionnels.

On le voit, si les prémices des théories thalamique et schachterienne sont les mêmes, la séquences des processus est différente. Mais en plus, dans la théorie schachterienne, les changements corporels jouent un rôle très important: ils sont une condition nécessaire à l'émotion. Condition 
néanmoins non suffisante, en ce que l'activation physiologique doit être interprétée comme ayant une cause émotionnelle pour qu'il y ait sentiment subjectif émotionnel.

Du point de vue cognitif, les sensations corporelles sont une source d'information importante pour l'individu: elle lui signale la mobilisation de son organisme consécutivement à une stimulation vraisemblablement émotionnelle. Cette source d'information est cependant incomplète car indifférenciée. Elle doit être complétée par de l'information provenant du contexte, et - dans la vision de Schachter fortement influencé par son maître Festinger - principalement du contexte social.

\section{Les théories historiques: conclusion}

Les trois théories qui viennent d'être présentées - périphérique, thalamique, et socio-cognitive - présentent des vues assez divergentes sur le processus émotionnel et sur les relations entre changement corporel et sentiment subjectif. Les divergences concernent principalement le caractère différencié ou non des changements physiologiques et les relations causales qu'entretiennent ou non les sensations corporelles et le sentiment subjectif. Ces divergences non résolues constituent le terreau dans lequel se sont développées les théories contemporaines.

\section{LES THÉORIES CONTEMPORAINES}

Les théories contemporaines pourraient être grossièrement classées en deux grandes catégories: les théories biologiques et les théories cognitives. Les premières se sont développées dès la fin des années 70' et sont marquées par une inspiration néo-darwinienne. Les secondes sont apparues un peu plus tardivement dans la foulée du développement des sciences cognitives.

\section{Les théories biologiques: Izard et Ekman}

Izard (1969, 1977) et Ekman (1973, 1984) ont proposé relativement indépendamment deux théories très semblables que nous qualifierons de 
théories biologiques à cause de leur fort ancrage darwinien. Celles-ci postulent une organisation biologique innée de l'émotion. L'émotion constituerait un ensemble de processus relativement automatiques d'adaptation à l'environnement. Ces processus comprendraient un ensemble de réponses pré-organisées aux niveaux viscéral, musculaire, expressif et subjectif. Pour Izard (1977) comme pour Ekman (1984), les différentes émotions sont donc clairement caractérisées par des patterns physiologiques et expressifs différents.

La séquence émotionnelle procéderait d'une manière relativement simple. Un stimulus émotionnel déclencherait de manière innée ou par association (conditionnement classique) l'activation d'un programme émotionnel inné. De l'activation de ce programme résulteraient les différentes réponses émotionnelles. Cependant, l'activation d'une facette de cette réponse, par rétroaction provoquerait l'activation du programme central, et par là, l'activation des autres facettes. Ainsi, adopter une expression faciale ou une posture émotionnelle résulterait en l'activation du programme émotionnel et du sentiment subjectif correspondant. La recherche empirique a en effet démontré de manière très consistante, que l'activation d'une réponse corporelle de type émotionnel engendrait l'état subjectif correspondant, pour la modulation les expressions faciales (Matsumoto, 1987), de la posture (Stepper \& Strack, 1993) et de la respiration (Philippot, Chapelle \& Blairy, 2002).

Les théories d'lzard et d'Ekman diffèrent quant à l'origine du sentiment subjectif. Pour lzard, le sentiment émotionnel provient directement et essentiellement d'une rétroaction périphérique, comme chez James. Cependant, pour Ekman, le sentiment émotionnel est avant tout déterminé par le programme central qui organise l'ensemble des réponses émotionnelles, y compris, dans ce cas, la facette subjective. Quoi qu'il en soit, dans les deux théories, les changements corporels influent sur le sentiment subjectif, soit de manière directe (Izard), soit via l'activation du programme émotionnel central (Ekman).

\section{Les théories cognitives: Scherer et Frijda}

Le point de vue biologique a été largement modulé par les théories cognitives, telles celles de Scherer (1984) ou de Frijda (1986). Une des 
grandes contributions de ces auteurs est d'avoir développé au niveau théorique le processus d'évaluation de la situation. Dans les trois modèles historiques présentés en début de chapitre, il est très évident que cet aspect du processus n'avait reçu aucun développement théorique. De même, à cet égard, la contribution des théories biologiques s'est limitée à des notions de stimuli inconditionnels et conditionnés.

Les théories de l'évaluation ont été largement présentées dans d'autres chapitres du présent volume. Elles ne vont donc pas être pleinement développées ici. Du point de vue de la thématique du présent chapitre, celui des relations entre cognition et changements corporels, ces théories offrent une perspective particulièrement intéressante. En effet, tant Scherer que Frijda ont proposé que les changements corporels dépendent de souscomposants cognitifs du processus émotionnel.

Par exemple, dans le cadre de la théorie componentielle des émotions, Scherer (1991) a proposé que le résultat de chaque séquence d'évaluation du stimulus entraîne une modification physiologique spécifique. Par exemple, une évaluation positive à l'étape de nouveauté entraînerait les changements cardio-vasculaires propres à la surprise: un ralentissement du rythme cardiaque suivi d'une accélération. Les différentes étapes de la séquence d'évaluation donneraient ainsi lieu à différents types de réactions corporelles. L'ensemble de ces réactions résulterait en un profil physiologique qui serait spécifique au sentiment émotionnel de l'individu, étant donné que ce sentiment serait déterminé par les mêmes facteurs que ceux régissant les réactions corporelles.

Un autre concept particulièrement prometteur pour la compréhension des changements corporels dans les émotions est celui de tendance à l'action, formulé par Nico Frijda (1986). Dans sa théorie, Frijda conçoit l'émotion comme une forme de réponse aux demandes d'une situation. L'émotion consiste en des processus d'analyse de la situation - l'évaluation - et en des processus de programmation de l'action: les tendances à l'action. L'émotion prépare donc l'individu à accomplir certaines actions préférentiellement à d'autres. Dans ce contexte, il est tout-à-fait fonctionnel que cette préparation à l'action inclue aussi l'activation des systèmes biologiques nécessaires à la bonne exécution de l'action en préparation. Les changements physiologiques de l'émotion seraient donc le soutien logistique de la tendance à l'action. 


\section{Les théories contemporaines: conclusions}

Dans la perspective des deux théories cognitives, les changements physiologiques sont déterminés par des processus spécifiques du phénomène émotionnel et non par un programme émotionnel central comme dans les théories biologiques. Les processus proposés sont les séquences d'évaluation de la situation pour Scherer et les tendances à l'action pour Frijda. Alors que cette perspective met l'accent sur la modélisation de processus sous-tendant les phénomènes émotionnels, la perspective des théories biologiques est centrée sur la notion de programmes innés et sur le concept d'émotion de base qui y est attaché

En résumé, nous pourrions synthétiser les questions théoriques relatives à la physiologie des émotions en trois points: (a) les changements physiologiques sont-ils une condition nécessaire à l'émotion, (b) les différentes émotions sont-elles caractérisées par un pattern spécifique de changements physiologiques, et (c) les changements physiologiques interviennent-ils dans les sentiments subjectifs qui accompagnent les émotions? Malheureusement, il n'y a d'accord théorique sur aucune des trois questions. Alors que James, Schachter et les théories biologiques contemporaines considèrent que les changements physiologiques sont une condition nécessaire à l'émotion, les autres pensent que ces changements participent aux processus mais ne sont en rien nécessaires. Les divergences sont les plus importantes quant à la différenciation physiologique des émotions, les uns, tel James, prônant une différenciation absolue, les autres, tels Cannon ou Schachter, défendant l'idée d'une non différenciation tout aussi absolue. Enfin, il existe de nombreuses propositions théoriques concernant les liens entre changements corporels et sentiment subjectif, depuis l'absence de lien (Cannon), au lien causal direct et univoque (James),

44 en passant par la modulation de l'intensité du sentiment émotionnel (Schachter). Face à cette diversité théorique, quel est l'apport de la recherche empirique?

\section{RECHERCHES EMPIRIQUES SUR LA PHYSIOLOGIE DES ÉMOTIONS}

L'abondance de la littérature théorique sur ce sujet contraste avec la pauvreté de la recherche empirique. Si cette recherche a une longue 
tradition, les premières études contrôlées remontant à Ax (1953), peu d'études ont été spécifiquement consacrées à la physiologie des émotions, vraisemblablement à cause des difficultés méthodologiques d'induction d'émotions en laboratoire et de mesure et d'analyse des réponses physiologiques. Les procédures les plus utilisées pour induire les émotions ont été l'imagerie mentale (Schwartz, Weinberger \& Singer, 1981), la présentation d'extraits de films (Averill, 1969) ou de diapositives à signification émotionnelle (Lang, Bradley, \& Cuthbret, 1990). Malheureusement, les procédures de recherche n'ont jamais été standardisées, tant pour la mesure des changements physiologiques que pour l'induction des émotions en laboratoire (Pour une critique méthodologique des études psychophysiologiques des émotions, voir Stemmler, 1992.). De plus, pratiquement toutes ces recherches ont été conçues pour contraster des émotions dites «de base» à d'autres ou à un état neutre. Pratiquement aucune recherche n'a examiné si des processus émotionnels spécifiques, tels les tendances à l'action ou les séquences d'évaluation de la situation émotionnelle, avaient une signature physiologique propre.

Malgré la disparité de la littérature à ce sujet, Stemmler et Meinhardt (1990) ont tenté une revue de la question. De celle-ci, il ressort qu'il n'y a actuellement pas de preuve empirique de l'existence de patterns physiologiques différenciant les émotions de base. Les résultats mis en évidence par une étude ou une procédure de recherche sont souvent contredits par d'autres études, utilisant ou non des procédures différentes. Le seul résultat qui semble stable est que la colère est généralement marquée par une tension artérielle systolique plus importante que n'importe quelle autre émotion.

II faut cependant interpréter avec prudence les résultats d'une telle méta-analyse. En effet, les auteurs insistent très justement sur le fait que la question de la différenciation physiologique des émotions n'a pas encore été abordée par des études suffisamment solides au plan méthodologique pour pouvoir répondre validement à cette question. Idéalement, différentes émotions devraient être induites dans différents contextes et par différentes procédures, et un ensemble suffisamment large de mesures physiologiques devraient être enregistré. Etant donné la difficulté méthodologique, ne fût-ce que d'induire en laboratoire une émotion écologiquement valide (Philippot, 1993), l'étude idéalement contrôlée de la physiologie des émotions n'est sans doute pas prête de voir le jour. 


\section{SENSATIONS CORPORELLES ET ÉMOTION}

Malgré l'intérêt théorique de la question, il n'y a donc pas actuellement de preuve empirique de l'existence de patterns physiologiques différenciant les émotions de base. II existe cependant une autre source de données empiriques pour aborder le problème de la différenciation physiologique des émotions, à savoir les sensations corporelles que les individus ressentent lors d'expériences émotionnelles.

\section{Les sensations corporelles rapportées après des émotions}

Dans quelques recherches (Pennebacker, 1982; Scherer, Wallbott, \& Summerfield, 1986; Shields, 1984; Rimé \& Giovanni, 1986), les participants ont été invités à se remémorer des expériences émotionnelles personnelles et ont dû se rappeler les sensations corporelles qu'ils ont ressenties durant chacune de ces expériences. Une recension de ces recherches a donné lieu à des résultats très surprenants (Philippot, 1992; Philippot \& Rimé, 1997). Chaque émotion de base y est caractérisée par un pattern spécifique de sensations corporelles. Ces patterns sont remarquablement semblables entre les différentes études, bien que celles-ci aient été effectuées avec des méthodologies très différentes. Ainsi, la joie est marquée par des sensations d'accélération cardiaque et de détente musculaire, la colère, par des sensations de chaleur, de transpiration, de tensions musculaires et d'accélération cardiaque et respiratoire, alors que la peur évoque une sensation de froid, de boule dans la gorge, d'accélérations cardiaque et respiratoire, et de tremblements musculaires.

Nous nous trouvons donc ici devant un paradoxe: Comment est-il possible que les individus puissent rapporter de manière aussi consistante des patterns de sensations corporelles spécifiques à chaque émotion alors que les études psychophysiologiques, utilisant des mesures très sensibles, n'ont pu mettre en évidence de tels patterns? Ce constat est d'autant plus étonnant que les recherches sur la viscéroception ont montré que les individus peuvent généralement percevoir leurs changement physiologiques mieux que le niveau prédit par le hasard (Pennebacker, 1982). Ce paradoxe constitue le point de départ d'un programme d'investigations empiriques développé dans notre laboratoire. 


\section{Hypothèses de recherches}

Trois hypothèses permettant de rendre compte de ce paradoxe ont été envisagées. La première hypothèse, qualifiée de sociale-constructiviste, pose qu'il existe des représentations culturelles attribuant à chaque émotion des changements physiologiques typiques. Etant donné leur origine culturelle, ces représentions n'entretiendraient pas de liens causaux avec les changements physiologiques réels. Selon l'hypothèse sociale-constructiviste, les sensations corporelles perçues ou remémorées par les sujets ne seraient que le reflet de ces représentations culturelles.

La seconde hypothèse, qualifiée de biologique, est diamétralement opposée à l'hypothèse sociale-constructiviste. Dans cette seconde optique, les sensations corporelles seraient déterminées par les changements physiologiques réels. L'absence de pattern stable dans les études psychophysiologique de l'émotion serait due au fait que, dans ces études, étant donné les conditions de laboratoire, les émotions n'auraient pas été induites de manière suffisamment intense pour susciter des changements physiologiques marqués.

Enfin, la troisième hypothèse, qualifiée de prototypique, propose une solution médiane. II existerait des représentations spécifiant les changements physiologiques typiques de chaque émotion. Cependant, en contraste avec l'hypothèse sociale-constructiviste ces représentations ne seraient pas d'origine socioculturelle. Elles seraient constituées sur la base d'expériences émotionnelles significatives, ayant engendré des changements physiologiques suffisamment intenses que pour être perçus. II s'agirait en quelque sorte d'exemplaires.

La mise à l'épreuve de ces trois hypothèses exige l'examen des relations causales entre trois types de variables. Le premier type est constitué par les changements physiologiques, c'est-à-dire les réponses physiologiques qui peuvent être objectivement mesurées lors d'une émotion. Le second type concerne les sensations corporelles, qui désignent les symptômes et sensations physiques ressentis et perçus par le sujet lors d'une expérience émotionnelle. Enfin, les schèmes psychophysiologiques (Cacioppo, Andersen, Turnquist, \& Tassinary, 1989) désignent les représentations spécifiant les sensations corporelles typiques de chaque émotion. 
Les schèmes psychophysiologiques et leur rapport avec les sensations corporelles

La question la plus immédiate à laquelle il fallait répondre concerne l'existence des schèmes psychophysiologiques. En effet, l'existence en mémoire de représentations abstraites qui spécifient les sensations corporelles typiques pour chaque émotion est une condition nécessaire aux hypothèses prototypique et sociale-constructiviste. Pour répondre à cette question, nous avons mené l'expérience suivante (Philippot, 1992). Les participants ont reçu pour simple instruction de mentionner les sensations corporelles que la plupart des gens ressentent lors d'une émotion donnée. Ils durent exécuter cette tâche à un rythme très rapide: les participants ne disposaient que d'une seconde pour évaluer l'intensité typique de chaque sensation corporelle. Cette procédure avait pour but de forcer les participants à baser leurs réponses sur des représentations abstraites, c'est à dire sur leur mémoire sémantique, et d'éviter qu'ils basent leurs réponses sur des souvenirs d'expériences émotionnelles qu'ils auraient réellement vécues, c'est-à-dire sur leur mémoire épisodique. L'efficacité de cette procédure à été vérifiée par un questionnaire post-expérimental.

Les résultats de cette étude sont très clairs: chaque émotion est caractérisée par un pattern type de sensations corporelles. On peut donc répondre affirmativement à la première question: il existe des schèmes psychophysiologiques spécifiant les sensations corporelles typiques de chaque émotion.

Cette réponse soulève immédiatement une autre question: les schèmes psychophysiologiques ainsi mis en évidence peuvent-ils rendre compte des sensations corporelles rapportées par les études mentionnées précédemment dans la revue de la littérature?

Pour répondre à cette question, nous avons comparé les patterns de sensations corporelles issus des schèmes psychophysiologiques avec ceux que les individus rapportent avoir ressentis lors d'un épisode émotionnel (Rimé, Philippot, \& Cisamolo, 1990). L'expérience comprenait donc deux conditions. Dans la première, la condition schématique, les participants devaient rapporter très rapidement, comme dans l'expérience précédente, les sensations corporelles typiques de quatre émotions (la joie, la colère, la peur et la tristesse). Dans la deuxième condition, qualifiée d'épisodique, les participants devaient se souvenir avec moult détails d'une expérience de joie, colère, peur ou tristesse qu'ils avaient récemment vécue. Ensuite, 
ils devaient indiquer l'intensité des sensations corporelles qu'ils avaient ressenties pendant ces émotions.

A nouveau, les résultats de cette étude sont très clairs: les patterns des sensations corporelles remémorées et des schèmes psychophysiologiques différencient clairement les quatre émotions étudiées. Mais surtout, en réponse à notre question, ces deux types de patterns sont parfaitement identiques, comme l'ont confirmé les analyses statistiques par MANOVA.

En considérant ces résultats, on pourrait se demander si l'équivalence ainsi observée entre schèmes psychophysiologiques et sensations corporelles remémorées post-hoc pourrait aussi être observée entre schèmes psychophysiologiques et les sensations corporelles perçues in situ, au moment même où l'individu fait l'expérience d'une émotion. En effet, les commentaires suivants pourraient être faits à propos de l'expérience qui vient d'être décrite. La mémoire des individus pour leurs sensations corporelles pourrait être défaillante, au moins partiellement. Dans ce cas, des processus de reconstruction mnémonique seraient automatiquement, et sans doute non consciemment, utilisés par les individus (Cacioppo et al., 1989; Ross, 1989). Ces processus reconstructifs seraient basés sur les schèmes psychophysiologiques. Ainsi, une tâche de rappel des sensations corporelles rend plus probable l'utilisation des schèmes psychophysiologiques qu'une tâche de perception in situ lors d'une expérience émotionnelle.

Pour examiner les relations existant entre schèmes psychophysiologiques et sensations corporelles perçues in situ, l'expérience suivante a été réalisée (Rimé et al., 1990). Dans une première condition, qualifiée de «perception in situ», les participants furent invités individuellement au laboratoire. Là, des extraits de films sélectionnés pour leur impact émotionnel (Philippot, 1993) leurs ont été montrés. Ces films ont induit des émotions de joie, colère, peur, tristesse, et dégoût. Chaque émotion a été induite par deux extraits de films différents. Juste à la fin de chaque extrait de film, ce qui correspond au moment d'intensité maximale de l'émotion, les participants décrivaient leurs sensations corporelles dans un questionnaire. Dans la deuxième condition, qualifiée de schématique, d'autres participants ont reçu une description des extraits de film. Ensuite, ils durent indiquer les sensations corporelles typiques qu'un individu moyen ressentirait à la vision de l'extrait de film.

Les résultats de cette expérience indiquent que les patterns des sensations corporelles perçus in situ et des schèmes psychophysiologiques 
différencient clairement les cinq émotions étudiées. Mais surtout, ces deux types de patterns sont très similaires. Des analyses statistiques plus poussées, utilisant les fonctions discriminantes, ont établi que les patterns des schèmes psychophysiologiques permettent d'identifier bien au-delà du niveau de chance les patterns perçus in situ.

En résumé, les expériences qui viennent d'être présentées indiquent qu'il existe une grande similitude entre les patterns de sensations corporelles remémorées post hoc ou perçues in situ et les patterns des schèmes psychophysiologiques. II se pourrait donc, comme le prédit l'hypothèse sociale-constructiviste, que les sensations corporelles soit déterminées par les schèmes psychophysiologiques. Cependant, la nature corrélative des données présentes, ne permet pas d'exclure l'hypothèse alternative, selon laquelle sensations corporelles et schèmes psychophysiologiques sont semblables parce que tous deux ont la même origine, à savoir les changements physiologiques objectifs.

\section{Sensations corporelles, changements physiologiques et schèmes psychophysiologiques}

Nous avons examiné les relations existantes entre sensations corporelles perçues in situ et changements physiologiques objectifs dans l'expérience suivante (Philippot, 1992). A nouveau, les participants furent invités individuellement au laboratoire où ils ont été exposés à des extraits de films émotionnels. Pendant la vision des films, une série de paramètres physiologiques ont été mesurés en continu. Après chaque extrait, les participants étaient invités à décrire leurs sensations corporelles dans un questionnaire. Ainsi, nous disposions pour chaque participant et chaque émotion des mesures de leurs sensations corporelles et des changements physiologiques correspondants.

Les résultats de cette expérience montrent que, comme dans l'expérience précédente, les participants rapportent avoir ressenti des sensations corporelles qui sont clairement différenciées d'après le type d'émotion induite. Par ailleurs, l'analyse des changements physiologiques mesurés objectivement indique que ceux-ci permettent aussi de différencier les émotions induites. Ainsi, d'une part, les participants rapportent des patterns de sensations corporelles différenciés selon le type d'émotion, et 
d'autre part, les instruments de mesures physiologiques indiquent que ces émotions ont donné lieu à des patterns d'activation différenciés.

Quelles relations entretiennent les changements physiologiques, les sensations corporelles et les schèmes psychophysiologiques? Si on crée des variables d'intensité générale pour ces trois domaines en additionnant les variables à l'intérieur de chacun des domaines, on remarque des corrélations de Spearman de l'ordre de .50, .60 indiquant que l'intensité ressentie ou attribuée des sensations corporelles n'est pas étrangère à l'activation globale de l'organisme.

Cependant, la question cruciale est de savoir si ces différences émotionnelles dans les patterns concordent au niveau qualitatif entre les sensations corporelles perçues et les changements physiologiques mesurés objectivement. Les analyses effectuées sur ces données indiquent que ces différences ne concordent pas. Des analyses corrélationnelles et des régressions multiples ont montré que la part de variance de sensations corporelles qui peut être expliquée par les changements physiologiques objectifs est très faible, au mieux de l'ordre de $7 \%$ de la variance si on entre tous les paramètres physiologiques comme prédicteurs dans l'équation. Ces résultats indiquent que, dans les conditions expérimentales que nous venons de décrire, les sensations corporelles rapportées par les participants ne peuvent être expliquées par les paramètres physiologiques correspondants.

En résumé, les résultats des expériences qui viennent d'être présentées montrent que (a) il existe des représentations abstraites de la physiologie des émotions: les schèmes psychophysiologiques, (b) les sensations corporelles remémorées post-hoc peuvent être prédites sur base des ces schèmes psychophysiologiques, (c) les sensations corporelles perçues in situ peuvent également être prédites sur base des ces schèmes psychophysiologiques, et (d) les sensations corporelles perçues ne semblent pas correspondre aux changements physiologiques mesurés objectivement in situ. Cet ensemble d'observations est très favorable à l'hypothèse sociale-constructiviste. Cependant, un élément central de cette hypothèse est que les schèmes psychophysiologiques sont des constructions culturelles. Une preuve empirique supplémentaire et déterminante en faveur de cette hypothèse serait donc de démontrer qu'il existe des différences culturelles dans les schèmes psychophysiologiques, et donc dans les sensations corporelles perçues lors d'émotions. Nous avons testé cette hypothèse dans deux études inter-culturelles. 


\section{Approche inter-culturelle des sensations corporelles et des schèmes psychophysiologiques}

Une première étude a abordé quatre pays: la Belgique, la Bolivie, I'Italie et les USA (Philippot, Rimé, Maric, Salderelli, \& Feldman, 1990). Dans cette étude, des étudiants universitaires ont dû décrire les sensations corporelles typiques de différentes émotions (joie, colère, peur et tristesse). Dans la deuxième étude, trois pays ont été abordés, la Belgique, l'Indonésie et le Mexique (Philippot, 1993b). En Indonésie, des étudiants de Jakarta et des individus appartenant à une ethnie reculée (Ranu Pani) vivant dans la jungle ont rapporté les sensations corporelles typiques de différentes émotions (joie, colère, peur, tristesse, dégoût, honte). Au Mexique, des étudiants et des indiens de la haute Sierra (Raramuri), vivant sans contact avec l'homme blanc, même via la radio, ont participé à l'étude. En Belgique, seuls des étudiants universitaires ont pris part à ce projet.

Ces deux études ont donné lieu à des résultats identiques: les similitudes entre cultures sont beaucoup plus importantes que leurs différences. En effet, alors que les différences culturelles n'expliquent que $5 \%$ de la variance des patterns de sensations corporelles rapportées par les participants, les aspects des patterns communs à toutes les cultures expliquent eux $32 \%$ de la variance. Ces résultats suggèrent donc qu'il ne semble pas exister de différences culturelles importantes dans les schèmes psychophysiologiques. Ils infirment donc l'hypothèse sociale-constructiviste et constituent un nouveau paradoxe: comment expliquer les similitudes inter-culturelles des schèmes psychophysiologiques alors que les recherches décrites auparavant suggèrent que ces schèmes psychophysiologiques ne peuvent pas être expliqués par les changements physiologiques objectifs?

\section{Les sensations corporelles: conclusions}

Les données actuelles de la littérature ne permettent pas d'exclure la possibilité que dans certaines circonstances, les individus soient capables de percevoir correctement leurs changements physiologiques. Cependant, prises dans leur ensemble, elles suggèrent que les processus dirigés par théories jouent un rôle important dans la perception et la remémoration des sensations corporelles. Je propose que dans la plupart des expériences 
émotionnelles, les schèmes psychophysiologiques jouent un rôle important dans la perception et le rappel des sensations corporelles. Ainsi, les sensations corporelles rapportées par les individus seraient, au moins en partie, le fruit d'un processus de construction et d'interprétation de la réalité physiologique de leur état. Ces processus de construction seraient basés sur les schèmes psychophysiologiques.

D'où proviennent alors ces schèmes psychophysiologiques? Les recherches menées dans notre laboratoire ne permettent pas de donner une réponse univoque à cette question. Je propose, à titre d'hypothèse de travail, que les schèmes psychophysiologiques peuvent avoir, non pas une, mais plusieurs origines. Nos résultats et la littérature suggèrent deux sources principales pour la constitution des schèmes psychophysiologiques.

La première est d'ordre sémantique. En effet, le simple fait d'une proximité sémantique entre concept d'émotion et concept de sensation corporelle pourrait expliquer que certaines sensations corporelles soient plus fréquemment associées à certaines émotions qu'à d'autres. Par exemple, le fait que le concept de chaleur ait une connotation positive et de légère activation pourrait expliquer que la sensation de chaleur soit associée à la joie, qui partage les même connotations sémantiques. A un niveau plus général, les métaphores et les théories populaires de l'émotion peuvent concourir à la formation des schèmes psychophysiologiques (Averill, 1974; Lakoff \& Kovesces, 1983).

La deuxième origine possible pour les schèmes psychophysiologiques est constituée par les expériences d'activation physiologique intense. Lors de telles expériences, les individus seraient capables de percevoir, au moins partiellement, leurs changements physiologiques réels. Si ces expériences sont de nature émotionnelle, les changements physiologiques perçus pourraient directement déterminer la constitution des schèmes psychophysiologiques.

\section{CONCLUSION GÉNÉRALE}

A priori, les changements viscéraux et les cognitions semblent être deux domaines bien différents et indépendants. Dans le présent chapitre, nous espérons avoir démontré que, si on prend le point de vue des émotions, il n'en est rien: l'interaction de ces deux domaines est nécessaire pour que naisse la facette phénoménale de l'émotion, les sentiments subjectifs. En 
amont, l'évaluation émotionnelle d'une situation par des processus cognitifs implicites et explicites est nécessaire au déclenchement des réponses physiologiques de l'émotion. En aval, l'interprétation des sensations corporelles diffuses détermine en partie l'intensité et la qualité du sentiment subjectif qui accompagne l'émotion. Comme l'avait déjà très élégamment souligné James (1884), que resterait-il comme sentiment d'une émotion dont on aurait effacé toute sensation corporelle?

L'exposé qui a précédé démontre que nous ne sommes qu'au début de la compréhension de ces interactions complexes entre réponses corporelles, cognitions et sentiments. De nouveaux modèles théoriques apparaissent (Philippot \& Schaefer, 200l) et tentent de mieux rendre compte de ces différents niveaux d'analyse. A ce titre, la nouvelle génération des théories componentielles et multi-niveaux initiées par Leventhal et Scherer (1987) est particulièrement prometteuse. De même, les développements récents des investigations de la physiologie, que ce soit aux niveaux viscéral ou cortical, ouvrent des perspectives fascinantes.

Les émotions offrent donc un domaine de recherche particulièrement privilégié, au carrefour des processus physiologiques et cognitifs, interaction dont découle toute la richesse phénoménologique de notre monde affectif et du sentiment-même que nous avons de nous-mêmes, et - in fine - de notre identité (Damasio, 1994) comme être de chair et d'esprit. Les émotions constituent à ce titre une des synthèses réintégrant la vieille et artificielle dichotomie occidentale du corps et de l'esprit. N'en déplaise à Descartes: « cogito, ergo movereo, ergo sum » : je pense, donc je m'émeus, donc je suis.

\section{RÉFÉRENCES BIBLIOGRAPHIQUES}

Averill, J. R. (1969). Autonomic response patterns during sadness and mirth. Psychophysiology, 5, 399-4I4.

Averill, J. R. (1974). An analysis of psychophysiological symbolism and its influence on theories of emotion. Journal for the Theory of Social Behavior, 4, 147-190.

Ax, A. F. (1953). The physiological differenciation between fear and anger in humans. Psychosomatic Medicine, 15, 433-442.

Cacioppo, J.T., Andersen, B.L., Turnquist, D.C., \& Tassinary, L.G. (1989). Psychophysiological comparison theory: On the experience description, and assessment of signs and symptoms. Patient Education and Counseling, 13, 257-270.

Cannon, W. B. (19/5). Bodily changes in pain, hunger, fear, and rage. (Ist ed.). New York: Appleton-Century. 
Cannon, W. B. (1929). Bodily changes in pain, hunger, fear, and rage. (2sd ed.). New York: Appleton-Century.

Cannon, W.B. (1927). The James-Lange theory of emotions: A critical examination and an alternative theory. American Journal of Psychology, 39, 106-124.

Cannon, W.B. (1931). Again the James-Lange and the thalamic theories of emotion. Psychological Review, 38, 281-295.

Ekman, P. (1973). Cross-cultural studies of facial expression. In P. Ekman (Ed.), Darwin and facial expression. New York: Academic Press.

Ekman, P. (1984). Expression and the nature of emotion. In K. Scherer and P. Ekman (Eds.) Approaches to emotion. Hillsdale, N.J.: Erlbaum.

Fehr, F.S. \& Stern, J.A. (1970). Peripheral physiological variables and emotion:The James-Lange theory revisited. Psychological Bulletin, 74, 41।-424.

Festinger, L. (1954). A theory of social comparison processes. Human Relations, 7, I I7- I 40.

Frijda, N.H. (1986). The emotions. Cambridge: Cambridge University Press.

Izard, C. E. (1977). Human emotions. New York: Plenum Press.

Izard, C.E. (1969). The emotions and emotion constructs in personality and culture research In R.B. Cattell (Ed.), Handbook of modern personality theory. Chicago: Adeline.

James, W. (1884). What is an emotion? Mind, 9, I88-205.

James, W. ( 1890$)$. Principles of psychology (2 Vols.). New York: Holt.

Lakoff, G. \& Kovecses, Z. (1983). The cognitive model of anger inherent in American English (Report n I0). Berkeley: Berkeley cognitive science program.

Lang, P..., Bradley, M.M., \& Cuthbert, B.N. (1990). Emotion, attention, and the startle reflex. Psychological Review, 97, 377-395.

Lange, C. (1885). Om sindsbevaegelser. Copenhagen: Kronar.

Leventhal, H. \& Scherer, K. (1987). The relationship of emotion to cognition: A functional approach to a semantic controversy. Cognition and Emotion, 1, 3-28.

Mandler, G. (1984). Mind and Body. New York: Norton.

Matsumoto, D. (1987). The role of facial responses in the experience of emotion: More methodological problems and a meta-analysis. Journal of Personality and Social Psychology, 52, 769-774.

Pennebaker, J.W. (1982). The psychology of physical symptoms. New York: Springer-Verlag.

Personality and Social Psychology Bulletin (1990). Special issue: Centennial celebration of the Principles of Psychology of William James. Personality and Social Psychology Bulletin, 16 597-734.

Philippot, P. \& Rimé, B. (1997). The perception of bodily sensation during emotion: A cross-cultural perspective. Polish Bulletin of Psychology, 28, 175- | 88. Reisenzein, R. (1983). The Schachter theory of emotion: Two decades later. Psychological Bulletin, 94, 239-264.

Philippot, P. \& Schaefer, A. (200 I). Emotion and memory. In T.J. Mayne and G.A. Bonano (Eds.), Emotion: current issues and future directions (pp. 82-122). New York: Guilford Press.

Philippot, P. (1992). Peripheral differentiation of emotion in bodily sensations, physiological changes, and social schemata. Unpublished doctoral dissertation. Department of Psychology University of Louvain, Belgium.

Philippot, P. (1993a). Inducing and assessing differentiated emotional feeling states in the laboratory. Cognition and Emotion, 7, 171-193.

Philippot, P. (1993b, September). Knowing what you feel: Biological and social determinants of bodily sensations during emotion. In F. Strack (Chair). Psychological foundations of 
phenomenological states. Symposium conducted during the General Meeting of the European Association of Experimental Social Psychology, Lisbon, Portugal.

Philippot, P., Chapelle, C. \& Blairy, S. (2002, in press). Respiratory feedback in the generation of emotion. Cognition \& Emotion.

Philippot, P., Rimé, B., Maric, M., Saldarelli, R. \& Feldman, R.S. (1990, June) Cultural and semantic determinants of the physiological changes reported in emotion. Paper presented at the general meeting of the European Association of Experimental Social Psychology, Budapest, Hungary.

Rimé, B. \& Giovannini D. (1986). The physiological patterning of emotional states. In K. R.Scherer, H. G. Wallbott \& A. B. Summerfield (Eds.), Experiencing emotion: A cross-cultural study (pp. 84-97). Cambridge: Cambridge University Press.

Rimé, B., Philippot, P., \& Cisamolo, D. (1990). Social schemata of peripheral changes in emotion. Journal of Personality and Social Psychology, 59, 38-49.

Ross, M. (1989). Relation of implicit theories to the construction of personal theories. Psychological Review, 96, 34I-357.

Schachter, S. (1964). The interaction of cognitive and physiological determinants of emotional state. In L. Berkowitz (Ed.), Advances in Experimental Social Psychology: Vol. I. (pp. 49-79). New York: Academic Press.

Scherer, K. R., Wallbott, H. G. \& Summerfield, A. B. (1986). Experiencing emotion: A cross-cultural study. Cambridge: Cambridge University Press.

Scherer, K.R. (1984a). On the nature and function of emotion: A component process approach. In K. Scherer and P. Ekman (Eds.), Approaches to emotion (pp. 293-317). Hillsdale, N.J:: Erlbaum.

Scherer, K.R. (199|). On social representation of emotional experience: Stereotypes, prototypes, or archetypes? In M. von Cranach, W. Doise \& G. Mugny, (Eds.), Social representations and the social bases of knowledge, Proceedings of the First Congress of the Swiss Society of Psychology. Bern: Huber.

Schwartz, G. E., Weinberger, D. A. \& Singer, J. A. (1981). Cardiovascular differenciation of happiness, sadness, anger and fear following imagery and exercice. Psychosomatic Medicine, 43, 343-364.

Shields, S.A. (1984). Reports of bodily change in anxiety, sadness and anger. Motivation and Emotion, 8, |-21.

Stemmler, G. \& Meinhardt, E. (1990). Personality, situation and physiological arousability. Personality and Individual Differences, II, 393-308.

Stemmler, G. (1992). The vagueness of specificity: Models of peripheral physiological emotion specificity in emotion theories and their experimental discriminability. Journal of Psychophysiology, 6, 17-28.

Stepper, S. \& Strack, F. (1993). Proprioceptive determinants of affective and nonaffective feelings. Journal of Personality and Social Psychology, 64, 21 I-220.

Wenger, M.A. (1950). Emotion as visceral action: An extension of Lange's theory. In M.L. Reymert (Ed.), Feelings and emotions. New York: McGraw-Hill. 


\section{Dario Galati}

Departement de Psychologie

Université de Turin

\section{LE LANGAGE DES ÉMOTIONS: LEUR DONNER UNE VOIX OU LES METTRE EN PAROLES?}

\section{Introduction}

Le but de ma communication est celui de présenter un compte-rendu des principaux thèmes qui émergent de la recherche sur le langage des émotions, orienté par quelques considérations concernant le rapport problématique existant entre le langage des émotions et l'expérience émotionnelle. Le premier point se référera à la distinction entre langage préverbal, c'est-à-dire les manifestations vocales qui expriment les émotions et le langage verbal, fait de paroles qui nomment, représentent et décrivent les émotions. Le second point concernera le thème aujourd'hui encore très débattu de la définition du terme émotion et du concept auquel il se réfère. Le troisième point se référera à des études sur le lexique émotionnel et illustrera les diverses propositions de classification qui en émergent. En conclusion, on présentera quelques considérations critiques concernantes la méthode de ces recherches ainsi que certaines hypothèses pour des nouvelles enquêtes dans ce domaine.

\section{Langage préverbal et verbal des émotions}

Le rapport entre langage et émotions va se poser de deux manières tout à fait différentes selon que l'on prend en considération les formes préverbales et prélinguistiques, c'est-à-dire les signaux vocaux qui accompagnent les émotions, ou le langage verbal, c'est-à-dire les paroles qui indiquent les émotions et qui sont organisées en différentes structures linguistiques. 
Dans le premier cas, le rapport est en quelque sorte direct, dans le sens qu'il se réalise sans médiation, étant donné que ce type de langage, notamment ses formes les plus simples et les plus immédiates, (Wundt, 1890, voir pour un approfondissement sur ce sujet Anolli \& Ciceri, 1992; Goffman, 1979; Poggi, 1981 et Scherer, 1993, 1994) font partie de l'expérience émotionnelle même. La médiation due à l'emploi des codes symboliques extérieurs à l'expérience émotionnelle commence à se manifester dans les formes symboliquement plus élaborées qui s'éloignent le plus de l'expérience directe puisqu'elles sont modelées par des codes linguistiques conventionnels et qu'elles sont utilisées comme «emblèmes vocaux» (Scherer, 1994). De toute façon, le lien de ces expressions vocales avec l'expérience n'est pas complètement coupé.

Dans le second cas, lorsqu'il s'agit du langage verbal et des paroles qui indiquent les émotions, le rapport entre langage et expérience se fait bien plus faible et plus problématique, car tout lien direct est perdu et par conséquent aussi, l'enracinement du langage dans l'expérience. En effet, les paroles qui indiquent les émotions n'ont rien à faire avec l'expérience émotionnelle puisqu'elles tirent leur signification d'un système linguistique qui assigne à chaque mot un domaine sémantique délimité et systématisé en forme conceptuelle.

Cette différence se fait encore plus évidente si l'on pense aux fonctions spécifiques des deux types de langage. Les fonctions du langage préverbal des émotions, c'est à dire des signaux vocaux des émotions, ont été schématisées et résumées récemment par Scherer (1992; 1993). Partant des modèles de Brunswik et de Bulher (cités par Scherer 1992) il distingue trois fonctions principales: la fonction expressive par laquelle ils manifestent l'expérience, celle symbolique, par laquelle ils se réfèrent à des situations ou à des objets et enfin la fonction d'appel par laquelle le sujet

58 communique avec les autres, leur signalant la situation ou leur demandant d'intervenir.

Bien différente est la fonction du langage verbal qui sert surtout à représenter l'expérience émotionnelle dans une forme systématique, analytique et logique. Ce type de langage a une fonction qui a moins à voir avec l'expérience des émotions et qui concerne surtout la représentation en forme logique de la connaissance des émotions mêmes. En ce sens le langage verbal est quelque chose d'autre par rapport à l'expérience qu'il veut représenter. 
Parler du langage verbale, c'est à dire des paroles que nous employons pour indiquer les émotions, c'est donc poser un problème qui se réfère aux moyens symboliques dont nous disposons pour les représenter et à la fin se référer à la capacité de le faire dans une forme réaliste, si ce n'est objective.

\section{Le mot et le concept d'émotion}

Le problème du rapport entre langage et expérience émotionnelle se pose à partir du terme même d'émotion et de sa définition. Dès les débuts de la psychologie scientifique, les chercheurs qui se sont occupés d'émotions, se sont posé le problème d'éclairer la signification de ce terme, en spécifiant la structure et le contenu du concept auquel il se réfère. L'exigence d'un éclaircissement linguistique et conceptuel servait et sert à identifier un ensemble de phénomènes qui constituent, d'un côté le référent objectif de ce terme, de l'autre le champ des objets empiriques de la recherche de la psychologie des émotions.

A la différence d'autres termes de la psychologie tels que mémoire, pensée, raisonnement, le terme émotion a toujours été particulièrement difficile à définir, probablement parce que la structure du langage verbal ressemble beaucoup plus à celle de la pensée logique, en particulier de celle propositionnelle qu'à celle de l'émotion. Cette difficulté a déjà été mise en évidence par James (1890) qui soutenait que la multiplicité et la variété infinie des expériences émotionnelles ne peut se réduire aux distinctions prévues par le langage. Des peuples différents ont introduit des mots divers pour exprimer différents aspects des émotions, sans jamais réussir à donner une représentation exhaustive du phénomène.

C'est pour cela peut-être qu'il est difficile d'arriver à une définition univoque du terme, comme le démontrent les définitions nombreuses et diverses que plusieurs auteurs et plusieurs courants psychologiques ont données de ce terme. Ces tentatives ont joué un rôle de multiplicateur des significations du mot émotion tellement évident que Kleinginna et Kleinginna (Kleinginna \& Kleinginna, 198I) ont pu identifier une centaine de définitions possibles et différentes du terme et du concept émotion.

On a proposé différents types de solutions pour sortir de la confusion. Une solution extrême est celle introduite par Duffy (194I) qui proposa de 
faire disparaître de la scène de la psychologie scientifique le terme et le concept «émotion» en le confinant dans le cadre de la naïve psychologie populaire et en proposant l'interprétation des phénomènes émotionnels uniquement en termes d'activation physiologique.

Tous les psychologues contemporaines des émotions généralement refusent cette solutions en proposant, à partir de Lazarus (1980), de considérer le concept d'émotion comme instrument utile à identifier un ensemble d'objets empiriques qui peuvent être soumis à l'enquête psychologique et qui ne se réduisent pas seulement à la physiologie ou au comportement. Mais au delà de cet accord, les chercheurs n'ont pas réussi à sortir de la Babel des définitions.

Une solution récente du problème permet de considérer le mot et le concept d'émotion comme légitimes et utilisables bien que leur définition ne puisse être claire et évidente comme celles des concepts classiques. C'est la solution que proposent ceux qui soutiennent que le concept d'émotion a une structure prototypique. On sait que ces auteurs (Fehr \& Russell, 1984; Shaver, Swartz, Kirson \& O'Connor, 1987), se référant au modèle de Rosch (1978), croient que les difficultés que l'on rencontre lorsqu'on essaie de définir l'émotion ne dérivent pas du manque d'un champ sémantique spécifique qui peut se rapporter à ce mot, mais du fait que ce champ a des confins plutôt flous, ce qui ne permet pas d'identifier la signification du mot émotion ayant une forme précise et non équivoque, liée à la présence ou à l'absence d'une quantité nombrable d'attributs nécessaires et suffisants. On peut toutefois définir le concept d'émotion comme prototype, c'est-à-dire en termes de bons ou mauvais exemples partageant une quantité variable d'attributs communs à la catégorie même, et ayant entre eux un rapport hiérarchique. Au sommet de la hiérarchie se trouve le mot émotion surordonné à tous ses exemples, au niveau intermédiaire les exemples plus typiques, qui sont quantitativement limités, et au niveau inférieur, les exemples moins typiques, bien plus nombreux et groupés en divers ensembles subordonnés aux différents exemples typiques et dont les termes sont liés entre eux par une «ressemblance de famille» (Fehr \& Russell, 1984). On a souvent associé le modèle, prototypique à celui de script, présenté comme l'instrument conceptuel utilisé pour mieux représenter les aspects dynamiques de l'expérience émotionnelle (Fischer, 1991, Fischer \& Frijda, 199|). On sait que le script (Schank \& Abelson, 1977) a été présenté comme une stratégie de la mémoire utilisée pour mémoriser des événements et 
des actions qui se déroulent selon une séquence de phases plus ou moins typique et constante. Comme le prototype, le script également est un schéma de représentation qui n'est pas rigide, étant constitué d'éléments qui ne sont pas nécessaires et suffisants pour le définir, mais qui se réduisent seulement à une séquence d'actions possibles plus ou moins typiques et, dans une certaine mesure, susceptibles de varier.

Un autre modèle qui présente l'émotion comme la représentation d'un schéma d'action est celui de prototype introduit par Lang (1984). Bien que le terme prototype semble se rapprocher plus de la définition structurale de l'émotion de Fehr et Russell, il est en réalité beaucoup plus proche des modèles qui décrivent l'émotion en termes de script. En effet, il définit l'émotion comme une tendance à l'action liée à l'activation d'un enchaînement structuré d'informations emmagasinées dans la mémoire.

Les modèles de prototype et de script ont obtenu un grand succès auprès des psychologues des émotions, donnant lieu à de nombreuses recherches conduites en différents contextes culturels (pour une panoramique récente des études voir Russell, Fernández-Dols, Manstead \& Wellenkamp, 1994), la définition d'émotion qui en est dérivée n'a toutefois pas réussi à conquérir l'unanimité. Certains auteurs (Armstrong, Gleitman \& Gleitman, 1983; Barsalou, 1987; Ortony, Clore \& Foss, 1987) ont en effet avancé des critiques plus ou moins sévères et radicales aux définitions prototypiques au point de nier qu'elles soient en mesure d'exprimer la signification du concept d'émotion tel qu'il apparait d'après l'analyse de l'expérience émotionnelle (Stein \& Trabasso, 1922). Compte tenu des argumentations alléguées par les différents auteurs, les principales critiques faites à cette théorie sont les suivantes:

I) L'observation selon laquelle les philosophes et les psychologues n'ont jamais pu trouver un accord pour définir le concept d'émotion n'a aucune valeur probatoire. Cet accord manqué ne fait que témoigner d'une difficulté, mais non d'une impossibilité à arriver à une définition classique (Ortony, et al., 1987).

2) II y a un manque de méthodologie dans les recherches sur les prototypes: les consignes données aux sujets dans les différents types d'expérience empêchent de vérifier s'ils possèdent un concept classique d'émotion. En effet, on ne leur demande jamais d'essayer de définir l'émotion en termes généraux et non équivoques, mais simplement d'énumérer des exemples de ce concept, prenant les premiers de la liste comme les meilleurs 
exemples, les derniers comme les pires d'une structure d'organisation que l'on suppose hiérarchique. Une consigne de ce type permet de vérifier seulement si ce qu'affirme l'hypothèse (la théorie prototypique) est vrai, mais elle n'exclue pas que d'autres affirmations différentes et absentes de l'énoncé de l'hypothèse (la définition classique) soient également vraies.

Armstrong, Gleitman et Gleitman ont démontré que certains concepts qu'il est possible de définir de manière classique (numéro un, numéro impair, figure géométrique plane) peuvent être également représentés par une structure prototypique. Barsalou (1987) estime que cette dernière est une caractéristique de tous les concepts. Selon Armstrong et ses collaborateurs, il faut faire une distinction entre le sens ou signification d'un concept, qui pour les concepts que l'on peut définir de manière classique doit être identifié dans un ensemble précis d'attributs nécessaires et suffisants, et la structure prototypique. Cette dernière peut n'avoir rien à voir avec la définition logique du concept, mais elle peut avoir seulement une fonction auxiliaire, liée à la tâche pragmatique de reconnaître dans la réalité des exemples concrets de ce concept (Armstrong et al., 1983).

La conclusion de toutes ces argumentations porte à considérer la définition d'émotion en termes classiques comme possible et nécessaire, assignant cette tâche aux futures recherches sur ce sujet (Ortony, et al., 1987).

Un modèle qui découvre des critères précis pour définir l'émotion et en déduit une théorie des émotions primaires est celui de Johnson Laird et Oatley (Oatley \& Johnson-Laird, 1987; Johnson-Laird \& Oatley, 1989). Selon cette «théorie communicative des émotions», toute l'expérience émotionnelle humaine est formée de myriades de variations que l'on peut ramener à 5 modalités fondamentales ou émotions primaires: joie, tristesse, colère, peur, dégoût (happiness, sadness, anger, fear, disgust). Chaque émotion primaire est fondée sur un signal non propositionnel provoqué par l'évaluation cognitive d'une situation, qui s'adresse à l'intérieur et à l'extérieur de l'organisme. Ce signal non propositionnel est cet état particulier de la sensation qui accompagne chaque émotion et en est la particularité, et que nous indiquons avec des mots du type colère, peur, joie, tristesse, dégoût. Le caractère non propositionnel de ce signal permet une transmission très rapide de l'information et par conséquent une réponse rapide et adéquate en termes d'adaptation et qui en grande partie est programmée et automatique. 
Ce qui caractérise une émotion primaire et lui attribue sa qualité distinctive, c'est la présence d'un de ces signaux prépropositionnels, entendus comme qualia non décomposables, primitifs et autosignifiants, enfin impossibles à définir en termes propositionnels.

Selon ce modèle on ne peut définir ni décrire les émotions primaires en termes de catégories prototypiques. Ce que l'on peut représenter à travers un prototype, c'est l'ensemble de causes et d'événements concomitants d'une émotion de base, mais non l'émotion même. La définition implicite d'émotion qui en résulte a donc certains aspects en commun avec la définition prototypique, mais elle est peut-être plus proche de celle classique du moment qu'elle estime qu'on peut différencier les émotions de manière absolument certaine à partir de la présence d'un des cinq signaux prépositionnels.

\section{Une définition ouverte d'émotion}

C'est une position différente par rapport aux précédentes que prend Paul Ekman (1984, 1992 a, b): il propose une série définie de critères empiriques et pas seulement logico-formels pour distinguer ce qui est émotion de ce qui ne l'est pas, déplaçant la discussion du niveau des concepts à celui des données empiriques. Les critères de détermination qu'il propose sont en étroite relation avec sa théorie des émotions primaires puisqu'ils ne sont rien d'autre que les principales caractéristiques de ces dernières. On reconnaît comme primaires les émotions qui ont un clair enracinement biologique et phylogénétique révélé par la présence de caractéristiques, au nombre tantôt de 10 (Ekman, 1984), tantôt de 9 (Ekman, 1992a), que l'on peut relever empiriquement et qui sont en dernière analyse celles qui définissent le domaine même des phénomènes émotionnels. Nous citons de suite la liste la plus récente de ces caractéristiques:

I. Présence de signaux non verbaux distincts

2. Présence chez d'autres primats

3. Réactions physiologiques distinctes

4. Présence d'événements antécédents distincts et universels

5. Cohérence entre les réponses émotionnelles

6. Apparition rapide 
7. Brève durée

8. Évaluation cognitive automatique

9. Occurrence spontanée

Ce que Ekman souligne souvent et affirme avec force, c'est que l'émotion, avant d'être un concept, est un fait d'expérience; aussi retient-il que tous les problèmes qui lui sont reliés peuvent se résoudre beaucoup plus facilement au niveau empirique qu'au niveau du débat exclusivement théorique (Ekman, 1992 b).

Par cohérence avec cette perspective «réaliste», les 9 caractéristiques ne sont pas présentées, en termes logiques, comme des conditions nécessaires et suffisantes et donc exhaustives de la définition des émotions, mais comme des critères empiriques indispensables pour identifier et délimiter un champ de recherche. Comme telles, elles ne prétendent pas être exhaustives, aussi leur nombre pourra augmenter lorsque seront suffisamment évidentes d'autres caractéristiques partagées par les émotions de base.

\section{Dimensions et catégories du lexique émotionnel}

Les recherches sur le concept d'émotion focalisent leur attention sur le problème des confins et des caractéristiques qui le définissent; les nombreuses recherches qui ont étudié les structures du lexique émotionnel dans différentes langues, au contraire, ont focalisé leur attention sur l'analyse des rapports qui lient entre eux les différents termes au delà de leurs relations hiérarchiques.

Les travaux de ce type furent sans doute stimulés par les recherches conduites par Osgood et ses collaborateurs (Osgood, Suci, \& Tannenbaum, 1957) sur les dimensions de significations des langues naturelles utilisant le différentiel sémantique. Les recherches initiales de Osgood firent émerger trois dimensions principales de signification des langages naturels: évaluation, activité et puissance qui furent interprétées comme des dimensions de caractère affectif (Osgood, 1969). L'évaluation se réfère à la dimension plaisir-déplaisir, l'activité à l'activation de l'organisme, la puissance à la capacité de la part du sujet de maitriser la situation où il se trouve.

Plusieurs auteurs appliquèrent la technique du différentiel sémantique au lexique des émotions, pour en étudier les dimensions spécifiques. C'est ainsi que Averill (1975) étudia les corrélations entre les scores obtenus par des 
termes émotionnels sur différentes échelles du différentiel sémantique, faisant émerger l'existence de quatre dimensions. Les premières et les plus importantes étaient celles du ton hédoniste et de l'activation. Russell et Merhabian (1977), utilisant le même instrument, confirmèrent l'existence des trois dimensions que Osgood avait déjà identifiées.

A travers une recherche complexe qui partait de l'exigence de mettre de l'ordre dans le langage des émotions, à travers la construction d'un dictionaire des termes émotionnels, Davitz (1969) arriva à mettre en évidence quatre dimensions (activation, relation, ton hédoniste et compétence), en soumettant à une analyse factorielle les corrélations entre les scores de ressemblance entre les termes de son dictionaire.

Beaucoup des recherches faites au cours des années suivantes utilisèrent comme instrument d'analyse des rapports de ressemblance entre les termes, la technique du scaling multidimensionnel à partir du travail de Bush (1973) qui identifia trois dimensions: ton hédoniste, activation, niveau d'activation dont seulement les deux premières expliquaient plus de $80 \%$ de la variance totale du phénomène. L'existence de ces deux dimensions principales a généralement été confirmée par de nombreuses autres recherches qui ont utilisé le scaling multidimensionnel (Neufeld, 1957, 1975; Russell, 1978). Plus récemment Watson et Tellegen (1985) ont confirmé l'hypothèse bifactorielle, en analysant les résultats différents de certaines recherches où émergent de nombreux et différents facteurs organisateurs du lexique émotionnel. De nombreuses recherches ont mis aussi en évidence le caractère bipolaire de ces dimensions (Bush, 1973; Lunberg \& Devine, 1975; Neufeld, 1975, 1976; Stone \& Coles, 1970).

Même si les recherches faites avec le scaling multidimensionnel ou avec l'analyse factorielle obtiennent des résultats similaires sur le thème de la bidimensionalité ou de la bipolarité des structures des lexiques émotionnels, elles ont été souvent menées de manière a-théorique, c'est-à-dire sans se référer explicitement à un modèle de représentation de la connaissance des émotions. II ne manque pourtant pas de recherches plus articulées et plus complexes qui ne se limitent pas à étudier les dimensions du lexique, mais encadrent ce thème dans le plus vaste problème des structures générales du langage des émotions et de la signification que chaque terme y acquiert.

Je me limiterai à rappeler ici quelques-unes de ces recherches particulièrement importantes pour leur richesse théorique et pour l'influence qu'elles ont exercée dans le cadre de la psychologie des émotions. 
Une d'entre elles est sans doute le travail de pionnier de Davitz (1969) qui, comme nous l'avons déjà signalé, constitue une première tentative de construire un dictionnaire de termes émotionnels de la langue anglaise, contenant des définitions claires et précises de chaque mot, fondées sur l'analyse de définitions obtenues à partir d'un échantillon de personnes parlant cette langue. Cette tentative est une sorte de défi contre les croyances pessimistes des béhavioristes sur la possibilité d'exprimer l'expérience émotionnelle en termes linguistiques. Davitz ne partage pas cette opinion puisqu'à son avis une langue comme l'anglais peut être en mesure d'exprimer l'expérience émotionnelle avec une approximation acceptable, à condition que l'on mette de l'ordre dans le langage des émotions; et cela en construisant un dictionnaire composé d'un certain nombre de termes qui puissent être définis avec précision par un ensemble nombrable d'attributs ou d'éléments signifiants discrets, typique et spécifique pour chaque parole. Le modèle théorique qui le guide est celui qu'il appelle «la métaphore atomistique», une conception du lexique émotionnel qui lui a été suggérée par Mowrer. Selon cette conception, la définition de chaque terme émotionnel doit être considérée et construite comme un composé défini de nombreux éléments d'information. Chacun des éléments peut être présent dans la définition de plusieurs émotions, mais comme faisant partie de configurations toujours différentes entre elles.

Un autre exemple d'un approche plus théorique à l'étude du lexique émotionnel est représenté par les recherches de Russell et de ses collaborateurs (Russell, 1978, 1979, 1980, 1983, 1991; Russell, Lewicka \& Niit 1989; Russell \& Mehrabian, 1977) et de Plutchik (Plutchik, 1980 a, b; 1991; Conte \& Plutchik, 1981).

Partant d'un intérêt initial pour les dimensions du langage des émotions que, d'après lui, on peut ramener à deux axes polaires principaux (celui de

66 I'activation et du plaisir/déplaisir), Russell en vient à esquisser un modèle plus complexe où le langage des émotions est ramené aux structures de la connaissance émotionnelle. Les résultats de ses recherches, conduites selon la méthode du scaling multidimensionnel, mettent en évidence, à son avis, que les dimensions du lexique affectif sont reliées de manière très complexe. Ces relations peuvent être représentées à travers un modèle circulaire où les concepts des émotions se disposent le long du périmètre d'un cercle selon un ordre bien défini qui reste constant au-delà des différences subjectives. II s'agit du modèle circomplex modifié par les travaux de 
Schlosberg $(1952,1954)$ et que Russell propose comme en mesure d'exprimer la structure du lexique émotionnel, celle de l'expérience affective telle qu'elle émerge de l'analyse des comptes-rendus verbaux, et plus en général, de la structure de la connaissance que l'homme commun a des émotions. Selon Russell (1980), ce modèle est en mesure d'identifier trois propriétés fondamentales du langage et plus en général de la représentation cognitive de l'émotion: I) la première se réfère au fait que l'axe d'agréabledésagréable et celui de l'activation sont les organisateurs principaux de ce domaine; 2) la seconde au fait que ces axes sont bipolaires, raison pour laquelle tous les termes et tous les concepts émotionnels peuvent être représentés comme des degrés de variation d'intensité compris entre leurs extrêmes; 3) la troisième propriété dérive de l'ordonnance circulaire et réside dans le fait que chaque terme et chaque concept émotionnel, occupant une certaine position dans l'espace par rapport aux deux axes, peut être défini comme une espèce de combinaison de plaisir et d'activation et peut donc être identifié avec précision au point d'intersection de deux coordonnées. La conclusion a des aspects semblables et d'autres différents de celle de Davitz. Pour ce dernier, en effet, la signification d'un terme est un agrégat défini d'éléments divers; pour Russell, elle dérive de sa position par rapport aux organisateurs de la signification des émotions. Ce que les deux conceptions ont en commun, c'est de toute manière le fait qu'elles conçoivent un terme (et sa signification) comme une entité discrète et que l'on peut précisément définir de manière atomistique ou spatiale.

Plutchik a également utilisé le modèle circomplex comme instrument utile autour duquel il organise une théorie des émotions articulée et complexe, applicable aussi bien au lexique qu'aux structures émotionnelles de la personnalité et de ses développement pathologiques possibles. Dans ses recherches sur le langage des émotions, il paraît plus intéressé, à la différence de Russell, à en faire émerger une forme d'organisation catégorielle plus que dimensionnelle. Le modèle circomplex permet de mettre en évidence, selon Plutchik, non seulement les rapports de continuité entre les termes, mais aussi leur discontinuité. Les termes en effet créent des regroupements que l'on peut bien identifier avec les huit émotions que l'auteur indique comme primaires (fear, anger, joy, sadness, acceptance, disgust, anticipation, surprise). Ainsi une forme d'organisation linguistique et conceptuelle est proposée pour soutenir l'existence de structures analogues que l'on suppose exister dans le cadre de l'expérience réelle de l'émotion. 
Un exemple ultérieur de recherche sur le lexique fondée théoriquement, mais différente des travaux illustrés jusqu'ici est celui de Johnson-Laird et Oatley (1989) qui est étroitement relié à leur «théorie communicative des émotions». Cette théorie implique que la signification de chaque terme émotionnel puisse être ramenée à une des ces cinq modalités d'émotions de base. Cette prédiction théorique est démontrée empiriquement à travers une analyse sémantique de 590 termes émotionnels anglais, faite par les auteurs mêmes, qui démontrent que tous les termes examinés peuvent être ramenés aux cinq émotions de base, à travers une série de «questions diagnostiques» concernant la signification de chaque terme. L'approche méthodologique de cette recherche est sans aucun doute originale et innovatrice puisqu'elle rompt la tradition des travaux fondés sur l'analyse extrêmement variée des rapports de ressemblance entre les termes, faisant référence à un modèle théorique explicite qu'elle veut démontrer. Actuellement, toutefois, cette méthode semble trop liée au jugement subjectif, comme l'admettent en partie les auteurs mêmes lorsqu'ils reconnaissent que les conclusions auxquelles elle aboutit devraient être vérifiées par le jugement de personnes anglophones. Jusque-là, il est légitime de soupçonner que les résultats soient, sinon déduits de la théorie, du moins fortement influencés par elle.

Toutes les recherches illustrées jusque ici se réfèrent à la langue anglaise et prétendre les généraliser impliquerait un choix non seulement logocentrique, mais aussi anglocentrique. Pour éviter ces attitudes il faut réaliser, de manière systématique, des recherches transculturelles utilisant des méthodes rigoureuses et comparables. Certaines d'entre elles ont déjà été réalisées par Russell lui-même (Russell, 1983; Russell, Levicka \& Niit, 1989) qui a traduit les termes utilisés dans ses analyses précédentes du lexique anglais en japonais, en chinois, en croate, et dans la langue des Gujarites demandant à des sujets parlant ces langues de regrouper ces termes sur la base de leur ressemblance. Le scaling multidimensionnel a mis en évidence une disposition circulaire des termes qui se maintient constante dans toutes les langues analysées et qui est analogue à celle du lexique anglais. La limite de cette recherche réside toutefois dans le fait qu'elle a employé des traductions des termes, sans être suffisamment garantie que la traduction corresponde, n'ayant pas, par exemple, contrôlé la réelle équivalence des termes par rapport à leur fréquence d'emploi. A propos de la difficulté de traduire d'une langue à l'autre des mots qui indiquent l'émotion, Van Goozen 
et Frijda (Van Goozen \& Frijda, 1993) ont fait récemment une recherche sur l'équivalence des mots les plus communs indiquant les émotions dans différentes langues occidentales (anglais, français, italien, hollandais). Cette recherche a démontré qu'il n'y a pas beaucoup de mots qui aient la caractéristique de posséder la même signification en même temps que d'être utilisés avec la même fréquence dans les différentes langues. Et plus précisément elle a mis en lumière comment les problèmes d'équivalence et de possibilité de traduction semblent s'appliquer dans une mesure plus marquée au lexique anglais et dans une mesure inférieure aux autres langues étudiées. Cette donnée est sûrement importante et les auteurs la proposent comme un avertissement pour la recherche transculturelle vu que l'on utilise l'anglais comme la base plus commune pour les taxonomies des émotions.

Une certaine similarité structurale entre le lexique émotionnel anglais et celui du philippin a été mise en évidence dans une récente recherche qui n'a pas utilisé des termes traduits de l'anglais, mais ce résultats n'ont pas été confirmés par ceux d'autre et plus nombreux travaux sur des langues différentes de l'anglais (Church, Katigbak, Reyes, \& Jensen, 1998). Une étude de Gehm et Scherer (1988) sur le lexique émotionnel allemand n'a pas confirmée la disposition circulaire des termes prévue par le modèle circomplex; en outre elle a mis en lumière des différences encore majeures par rapport à ce modèle bidimensionnel: les regroupements de mots qui émergent de l'analyse des termes allemands ne semblent pas se référer aux émotions de base, mais à d'autres matrices de signification que les auteurs interprètent à la lumière de la théorie des contrôles d'évaluation du stimulus énoncée par Scherer (1984).

En analysant de manière comparative des échantillons plutôt étendus de mots tirés des lexiques émotionnels des langues néolatines (italien, français, espagnol, catalan, roumain), Galati et ses collaborateurs (Galati, 1986; Galati, Sini, 1998a, 1998b; Galati, Sini, Estaun Ferrer, Soler Vilageliu, Mateos, 1998, Galati Tinti, Belghiru, Sini, in presse) ont démontré, utilisant des processus d'analyse semblables à ceux utilisés par Plutchik et par Russell, comment existent certaines particularités dans les rapports entre les termes communs aux langues néo-latines et que ne relèvent pas entièrement les résultats des analyses de Russell ou de Plutchik citées plus haut. On confirme le développement circulaire de la distribution, mais non la quantité, le contenu et le rapport réciproque entre les regroupements que les résultats des recherches qui se réfèrent à l'anglais ont mis en évidence. Même la structure 
bidimensionelle n'est pas entièrement vérifiée puisqu'émergent clairement dans les deux langues l'axe de la valence hédoniste, mais moins clairement celui de l'activation qui est remplacé généralement par un axe que l'on peut référer au coping

\section{Conclusions}

Après avoir terminé le compte rendu des études sur le langage des émotions, on peut faire quelques considérations critiques à propos du traitement imposé par le langage à l'expérience émotionnelle. La première concerne le problème de la signification du terme et du concept d'émotion et peut être exprimée ainsi: «est-il raisonnable de supposer que le terme et le concept d'émotion identifient, de manière claire et sans équivoque, un cadre de phénomènes d'expérience qui ont vraiment les caractéristiques que leur attribuent les formes symboliques utilisées pour les représenter?». La réponse à cette question reste problématique. II n'y a pas encore d'accord sur une définition univoque d'émotion et sur le cadre de phénomènes à l'intérieur desquels la recherche devrait être circonscrite. Deux positions principales (la conception prototypique et celle classique) se font face, délimitant et décrivant de manière différente le champ sémantique du terme.

II est sûrement raisonnable de penser, comme le suggère Ekman (I992 b), que, pour résoudre ce problème, il faut déplacer l'attention des chercheurs du contexte purement conceptuel où restent souvent confinés leurs travaux sur la question, à celui de l'expérience concrète des émotions, observées et étudiées avec des méthodes empiriques et expérimentales. II est toutefois également vrai que toute la recherche empirique sur les

70 émotions (les recherches sur les expressions, sur la physiologie, sur les antécédents, ou sur les systèmes d'évaluation cognitive des émotions) utilisent les catégories du lexique émotionnel pour identifier et spécifier les objets de leur analyse, souvent sans se demander de manière explicite si les distinctions linguistiques correspondent aux différentes expériences. II est donc évident que les recherches empiriques ont besoin d'éclaircir préalablement le champ de phénomènes qu'elles doivent considérer et que les études sur ce que l'on entend lorsque l'on parle d'émotion sont sûrement utiles pour réaliser cet éclaircissement. 
Au-delà des différences entre les diverses significations attribuées au terme émotion, on peut cependant faire remarquer une ressemblance de fond entre les différentes positions: elles reconnaissent toutes que lorsqu'on parle d'émotion, on se réfère à un champ de phénomènes réellement existant et unitaire, que ce seul terme peut suffire à représenter de manière adéquate.

Mais, à ce propos, nous sommes portés à réfléchir par les données de certaines recherches ethnographiques dont Russell (1991) nous offre une revue que nous allons synthétiser et qui semblent mettre en doute cette croyance générale. II résulte de ces recherches que le mot émotion n'a pas en effet un équivalent précis dans toutes les langues connues. Certaines données tirées des recherches ethnographiques citées (Howell, 1981) soulignent en outre comment dans certaines cultures l'émotion ne se distingue pas en tant que catégorie autonome, mais est plutôt assimilée à d'autres formes d'expérience mentale. Comme l'observe Russell, la possibilité que l'émotion ne se réfère pas à un domaine spécifique de phénomènes reconnu dans toutes les cultures, est peut-être le résultat le plus important obtenu des comptes-rendus ethnographiques à propos des émotions. En effet l'absence, dans certaines langues, du terme émotion porte à penser que le vaste domaine des phénomènes que nous représentons de manière unitaire et distincte pourrait «peut-être être» décrit de manière non unitaire et avec des articulations et des rapports différents par rapports aux autres phénomènes psychiques.

On peut étendre en grande partie les considérations critiques faites à propos du terme et du concept d'émotion aux recherches sur le lexique des émotions. Dans ce cas également, en effet, on est en droit de se demander dans quelle mesure les distinctions lexicales sont universelles et dans quelle mesure elles se réfèrent à de réelles distinctions dans le cadre de l'expérience. Fehr et Russell (1984) et Russell (1987) soulignent eux aussi comment il est nécessaire de ne pas confondre les caractéristiques de l'ordre linguistique conceptuel avec celles qui appartiennent à l'expérience. D'après Frijda et Zammuner, «étiqueter les émotions est le résultat d'un processus constructif de réflexion qui transforme l'expérience perceptive en expérience intérieure, apportant ses propres modifications» (Frijda \& Zammuner, 1992, p. 389). Ne pas tenir compte de l'écart entre langage et expérience porte à une position logocentrique qui, donnant un crédit excessif au langage, accepte, de manière plutôt a-critique, ses distinctions et ses articulations 
comme des indicateurs utiles et dignes de foi de différenciations analogues dans le cadre de l'expérience. Quant aux résultats des recherches transculturelles sur les lexiques émotionnels, il est évident qu'ils ne sont pas entièrement d'accord entre eux en ce qui concerne la quantité de dimensions ou de catégories du langage des émotions et tout cela rend très improbable l'hypothèse d'une classification linguistique universelle des émotions.

Au delà du problème du relativisme culturel des classifications linguistiques des émotions, il en existe d'autres qui peuvent constituer une sérieuse limite des recherches menées sur le lexique. Ces problèmes regardent I) le choix des mots utilisés dans les diverses enquêtes et 2) le crédit peut-être excessif prêté aux techniques d'analyse fondées sur le scaling multidimensionnel. Souvent les mots soumis à la procédure de scaling ne sont pas très nombreux ou ne représentent pas fidèlement tout le lexique dont ils sont tirés. D'autres fois le choix peut être, de manière aprioriste, fonctionnel pour mettre en évidence certaines dimensions comme celles classiques de l'activation ou de l'agréable/désagréable. Ces limites caractérisent, entre autres, les recherches de Russell et collaborateurs qui n'ont porté que sur 28 mots du lexique anglais.

En ce qui concerne la validité du scaling multidimensionnel et de procédures analogues plus variées, il est utile de rappeler qu'elles sont employées correctement si on les utilise dans une phase exploratrice de la recherche puisqu'elles ne donnent pas lieu à des résultats conclusifs, mais qui indiquent le mouvement général d'un phénomène et que l'on doit les interpréter à la lumière d'un modèle théorique. C'est pour cela que l'on peut difficilement les prendre comme fondement sur lequel construire un modèle (Trentin, 1988). Donner un nom aux dimensions qui émergent d'une analyse multivariée est une question de goûts où les convictions théoriques

72 interviennent peut-être plus que l'évidence empirique (Gehm \& Scherer, 1988).

Les résultats contrastants des nombreuses recherches sur la signification du mot émotion de même que sur la signification et les dimensions structurales des termes qui constituent les lexiques émotionnels des langues jusqu'ici examinées, portent à croire que l'on ne peut pas, pour le moment, identifier une certaine définition d'émotion ou une certaine classification du lexique émotionnel d'une certaine langue sur laquelle il y ait l'accord unanime des chercheurs. A mon avis cela ne doit pas décourager, mais au contraire inciter à de nouvelles recherches menées de manière plus organisée, 
homologuant le plus possible instruments et méthodes pour rendre possible la comparaison des résultats.

Une proposition intéressante pour sortir du relativisme et des malentendus linguistiques a été formulée par Wierzbicka (1992) qui propose, pour exprimer les émotions d'une manière qui soit universellement compréhensible et traduisible, la construction d'un langage extrêmement simplifié qui utilise des semantic primitives, c'est-à-dire des mots qui se réfèrent à des objets, des actions, des pensées très communs et qui ont un équivalent dans les contextes linguistiques et culturels les plus différents. On exprimerait avec ces mots les composantes plus simples de la signification d'une émotion qui pourrait ensuite être expliquée par une syntaxe élémentaire et une structure expositive de type narratif expliquant les circonstances qui donnent lieu à l'émotion et les réactions de l'individu. La proposition est certainement appréciable, mais les critiques ne manquent pas, comme celle avancée par Oatley et Johnson-Laird (1989), qui soutiennent que la représentation de l'émotion proposée par l'auteur comprend seulement l'analyse des conditions dans lesquelles l'émotion même peut être exprimée, comme Frijda (1986) l'avait déjà mis en évidence. Toutefois elle ne comprend aucun élément référé à l'expérience subjective qui est un aspect constitutif et fondamental de l'expérience émotionnelle.

L'approfondissement de la recherche sur le langage des émotions, malgré les difficultés qu'elle présente, est sûrement nécessaire puisqu'il n'y a pas, comme nous le disions plus haut, d'enquête empirique qui ne l'utilise pour identifier ses données, les analyser et en communiquer les résultats.

S'il n'est pas possible de sortir de ces limites, liées à la fonction représentative et symbolique du langage, qui en partie modèlent et transforment l'expérience émotionnelle, il est cependant possible d'en prendre conscience pour éviter de s'empêtrer dans les formes de représentation plus rigides, liées davantage à des croyances et des attitudes culturelles largement partagées et qui trouvent leur expression plus dans les formes du langage que dans des aspects réels de l'expérience. Pour atteindre cet objectif, l'analyse détaillée des éléments de signification des termes émotionnels élaborée par Davitz et, de manière différente également par Wierzbicka, ne suffit pas.

Pour mieux comprendre la signification du langage des émotions utilisé dans un certain contexte culturel il faut faire émerger ses prémisses idéologiques implicites qui participent à la construction du sens des mots qui 
le constituent. Par exemple, si l'on veut savoir ce qu'est la joie, il ne suffit probablement pas de savoir que dans une certaine langue le mot joie est caractérisé par n éléments de signification acceptés de manière consensuelle, mais il faut comprendre pourquoi on a été d'accord pour choisir ce nombre d'éléments pour construire la signification du concept de joie.

C'est précisément cet exemple qui me suggère une réflexion sur une donnée plutôt évidente et intéressante, mais peu commentée, et qui émerge fréquemment des recherches sur le lexique des émotions dans les langues occidentales: il s'agit de l'évidente disproportion numérique entre les termes qui indiquent des émotions positives et ceux qui indiquent des émotions négatives, disproportion qui en général est nettement au profit des émotions négatives (Galati 1986; Gius, Cozzi \& Spagnoletto, 1992). À ce sujet, on peut se demander la raison d'une telle disproportion et on peut imaginer deux solutions possibles. Dans le premier cas, les possibilités majeures de représentation que de nombreuses langues offrent aux émotions négatives ont un fondement réel dans l'expérience et reflètent leur plus grande «utilité» pour la survie de l'individu. Dans les seconds cas, cette disproportion serait plutôt liée à la possibilité d'une attitude «de pénitence» de la culture occidentale qui regarde avec méfiance les modalités d'expérience liées au plaisir et à la joie et leur réserve donc peu de place dans ses formes de représentation linguistique de l'expérience.

Affronter des problèmes de ce type demande que l'on adopte une attitude spéculative et de conscience majeure des implications théoriques et idéologiques des modèles que l'on utilise et des résultats que l'on obtient. II s'agit d'une tâche ardue, mais aussi certainement utile pour le progrès de la recherche dans le cadre de la psychologie des émotions.

\section{RÉFÉRENCES}

Anolli, L. \& Ciceri, R.(1997). La voce delle emozioni. Verso una semiosi della comunicazione vocale non-verbale delle emozioni. Milano: Angeli.

Armstrong, S. L., Gleitman, H., \& Gleitman, L. R. (1983). What some concepts might not be. Cognition, 13, 263-308.

Averill, J.R.(1975). A semantic atlas of emotional concepts. JSAS: Catalogue of selected Documents in Psychology, 5, 330

Barsalou, L.W. (1987). The instability of graded structure: Implications for the nature of concepts, en U. Neisser (Ed.), Concepts and conceptual development: Ecological and intellectual factors in categorisation. New York, Cambridge University Press. 
Bendix, E. H. (1966). Componential Analysis of general vocabulary: The semantic structure of a set of verbs in English, Hindi, Japanese. The Hague: Mouton

Bush, L. E. (1973). Individual differences multidimensional scaling of adjectives denoting feelings. Journal of Personality and Social Psychology, 25, 50-57.

Conte, R. H., \& Plutchik, R. (1981). A Circumplex Model for Interpersonal Personality Traits. Journal of Personality and Social Psychology, 40, 701-7II.

Church, A.T., Katigbak, M. S., Reyes, J. A. S., \& Jensen, S. M. (1998). Language and Organisation of Filipino Emotion Concepts: Comparing Emotion Concepts and Dimensions across Cultures. Cognition and Emotion, 12, 63-92.

Davitz, J. R. (1969). The language of emotions. New York: Mc Graw-Hill.

De Rivera, J.H. (1984). The structure of emotional relationships, In P.Shaver (Ed.), Review of personality and social psychology: Emotions, relationships, and health. Beverly Hills: Sage.

Duffy, E. (194I). An explanation of emotional phenomena without the use of the concept of emotion. Journal of General psychology, 25, 283-293.

D'Urso, V., \& Galati, D. (1990). Analisi dello spazio semantico di termini emozionali italiani. Ricerche di Psicologia, 14, 29-55.

Ekman, P. (1984). Expression and the nature of emotion, In K.S. Scherer \& P. Ekman (Eds.), Approches to emotion, Hillsdale, N. J., Erlbaum.

Ekman, P. (1992, a). An Argument for Basic Emotions. Cognition and Emotion. 6, 169-200.

Ekman, P. (1992, b). Facial expression of emotion: New findings, new questions. Psychological Science, 3, 34-38.

Fehr, B., \& Russell, J. A. (1984). Concept of emotion viewed from a prototype perspective. Journal of Experimental Psychology, General, II3, 464-486.

Fischer, A. (1991). Emotion Scripts. A study of social andcognitive facets of emotions. Leiden: DSWO-Press.

Fischer, A., \& Frijda, N. H. (|99|). Emotion scripts, en Emotion knowledge: Social, Cognitive, Linguistic aspects. Actes du Meeting of the European Association of Experimental Psychology près du Dipartimento di Scienze dell'Educazione dell'Università di Bari.

Frijda, N. H. (1986). The Emotions. Cambridge: Cambridge University Press.

Frijda, N. H., Zammuner, V. (1992). L'etichettamento delle proprie emozioni. Giornale Italiano di Psicologia, 19, 389-423.

Galati, D. (1986). Radicali semantici nel lessico italiano delle emozioni. Ricerche di Psicologia, 3, 5-34.

Galati, D., Massimini, F., Sini, B. (1999). II lessico delle emozioni nelle lingue neolatine: confronto tra l'italiano e il francese. Ricerche di Psicologia. 22, 57-82.

Galati, D., Sini, B. (1998a). Echelonnement multidimensionnel de termes du lexique français des émotions: une comparaison entre trois procédés d'analyse. Cahiers Internationaux de Psychologie Sociale, 37, 76-96.

Galati, D., \& Sini, B. (1998b). Les mots pour dire les émotions, recherche sur la structure du lexique émotionnel italien. Révue de Sémantique et Pragmatique, 4, 13-30.

Galati, D., Sini, B., Estaun Ferrer, S., Soler Vilageliu, O., \& Mateos, P. M. (1998). The emotional lexicon in neolatin languages. In A. Ficher, (Ed.) ISRE '98 - Proceeding of the Xth. Conference of the international Society for researche on emotion, Amsterdam: I.S.R.E Edition.

Galati, D., Tinti, C., Belghiru, L., \& Sini, B., (In press). The semantic structure of emotional lexicon of the neolatin languages: the case of Romanian. Proceedings of Isre Conference 2000. Quebec, Canada. 
Gehm, T. L., \& Scherer, K. R. (1988). Factors determining the dimensions of subjective emotional space, In K. R. Scherer (Ed.) Facets of emotion. Recent Research. Hillsdale, N.J.: Lawrence Erlbaum Associates Publishers,

Gius, E., Cozzi, A., \& Spagnotto, D. (1992). II linguaggio delle emozioni. Studio sull'organizzazione delle parole che comunicano stati emotivi. Giornale Italiano di Psicologia, 19, 563-584.

Goffman, E. (1979). Response cries, en M. von Cranach, K. Foppa, W. Lepenis, \& D. Ploog (Eds.) Human ethology Cambridge: Cambridge University Press.

Greenwald, M. K., Cook, E.W., \& Lang P. J. (1989). Affective judgement and psychophysiological response: Dimensional covariation in the evaluation of the pictorial stimuli. Journal of Psychophysiology, 3, 51-64.

Guttman, L. (1954). New approach to factor Analysis: The radex, en P. F. Razarsfeld (Ed.), Mathematical Thinking in the social sciences. Glencoe III., Free Press.

Hiatt, L. R. (1978). Classification of the emotions, en L. R. Hiatt (Ed.), Australian aboriginal concepts. Princeton, N. J: Humanities Press.

Howell, S. (1981). Rules not words, en P. Heelas, A. Lock (Eds.), Indigenous Psychologie: The antropology of the self. San Diego, Ca: Academic Press.

James, W. (I 890). The principles of Psychology, New York, Holt,

Johnson-Laird, P. N., \& Oatley, K. (1989). The language of emotions: An Analysis of a Semantic Field. Cognition and Emotion, 3, 81-123.

Keil, F.C. (1979). Semantic and conceptual development: An ontological perspective. Cambridge: Mass., Harvard University Press.

Kleinginna, R. K., \& Kleinginna, M. A. (198I). A categorized list of emotion definitions, with suggestion for a consensual definition. Motivation and Emotion, 5, 345-379.

Lang, P. J. (1984). Cognition in emotion: Concept and action, en C. E. Izard, J. Kagan, \& R. B. Zajonc (Eds.), Emotion, Cognition and Behavior. New York, Cambridge: University Press.

Lazarus, R. S., Averill, J. R. \& Opton, E. M. (1970). Toward a Cognitive theory of Emotion, In M. B. Arnold (Ed.), Feelings and Emotions. The Loyola Symposium, New York: Academic Press.

Lazarus, R. S. (1980). Emotions: a cognitive-phenomenological analysis, In R. Plutchik, \& H. Kellerman (Eds.), Emotion, I: Theories of Emotions, New York: Academic Press

Lutz, C. (1986). The domain of emotion word on Ifaluk en R. Harré (Ed.), The social construction of emotion. Oxford: Basil Blackwell Ltd..

Mandler, G. (1962). Emotion, en R. Brown, E. Galanter, E. Hess \& G. Mandler (Eds.), New directions in Psychology. New York: Holt, Rinehart, and Winston

Miller, G.A., \& Johnson-Laird, P.N. (1976). Language and Perception. Cambridge: Cambridge University Press

Neufeld, R.W. J. (1957). A multidimensional scaling analysis of schizophrenics' and normals' perception of verbal similarity. Journal of abnormal Psychology, 84, 498-507.

Neufeld, R.W. J. (1975). A multidimensional scaling analysis of schizophrenics' and normals' perceptions of verbal similarity. Journal of Abnormal Psychology, 84, 498-507

Nowlis, V, \& Nowlis, H. H. (1956). The description and anlysis of moods. Annals of the New York Academy of Science, 65, 345-355

Oatley, K., \& Johnson-Laird, P. N. (1987). Towards a cognitive theory of emotion. Cognition and Emotion, 1, 29-50.

Ortony, A., Clore, G. L., \& .Foss, M. A. (1987). The referential structure of affective lexicon. Cognitive Science, II, 34|-364. 
Osgood, C. E. (1969). On the whys and wherefores of E, P, and A.. Journal of Personality and social Psychology, 12, 194-199.

Osgood, C. E., Suci, G. J., \& Tennenbaum, P. H. (1957). The measurement of meaning, Illinois, Urbana.

Plutchik, R. (1980a). Emotion: a psychoevolutionary synthesis. New York: Harper and Row.

Plutchik, R. (1980b). A general Psychoevolutionary Theory of Emotion, In R. Plutchik, \& H. Kellerman (Eds.), Emotion. Theory, Research, and Experience. New York: Academic Press.

Plutchik, R. (|99|). The emotion. Boston: University Press of America

Poggi, I. (198I). Le interiezioni: Studio del linguaggio e analisi della mente (Interjections: Study of language and analysis of mind). Torino: Boringhieri

Poole, F. J. P. (1985). Coming into social being: Culturalimages of infants in Bimin-Kuskusmin folk psychology, In G. M. White, \& J. Kirkpatrik (Eds.), Person, Self and Experience: Exploring pacific ethnopsychologie. Barkeley: University of California Press.

Rosch, E.(1978). Principles of categorisation, en E. Rosch \& B. L. Lloyd (Eds.), Cognition and Categorisation. Hillsdale, N. J., Erlbaum.

Roget's International Thesaurus (1962) 3rd ed. New York: Thomas Y. Crowell

Russell, J. A (1978). Evidence of convergent validity on the dimensions of affect. Journal of Personality and Social Psychology, 36, II52-1 168.

Russell, J. A. (1979). Affective spaceis bipolar. Journal of personality and Social Psychology, 37, 345-356.

Russell, J. A. (1980). A circumplex Model of Affect. Journal of Personality and Social Psychology, 39, $1161-1178$.

Russell, J. A. (1983). Pancultural aspects of the human conceptual organization of emotion. Journal of Personality and Social Psychology, 45, 1281-1288.

Russell, J. A. (1987). Comments on articles by Frijda and by Conway and Bekerian. Cognition and Emotion, I, 193-197.

Russell, J. A. (1989). A Cross-cultural study of a Circumplex Model of Affect. Journal of Personality and Social Psychology, 57, 848-856.

Russell, J. A. (199|). Culture and Categorization of Emotions. Psychological Bulletin, I I0, 426$-450$

Russell, J. A., Lewicka M., Niit T. (1989). A cross-cultural study of circumplex model of affect. Journal of Personality and Social Psychology, 57, 848-856.

Russell, J. A., \& Merhabian, A. (1977). Evidence for three-factor theory of emotions. Journal of Research in Personality, II, 273-294.

Schank, R. C., \& Abelson, R. B. (1977). Scripts, plans, goals and understanding. Hillsdale: N. J. Erlbaum.

Scherer, K. R. (1984). On the Nature and Function of emotions: a component process approach, en K. R. Scherer, \& P. Ekman (Ed.), Approaches to emotion. Hillsdale: N. J., Erlbaum

Scherer, K. R.(1992). What does facial expression express? En K. Strongman (Ed.), International Review of Studies on Emotion. Chichester: Wiley.

Scherer, K. R. (1993). Neuroscience projections to current debates in emotion psychology. Cognition and Emotion, 7, I-4I.

Scherer, K. R. (1994). Affect Bursts, en S.H.M. van Gooze, N.E. van de Poll, \& J.A. Sergeant (Eds), Emotions: Essays on Emotion Theory. Hillsdale, NJ: Erlbaum.

Schlosberg, H. (1952). The description of facial expressions in terms of two dimensions. Journal of Experimental Psychology, 44, 229, 37. 
Schlosberg, H. (1954). Three dimensions of emotion. Psychological Review, 6I, 81-88.

Shaver, P., Schwartz, J., Kirson, D.,\& O'Connor, C. (1987). Emotional knowledge: Further exploration of a prototype approach. Journal of Personality and Social Psychology, 52, 1061$-1086$

Stein, N. L., \& Trabasso, T. (1992). The organisation of emotional experience: Creating links among Emotion, Thinking, Language, and intentional action. Cognition and emotion, 6, 225-244 .

Stone, L. A., \& Coles, G. J. (1970). Correlational similarity: The basis for a new revised method of similarity analysis. Studia Psychologica (Bratislava), 12, 258-265.

Trentin, R. (1988). Emozioni e processi cognitivi, In V. D'Urso \& R. Trentin (Eds.), Psicologia delle emozioni. Bologna: II Mulino.

Van Gooze, S., \& Frijda, N. H. ( 1 993). Emotion words used in six European Conutries. European Journal of Social Psychology, 23, 89-95

Watson, J. B., \& Rayner, R. (1920). Conditioned emotional reactions. Journal of Experimental Psychology, 3, I-14.

Watson, J. B., \& Tellegen, A. (1985). Toward a consensual structure of mood, Psychological Bulletin, 95, 219-235

Wierzbicka, A. (1992). Talking about emotions: Semantics, Culture, and Cognition, Cognition and Emotion, 6, 285-319.

Wundt, W. (1890). Grundriss der Psychologie. Leipzig, Engelman.

Zajonc, R.B. (1980). Feeling and thinking: Preferences need no inferences. American Psychologist, $35,|5|-175$. 


\section{Bernard Rimé}

Université de Louvain,

Louvain-la-Neuve, Belgique

\section{ÉMOTION ET MÉMOIRE: \\ LA RÉMANENCE DES EXPÉRIENCES ÉMOTIONNELLES}

\section{INTRODUCTION}

Dans la littérature scientifique, les émotions sont surtout étudiées sous deux angles principaux. II s'agit d'une part des conditions de leur déclenchement, et d'autre part, des composants de l'expérience émotionnelle. Dans le premier cas, on examine des variables telles que les circonstances extérieures, l'évaluation cognitive qu'elles suscitent, la modulation de celle-ci par des facteurs de contexte, etc. Dans le second cas, on porte l'attention sur les réponses dans les différents systèmes: manifestations faciales, physiologiques, neuropsychologiques, comportementales, subjectives, et cognitives. Le chapitre qui suit sera consacré à une question qui se situe complètement en aval des précédentes. Nous allons examiner la rémanence des expériences émotionnelles.

Selon la définition qu'en donne le dictionnaire Robert, le terme rémanence signifie «permanence» ou «persistance». En physique, il désigne la persistance partielle de l'aimantation après le retrait de l'influence magnétique. Le même dictionnaire indique que ce terme est également utilisé dans le domaine de la perception visuelle. La technique du cinéma est fondée sur la rémanence des images visuelles. En étendant ici la notion de rémanence au domaine de l'émotion, nous viserons donc l'impact conservé par les expériences émotionnelles sur les différents niveaux du fonctionnement du sujet après la fin de l'épisode qui a provoqué l'émotion. De quoi s'agit-il?

De nos jours, tout le monde sait que des expériences émotionnelles à caractère traumatique peuvent avoir un impact prolongé. En particulier, l'exposition soudaine à la mort ou à la mutilation peut entraîner, et parfois pour longtemps, le revécu de l'événement sous forme d'irruption d'images, d'impression soudaine que l'événement se reproduit, de rêves ou de 
cauchemars, ou de réactions physiologiques et subjectives de stress et détresse lorsque quelque chose rappelle l'événement. Ce sont des manifestations de rémanence de l'émotion puisqu'il s'agit de l'impact d'un événement émotionnel passé sur le fonctionnement actuel de l'individu. Si la rémanence post traumatique nous est familière aujourd'hui, l'histoire de la santé mentale est là pour nous rappeler qu'il n'en a pas toujours été ainsi. L'idée qu'un événement émotionnel puisse affecter ultérieurement le fonctionnement cognitif et affectif a fait pendant longtemps l'objet de résistances importantes dans les milieux de la psychopathologie (Van der Kolk, Weisaeth, \& Van der Hart, 1996). Ces résistances n'ont été vaincues que par l'observation massive de séquelles psychologiques chez les militaires américains après la guerre du Vietnam (Kulka, Schlenger, Fairbank, Hough, Jourdan, Marmar \& Weiss, 1990). II y a à peine une vingtaine d'années que le syndrome de stress post traumatique qui décrit de telles manifestations est entré dans le Diagnostic and Statistical Manual of Mental Disorders (DSM) (American Psychiatric Association, 1980). Depuis lors, ce syndrome est l'objet d'étude privilégié d'une spécialité scientifique que l'on désigne parfois comme la «psychotraumatologie».

Curieusement, les aléas du découpage des spécialités scientifiques entretiennent une ligne de démarcation entre la psychotraumatologie d'une part et la psychologie des émotions d'autre part. Celle-ci étudie les expériences émotionnelles de la vie courante comme la joie, la colère, la tristesse, la peur, la honte, etc. (voir par exemple Frijda, 1986; Oatley \& Jenkins, 1996; Rimé \& Scherer, 1990). Depuis les travaux de Cannon (1929), on y aborde les émotions dans une perspective homéostatique. Selon ce point de vue, l'émotion est déclenchée par une situation qui requiert une réponse urgente. Elle met en oeuvre des fonctions psychophysiologiques qui doivent permettre à l'individu de pallier la situation urgente. Elle conduit ainsi au rétablissement dans l'organisme de l'état qui était le sien avant la crise. Dans une telle logique, l'émotion s'achève avec la fin de l'épisode émotionnel. L'intérêt ne se porte pas au-delà de ce terme, et on n'envisage donc pas qu'il puisse y avoir une rémanence de l'émotion. Cependant, des travaux que nous avons menés au cours de la dernière décennie montrent que la rémanence des expériences émotionnelles de la vie courante est une réalité. Trois types de manifestations ont été observés à cet égard: l'effet de réactivation, la rumination mentale, et la partage social des expériences émotionnelles. Nous allons examiner brièvement chacune d'elles 


\section{TROIS TYPES DE MANIFESTATIONS DE LA RÉMANENCE DES ÉMOTIONS}

L'effet de réactivation. Dans l'une de nos études, nous avons invité les participants à se remémorer un épisode émotionnel de leur passé récent (Rimé, Noël, \& Philippot, 199/a). Les instructions précisaient le type d'émotion selon quatre conditions différentes. Certains devaient se rappeler un épisode au cours duquel ils avaient éprouvé de la peur, d'autres de la tristesse, d'autres de la joie, d'autres de la colère. Tous devaient ensuite rédiger un texte dans lequel ils décrivaient en grand détail cet épisode. Enfin, ils ont répondu à un questionnaire examinant leur expérience pendant qu'ils écrivaient. Quel que fut le type d'émotion réévoquée, on constatait que le rappel avait activé chez presque tous ( 1 ) des images mentales vives de l'épisode, (2) des changements corporels émotionnels intenses, et (3) une expérience subjective semblable à éprouvée au cours de l'épisode initial. Un simple rappel permet donc d'observer l'impact d'une expérience émotionnelle passée sur le fonctionnement cognitif et affectif actuel.

La rumination mentale. L'induction d'un rappel n'est pas indispensable pour observer la rémanence des expériences émotionnelles. Nos études ont montré que les expériences émotionnelles se représentent spontanément en mémoire après coup (Rimé, Mesquita, Philippot, \& Boca, 199/b; Rimé, Philippot, Boca, \& Mesquita, 1992). Une «rumination mentale» de l'expérience émotionnelle se manifeste sous forme d'images, de pensées, ou de dialogues intérieurs. Qu'il s'agisse de joie, de colère, de tristesse, de peur, de honte ou d'une autre émotion, la rumination mentale est pratiquement toujours présente après l'émotion. Ses manifestations sont généralement répétitives. On observe une corrélation positive modérée entre la fréquence de la rumination mentale et l'intensité de l'émotion vécue dans l'épisode source. Les expériences émotionnelles de forte intensité peuvent susciter une rumination mentale qui prend une allure obsédante. II s'agit bien de manifestations de rémanence de l'expérience émotionnelle, puisque l'épisode émotionnel affecte le fonctionnement de la personne en un temps qui est largement ultérieur.

Le partage social. La rémanence de l'émotion ne se limite pas à des manifestations internes à la personne. On l'observe également sous forme externe et interpersonnelle, dans une propension à verbaliser l'expérience émotionnelle, et à en reparler avec l'entourage. C'est le «partage social de l'émotion», qui se présente après l'émotion dans 80 à $95 \%$ des cas (Rimé \& al., 199 |b; Rimé \& al., 1992; Rimé, Finkenauer, Luminet, Zech, \& Philippot, 
1998). II apparaît le plus souvent dès le jour où l'événement s'est produit et il est généralement répétitif. Les cibles sont principalement les intimes. Plus l'expérience émotionnelle a été intense, plus le partage social sera répétitif, de sorte que les épisodes d'intensité élevée sont généralement partagés à de nombreuses reprises. On rencontre le partage social de l'émotion quel que soit le type d'émotion impliquée dans l'expérience. Toutefois, la honte et la culpabilité sont associées à des taux plus réduits de communication. Elles sont également associées aux expériences émotionnelles gardées secrètes (Finkenauer \& Rimé, 1998). Egalement présentes chez les hommes et les femmes, les manifestations du partage social de l'émotion s'observent déjà chez les enfants, elles apparaissent ensuite de manière comparable dans toutes les classes d'âges, et leur évolution au troisième âge (65-95 ans) se fait dans le sens d'une accentuation. Enfin, le partage social de l'émotion a été observé de manière globalement comparable dans diverses populations européennes, nord-américaines et asiatiques (Corée, Japon, Singapour, Indes) (Mesquita, 1993; Rimé, Yogo, \& Pennebaker, 1996; Singh-Manoux, 1998; Singh-Manoux \& Finkenauer, 200I;Yogo \& Onoué, 2000). C'est donc un phénomène d'une très grande généralité.

Conclusions. Ces données montrent que les événements émotionnels de la vie courante suscitent des manifestations de rémanence, comme c'est le cas pour les événements émotionnels traumatiques. Même si dans le second cas, la rémanence peut parfois handicaper gravement le fonctionnement de l'individu, on peut conclure que les deux types d'expérience sont sur ce plan dans un rapport de continuité. Nous avons déjà discuté ailleurs la portée scientifique d'une telle constatation (Philippot \& Rimé, 1998). Nous avons également passé en revue à diverses reprises le détail de nos recherches sur la rumination mentale et sur le partage social de l'émotion

82 (Rimé \& al., 1992; Rimé \& al., 1998, Rimé, Corsini, \& Herbette, 2002; Rimé \& Herbette, sous presse;). Dans ce chapitre, nous voudrions tenter de systématiser les informations relatives à cette dynamique particulière qui caractérise les souvenirs d'expériences émotionnelles. Nous examinerons d'abord les phases de l'évolution du souvenir de l'expérience émotionnelle. Nous chercherons ensuite à préciser en quoi consiste la mémoire d'une expérience émotionnelle. Enfin, nous aborderons la question des facteurs qui est la question la plus critique pour la compréhension de la rémanence des expériences émotionnelles. 


\section{LES PHASES DE L'ÉVOLUTION D'UN SOUVENIR ÉMOTIONNEL}

De la rémanence initiale au souvenir dormant. Immédiatement après l'épisode, les expériences émotionnelles se représentent en mémoire de travail et suscitent donc une activité cognitive sous forme de rumination mentale ainsi qu'une activité sociale sous forme de partage social. En particulier, l'expérience émotionnelle et les circonstances qui l'ont induite sont communiquées, décrites, commentées, et discutées. Dans ces moments, les composantes de l'univers phénoménal de cette expérience tendent à envahir à nouveau la conscience du sujet. A mesure que le temps passe, qu'il s'agisse de la rumination mentale ou du partage social, les manifestations de la rémanence déclinent et l'impact de l'expérience émotionnelle sur le fonctionnement courant se réduit peu à peu jusqu'à devenir négligeable. Par exemple, une semaine après un examen universitaire important, 100\% des étudiants suivis en avaient reparlé au cours des 48 heures précédant l'enquête; deux semaines après l'examen, un taux de $94 \%$ était observé, et trois semaines après l'examen, ce taux était tombé à 50\% (Rimé \& al., 1998). Mais la pente du déclin progressif est fonction de l'intensité initiale de l'expérience. Plus l'émotion d'origine a été intense, moins cette pente sera raide. Ainsi, dix jours après le décès d'un proche, $97 \%$ des répondants avaient reparlé de leur expérience émotionnelle dans les 48 heures précédant l'enquête; quatre semaines après le décès, le taux était de $86 \%$, et 12 semaines après l'événement, il s'élevait encore à 79\% (Zech, 1992). Selon le cas, la pente de déclin pourra donc se développer sur une échelle qui s'inscrira en heures, en jours, en semaines, en mois, voire en années. Tait \& Silver (1989) ont étudié chez leurs répondants l'impact actuel du «pire événement de leur vie». Bien que les événements ainsi récoltés aient eu lieu en moyenne 22,8 années auparavant, on observait qu'ils donnaient encore lieu à des rémanences chez près de la moitié des participants. Par contre, les autres répondants avaient atteint la phase finale d'évolution de la rémanence pour cet événement. C'est la phase «dormante» du souvenir qui se caractérise par l'absence de tout impact sur le fonctionnement actuel de la personne. Nous adoptons ce terme parce que nous verrons plus loin qu'il y a lieu de penser que le souvenir d'une expérience émotionnelle ne s'éteint jamais. 
Expériences émotionnelles non résolues. Certaines souvenirs d'expériences émotionnelles n'évoluent pas cependant vers l'état dormant. Quand cela se présente, c'est problématique. En effet, si un souvenir continue à mobiliser les ressources attentionnelles, il le fait au détriment des besoins de l'adaptation aux situations nouvelles. On parlera dans ce cas d'expérience émotionnelle non résolue. Trois éléments les caractérisent. En premier lieu, les réitérations mentales qu'elles suscitent gênent la vie quotidienne et le besoin de reparler de l'expérience perpétue. Toutefois, du fait de la saturation de l'entourage, le partage social effectif est réduit, voire absent. Mais il s'instaure à la moindre occasion. En deuxième lieu, les émotions réactivées lors de chaque rappel demeurent d'intensité élevée. En troisième lieu, la personne a conscience du statut particulier de cette expérience. Elle reconnaît son caractère non résolu et exprime le sentiment de «porter» encore cet épisode au quotidien ou «de ne pas être en paix» à son sujet (Tait \& Silver, 1989).

Des instructions simples permettent de montrer ces différentes caractéristiques. Nous avons demandé à des étudiants de se rappeler puis de décrire une expérience émotionnelle, selon deux types d'instructions (Rimé, Hayward, \& Pennebaker, 1996). Ils devaient se rappeler soit d'une expérience non résolue (on spécifiait simplement qu'ils devaient encore être affectés actuellement par cette expérience), soit d'une expérience résolue, (elle ne les affectait plus). Après le rappel, tous ont répondu à un questionnaire examinant l'impact que l'expérience avait eu au moment où elle s'était produite et celui qu'elle conservait dans la vie actuelle. Les épisodes évoqués dans les deux cas ne se distinguaient ni pour l'intensité de leur impact émotionnel à l'origine, ni pour les manifestations de la rémanence initiale. Dans les deux conditions, le partage social avait été initié aussi rapidement après l'épisode et il avait été répété avec une fréquence 84 comparable. Par contre, les expériences résolues et non résolues différaient très significativement pour tous les indicateurs de rémanence actuelle. En particulier, pour les épisodes non résolus, l'intensité des émotions éprouvées pendant le rappel expérimental était supérieure, le besoin actuel de reparler de l'épisode était plus intense, et le partage social effectif au cours des semaines précédant l'étude était plus fréquent que pour les épisodes résolus. Ceci suggère que les gens peuvent distinguer dans leurs souvenirs autobiographiques les expérience émotionnelles non résolues et les expériences émotionnelles résolues. 
Expériences émotionnelles réactivées. Nous avons vu plus haut que lorsqu'on induisait le rappel induit d'une expérience émotionnelle, une réactivation de cette expérience s'ensuivait (Rimé \& al., 199la). C'est également ce qui se passe dans la vie courante lorsque des conditions de rappel sont rencontrées. L'événement passé retrouve alors un impact actuel et l'expérience phénoménale qui avait caractérisé cet événement se reconstitue jusque dans ses prolongements physiologiques. Si les éléments appropriés de rappel sont rencontrés, des expériences émotionnelles très anciennes peuvent être réactivés avec une force considérable. Dans «A la recherche du temps perdu», Marcel Proust a décrit le flot des sentiments, sensations et autres souvenirs d'enfance qui étaient ravivés par la simple ingestion d'une madeleine. II a offert du même coup une belle illustration littéraire de la réactivation, révélant ainsi la résistance à l'extinction des souvenirs chargés d'émotion.

Conclusions. Les manifestations de rémanence sous forme de rumination mentale et de partage social sont des phénomènes normaux au cours de la période qui fait suite à l'émotion. Au vu de la généralité de ces manifestations, on a toutes les raisons de penser qu'elles font partie intégrante de l'émotion. Leur destin normal est ensuite le déclin progressif, et l'issue attendue est l'état de souvenir dormant. Le meilleur prédicteur connu de la pente de cette évolution est l'intensité de l'émotion vécue à l'origine. Certaines expériences émotionnelles font exception au principe du déclin et continuent à mobiliser l'attention au détriment de l'adaptation courante. On ne dispose pas encore de prédicteur clair de ces expériences non résolues. Enfin, il semble bien que tout souvenir émotionnel dormant puisse être réactivé lorsque des éléments de rappel sont rencontrés. Beaucoup de questions se posent maintenant. Pourquoi les expériences émotionnelles ont-elles cette particularité de susciter la rémanence? Pourquoi suscitent-elles d'autant plus de rémanence que l'intensité de l'émotion a été élevée? Pourquoi les manifestations de la rémanence déclinent-elles à mesure que le temps s'écoule? Pourquoi ces manifestations se perpétuent-elles dans certains cas? Pour aborder ces questions, il est nécessaire de rechercher les particularités des expériences émotionnelles à deux niveaux différents: (I) au niveau des éléments qui constituent leur représentation en mémoire à long terme, et (2) au niveau des facteurs susceptibles de les rappeler en mémoire de travail. 


\section{REPRÉSENTATION EN MÉMOIRE À LONG TERME}

Eléments de l'expérience. Une expérience émotionnelle se distingue de l'expérience ordinaire par la masse des éléments qui y entrent en ligne de compte, et ce à trois niveaux différents de l'univers expérientiel. Le tableau I propose une liste de synthèse de ces éléments établie à partir de Lang (1993) et de Scherer (1984). Le premier niveau est celui du donné de la situation. Une situation émotionnelle a généralement la particularité de «déborder» l'individu. Le donné externe à percevoir et à enregistrer se présente souvent de manière surabondante dans un empan temporel réduit. II touche toutes les modalités d'entrées, auditives, visuelles, olfactives, tactiles, cutanées, vestibulaires et kinesthésiques. Le deuxième niveau est celui des données issues du travail cognitif automatique et du travail cognitif contrôlé qui se développent dans ces conditions. En situation émotionnelle, le potentiel cognitif d'appréciation des situations doit répondre à des sollicitations multiples dans des délais extrêmement brefs. Le processus se déroule de manière séquentielle (Scherer, 1984) et il engendre un ensemble vaste de significations diverses, parfois contradictoires. Le troisième niveau des données qui caractérisent les expériences émotionnelles est celui des réponses. L'émotion sollicite l'ensemble psychophysiologique que constitue l'individu en mettant en jeu tous les systèmes de réponses dont celui-ci dispose, et ce à un point qu'aucune situation non émotionnelle n'approche. Toute expérience, qu'elle soit émotionnelle ou non émotionnelle, comporte des éléments des trois niveaux que nous venons de parcourir. Cependant, il existe une relation de fait entre l'intensité de l'émotion qui caractérise une expérience et l'abondance des éléments aux différents niveaux. Cette remarque s'applique particulièrement aux éléments psychophysiologiques. Au plus intense est l'émotion, au plus dense sera l'ensemble psychophysiologique qui constitue l'expérience.

II faut conclure de ce qui précède qu'une expérience émotionnelle propose au processus de mise en mémoire un matériel plus abondant et plus diversifié qu'une expérience ordinaire. C'est sans doute un aspect à prendre en considération si on veut comprendre pourquoi les expériences émotionnelles suscitent la rémanence.

Les émotions dans la mémoire à long terme. La littérature n'aborde guère la question de la manière dont le souvenir d'un épisode émotionnel donné se constitue dans la mémoire à long terme. Mais certaines théories de 


\section{TABLEAU I LES ÉLÉMENTS DE L’EXPÉRIENCE ÉMOTIONNELLE}

NIVEAU I - Données externes saisies dans les différentes modalités d'entrées (auditives, visuelles, olfactives, tactiles, cutanées, vestibulaires et kinesthésiques), notamment (Lang, 1993):

- les détails physiques de l'objet ou de la situation

- les modifications de la configuration de l'objet

- les mouvements de l'objet (par exemple, approche, évitement...)

- l'espace physique, les lieux et la localisation générale

- la présence ou l'absence des autres comme observateurs ou participants

- les commentaires faits par les autres

- la douleur éventuelle, sa localisation corporelle, ses caractéristiques

NIVEAU 2 - Données issues de l'évaluation cognitive séquentielle de la situation, notamment (Scherer, 1986):

- de la nouveauté de la situation,

- de ses conséquences hédonistiques,

- de sa signification pour les buts poursuivis par le sujet,

- de la capacité que celui-ci possède d'y faire face,

- de la compatibilité de l'événement avec les normes du sujet.

NIVEAU 3 - Données internes issues des réponses dans les différents systèmes, notamment (Lang, 1993):

- les signaux vocaux

- les verbalisations explicites ou implicites

- les manifestations somatomotrices diverses (tension musculaire, manifestations expressives, manifestations motrices incontrôlées, manifestations motrices organisées)

- les manifestations viscérales aux différents niveaux du système nerveux autonome (sous-système cardiaque, cutané, vasculaire, pilomoteur, salivaire, respiratoire, gastro-intestinal, urinaire)

- les manifestations qui caractérisent le fonctionnement cognitif (confusion, vivacité, distorsion, perte du contrôle de la pensée, désorientation spatio-temporelle)

- les manifestations liées à l'adaptation des organes sensoriels (changements posturaux, mouvements oculaires, mouvements de la tête). 
l'émotion se fondent sur lidée que la mémoire à long terme cumule sous forme de réseaux associatifs schématiques ou prototypiques, les informations issues d'expériences émotionnelles similaires (Lang, 1979; Leventhal, 1984). L'amorçage de tels réseaux serait à la base du processus de déclenchement de l'émotion. On peut s'inspirer de ces théories pour se faire une idée de la manière dont la mémoire à long terme emmagasine les souvenirs de chaque épisode émotionnel particulier. Cela conduit à considérer (voir en particulier Lang, 1979, 1993) qu'un réseau associatif spécifique rassemblerait l'ensemble des éléments qui ont coexisté pendant une expérience émotionnelle donnée. Ce réseau lierait ainsi les données de la situation, celles des significations engendrées par le traitement cognitif de cette situation, et celles des réponses de l'ensemble psychophysiologique. II comporterait en outre des adresses fonctionnelles de sortie vers les efférences physiologiques et vers l'action manifeste. Une fois constitué, ce réseau associatif devient le substrat mnémonique de cet épisode émotionnel qu'il représente désormais dans la mémoire à long terme.

Selon les conceptions classiques de la mémoire, pour qu'une expérience passée retrouve un impact sur l'adaptation actuelle, son souvenir doit être ramené en mémoire de travail par des facteurs de rappel. Les manifestations de la rémanence doivent être comprises dans cette perspective. Elles s'expliquent nécessairement par la présence dans le contexte actuel, d'éléments qui s'apparient avec des composants du réseau mnémonique de cette expérience. Nous terminerons donc ce chapitre par l'examen de la question des facteurs de rappel.

\section{FACTEURS DE RAPPEL}

Eléments d'appariement. Le souvenir d'une expérience émotionnelle donnée sera réactivé si des éléments situationnels ressemblant à ceux que comporte le réseau mnémonique de cette expérience se représentent dans le contexte actuel. II le sera également si des réponses psychophysiologiques du type de celles qui ont été emmagasinées dans la situation d'origine, sont réactivées. De même, le souvenir sera réactivé si des significations semblables à celles qui furent enregistrées au cours de l'expérience d'origine sont évoquées à nouveau. Le substrat mnémonique d'un épisode émotionnel particulier sera donc réactivé chaque fois que le contexte actuel, externe 
ou interne, comportera des éléments qui s'apparient avec des éléments associés à ce substrat. Les différents éléments qui ont été envisagés au tableau | constituent donc autant de voies d'accès potentielles. II suffit que le contexte actuel les remette en jeu. Lorsque cet appariement se présente, l'activation se produit et se diffuse ensuite dans l'ensemble du réseau. Au meilleur l'appariement, au plus forte sera l'activation. Si l'activation est suffisamment importante, elle se diffusera jusqu'aux adresses de sortie, entraînant ainsi la mise en oeuvre effective des changements physiologiques.

Pour les épisodes émotionnels, la gamme des facteurs d'appariement est donc très étendue, et en tout cas beaucoup plus étendue que pour la plupart des expériences non émotionnelles. Cela explique en partie pourquoi les expériences émotionnelles sont sujettes aux manifestations de rémanence. Toutefois, dans le cas des expériences émotionnelles, la gamme des facteurs de rappel ne s'arrête pas aux éléments d'appariement. II faut ajouter à cette gamme déjà très étendue, des facteurs de rappel qui leur sont propres.

Les séquelles de l'épisode. Les expériences émotionnelles laissent généralement des marques dans différents registres de la vie courante du sujet. Ainsi, dans le registre matériel, les épisodes émotionnels laissent souvent des traces dans les biens ou les possessions des acteurs, qu'il s'agisse de dommages à la propriété, de dégâts à la voiture, ou d'autres pertes ou dégradations d'objets. Dans le registre du corporel, l'épisode peut se solder par des blessures, des lésions, ou des handicaps divers qui affectent le sujet lui-même ou des personnes dont il a le souci. Dans le registre économique, l'épisode peut entraîner des coûts ou des pertes financières qui peuvent altérer la vie quotidienne. Dans le registre social, l'épisode peut déboucher sur des conflits, des litiges, des mécontentements, des ruptures, des procédures juridiques ou judiciaires. II s'agit dans chacun de ces cas de séquelles de l'épisode émotionnel, et donc d'indices concrets que l'épisode émotionnel laisse dans la vie courante de la personne. De tels indices forment des facteurs de rappel particulièrement efficaces. Ils peuvent donc contribuer dans une mesure importante à la rémanence. Ainsi, dans une étude récente, on a examiné à la fois les séquelles et la rémanence résultant d'épisodes émotionnels importants chez des répondants adultes (Rimé \& Gérard, 200I). Ces répondants ont indiqué l'importance des séquelles physiques, matérielles, économiques, et sociales laissées par l'épisode dans 
leur vie actuelle. Ils ont également répondu à une échelle évaluant la rémanence actuelle de cet épisode. L'examen des relations entre ces variables montrait un $r=-.37(p<.05)$ entre l'importance des séquelles actuelles et le degré estimé de résolution. En outre, une corrélation de. 57 $(p<.001)$ apparaissait entre l'importance des séquelles actuelles et la fréquence actuelle du partage social. Ces relations sont donc tout à fait cohérentes avec le principe selon lequel les séquelles activent la rémanence.

Aux différents types de séquelles que nous avons envisagées ci-dessus, il faut ajouter une catégorie supplémentaire. Parmi les séquelles laissées chez l'individu par une expérience émotionnelle, celles qui concernent le domaine cognitif sont particulièrement cruciales pour la compréhension de la rémanence des émotions.

Les séquelles cognitives. De toutes les séquelles susceptibles de résulter de l'expérience émotionnelle, les séquelles cognitives sont à la fois les moins visibles et les plus influentes. II est fréquent qu'alors que l'épisode émotionnel ne laisse plus aucune autre séquelle (matérielle, physique, économique, ou sociale...) dans la vie actuelle, d'importantes manifestations de rémanence se perpétuent sans que la personne puisse en déceler la source. En fait, les expériences émotionnelles mettent en jeu des éléments que le sens commun ignore complètement. Elles sont souvent suivies de séquelles cognitives qui constituent de puissants facteurs de rappel. Nous allons évoquer succinctement la manière dont elles se développent.

L'univers physique et social dans lequel évoluent les êtres humains comporte une part importante d'imprévus, de hasards, d'accidents, de risques, de malveillances et d'injustices. Pour leur part, les êtres humains ont développé un sens aigu de leur bien-être, de leur intégrité physique et de leur survie. Evoluer au quotidien avec une conscience claire des menaces auxquelles la vie les expose éveillerait chez eux des niveaux d'anxiété 90 propres à rendre l'action et l'adaptation impossibles. C'est donc au sein d'un univers modélisé cognitivement qu'ils déploient leurs activités ordinaires. De nombreuses observations empiriques ont documenté les théories implicites au moyen desquelles les individus constituent cet espace vital rassurant. Ainsi, par exemple, ils utilisent un arsenal de protection contre l'idée de leur propre mortalité (Solomon, Greenberg, \& Pyszcsynski, 1991); ils envisagent les situations comme si leur propre personne bénéficiait d'une certaine invulnérabilité (Perloff, 1983); ils se figurent leur propre destinée sous l'angle d'un optimisme non réaliste (Taylor \& Brown, 1988; Taylor, Kemeny, Reed, 
Bower, \& Gruenewald, 2000; Weinstein, 1980; Weinstein \& Klein, 1996); ils déploient d'importants efforts mentaux en vue de maintenir une vision selon laquelle le monde est juste (Lerner, 1980) et bienveillant (Janoff-Bulman, 1992); ils surestiment leur capacité de contrôler les événements (Langer, 1975); ils endossent largement les propositions culturelles selon lesquelles le monde dans lequel ils vivent a un sens (Berger \& Luckmann, 1966//996). En somme, les êtres humains développent des représentations cognitives biaisées de la réalité physique, de la réalité sociale, et d'eux-mêmes. Ils entretiennent continuellement une série de constructions cognitives qui, prises ensemble, forment une enveloppe symbolique capable de voiler les menaces auxquelles la vie les expose, et de les doter de sentiments de cohérence, de maîtrise, de stabilité et de sécurité. Ces constructions ne sont pas conscientes, puisque c'est précisément leur fonction que d'être confondues avec le réel.

Les expériences émotionnelles ont systématiquement la particularité d'ébranler voire même d'infirmer chez le sujet, ces théories implicites sur le monde, les autres et soi-même sur lesquelles il s'appuie. N'importe quel exemple d'épisode émotionnel permet de l'illustrer. Ainsi, si un conducteur se comporte de manière inconsidérée sur la route mettant brusquement en cause mon intégrité physique, mon appréhension rapide et automatique du danger imminent se soldera par une soudaine frayeur. C'est là qu'on arrête généralement l'analyse des liaisons cognition-émotion qui caractérisent les événements émotionnels. Mais en réalité, l'impact d'un tel événement se prolongera bien au-delà de la seule appréhension du danger. II affectera directement une partie de l'échafaudage des théories implicites sur lesquelles je me repose habituellement afin de pouvoir emprunter la route sans angoisse. Ces théories me permettent en effet de croire que je suis à l'abri du danger, que je bénéficie d'une invulnérabilité relative, que mon destin est plus favorable que celui de la plupart des autres gens, que je suis un conducteur plus habile que les autres, que les autres conducteurs sont maîtres d'eux, qu'ils se comportent de manière raisonnable, que ma voiture est plus robuste que la moyenne des autres voitures, etc. Au moment où je fais l'expérience de l'accident imminent, chacune de ces constructions se trouvera ébranlée. Si l'accident a lieu, leur mise en question risque bien d'atteindre un point critique. S'il s'ensuit des dommages graves pour mes biens et ma personne, l'infirmation de ces constructions pourrait devenir irréversible pour une longue période. 
Dans tous ces cas, les éléments de l'expérience émotionnelle se révèlent en relation d'incompatibilité avec des constructions cognitives qui font partie de l'expérience courante. C'est donc une situation de dissonance cognitive qui s'installe (Festinger, 1957). Tant qu'elle subsistera, la dissonance aura l'effet d'un facteur de rappel. Elle rappellera le souvenir de l'épisode émotionnel en mémoire active, empêchant ainsi son accession au statut de souvenir dormant dans la mémoire à long terme. Horowitz (1976) a décrit le développement d'un processus de ce type dans le cas des épisodes émotionnels à caractère traumatique. II a notamment montré comment cette dynamique cognitive entraînait la perpétuation des manifestations du syndrome de stress post traumatique. Notre propos est ici d'étendre la prise en considération de cette dynamique à toute expérience émotionnelle, y compris celles de la vie la plus courante. Elle contribue généralement dans une mesure importante aux différentes manifestations de rémanence qui font suite aux épisodes émotionnels.

\section{CONCLUSIONS GÉNÉRALES}

Dans ces pages, nous avons montré que les expériences émotionnelles de la vie courante se prolongeaient au-delà de l'événement par des manifestations de rémanence. Nous avons décrit les différentes formes que pouvaient prendre la rémanence des expériences émotionnelles, dans la réactivation par le rappel, la rumination mentale et le partage social. Nous avons examiné les différentes phases de l'évolution de la rémanence, avec la phase initiale, le déclin progressif, et la phase du souvenir dormant. Nous avons constaté que certaines expériences émotionnelles n'atteignaient pas cette dernière phase et suscitaient la rémanence de manière chronique. Nous avons

92 également vu que les souvenirs dormants pouvaient être réactivés. Nous avons examiné les particularités de la mémorisation pour constater que l'expérience émotionnelle se caractérise par l'abondance des éléments à emmagasiner. Enfin, nous avons détaillé les différents types de facteurs de rappel à l'œuvre dans la réactivation des expériences émotionnelles: éléments d'appariement, séquelles diverses, et séquelles cognitives.

La masse des facteurs de rappel permet de rendre compte de la rémanence qui caractérise les expériences émotionnelles. Immédiatement après l'épisode, les facteurs de rappel se retrouvent généralement en 
abondance dans le contexte immédiat de la personne. Par la suite, ces éléments s'estompent progressivement et laissent dans le foyer attentionnel, la place à de nouvelles situations et à de nouveaux problèmes. Le déclin de la rémanence en résulte. C'est sans doute ce type d'évolution qui a donné lieu au proverbe qui dit que «le temps efface toutes les blessures». Ce processus connaît des exceptions. Pour éclairer les expériences émotionnelles qui demeurent non résolues, il faut faire prendre en considération les différents éléments de la gamme des facteurs de rappel.

Une question clé dans cette problématique de la rémanence est celle de la résistance à l'extinction qui caractérise les souvenirs d'expériences émotionnelles. Elle apparaît à première vue comme une propriété contre-adaptative. Pourquoi l'individu ne peut-il pas se débarrasser des souvenirs émotionnels anciens? Pourquoi semble-t-il devoir demeurer assujetti au risque du rappel de ces souvenirs, et donc, à cette forme de tyrannie du passé que constitue la rémanence de l'expérience émotionnelle? La réponse suivante pourrait être proposée. Les événements qui suscitent les émotions sont plus importants que les autres pour le bien-être, l'intégrité physique ou la survie. En mémorisant les caractéristiques de ces événements, l'individu se forge des outils précieux d'anticipation. Quand il dispose de ces outils, il est à l'abri du dépourvu car lorsque le contexte présente des indices similaires à ceux de l'épisode émotionnel, l'activation du souvenir est alors immédiate. L'expérience antérieure assure la mise à disposition instantanée de l'information pertinente: séquence de l'événement, caractéristiques des éléments de la séquence, données d'appréciation cognitive, réponses appropriées, réponses inappropriées, etc. L'afflux des souvenirs réduit donc la prise au dépourvu, et augmente d'autant les capacités adaptatives et le sentiment de contrôle. C'est le bénéfice que retire chaque individu de son expérience de vie. II n'y a ici rien d'autre qu'une manifestation spécifique des principes classiques de l'apprentissage. Mais l'observation de la rémanence des souvenirs émotionnels donne à penser que ces principes opèrent de façon particulièrement puissante quand il y a émotion.

Par la voie des processus associatifs, chaque épisode émotionnel est donc une nouvelle occasion d'étendre le savoir sur les aléas du monde dans lequel on vit, et sur la manière d'affronter ces aléas. Le processus de la rémanence lui-même, avec ce qu'il implique de traitement cognitif et de traitement social de l'information liée à ces situations, pourrait bien être en réalité un outil ex post facto dont la fonction serait d'accroitre encore ce savoir par 
I'utilisation des voies conceptuelles et du dialogue, avec notamment la spéculation, la délibération et le consensus social.

Dans la perspective qui vient d'être développée, on peut donc comprendre pourquoi l'information emmagasinée en mémoire à long terme à propos d'un épisode émotionnel semble devoir échapper à l'extinction. Elle représente le fruit de l'expérience des situations critiques qui ont été traversées. II serait contre-adaptatif qu'une extinction progressive des liens associatifs qui constituent cette information mnémonique puisse priver l'individu de ce fruit.

\section{RÉFÉRENCES}

American Psychiatric Association (1980). Diagnostic and Statistical Manual of Mental Disorders, Third Edition. Washington DC:: American Psychiatric Association.

Berger, P. \& Luckmann, T. (1996). La construction sociale de la réalité (Traduction de l'ouvrage publié en langue anglaise en 1966). Paris: Armand Colin.

Cannon, W. B. (1929). Bodily changes in pain, hunger, fear and rage. New York: Appleton.

Festinger, L. (1957). A theory of cognitive dissonance. Stanford, CA/ Stanford University Press. Frijda, N. (1986). The emotions. Cambridge, U. K.: Cambridge University Press.

Horowitz, M. (1976). Stress response syndromes. New York: Aronson.

Janoff-Bulman, R. (1992). Shattered assumptions: Towards a new psychology of trauma. New York: Free Press.

Kulka, R. A., Schlenger, W. E., Fairbank, J. A., Hough, R. L., Jourdan, B. K., Marmar C. R., \& Weiss, D. S. (1990). Trauma and the Vietnam war generation. Report of findings from the National Vietnam Veterans Readjustment Study. New York, NY: Brunner/Mazel.

Lang, P. J. (1979). A bio-informational theory of emotional imagery. Psychophysiology, 16, $495-512$

Lang, P. J. (1993). From emotional imagery to the organization of emotion in memory. In N. Birbaumer \& A. Ohman (Eds.), The structure of emotion. Kirkland, WA: Hogrefe \& Huber. Langer, E. J. (1975). The illusion of control. Journal of Personality and Social Psychology, 32, 31 328.

94 Lerner, M. (1980). The belief in a just world. New York: Plenum Press.

Leventhal, H. (1984). A perceptual-motor theory of emotion. In L. Berkowitz (Ed.), Advances in Experimental Social Psychology, (Vol. 17, pp. I 17-182). New York: Academic Press.

Mesquita, B. (1993). Cultural variations in emotions. Unpublished doctoral dissertation, University of Amsterdam, The Netherlands.

Oatley, K. \& Jenkins, J. M. (1996). Understanding emotions. Oxford, U. K.: Blackwell.

Finkenauer, C., \& Rimé, B. (1998). Socially shared emotional experiences vs. emotional experiences kept secret: Differential characteristics and consequences. Journal of Social and Clinical Psychology, 17, 295-318.

Perloff, L. S. (1983). Perceptions of vulnerability to victimization. Journal of Social Issues, 39, 4|-6|. 
Philippot, P., \& Rimé, B. (1998). Social and cognitive processing in emotion: A heuristic for psychopathology. In W. F. Flack \& J. D. Laird (Eds.), Emotion in psychopathology. Theory and research, (pp. 114-129). Oxford, UK: Oxford University Press.

Rimé, B. \& Gérard, J. (200I). L'impact des événements traumtisants. Manuscrit non publié. Université de Louvain. Louvain-la-Neuve, Belgique.

Rimé, B., Herbette, G., \& Corsini, S. (2002). Social sharing of emotion. In S. R. Fussell (Ed.) The verbal communication of emotions: Interdisciplinary perspectives.(pp. | 85-208). Mawhaw, N. J.: Erlbaum.

Rimé, B., Finkenauer, C., Luminet, O., Zech, E., \& Philippot, P. (1998). Social Sharing of Emotion: New Evidence and New Questions. In W. Stroebe and M. Hewstone (Eds.), European Review of Social Psychology, (Vol. 9, pp. 145-189). Chichester: Wiley

Rimé, B., Hayward, M. S., \& Pennebaker, J. W. (1996). [Characteristics of recovered vs. unrecovered emotional experiences]. Unpublished raw data.

Rimé, B et Herbette, G. (sous presse). L'impact des émotions: Approche cognitive et sociale. In J.-M. Colletta \& A. Tcherkassof (Dir.), Perspectives actuelles sur les émotions. Cognition, langage et développement. Hayen: Mardaga.

Rimé, B., Mesquita, B., Philippot, P., \& Boca, S. (199/b). Beyond the emotional event: Six studies on the social sharing of emotion. Cognition and Emotion, 5, 435-465.

Rimé, B., Noël, P., \& Philippot, P. (199|a). Episode émotionnel, réminiscences mentales et réminiscences sociales. Les Cahiers Internationaux de Psychologie Sociale, 11, 93-104.

Rimé, B., Philippot, P., Boca, S., \& Mesquita, B. (1992). Long-lasting cognitive and social consequences of emotion: Social sharing and rumination. In W. Stroebe \& M. Hewstone (Eds.), European review of social psychology (Vol. 3, pp. 225-258). Chichester: Wiley.

Rimé. B. \& Scherer, K. R. (Eds.)(1990). Les émotions. Textes de base. Neuchâtel et Paris : Delachaux et Niestlé.

Rimé, B., Yogo, M., \& Pennebaker, J.W. (1996). [Social sharing of emotion across cultures]. Unpublished raw data.

Scherer, K. R. (1984). On the nature and function of emotion: A component process approach. In K. R. Scherer \& P. Ekman (Eds.), Approaches to emotion (pp. 293-317). Hillsdale, N]: Lawrence Erlbaum.

Singh-Manoux, A. \& Finkenauer, C. (200I). Cultural Variations in social sharing of emotions: An intercultural perspective on a universal phenomenon. Journal of Cross-Cultural Psychology, 32, 647-661.

Singh-Manoux, A. (1998). Les variations culturelles dans le partage social des émotions [Cultural variations in social sharing of emotions]. Unpublished doctoral dissertation, Université de Paris X-Nanterre, France.

Solomon, S., Greenberg, J., \& Pyszcsynski, T. (1991). A terror management theory of social behavior: The psychological functions of self-esteem and cultural world-views. In M. P. Zanna (Ed.), Advances in Experimental Social Psychology (Vol. 24, pp. 93- 159). New York: Academic Press.

Tait, R. \& Silver, R. C. (1989). Coming to term with major negative life events In J. S. Uleman \& J. A. Bargh (Eds.), Unintended thought (pp. 35I-382). New York: The Guilford Press.

Taylor, S. E. \& Brown, J. D. (1988). Illusion and well-being: a social psychological perspective on mental health. Psychological Bulletin, 103, 193-210.

Taylor, S. E., Kemeny, M. E., Reed, G. M., Bower, J. E., \& Gruenewald, T. L. (2000). Psychological resources, positive illusions, and health. American Psychologist, 55, 99- 109. 
Van der Kolk, B. A., Weisaeth, L. \& Van der Hart, O (1996). History of trauma in psychiatry. In B. A. Van der Kolk, A. C. McFarlane, \& L. Weisaeth (Eds.), Traumatic stress. The effects of overwhelming experience on mind, body, and society, (pp. 47-74). New York, NY: The Guilford Press.

Weinstein, N. D. (1980). Unrealistic optimism about future life events. Journal of Personality and Social Psychology, 5, 806-820.

Weinstein, N. D. (1996). Unrealistic optimism: Present and future. Journal of Social and Clinical Psychology, I5, I-8.

Yogo, M. \& Onoe, K. (1998). The social sharing of emotion among Japanese students. Poster session presented at ISRE '98, The Biannual conference of the International Society for Research on Emotion, held in Wuerzburg, Germany, August 4-8, 1998.

Zech, E. (1994). La gestion du deuil et la gestion des émotions. Mémoire de licence non publié. Faculté de Psychologie, Université de Louvain, Louvain-la-Neuve, Belgique. 
Pierre Gosselin

École de Psychologie, Université d'Ottawa

\section{LA RECONNAISSANCE DE L'EXPRESSION FACIALE DES ÉMOTIONS}

\section{INTRODUCTION}

Quels sont les développements récents qui ont nourri la réflexion dans le domaine de la reconnaissance de l'expression faciale des émotions? Trois questions nous sont apparues recevoir au cours des dernières années une attention particulière. Les deux premières font référence, en raison de leur ampleur, à des problématiques persistantes dans le domaine de l'émotion. Il s'agit de la question de la structure de l'information fournie par le comportement expressif et de celle de la portée universelle de cette information. Ces deux questions ont fait l'objet de débats théoriques récents et il nous apparait important de les évoquer. La troisième question concerne l'appréciation de l'authenticité de l'expression émotionnelle. Les travaux des dernières années ont permis de mieux documenter les mécanismes de régulation des comportements expressifs, comme le masquage, l'atténuation, l'amplification et la simulation des émotions. Dans le cadre de notre recension des travaux, nous examinerons la qualité des jugements dans l'appréciation de l'authenticité de l'expression ainsi que les facteurs qui la déterminent.

\section{LA STRUCTURE DE L'INFORMATION FOURNIE PAR L'ACTIVITÉ DU VISAGE}

L'une des grandes questions fondamentales de la psychologie de l'émotion concerne la structure des phénomènes qui sont désignés par ce terme (Frijda, 1986). Ce problème est celui qui a probablement le plus posé obstacle à l'étude psychologique des émotions. La raison principale semble 
en être la complexité des phénomènes couverts sous le terme émotion ainsi que la difficulté de les situer par rapport à ceux qui sont englobés dans le domaine, encore plus vaste, de l'affectivité.

Les discussions théoriques qui ont entouré cette question ont été stimulées récemment par une série d'articles publiés par Ortony et Turner (1990), Ekman (1992) et Izard (1992). Dans leur article, Ortony et Turner (1990) mettent en question l'existence des émotions fondamentales postulées par les théories psychoévolutionnistes. La critique des auteurs porte sur l'appui empirique de la primauté biologique et psychologique des émotions fondamentales, sur le caractère irréductible de celles-ci, sur le nombre d'émotions fondamentales et sur la possibilité d'expliquer la diversité de la vie affective à partir des émotions fondamentales. Cet article a été suivi d'une vive réaction des théoriciens psychoévolutionnistes (Ekman, 1992; Izard, 1992) qui ont fait valoir l'ampleur des données empiriques en faveur de l'existence des émotions fondamentales.

Selon la perspective psychoévolutionniste (Ekman, 1977, 1984, 1992; Izard, 1977, 1990; Plutchik, 1980; Tomkins, 1982), la vie émotionnelle est un tissu complexe composé d'émotions fondamentales et de structures affectives-cognitives. Les émotions fondamentales sont des catégories discrètes d'émotions et chacune de celles-ci se caractérise par un patron expressif, physiologique et phénoménologique qui lui est propre. De plus, chacune d'elles possède une fonction adaptative qui se présente sous la forme d'une tendance à l'action. L'expression et la reconnaissance des émotions fondamentales jouent un rôle important dans la régulation du comportement social de l'espèce. Elles favorisent la cohésion sociale et permettent une réduction des conflits interindividuels. Sept émotions fondamentales font l'objet d'un fort consensus parmi les théoriciens psychoévolutionnistes : la joie, la peur, la colère, la surprise, la tristesse, le dégoût et le mépris.

Dans leur plaidoyer en faveur des émotions fondamentales, Ekman (1992) et lzard (1992) font ressortir divers arguments qui attestent leur fondement biologique. Premièrement, il existe des changements physiologiques, notamment au niveau des systèmes nerveux périphérique et central, qui caractérisent les émotions fondamentales. Deuxièmement, les émotions fondamentales sont caractérisées par des expressions faciales qui sont universelles. Troisièmement, ces expressions faciales émergent au cours des premiers mois de la vie. Quatrièmement, ces émotions existent aussi chez 
d'autres primates. Cinquièmement, bien que la question du nombre exact d'émotions fondamentales demeure ouverte, le désaccord entre les théoriciens psychoévolutionnistes est moindre que ce qui est affirmé par Ortony et Turner (1990). II existe un fort consensus pour au moins six à sept émotions fondamentales.

Nous évoquons ici cette controverse sur l'existence des émotions fondamentales parce qu'elle est éminemment pertinente au domaine de la reconnaissance de l'expression émotionnelle. Elle nous conduit à nous questionner sur la structure de l'information fournie par les canaux expressifs.

Ekman (1992) et lzard (1992) présentent plusieurs arguments montrant que les canaux expressifs communiquent des émotions fondamentales. Ils présentent aussi plusieurs données montrant que certaines expressions faciales émotionnelles sont reconnues universellement. Cependant, ces données ne représentent que les premiers jalons d'une réponse à la question générale de la structure de l'information fournie par les canaux expressifs. Plusieurs questions plus spécifiques demeurent ouvertes. Ainsi, les caractéristiques des réponses expressives qui permettent aux décodeurs de les interpréter comme des émotions fondamentales sont encore peu documentées. Existe-t-il plusieurs expressions qui permettent de communiquer une même émotion fondamentale? Si oui, ces expressions communiquent-elles quelque chose de plus spécifique sur l'émotion? II est enfin une autre question qui demeure ouverte: celle de la valeur du concept de prototype dans l'étude de la reconnaissance des expressions émotionnelles. Dans les paragraphes qui suivent, nous exposerons et discuterons les résultats des études récentes qui ont tenté d'apporter une réponse à ces questions.

\section{La richesse du répertoire expressif}

L'idée selon laquelle une émotion fondamentale peut être communiquée par plusieurs expressions différentes a été suggérée par Ekman et Friesen (1978, 1986). Sur la base d'anályses rétrospectives de leurs études inter culturelles, ces auteurs ont proposé un répertoire facial des émotions fondamentales. Au total, près d'une centaine expressions sont comprises dans ce répertoire. La diversité des expressions varie selon l'émotion. On ne compte que quelques expressions pour le mépris et la joie, autour d'une 
dizaine pour la surprise et le dégoût, et au delà d'une vingtaine pour la peur, tristesse et la colère.

L'évaluation systématique des propositions d'Ekman et Friesen n'a été entreprise que très récemment. Les premières indications ont été rapportées par Wiggers (1982) qui a examiné la reconnaissance d'une dizaine d'expressions du répertoire ainsi que certaines de leurs variantes. Son étude fait mention d'un niveau élevé d'exactitude pour les expressions de joie, de colère, de surprise et de dégoût. Par contre, l'exactitude des jugements fut plutôt faible pour les expressions de peur et intermédiaire pour celles de la tristesse.

Plus récemment, Gosselin et Kirouac (1995) ont examiné la reconnaissance d'une trentaine d'expressions du répertoire d'Ekman et Friesen (1978). Les résultats montrèrent que les expressions de joie, de colère, de surprise et de tristesse furent très bien reconnues. Des résultats plus partagés furent cependant obtenus dans les cas de la peur et du dégoût. Seulement deux des six expressions de dégoût examinées furent reconnues avec une forte exactitude et aucune des six expressions de peur examinées ne fut fortement reconnue.

Les données actuelles portent donc à croire que plusieurs expressions peuvent communiquer une même émotion fondamentale. L'examen du répertoire facial est cependant loin d'être complété et d'autres recherches devront être conduites pour mieux apprécier la diversité des expressions qui peuvent communiquer les émotions fondamentales.

\section{La spécificité de l'information fournie par les expressions émotionnelles}

Le fait que plusieurs expressions peuvent communiquer une même émotion fondamentale nous conduit à la deuxième question que nous avons formulée plus haut: ces expressions communiquent-elles quelque chose de plus spécifique sur l'émotion? Les données disponibles sur cette question se limitent actuellement aux expressions du dégoût.

A l'intérieur d'une série de trois études, Rozin, Lowery et Ebert (1994) ont examiné l'interprétation que des décodeurs faisaient de neuf expressions faciales de dégoût. Plus spécifiquement, les décodeurs devaient choisir l'expression du visage qui correspondait le mieux à la réaction d'une personne dans diverses situations. Plus d'une cinquantaine de situations 
étaient décrites aux participants. Elles faisaient référence à des stimulations sensorielles désagréables ou irritantes (gustatives, olfactives et visuelles), à des pratiques sexuelles déviantes, à des pratiques hygiéniques malsaines, à des contaminations inter personnelles et à des offenses morales.

Les résultats de l'étude montrèrent que les expressions impliquant le plissement de la peau du nez était principalement associé à des stimulations olfactives désagréables, que l'ouverture de la bouche et l'extension de la langue étaient associées à des stimulations gustatives irritantes et que le relèvement de la lèvre supérieure était associé principalement à des situations sociales déplaisantes ou répugnantes (pratiques hygiéniques malsaines, relations sexuelles déviantes, offenses morales). L'étude de Rozin et al. (1994) appuie donc l'idée que les différentes expressions faciales d'une émotion fondamentale peuvent communiquer une information plus spécifique sur la nature de la situation qui provoque cette émotion.

\section{La notion de prototype facial}

La notion de prototype facial a été proposée pour désigner les expressions faciales qui sont les plus représentatives de chacune des émotions fondamentales et qui sont reconnues inter culturellement (Ekman \& Friesen, 1978; Ekman, Levenson \& Friesen, 1983). Bien que cette notion ait été proposée depuis plus d'une vingtaine d'années, les travaux de recherche n'ont pas encore permis de documenter de façon satisfaisante l'étendue et l'organisation de chacune des classes expressives. Les travaux de Wiggers (1982) et de Gosselin et Kirouac (1995) indiquent que plusieurs des prototypes proposés par Ekman et Friesen (1978) sont fortement reconnus par des observateurs. Cependant, cet appui empirique à la notion de prototype demeure assez modeste puisque rien ne garantit que d'autres configurations faciales que celles étudiées dans ces études ne seraient pas mieux reconnues. II conviendrait dans les années futures de mieux délimiter l'étendue des combinaisons d'unités d'action qui sont interprétées comme étant représentatives de chacune des émotions fondamentales et d'identifier parmi elles celles qui génèrent les niveaux de reconnaissance les plus élevés.

Par ailleurs, une meilleure connaissance des prototypes faciaux exigera que la structure même de ces signaux, et non seulement l'interprétation à 
laquelle ils peuvent donner lieu, soit examinée plus en détail. L'universalité de la reconnaissance de certaines configurations faciales a constituée jusqu'à présent la principale évidence de l'existence de prototypes. Le critère de reconnaissance ne pourrait cependant suffire à lui seul pour établir que les configurations en question sont effectivement émotionnelles. II se pourrait que les configurations faciales que des observateurs indépendants identifient comme étant les plus représentatives des émotions soient en fait des entités idéales, des constructions de l'appareil cognitif, et qu'elles ne sont jamais ou rarement observées dans la réalité. Cette interprétation a été proposée récemment par Carroll et Russell (1997) pour expliquer le fait que les prototypes faciaux sont rarement observés lorsque des émotions sont exprimées. Selon ces auteurs, les patrons d'activité faciale qui accompagnent les émotions seraient très variables, impliqueraient majoritairement des combinaisons d'un nombre réduit d'unités d'action et se caractériseraient par une structure componentielle (Scherer, 1984; Smith \& Scott, 1997). Référant aux divers travaux réalisés en sciences cognitives sur la formation des prototypes (Nosofsky, 1992; Posner \& Keele, 1968), Carroll et Russell émettent l'hypothèse que les prototypes faciaux correspondraient à des abstractions dans lesquelles se trouvent combinées un éventail plus large d'unités d'action que ce qui est observé dans la vie quotidienne. Une telle critique fait ressortir les limites des connaissances qui peuvent être acquises à partir des études de jugement et la nécessité d'étudier plus intensivement la structure physique des signaux émotionnels émis par l'être humain.

\section{L'UNIVERSALITÉ DE LA RECONNAISSANCE DE L'EXPRESSION FACIALE DES ÉMOTIONS}

L'universalité de l'information fournie par les canaux expressifs est la deuxième question qui a suscité de vives discussions au cours des dernières années. L'importance qu'on lui accorde est liée au fait qu'elle se rattache à la question plus large des déterminants biologiques de l'émotion.

Selon les théories psychoévolutionnistes (Ekman, 1977, 1984; Izard, 1977. 1990; Plutchik, 1980), l'expression spontanée des émotions résulte de l'activation d'un programme déterminé génétiquement. L'une des implications de ce déterminisme est qu'il existe un répertoire expressif commun à l'espèce humaine. Dans la mesure où ce répertoire existe, l'information qu'il 
véhicule doit avoir une portée transculturelle. Ceci dit, les versions récentes des théories psychoévolutionnistes admettent l'existence d'un déterminisme culturel, lequel affecterait la régulation du comportement émotionnel ainsi que la conceptualisation des émotions.

A ce déterminisme biologique, s'est opposée historiquement la thèse relativiste, principalement soutenue par des anthropologues, des sociologues et des psychologues sociaux (Birdwhistell, 1970; Harré, 1986; Lutz, 1982). La thèse relativiste met l'accent sur les variations culturelles qui caractérisent le comportement émotionnel. Les versions les plus extrêmes de cette thèse affirment que le comportement émotionnel est essentiellement un produit culturel, modelé par les valeurs, les croyances et l'organisation particulière d'un groupe social. Deux prédictions sont autorisées à partir de cette perspective. Premièrement, la reconnaissance des expressions émotionnelles doit se caractériser par l'existence de niveaux de compétences inégaux entre les cultures. En particulier, les individus d'un groupe social donné devraient mieux reconnaitre leurs propres expressions que celles des autres groupes. Deuxièmement, les niveaux de reconnaissance des expressions des autres groupes sociaux ne devraient être supérieurs au hasard que dans la mesure où l'apprentissage des normes culturelles de ces autres groupes a été possible. Les groupes sociaux isolés ne devraient pas pouvoir reconnaître les expressions émotionnelles des autres groupes.

La question de l'universalité de l'information fournie par les canaux expressifs a fait l'objet de vastes programmes de recherche inter culturelle à partir de la fin des années soixante (Ekman, Sorenson \& Friesen, 1969; Ekman et Friesen, 197I; Izard, 197I) et plusieurs recherches d'envergure ont eu lieu récemment (Ekman \& Friesen, 1986; Ekman et al., 1987; Haidt \& Kelner, 1999; Matsumoto \& Ekman, 1989, Russell, Lewicka \& Niit, 1989). En raison des difficultés méthodologiques rencontrées dans la description même du comportement expressif, la question de l'universalité des expressions émotionnelles a été principalement examinée à partir des études de reconnaissance.

Plusieurs analyses critiques ont été consacrées à cette littérature (Ekman, 1972, Ekman, 1982; Fridlund, 1994; Izard, 1971; Mesquita \& Frijda, 1992) et certaines d'entre elles ont pris un caractère polémique (Russell, 1994; Ekman, 1994; Izard, 1994). L'intérêt que présentent ces polémiques est multiple. Elles ont conduit d'une part à une reformulation plus précise de la thèse universaliste, en corrigeant notamment certaines erreurs d'interprétation 
dont elle a fait l'objet. Elles ont donné lieu d'autre part à une évaluation critique des données empiriques qui sont disponibles sur cette question, permettant, en autres, une mise en perspective des faits d'observation les plus saillants. Enfin, elles ont permis, dans une certaine mesure, de dégager de nouvelles orientations de recherche.

\section{La thèse universaliste}

À l'intérieur de leurs écrits, les défendeurs de la position universaliste (Ekman, 1994; Izard, 1994) sont d'abord conduits à identifier et à préciser les principales propositions théoriques de la perspective psychoévolutionniste. Selon cette perspective, l'expression spontanée des émotions est le résultat de l'activation d'un programme affectif faisant suite à la perception et à l'interprétation de certaines catégories d'événements. Ce programme affectif est déterminé génétiquement et permet une réaction rapide de l'organisme.

La première conséquence de l'existence d'un répertoire émotionnel commun à l'espèce humaine est le fait qu'il doit exister une certaine quantité d'expressions émotionnelles qui sont reconnues universellement. II ne s'agit pas de toutes les expressions émotionnelles, mais seulement de celles qui correspondent aux expressions spontanées faisant partie du répertoire. La mise à l'épreuve de cette proposition nécessite donc de la part des chercheurs des efforts particuliers dans la sélection des stimuli.

La deuxième précision concerne le critère de performance qui doit être utilisé pour évaluer la reconnaissance. Formellement, la reconnaissance implique un niveau de performance supérieur au hasard, ce qui, selon Ekman (1994), serait une condition suffisante. Plusieurs facteurs font qu'on ne peut

104 s'attendre d'emblée à une reconnaissance parfaite. D'une part, la conceptualisation des émotions diffère selon les cultures, ce qui fait qu'il n'est pas aisé de trouver des termes émotionnels équivalents d'une culture à l'autre. De plus, même à l'intérieur des collectivités qui partagent la même langue, un même mot peut avoir des connotations différentes. Enfin, les difficultés techniques qui se présentent dans la réalisation des études peuvent conduire à une sous-estimation des capacités des sujets. Ce problème se pose de façon plus particulière avec les populations isolées, étrangères aux études scientifiques. 
Enfin, les défenseurs de la thèse universaliste rappellent que la perspective psychoévolutionniste ne nie pas l'influence de la culture sur l'expression et la reconnaissance des émotions. La théorie neuroculturelle (Ekman, 1977, 1984) et la théorie des émotions différentielles (Izard, 1977, 1991) contiennent des propositions spécifiques touchant l'influence du milieu sur l'induction des émotions et sur la régulation des comportements expressifs. II en découle que l'existence de niveaux différents de reconnaissance entre les cultures n'est pas un argument qui contredit la thèse universaliste. II peut exister à la fois des expressions universelles et d'autres qui relèvent des acquisitions culturelles.

\section{Convergence des observations}

Suite aux analyses critiques de la littérature que l'on retrouve dans les derniers débats théoriques (Ekman, 1994; Izard, 1994, Russell, 1994), il est permis d'identifier plusieurs faits d'observation particulièrement robustes autour desquels se dégage un consensus entre les auteurs.

\section{Les membres de plusieurs pays occidentaux ou occidentalisés parviennent à bien reconnaître les expressions faciales des membres des autres pays.}

Le premier fait d'importance, et certainement le plus documenté, concerne la reconnaissance de l'expression faciale dans les pays occidentaux ou occidentalisés. Les études menées dans plus d'une trentaine de pays, en Europe, en Amérique et en Asie, indiquent que les membres d'un pays donné réussissent à reconnaître les expressions faciales des membres des autres pays (Ekman, 1972; Ekman \& Friesen, 1986; Ekman et al. 1987; Izard, 1971). Le niveau de performance des sujets n'est pas simplement supérieur au hasard, il est élevé pour la plupart des émotions fondamentales. Ce résultat est en accord avec la thèse universaliste, mais il n'en constitue qu'un appui partiel. En effet, comme la majorité des études ont porté sur les populations de pays industrialisés ayant des échanges culturels et commerciaux entre eux, les forts pourcentages de reconnaissance pourraient aussi traduire l'apprentissage des normes culturelles. 


\section{Les membres de populations isolées sur le plan culturel parviennent à reconnaître les expressions faciales des membres des autres collectivités.}

Les données les plus décisives proviennent des études faites auprès de populations isolées sur le plan culturel, c'est à dire de groupes culturels n'ayant pas eu l'occasion de faire l'apprentissage des normes culturelles des pays occidentalisés. De tels groupes existaient toujours dans le début des années 1970, notamment en Nouvelle-Guinée et à Bornéo. Les résultats de ces études montrent que plusieurs groupes culturels isolés (Fore, Dani, Sadong, Bahinemo) parviennent à reconnaître les expressions faciales des membres de cultures occidentalisées (Ekman, Sorenson \& Friesen, 1969; Ekman \& Friesen, 1971; Sorenson, 1975, 1976). Un tel résultat appuie la position universaliste et est difficilement conciliable avec la thèse relativiste.

\section{Le niveau de reconnaissance des expressions faciales varie en fonction des groupes culturels.}

Bien que les membres d'un groupe culturel parviennent à reconnaître les expressions faciales des autres groupes culturels, plusieurs études révèlent que le niveau de performance n'est pas uniforme d'une culture à l'autre pour certaines émotions (Izard, 1971; Ekman et al. 1987, Matsumoto, 1992; Matsumoto \& Ekman, 1989). C'est le cas, plus particulièrement, de la peur, de la colère et du dégoût. Ces différences pourraient refléter l'existence de variations culturelles dans l'encodage des expressions (Markham \& Wang, 1996). Elles pourraient aussi résulter de certains biais ethniques de jugement (Kilbride \& Warczower, 1983) ou de certaines règles culturelles de décodage (Biehl et al., 1997). II est également possible que ces différences soient attribuables à l'imperfection des mesures de la reconnaissance. Les variations culturelles qui existent dans la conceptualisation et le lexique des émotions (Matsumoto \& Assar, 1992) font qu'il n'est pas aisé d'assurer l'équivalence des tâches de jugement. Quant au niveau plus faible de reconnaissance atteint par les membres des groupes culturels isolés, il pourrait traduire, selon Ekman (1994), le 
manque de familiarité de ces groupes avec le rôle de participants dans des études scientifiques.

\section{Les études de jugement impliquant un choix forcé ont généré des niveaux de reconnaissance interculturelle plus élevés que celles faisant appel à une désignation verbale libre.}

Les études ayant rapporté les niveaux de reconnaissance interculturelle les plus élevés ont soumis les décodeurs à une tâche dans laquelle ils devaient choisir, parmi plusieurs termes émotionnels proposés, celui qui correspondait à l'expression faciale. Lorsque les sujets doivent choisir le terme émotionnel qui leur vient spontanément à l'esprit, le niveau d'accord est sensiblement plus faible. Cette observation est révélatrice, selon Russell (1994), des variations culturelles dans la conceptualisation des émotions et conséquemment dans le lexique propre à chaque langue. II importe toutefois de dire que l'on retrouve cette différence entre les deux types de tâche à l'intérieur d'un même groupe culturel. Elle traduirait donc plus fondamen-talement une variabilité dans la compréhension et l'utilisation du langage (Ekman, 1994; Izard, 1994). Une étude récente de Haidt et Keltner (1999) montre par ailleurs que les jugements produits avec la désignation verbale libre conduit à des résultats très convergents avec ceux obtenus avec la méthode de choix forcé, suggérant que les forts niveaux de reconnaissance obtenus avec ce dernier type de jugement ne sont pas le produit d'un artefact méthodologique.

Cette différence entre les deux tâches de jugement est intéressante parce qu'elle souligne à quel point le niveau d'analyse choisi a un impact sur la mise en évidence des universaux. Comme le soulignent Mesquita et Frijda (1992), la question de l'universalité peut être examinée à des niveaux plus ou moins abstraits. Les évidences les plus fortes de l'existence des universaux proviennent des études impliquant un niveau de traitement plus abstrait. C'est le cas lorsque la tâche de jugement implique un choix forcé. Les décodeurs doivent alors associer une expression faciale à une catégorie émotionnelle, c'est-à-dire à une entité abstraite à laquelle se trouvent subsumées des réalités plus concrètes. Lorsque la tâche de jugement implique une désignation verbale libre, les influences culturelles et interindividuelles se manifestent davantage, introduisant une variabilité qui peut masquer l'existence des universaux. 


\section{Direction future de la recherche}

Les études de jugement ont permis d'apporter plusieurs éléments de réponse à la question de l'universalité de la reconnaissance des expressions faciales des émotions. II est toutefois un aspect de la démonstration qui requiert une meilleure documentation. II s'agit du caractère véritablement émotionnel des expressions faciales qui ont généré de forts niveaux de reconnaissance sur le plan inter culturel. Ce point est probablement le plus crucial de ceux soulevés par les critiques de la position universaliste (Fridlund, 1994; Russell, 1994). II conviendrait de démontrer que ces expressions sont valides et qu'elles ont une valeur écologique. Si elles remplissent effectivement ces conditions, des études de composantes devraient révéler qu'elles ont une forte probabilité d'apparition lorsque des membres de cultures diverses expriment spontanément une émotion. Les indications de cette nature demeurent encore aujourd'hui peu nombreuses.

\section{LE JUGEMENT DE L'AUTHENTICITÉ DE L'EXPRESSION FACIALE DES ÉMOTIONS}

Les mécanismes de contrôle des comportements expressifs contribuent grandement à complexifier le processus de communication des émotions. Le fait que l'être humain puisse atténuer, amplifier, simuler et masquer son expression émotionnelle (Ekman, 1977, 1993) augmente considérablement la difficulté d'interprétation des messages émotionnels. Les conséquences du contrôle varient en fonction des stratégies particulières employées par l'encodeur. L'atténuation et l'amplification, par exemple, peuvent conduire le décodeur à sous estimer ou à surestimer l'intensité de l'émotion. Dans les cas extrêmes d'atténuation, il est même probable que l'émotion puisse ne pas être détectée. La simulation et le masquage, de leur côté, risquent d'engendrer une interprétation tout à fait erronée de la nature de l'émotion ressentie.

Les erreurs d'interprétation susceptibles de résulter des stratégies de contrôle ne sont pas nécessairement dommageables pour le décodeur. Selon Saarni et Lewis (1993), la dissimulation de l'émotion est fréquemment utilisée pour ménager les sentiments de l'entourage social et favoriserait de la sorte des échanges sociaux plus harmonieux. La capacité à détecter la tromperie devient cependant avantageuse lorsqu'elle permet au décodeur de se soustraire à des manipulations expressément destinées à lui nuire. 
Pour juger l'authenticité de l'expression émotionnelle, les décodeurs disposent d'une certaine quantité d'indices. Ainsi, les expressions faciales simulées sont plus souvent asymétriques que ne le sont les expressions authentiques (Ekman, Hager \& Friesen, 1981; Hager \& Ekman, 1985) et elles présentent une dynamique de contraction musculaire plus irrégulière (Hess \& Kleck, 1990; Weiss, Blum \& Gleberman, 1987). De leur côté, les expressions faciales produites pour masquer une émotion se distinguent des expressions authentiques par l'absence de certaines composantes faciales. Lorsqu'une personne ressentant la peur ou le dégoût simule la joie, la contraction de l'orbicularis oculi, produisant la levée des joues et tirant la peau autour des yeux vers l'intérieur des orbites oculaires, est moins fréquente que lorsque l'expression est authentique (Ekman, Friesen \& O'Sullivan, 1988).

Que nous révèlent les travaux portant sur l'appréciation de l'authenticité des expressions émotionnelles? Une première série de travaux ont porté sur la capacité des décodeurs à distinguer entre les expressions authentiques et les expressions de masquage. Par expression de masquage, nous désignons ici l'expression émotionnelle qui est simulée alors que l'encodeur ressent une émotion différente. Dans la plupart des travaux que nous rapportons, il s'agit de la simulation d'une émotion plaisante alors que l'émotion ressentie est déplaisante.

La majorité de ces travaux indiquent que les décodeurs sont capables, dans certaines conditions, de faire la distinction entre les deux classes d'expressions (DePaulo, 1988, Ekman \& Friesen, 1974; Ekman, O'Sullivan, Friesen \& Scherer, 1991; O'Sullivan, Ekman, Friesen \& Scherer, 1985; Zuckerman, Koestner \& Collela, 1985). En général, la sensibilité des décodeurs est très modeste, les taux de réussite ne dépassant guère $60 \%$ dans une tâche où le pourcentage attribuable au hasard est de $50 \%$. Le canal de communication affecte sensiblement la qualité des jugements. Le canal facial, notamment, conduit à des jugements moins exacts que ceux produits à partir des canaux gestuel (Ekman \& Friesen, 1974) et audio (Zuckerman et al., 1985).

Des niveaux de réussite à peu près comparables ont été obtenus dans la discrimination entre les expressions simulées et authentiques. Gosselin \& Kirouac (1994) rapportent des niveaux de performance variant entre 51\% et $62 \%$, selon les canaux de communication auxquels avaient accès les décodeurs. L'infériorité du canal facial par rapport au canal audio n'a pas été reproduite dans cette étude. II est probable que cette divergence des résultats reflète une différence entre le masquage et la simulation de 
l'émotion. La présence de l'émotion ressentie, dans le masquage, pourrait interférer avec la production de l'émotion simulée et cette interférence pourrait s'exercer plus fortement sur le canal vocal.

La présence d'un biais dans le jugement de l'authenticité contribue à la modeste performance que l'on retrouve. Tant dans les tâches impliquant la discriminations entre les expressions authentiques et de masquage (DePaulo, Stone \& Lassiter, 1985; Zuckerman, Koestner, Collela \& Alton, 1984) que celles impliquant la discrimination entre les expressions authentiques et simulées (Gosselin \& Kirouac, 1994; Gosselin, Kirouac \& Doré, 1995; Hess \& Kleck, 1994), les décodeurs sont enclins à croire en l'authenticité de l'expression. La signification de ce biais demeure obscure. L'une des interprétations possibles serait qu'il reflète une croyance selon laquelle les gens sont généralement honnêtes dans la vie quotidienne. Certains pensent même qu'une telle croyance représente un postulat indispensable à l'établissement et au fonctionnement des systèmes de communication.

II existe des différences interindividuelles importantes dans l'habileté à juger l'authenticité. Certaines personnes dont la profession implique des habiletés dans ce domaine réussissent nettement mieux que la population générale. C'est le cas des agents des services secrets américains dont la performance s'est avérée nettement supérieure au personnel des autres corps d'emploi (Ekman \& O'Sullivan, 1991).

Plusieurs facteurs semblent entrer en jeu dans la réussite au jugement de l'authenticité. D'abord, l'expérience avec les contextes de tromperie et l'attention prêtée au comportement non verbal semblent déterminantes. C'est ce que suggère la supériorité des agents des services secrets par rapport aux autres corps d'emploi. De plus, on note, chez cette population particulière, que les agents les plus étroitement impliqués dans les interrogatoires réussissent mieux que ceux dont le travail comporte principalement des tâches administratives (Ekman \& O'Sullivan, 1991).

La réussite à détecter la tromperie n'est pas reliée à la confiance que les décodeurs ont en leur propre jugement. Elle est plutôt reliée à la flexibilité du décodeur à fonder son jugement sur des indices comportementaux variés. Cette flexibilité est avantageuse parce que les indices révélateurs de la tromperie ne circulent pas toujours dans le même canal mais fluctuent d'un canal à l'autre en fonction du temps. Le problème rencontré par plusieurs décodeurs est qu'ils se concentrent sur des indices qui ne sont pas révélateurs de la tromperie, comme par exemple la direction 
du regard (Hess \& Kleck, 1994). Ils ont aussi tendance à confondre les signes de nervosité avec les indices de tromperie. Comme des encodeurs peuvent à la fois exprimer honnêtement leurs émotions et manifester de la nervosité, cette confusion réduit l'exactitude de leur jugement.

Certaines conditions permettent aux décodeurs d'accroître la qualité de leur jugement. O'Sullivan, Ekman et Friesen (1988) et Frank et al. (1993) rapportent que la performance des décodeurs est meilleure lorsqu'on leur présente successivement deux expressions, l'une honnête et l'autre trompeuse, et qu'ils doivent ensuite identifier celle qui est trompeuse. On peut penser que cette condition de jugement facilite le rappel des informations pertinentes en mémoire et conduit à une analyse comparative plus complète. Les résultats rapportés par O'Sullivan et al. indiquent aussi que l'ordre de présentation des expressions affecte la performance des décodeurs. Ces derniers réussissent mieux lorsqu'ils voient d'abord l'expression authentique et ensuite l'expression trompeuse. Selon les auteurs, ce résultat serait révélateur du processus de décision habituellement utilisé par les décodeurs, processus qui consiste à prendre l'expression authentique comme référence et à évaluer les expressions trompeuses en regard de cette référence.

Le dernier aspect sur lequel nous nous pencherons concerne la possibilité d'accroître l'exactitude des jugements dans l'appréciation de l'authenticité. Zuckerman, Koestner et Collela (1985) ont examiné l'apprentissage par rétroaction sur la détection de la tromperie et rapportent un accroissement de la performance dans les trois conditions de canaux comprises dans leur étude (facial, audio et facial+audio). De plus, l'apprentissage se généralise à d'autres encodeurs que ceux visés par la rétroaction. II faut souligner toutefois que les gains sont modestes, particulièrement pour le canal facial, et que la performance des sujets, tout en excédant le niveau attribuable au hasard, demeure très imparfaite.

En conclusion, les études de jugement portant sur l'authenticité de l'expression émotionnelle sont révélatrices de deux faits principaux. En premier lieu, elles indiquent que le jugement de l'authenticité de l'expression émotionnelle en est un qui est imparfait. L'apprentissage dans ce domaine est possible, mais il implique un entraînement extensif, une sensibilité et une attention particulières aux indices verbaux et non verbaux, et de la flexibilité dans l'utilisation de ces indices. En second lieu, il apparait que le contrôle de l'expression émotionnelle est une entreprise assez réussie, permettant 
à l'encodeur, dans près de $40 \%$ à $50 \%$ des cas, de cacher à ses congénères son véritable état émotionnel.

\section{RÉFÉRENCES}

Bielh, M., Matsumoto, D., Ekman, P., Hearn, V., Heider, K., Kudoh, T., \& Ton, V. (1997). Matsumoto and Ekman's japanese and caucasian expressions of emotion (JACFEE): Reliability data and cross-national differences. Journal of Nonverbal Behavior, 21, 3-21.

Birdwhistell, R. L. (1970). Kinesics and context. Philadelphia: University of Pennsylvania Press. Carroll, J. M., \& Russell, J. A. (1997). Facial expressions in Hollywood's portrayal of emotion. Journal of Personality and Social Psychology, 72, 164-176.

DePaulo, B. M. (1988). Nonverbal aspects of deception. Journal of Nonverbal Behavior, 12, I53-161.

DePaulo, B. M., Stone, J. I., \& Lassiter, G. D. (1985). Deceiving and detecting deceit. In B. R. Schlenger (Ed.), (pp. 323-370). The self and social life. New York: McGraw-Hill.

Ekman, P. (1972). Universals and cultural differences in facial expressions of emotion. In J. Cole (Ed.), Nebraska Symposium on Motivation (pp. 207-283). Lincoln: University of Nebraska Press.

Ekman, P. (1977). Biological and cultural contribution to body and facial movement. In J. Blacking (Ed.). The anthropology of the body. San Diego: Academic Press, p. 34-84.

Ekman, P. (1982). Emotion in the human face. New York: Pergamon Press.

Ekman, P. (1984). Expression and the nature of emotion. In K. Scherer \& P. Ekman (Eds.). Approaches to emotion. Hillsdale: Erlbaum, p. 319-344.

Ekman, P. (1992). Are there basic emotions?. Psychological Review, 99, 550-553.

Ekman, P. (1993). Facial expression and emotion. American Psychologist, 48, 384-392.

Ekman, P. (1994). Strong evidence for universals in facial expressions: A reply to Russell mistaken critique. Psychological Bulletin, II 5, 268-287.

Ekman, P., \& Friesen, W.V. (197I). Constants across cultures in the face and emotion. Journal of Personality and Social Psychology, 17, 124-129.

Ekman, P., \& Friesen, W. V. (1974). Detecting deception from the body or face. Journal of Personality and Social Psychology, 29, 288-298.

Ekman, P., \& Friesen, W. V. (1978). Facial Action Coding System: Part Two. Palo Alto: Consulting Psychologists Press.

Ekman, P., \& Friesen, W.V. (1986). A new pan-cultural expression of emotion. Motivation and Emotion, 10, 159-168.

112 Ekman, P., Friesen, W.V., \& O'Sullivan, M. (1988). Smiles when lying. Journal of Personality and Social Psychology, 54, 4|4-420.

Ekman, P., Friesen, W.V., O'Sullivan, M., Chan, A., Diacoyanni-Tarlatzis, I., Heider, K., Krause, R., Lecompte, W.A., Pitcairn, T., Ricci-Bitti, P. E., Scherer, K., Tomita, M., \& Tzavaras, A. (1987). Universals and cultural differences in the judgments of facial expressions of emotion. Journal of Personality and Social Psychology, 53, 7|2-7|7.

Ekman, P., Hager, J. C., \& Friesen, W. V. (1981). The symmetry of emotional and deliberate facial actions. Psychophysiology, 18, 101-106.

Ekman, P., Levenson, R. W., \& Friesen, W. V. (1983). Autonomic nervous system activity distinguishes among emotions. Science, 221, 1208-1210.

Ekman, P. \& O'Sullivan, M. (199I). Who can catch a liar? American Psychologist, 46, 913-920. 
Ekman, P., O'Sullivan, M. B., Friesen, W. V., \& Scherer, K. R. (199I). Face, voice and body in detecting deceit. Journal of Nonverbal Behavior, I5, 125-135.

Ekman, P., Sorenson, E. R., \& Friesen, W. V. (1969). Pan-cultural elements in facial displays of emotions. Science, 164, 86-88.

Fehr, B., \& Russell, J. A. (1984). Concept of emotion viewed from a prototype perspective. Journal of Experimental Psychology: General, 113, 464-486.

Frank, M. G., Ekman, P., \& Friesen, W.V. (1993). Behavioral markers and recognizability of the smile of enjoyment. Journal of Personality and Social Psychology, 64, 83-93.

Fridlund, A. J. (1994). Human facial expression: An evolutionary view. San Diego: Academic Press.

Frijda, N. H. (1986). The emotions. New York: Cambridge University Press.

Gosselin, P., \& Kirouac, G. (1994). Effet du canal de communication sur le jugement d'émotions ressenties et simulées. Revue Canadienne des Sciences du Comportement, 26, 163-181.

Gosselin, P., \& Kirouac, G. (1995). Le décodage de prototypes émotionnels faciaux. Revue Canadienne de Psychologie Expérimentale, 49, 313-329.

Gosselin, P., Kirouac, G., \& Doré, F.Y. (1995). Components and recognition of facial expressions in the communication of emotion by actors. Journal of Personality and Social Psychology, 68, 83-96.

Hager, J. C., \& Ekman, P. (1985). The asymmetry of facial actions is consistent with models of hemispheric specialization. Psychophysiology, 22, 307-318.

Haidt, J., \& Keltner, D. (1999). Culture and facial expression: Open-ended methods find more expressions and a gradient of recognition. Cognition and Emotion, 13, 225-266.

Harré, R. M. (1986). The social construction of emotion. Oxford: Basil Blackwell.

Hess, U., \& Kleck, R. E. (1990). Differentiating emotion elicited and deliberate emotional facial expressions. European Journal of Social Psychology, 20, 369-385.

Hess, U., \& Kleck, R. E. (1994). The cues decoders use in attempting to differentiate emotion-elicited and posed facial expressions. European Journal of Social Psychology, 24, 367-38I.

Izard, C. E. (197I). The face of emotion. New York: Appleton-Century-Crofts.

Izard, C. E. (1977). Human emotions. New York: Plenum Press.

Izard, C. E. (1990). Facial expressions and the regulation of emotions. Journal of Personality and Social Psychology, 58, 487-498.

Izard, C. E. (199|). The psychology of emotion. New York: Plenum Press.

Izard, C. E. (1992). Basic emotions, relations among emotions, and emotion-cognition relations. Psychological Review, 99, 561-565.

Izard, C. E. (1994). Innate and universal facial expressions: Evidence from developmental and cross-cultural research. Psychological Bulletin, 1 15, 288-299.

Kilbride, J. E., \& Yarczower, M. (1983). Ethnic bias in the recognition of facial expressions Journal of Nonverbal Behavior, 8, 27-4I.

Lutz, C. (1982). The domain of emotion words on Ifaluk. American Ethnologist, 9, I I3- I 28.

Markham, R., \& Wang, L. (1996). Recognition of emotion by Chinese and Australian children. Journal of Cross-Cultural Psychology, 27, 616-643.

Matsumoto, D. (1992). More evidence for the universality of a contempt expression. Motivation and Emotion, 16, 363-368.

Matsumoto, D., \& Assar, M. (1992). The effects of language on judgments of universal facial expressions of emotion. Journal of Nonverbal Behavior, 16, 85-99.

Matsumoto, D., \& Ekman, P. (1989). American-Japanese cultural differences in intensity ratings of facial expressions of emotion. Motivation and Emotion, 13, 143-157. 
Mesquita, B., \& Frijda, N. H. (1992). Cultural variations in emotions: A review. Psychological Bulletin, 1 12, 179-204.

Nosofski, R. (1992). Similarity scaling and cognitive process models. Annual Review of Psychology, 43, 25-53.

Ortony, A., \& Turner, T. J. ( 1990). What's basic about basic emotions? Psychological Review, 97, $3|5-33|$.

O'Sullivan, M., Ekman, P., \& Friesen, W.V. ( 1988$)$. The effect of comparisons on detecting deceit. Journal of Nonverbal Behavior, 12, 203-214.

O'Sullivan, M., Ekman, P., Friesen, W. V., \& Scherer, K. R. (1985). What you say and how you say it:The contribution of speech content and voice quality to judgments of others. Journal of Personality and Social Psychology, 48, 54-62.

Plutchik, R. (1980). Emotion: A psychoevolutionary synthesis. New York: Harper and Row.

Posner, M. I., \& Keele, S.W. (1968). On the genesis of abstract ideas. Journal of Experimental Psychology, 77, 353-363.

Rozin, P., Lowery, L., \& Ebert, R. (1994). Varieties of disgust faces and the structure of disgust. Journal of Personality and Social Psychology, 66, 870-88I.

Russell, J. (1991). In defense of a prototype approach to emotion concepts. Journal of Personality and Social Psychology, 60, 37-47.

Russell, J. A. (1994). Is there universal recognition of emotion from facial expression? A review of the cross-cultural studies. Psychological Bulletin, 115, 102-141.

Russell, J. A., Lewicka, M., \& Niit, T. (1989). A cross-cultural study of a circumflex model of affect. Journal of Personality and Social Psychology, 57, 848-856.

Saarni, C., \& Lewis, M. (1993). Deceit and illusion in human affairs. In M. Lewis et C. Saarni (Eds.), Lying and deception in everyday life (pp. 1-29). New York: Guilford Press.

Scherer, K. R. (1984). On the nature and function of emotion: A component process approach. In K. R. Scherer \& P. Ekman (Eds.), Approaches to emotion (pp.293-3 I8). Hillsdale: Lawrence Erlbaum Associates.

Smith, C. A., \& Scott, H. S. (1997). A componential approach to the meaning of facial expressions. In J. A. Russell \& J. M. Fernandez-Dols (Eds.), The psychology of facial expression (pp. 229-254). Cambridge (UK): Cambridge University Press.

Sorenson, E. R. (1975). Culture and the expression of emotion. In T. R. Williams (Ed.), Psychological anthropology (pp. 361-372). Chicago: Aldine.

Sorenson, E. R. (1976). The edge of the forest: Land, chilhood and change in a New Guina protoagricultural society. Washington, DC: Smithonian Institution Press.

Tomkins, S. S. (1982). Affect theory. In P. Ekman (Ed.), Emotion in the human face (pp.). New York: Cambridge University Press.

Weiss, F., Blum, G. S., \& Gleberman, L. (1987). Anatomically based measurement of facial expressions in simulated versus hypnotically induced affect. Motivation and Emotion, II, 67-81.

Wiggers, M. (1982). Judgments of facial expressions of emotion predicted from facial behavior. Journal of Nonverbal Behavior, 7, $101-116$.

Zuckerman, M., Koestner, R., \& Colella, M. J. (1985). Learning to detect deception from three communication channels. Journal of Nonverbal Behavior, 9, 188-194.

Zuckerman, M., Koestner, R., Colella, M. J., \& Alton, A. O. (1984). Anchoring in the detection of deception and leakage. Journal of Personality and Social Psychology, 47, 301-31 I. 


\section{Ursula Hess}

Département de Psychologie

Université du Québec à Montréal

\section{ÉMOTION RESSENTIE ET SIMULÉE}

\section{Introduction}

Cet article traitera des différences entre les émotions spontanées et les émotions simulées. Une telle distinction suscite la question de savoir si elle est utile sur le plan épistémologique et si oui, comment peut-on définir et distinguer les deux concepts ainsi proposés? Dans un premier temps, ces questions seront examinées et quelques réponses seront suggérées. Dans un deuxième temps, je présenterai quelques résultats empiriques qui portent sur cette différenciation et je tenterai d'intégrer ces informations dans un cadre qui considère les émotions simulées selon une perspective interactionnelle.

\section{Qu'est-ce qu'une émotion simulée?}

Afin de déterminer les caractéristiques des émotions simulées, il faudrait d'abord s'entendre sur la définition du terme émotion. Un grand nombre de définitions ont été proposées, dont plusieurs partagent des points communs. Récemment, les théories cognitives qui considèrent les émotions comme des produits d'un processus d'évaluation cognitive ont eu préséance dans la littérature (e.g. Scherer, 1986; Frijda, 1986; Lazarus, 1991). Toutefois, des positions fonctionnalistes ont aussi été ardemment défendues (e.g. Tomkins, 1962, 1963, 1991, 1993; Plutchik, 1962, 1980; Ekman, 1984; Izard, 1971, 1991). Ces deux approches s'entendent pour dire que les émotions sont caractérisées par des sensations subjectives, des réactions physiologiques, ainsi que des comportements verbaux et nonverbaux qui peuvent être ou non synchronisés. La majorité de ces définitions s'entendent sur le fait que les sensations subjectives sont une composante essentielle de l'émotion 
(Izard, 1990). De plus, il a été noté que les émotions se manifestent en réponse à certains types de stimuli (e.g. la tristesse comme réponse à une perte irrévocable, Lazarus, 1991). On pourrait donc dire que les émotions simulées sont des émotions qui apparaissent en l'absence de ces stimuli.

\section{Feeling rules}

Dans ce contexte, nous pouvons penser aux «feeling rules» proposées par Alice Hochschild (e.g., 1979; 1983). Cette dernière remarque que la société moderne exige de plus en plus que ses membres ressentent des émotions particulières dans certains contextes. Par exemple, elle note que plusieurs s'attendent à ce que dans le milieu de la santé les intervenants manifestent de la détresse et de la sympathie face au sort de leurs patients, tandis que d'autres exigent que les réceptionnistes et les agents de bord ressentent des émotions positives lors de leurs interactions avec leurs clients. Les «feeling rules» nécessitent que l'individu vive, et non seulement simule, l'émotion en question. Ainsi, la fatigue du passager ou la détresse du patient devraient être en soi des stimuli appropriés pour induire l'émotion culturellement appropriée et en conséquence leur affichage.

En effet, les demandes culturelles exigeant une certaine réaction émotionnelle face à une certaine classe des stimuli, cela devrait conduire automatiquement à l'expression d'une émotion accompagnée par l'état affectif correspondant. Étant donné que le sentiment est l'élément central pour la définition d'une émotion, l'émotion simulée ne pourrait donc être qu'une expression émotionnelle simulée.

\section{Règles d'affichage}

Les «display rules» ou «règles d'affichage des émotions», requièrent la simulation et la dissimulation des émotions. Ces règles d'affichage sont liées aux interactions sociales et guident l'affichage de certaines émotions dans des contextes précis. Ainsi, nous devrions ressentir de la joie lorsqu'on nous présente un cadeau, mais non à des funérailles. Cette idée que des normes sociales guident l'expression des émotions a été proposée parWundt (1903) et plus récemment, par Ekman et Friesen (1971). Ces auteurs avancent que 
ces règles sont spécifiques à chaque culture, mais que l'état affectif sous-jacent ne l'est pas. Ils présument alors, par example, que la majorité des individus ressentent de la colère face à un affront. Toutefois, dans certaines cultures ou sous-cultures, l'expression publique de cette colère pourrait être inhibée. D'autres chercheurs affirment plutôt que l'émotion comme telle est sociale et qu'elle est médiée par des connaissances culturelles. Chaque culture aurait ainsi ses propres déclencheurs d'émotions et pourrait définir les émotions de manière distincte (voir Markus \& Kitayama, 1994; Wierzbicka, 1994).

Des preuves existent pour valider ces deux perspectives. Des événements qui suscitent une émotion et les thèmes relationnels centraux proposés par Lazarus semblent être différents dans diverses cultures (e.g. Wierzbicka, 1995). De plus, les membres d'une même culture n'endossent pas tous l'expression de certaines émotions dans des contextes particuliers : par exemple, les femmes ont tendance à exprimer plus de peur que les hommes. En outre, les hommes et les femmes expriment une même émotion de manière différente. Par exemple, les femmes ont plus tendance à pleurer quand elles sont en colère que les hommes (Fischer, 1993). Ces derniers propos mettent en relief la dissociation possible entre l'état interne et l'affichage externe. Les contraintes culturelles qui demandent l'affichage d'une certaine émotion ou bien un certain type d'affichage d'émotions suscitent des expressions émotionnelles simulées. Dans une perspective interactionnelle, ces expressions simulées jouent le rôle d'émotions simulées.

L'expression des émotions simulées s'apprend tôt dans le processus de la socialisation de l'enfant. Les enfants peuvent utiliser les règles d'affichage dès leur jeune âge. Par exemple, Cole (1986) a démontré que des filles de 3 à 4 ans tentent de masquer leur déception lorsqu'elles reçoivent un cadeau indésirable. De même, Reissland et Harris (1991) ont trouvé que, même à 58 mois, des enfants essayent de cacher leur fierté dans certaines situations. Ces règles d'affichage peuvent être verbalisées par des enfants vers l'âge de six ans (e.g. Saarni, 1979).

\section{L'affichage d'émotions: symptômes ou signaux?}

La notion d'expressions émotionnelles simulées suppose qu'il existe une classe d'expressions qui représentent fiablement l'état émotif de l'émetteur. Ainsi, dans ce domaine de recherche, il y a une prémisse de base qui affirme qu'en 
temps normal, les expressions faciales émotionnelles agissent comme une représentation fidèle de l'état émotionnel de l'émetteur. Cette perspective date de l'Antiquité et Darwin (1872) déjà a décrit et souligné les similarités entre les diverses expressions émotionnelles chez l'humain et les animaux.

Récemment, toutefois, l'idée que les expressions émotionnelles faciales puissent être mieux comprises comme étant des communications d'intentions, modulées par la présence des autres et indépendantes de l'état émotionnel concomitant, a attiré l'attention de plusieurs chercheurs (Chovil, I99I; Fridlund, 1991; Fridlund, Kenworthy, \& Jaffey, 1992). Cette proposition est basée sur l'écologie comportementale qui affirme que - puisque les expressions faciales émotionnelles servent évidemment une fonction communicative et qu'on peut démontrer qu'elles peuvent être modifiées en présence d'un public l'explication voulant que les expressions faciales émotionnelles soient des symptômes d'un état affectif sous-jacent devient désuète.

Selon Fridlund et al. (1992), «l'effet d'auditoire» apporte un soutien empirique important à cette proposition, puisque ce phénomène souligne le caractère social de l'affichage émotionnel. Dans une série d'études, il a démontré, avec l'aide de ses collègues (Fridlund, 1991; Fridlund et al., 1992), que l'affichage d'expressions faciales négatives et positives subit une augmentation en présence d'un public réel ou imaginé. Fridlund, à partir de ses données, en vient à la conclusion que l'intensité des expressions faciales émotionnelles peut être prédite avec plus d'efficacité par le contexte social que par l'état affectif. II ajoute que ces résultats démontrent la nécessité de rejeter la notion «romantique» voulant que les expressions faciales soient une représentation fidèle et adéquate de l'état affectif sous-jacent.

La majorité des arguments principaux de Fridlund ne sont pas nouveaux. Le milieu de la recherche a reconnu, et ce depuis longtemps, que les expressions faciales peuvent être utilisées comme des symboles (Ekman, 118 1979), comme des interjections (Motley, 1993) et comme des signaux qui indiquent que l'individu comprend l'état émotionnel de son interlocuteur (Bavelas et al., 1986). En effet, il est évident que les expressions faciales, comme la plupart des messages, peuvent servir différentes fonctions en même temps. Cette proposition a généralement été véhiculée par ceux qui prétendent que les expressions faciales sont des symptômes d'un état affectif sous-jacent (e.g., Buck, 199I, Buck et al., 1992; Cacioppo, Bush, \& Tassinary, 1992; Hess, Kappas, \& Banse, 1995; Hess, Kappas, Kleck, McHugo, \& Lanzetta, 1989; Hess \& Kleck, 1990). 
Ainsi, le rôle des expressions faciales comme signaux d'interaction n'exclut pas leur rôle comme symptômes d'états émotionnels. De plus, il existe des données neurologiques qui montrent des différences de mécanismes entre les expressions faciales provoquées par un état émotionnel et celles qui sont produites volontairement. Plus particulièrement, les expressions faciales qui correspondent à un état affectif sous-ajacent semblent être innervées par des voies neuronales différentes que les expressions faciales simulées (voir Rinn, 1984, 1991). Par ailleurs, des résultats découlant d'études menées auprès des patients ayant une section du corps calleux démontrent que bien que les deux hémisphères puissent générer des expressions faciales spontanées, seule l'hémisphère gauche peut générer des expressions volontaires (Gazzaniga \& Smylie, 1990).

\section{L'effet d'auditoire réexaminé}

Nous avons illustré la fonction des expressions faciales émotionnelles comme représentations d'un état affectif et comme signal social (Hess, Banse, \& Kappas, 1995). Pour cela, nous avons reproduit l'expérience de Fridlund (|99|) en variant et l'aspect social du contexte et l'intensité du stimulus émotionnel, ainsi que la relation entre l'individu appelé à exprimer une émotion et son public. Les résultats indiquent que l'intensité de l'expression ne peut pas être prédite de manière satisfaisante par un seul de ces facteurs, mais plutôt par un mélange complexe des trois. Spécifiquement, nous avons trouvé que, lorsque les sujets regardaient des films qui variaient dans leur contenu humoristique soit en présence d'un copain, soit lorsque le copain fait la même tâche, mais dans une autre salle, ou lorsque le copain fait une autre tâche dans une salle différente, l'activité du Orbicularis Occuli (qui indique la présence d'un sourire) variait en fonction et du contenu humoristique et en fonction du degré de présence du «public». Ainsi, les sujets souriaient plus en regardant le film plus drôle que lorsqu'ils regardaient le film plus sérieux. De plus, les sujets souriaient plus souvent lorsqu'ils étaient en compagnie de leur ami que lorsque ce dernier travaillait à une tâche différente dans une autre salle. II n'y avait pas de différences notables lorsque le compagnon du sujet était un étranger. Alors, dans ce contexte, les expressions faciales ont servi de signaux pour communiquer le degré d'amusement de l'émetteur à son ami, en plus de représenter son état émotionnel. 


\section{Définition des expressions émotionnelles simulées}

Toutefois, les réserves émises par Fridlund (199|) concernant les expressions émotionnelles spontanées et simulées doivent être prises en considération. J'ai déjà proposé l'idée (Hess \& Kleck, 1990) qu'une simple séparation dichotomique des expressions en deux catégories, soit les expressions «senties» et les expressions «fausses», classification courante dans la littérature portant sur ce domaine (e.g., Ekman \& Friesen, 1982), ne rend pas justice à la complexité du phénomène. Au contraire, les expressions faciales émotionnelles spontanées et les expressions faciales émotionnelles voluntaires peuvent être considérées comme des extrêmes d'un même continuum.

Dans ce continuum, les expressions spontanées correspondent à l'état émotionnel de la personne et ne subissent pas ou peu de contrôle. Par contre, les expressions simulées sont des expressions qui ont une composante volontaire. Ces expressions peuvent être soit des expressions correspondant à l'état émotionnel de la personne, mais qui sont augmentées ou diminuées en fonction des contraintes de la situation, ou soit des expressions qui ne correspondent pas du tout à l'état émotionnel de l'individu.

Par conséquent, les expressions faciales émotionnelles peuvent être situées dans un espace bi-dimensionnel où une des dimensions représente l'état émotionnel de l'émetteur et l'autre dimension, l'émotion exprimée.

Les expressions émotionnelles spontanées reflètent la valence et l' intensité de l'état affectif de l'émetteur. Les expressions émotionnelles simulées soit peuvent varier en intensité, mais non en valence selon l'état affectif de l'émetteur (i.e. des expressions inhibées ou exagérées), soit, peuvent ne pas correspondre à l'état affectif de l'émetteur. Dans ce dernier cas, on parle de dissimulation.

L'affichage d'émotions simulées et la tromperie volontaire ne sont pas des termes équivalents. La tromperie volontaire, par définition, suppose que l'émetteur veuille tromper le récepteur, mais la simulation peut se produire dans d'autres circonstances. Principalement, en suivant les règlements d'affichage, les gens peuvent vouloir augmenter leur estime de soi. Ainsi, en masquant une émotion (e.g., la colère), un individu peut vouloir démontrer un comportement approprié, sans nécessairement vouloir consciemment tricher l'autre. Cette inhibition peut même se produire lorsque l'individu est seul et ne se fait pas observer. Dans cette circonstance, c'est cette conscience de vouloir bien agir qui motive le comportement. 
Par ailleurs, une école de pensée récente a ravivé la question entourant les émotions inconscientes, c'est-à-dire, les émotions qui pourraient être réprimées de la conscience rationnelle tout en laissant des traces dans le domaine nonverbal. Dans ces cas-là, la distinction entre la dissimulation volontaire - bien que non réussie - et la répression, est difficile à faire (Ekman, 1992; Lazarus, 1995). De plus, dans la mesure où l'état affectif subjectif est jugé comme étant la composante principale de l'émotion (voir Izard, 1990), le raisonnement devient même tautologique.

Aussi, certaines expressions volontaires peuvent se produire spontanément, comme c'est le cas des réactions surapprises qui s'apparentent à un réflexe. Un exemple de ce type de réaction serait le sourire émis pour saluer une connaissance. Ce sourire ne correspond pas nécessairement à un état réel de joie, mais fait plutôt partie d'un protocole social. Par conséquent, il est rarement interprété comme étant un signe indiquant l'état affectif de l'émetteur.

En résumé, pour classifier les expressions émotionnelles simulées comme étant une tromperie, il faut prendre en considération la motivation et l'état subjectif de l'émetteur et les attributions du receveur. C'est donc dire que, dans des situations où l'émetteur et le receveur partagent les mêmes règles culturelles s'entendent sur l'exactitude de l'expression compte tenu de la situation et reconnaissent l'expression comme un symbole plutôt qu'une représentation d'un état émotif (comme c'est le cas pour le sourire de salutation), alors il n'y a pas tromperie.

Il faut noter que le rôle actif de l'observateur dans ce processus a été largement ignoré par les chercheurs malgré le fait que la notion d'émotions simulées suppose implicitement la présence d'un observateur.

\section{L'encodage et le décodage d'expressions émotionnelles intentionnelles}

L'encodage d'expressions faciales émotionnelles subit l'influence d'une série de facteurs, incluant les contraintes sociales telles les règles d'affichage. Certains résultats émanant de notre laboratoire semblent indiquer que le décodage des expressions faciales émotionnelles peut subir des influences similaires.

La majorité des études qui traitent des expressions faciales intentionnelles se concentrent sur l'encodage et le décodage de messages trompeurs plutôt que sur les expressions émotionnelles volontaires comme telles. En ce qui a trait à l'encodage des expressions émotionnelles intentionnelles, la plupart 
des recherches ont porté sur les sourires. Duchenne avait déjà noté, en 1863, que les sourires spontanés sont caractérisés par une activité du muscle Orbicularis Oculi qui produit des rides autour des yeux. Ces résultats ont été confirmés par Ekman et ses collègues (Ekman \& Friesen, 1982; Ekman, Friesen, \& O'Sullivan, 1988; Ekman, Davidson, \& Friesen, 1990). Hess et al. (1989) ont aussi trouvé des différences entre les sourires spontanés et intentionnels en utilisant l'électromyographie faciale. De plus, il a été remarqué que les expressions volontaires sont plus asymétriques que les expressions spontanées (Hagar \& Ekman, 1985). Enfin, les sourires spontanés et volontaires ont des parcours temporels différents (Bugenthal, 1986; Ekman \& Friesen, 1982; Weiss, Blum, \& Gleberman, 1987; Hess \& Kleck, 1990). En somme, il existe de résultats démontrant que les sourires spontanés et délibérés diffèrent. Par conséquent, on pourrait s'attendre à ce que les décodeurs puissent distinguer les expressions de joie qui sont simulées de celles qui sont spontanées.

Toutefois, il n'en est pas ainsi. En général, les chercheurs ont remarqué que les gens ne sont pas très habiles lorsque vient le temps d'identifier les expressions simulées. Dans une importante étude, Ekman et O'Sullivan (1991) a tenté de vérifier cette capacité chez des gens de différentes professions pour qui de bonnes habiletés de décodage peuvent être d'une grande utilité (e.g., juges, policiers, douaniers, etc.). Ils ont trouvé une moyenne de décodage exact autour de 50\% - c'est-à-dire au niveau attendu pour des jugements effectués au hasard.

Sur la base de ces résultats, on pourrait soutenir que les différences objectives entre les expressions spontanées et simulées sont tout simplement trop faibles ou rapides pour être détectées. Toutefois, Ekman et O'Sullivan (1991) a trouvé des taux de réussite beaucoup plus élevés pour deux groupes d'individus, soit les agents de la CIA comptant entre 5 et I 5 années d'expérience et les enfants, surtout ceux provenant de familles abusives. Ces résultats suggèrent que (a) les expressions émotionnelles spontanées et intentionnelles diffèrent de manière objective, (b) ces différences sont observables en principe et (c) ces différences ne sont généralement pas observées ou interprétées.

Nous avons étudié davantage ce phénomène (Hess \& Kleck, 1990, 1994). Dans une première étude, nous avons demandé à des sujets de faire plusieurs tâches qui étaient construites de façon à provoquer des expressions faciales spontanées ou posées de joie ou de dégoût. En se basant sur le 
FACS (Ekman \& Friesen, 1978), le temps du début, le temps de la fin, et le temps à l'apex (sommet) furent observés pour chaque épisode. Puisqu'une seule expression peut avoir plusieurs débuts et fins, le nombre de phases fut aussi mesuré. Les résultats démontrent que la régularité des expressions volontaires et spontanées varie. Les expressions spontanées sont relativement coulantes et régulières, tandis que l'intensité des expressions volontaires démontre plus de pauses et de changements en escalier.

Dans une seconde étude, les sujets devaient regarder 60 expressions de joie et de dégoût et tenter de déterminer pour chaque vignette si l'expression était spontanée ou volontaire. À la suite de l'expérience, les sujets devaient rapporter les indices particuliers qu'ils avaient utilisés pour distinguer les deux types d'expressions. Ces indices, de même que les signaux qui avaient auparavant été identifiés comme étant des indicateurs valides, ont été mesurés objectivement.

Des modèles par équations structurales ont été utilisés afin de vérifier si (a) les scores de réussite des sujets étaient liés aux indicateurs qu'ils ont dit utiliser et (b) si ces indicateurs étaient valides. Les résultats ont démontré que (a) les sujets ont rapporté adéquatement les indices qu'ils ont employés pour prendre leur décision et (b) la majorité de ces indicateurs n'aident pas à discriminer de manière valide les expressions spontanées des expressions volontaires. Ainsi, les sujets n'ont pas pu bien faire la différence entre les expressions spontanées et volontaires et ce piètre niveau de succès vient de ce qu'ils ont constamment employé des indicateurs non valables.

\section{Le rôle du décodeur}

Les données mentionnées ci-haut suggèrent que la majorité des gens ont des croyances erronées concernant les signaux qui permettent de distinguer les deux types d'expressions. Ceci est assez étonnant si l'on constate que deux catégories de sujets étudiés par Ekman et O'Sullivan (|99|) ont été capables de faire cette distinction.

Par conséquent, comme l'a suggéré Kraut (1980), nous devons donc supposer que les adultes ne sont généralement pas motivés à détecter les expressions faciales fausses. Ce chercheur affirme que des interactions harmonieuses dépendent de la volonté des gens d'accepter les messages au pied de la lettre. Cette proposition est appuyée par un phénomène 
souvent observé: les individus ont tendance à considérer la majorité des messages comme étant véridiques (Zuckerman, DePaulo, \& Rosenthal, 1981). De plus, d'autres résultats indiquent que des sujets souffrant d'un niveau élevé d'anxiété sociale sont beaucoup moins habiles à détecter la tromperie que des sujets qui ont des faibles niveaux d'anxiété sociale (DePaulo \& Tang, 1994).

II se peut donc que les observateurs utilisent des «normes de décodage» pour guider leur interprétation des expressions faciales émotionnelles. Ces règles permettent à l'individu d'ignorer les signes qui rendent possibles l'identification des expressions volontaires. Ces normes de décodage incorporent les connaissances sociales sur l'exactitude de certaines expressions faciales, tout comme le font les lois d'affichage. En fait, les normes de décodage peuvent simplement être considérées comme l'envers des lois d'affichage. Ainsi, l'encodeur utilise les lois d'affichage pour modifier ses expressions. II se fait aider par l'observateur qui a tendance à décoder l'émotion appropriée plutôt qu'une autre.

Certains résultats trouvés dans notre laboratoire ont appuyé l'existence de ces normes d'affichage et de décodage par rapport aux expressions faciales d'émotions chez les hommes et les femmes. Pendant l'expérience, les sujets ont regardé 24 paires de dessins illustrant des expressions faciales émotionnelles. Chaque expression était associée à un profil facial féminin ou masculin. Les données indiquent que, pour la colère et la tristesse, la même expression émotionnelle associée à un profil féminin était jugée comme étant plus intense que pour les profils masculins. Pour la joie, le contraire s'est produit : les expressions de joie associées à un profil masculin étaient jugées comme étant plus intenses que pour les profils féminins. Ces résultats démontrent l'influence des connaissances des rôles sociaux sur le décodage. Vu qu'une femme est censée exprimer la colère soit moins fortement qu'un

124 homme, soit d'une manière différente (en pleurant), une même expression de colère est interprétée comme indiquant une colère moyenne chez un homme, mais une colère forte chez une femme.

\section{Conclusion}

En conclusion, la notion d'émotion simulée devrait être remplacée par la notion de l'affichage émotionnel simulé. La simulation des émotions peut se situer dans un contexte de tromperie. Par ailleurs, les règles sociales 
gouvernant les interactions de tous les jours demandent l'affichage de certaines émotions dans certaines situations. La prévalence de ces demandes ainsi que leur importance sont soulignées par le fait que même les enfants en bas âge savent s'y conformer. Dans une perspective qui accorde au décodeur un rôle actif dans les processus interactionnels, le faible taux de réussite dans le décodage des expressions simulées est interprété comme résultant d'un partage de ces règles sociales.

\section{RÉFÉRENCES}

Bavelas, J. B., Black, A., Lemery, C. R., \& Mullett, J. (1986). "I show how you feel": Motor mimicry as a communicative act. Journal of Personality and Social Psychology, 50, 322-329.

Buck, R. (1991). Social functions in facial display and communication: A reply to Chovil and others. Journal of Nonverbal Behavior, 15, I55-161.

Buck, R., Loslow, J. I., Murphy, M. M., \& Costanzo, P. (1992). Social facilitation and inhibition of emotional expression and communication. Journal of Personality and Social Psychology, 63, 962-968.

Bugenthal, D. B. (1986). Unmasking the "polite smile": Situational and personal determinants of managed affect in adult-child interaction. Personality and Social Psychology Bulletin, 12 , 7-16.

Cacioppo, J.T., Bush, L. K., \& Tassinary, L. G. (1992). Microexpressive facial actions as a function of affective stimuli: Replication and extension. Personality and Social Psychology Bulletin, $18,515-526$.

Chevalier-Skolnikoff, S. (1973). Facial expression of emotion in nonhuman primates. In P. Ekman (Ed.), Darwin and facial expression (pp. I I-83). New York, NY: Academic Press.

Chovil, N., \& Fridlund, A. J. (1991). Why emotionality cannot equal sociality: A reply to Buck. Journal of Nonverbal Behavior, 15, 163-167.

Cole, P. M. (1986). Children's spontanous control of facial expressions. Child Development, 57, 1309-1321.

Darwin, C. (1872/1965). The expression of the emotions in man and animals. Chicago: The University of Chicago Press. (Originally published, 1872).

DePaulo, B. M., \& Tang, J. (1994). Social anxiety and social judgement: The example of detecting deception. Journal of Research in Personality, 28, 142-153.

Ekman, P. (1979). About brows: Emotional and conversational signs. In K. F. M. von Cranach W. Lepenies, \& D. Ploog (Ed.), Human ethology (pp. 169-249). Cambridge: Cambridge University Press.

Ekman, P. (1984). Expression and the nature of emotion. In K. R. Scherer \& P. Ekman (Eds.), Approaches to emotion (pp. 319-344). Hillsdale, NJ: Erlbaum.

Ekman, P. (1992). An argument for basic emotions. Cognition and Emotion, 6, 169-200.

Ekman, P., Davidson, R. J., \& Friesen, W.V. (1990). The duchenne smile: Emotional expression and brain physiology II. Journal of Personality and Social Psychology, 58, 342-353.

Ekman, P.. \& Friesen, W.V. (197I). Constants across cultures in the face and emotion. Journal of Personality and Social Psychology, 17, 124-129. 
Ekman, P. \&. F., W.V. (1978). The Facial Action Coding System: A technique for the measurement of facial movement. Palo Alto, CA: Consulting Psychologists Press.

Ekman, P., \& Friesen, W.V. (1982). Felt, false, and miserable smiles. Journal of Nonverbal Behavior, 6. 238-252.

Ekman, P., Friesen, W. V., \& O'Sullivan, M. (1988). Smiles when lying. Journal of Personality and Social Psychology, 54, 4I4-420.

Ekman, P., \& O'Sullivan, M. (1991). Who can catch a liar? American Psychologist, 46, 913-920.

Fischer, A. (1993). Sex differences in emotionality: Fact or Stereotype? Feminism \& Psychology, 3, 303-318.

Fridlund, A. J. (|99|). The sociality of solitary smiling: Potentiation by an implicit audience. Journal of Personality and Social Psychology, 60, 229-240.

Fridlund, A. J., Kenworthy, K. G., \& Jaffey, A. K. (1992). Audience effects in affective imagery: Replication and extension to dysphoric imagery. Journal of Nonverbal Behavior, 16 (3), 191 -211 .

Frijda, N. (1986). The emotions. Cambridge: Cambridge University Press.

Gazzaniga, M. S., \& Smylie, C. S. (1990). Hemispheric mechanisms controlling voluntary and spontaneous facial expressions. Journal of Cognitive Neuroscience, 2, 239-245.

Hager, J. C., \& Ekman, P. (1985). The asymmetry of facial actions is inconsistent with models of hemispheric specialization. Psychophysiology, 22, 307-317.

Hess, U., Kappas, A., \& Banse, R. (1995). The intensity of facial expressions is determined by underlying affective state and social situation. Journal of Personality and Social Psychology, 69, 280-288.

Hess, U., Kappas, A., McHugo, G. J., Kleck, R. E., \& Lanzetta, J. T. (1989). An analysis of the encoding and decoding of spontaneous and posed smiles: The use of facial electromyography. Journal of Nonverbal Behavior, 13(2), 121-137.

Hess, U., \& Kleck, R. E. (1990). Differentiating emotion elicited and deliberate emotional facial expressions. European Journal of Social Psychology, 20, 369-385.

Hess, U., \& Kleck, R. E. (1994). The cues decoders use in attempting to differentiate emotion elicited and posed facial expressions. European Journal of Social Psychology, 24, 367-38I.

Hochschild, A. (1983). The managed heart. Berkely: University of California Press.

Hochschild, A. R. (1979). Emotion work, feeling rules, and social structure. American journal of Sociology, 85(3), 55I-573.

Izard, C. E. (197|). Human emotions. New York: Plenum.

Izard, C. E. (1990). Facial expressions and the regulation of emotions. Journal of Personality and Social Psychology, 58, 487-498.

126 Izard, C. E. (1991). The psychology of emotions. New York: Plenum,

Kraut, R. E. (1980). Humans as lie detectors: some second thoughts. Journal of Communication, 30, 209-216.

Lazarus, R. R. (199|). Emotion and adaptation. New York: Oxford University Press.

Lazarus, R. S. (1995). Vexing research problems inherent in cognitive mediational theories of emotion - and some solutions. Psychological Inquiry, 6, 183-196.

Markus, H. R., \& Kitayama, S. (1994). The cultural construction of self and emotion: Implications for social behavior. In S. Kitayama \& H. R. Markus (Eds.), Emotion and culture: Empirical studies of mutual influence Washington, DC: American Psychological Association.

Motley, M. T. (1993). Facial affect and verbal context in conversation: Facial expression as interjection. Human Communication Research, 20, 3-40. 
Plutchik, R. (1962). The emotions: Facts, theories and a new model. New York, NY: Random House.

Plutchik, R. (1980). Emotion: A psychoevolutionary synthesis. New York, NY: Harper \& Row.

Reissland, N., \& Harris, P. (199I). Children's use of display rules in pride-eliciting situations. British Journal of Developmental Psychology, 9, 431-435.

Rinn, W. E. (1984). The neuropsychology of facial expression: A review of the neurological and psychological mechanisms for producing facial expressions. Psychological Bulletin, 95, 52-77.

Rinn, W. (1991). Neuropsychology of facial expression. In R. S. Feldman \& B. Rimé (Eds.), Fundamentals of nonverbal behavior (pp. 3-30). Cambridge: Cambridge University Press.

Saarni, C. (1979). Children's Understanding of display rules for expressive behavior. Developmental Psychology, 15, 424-429.

Scherer, K. R. (1986). Vocal affect expression: A review and a model for future research. Psych. Bull., 99(2), 143-165.

Tomkins, S. S. (1962). Affect, imagery, conciousness: The positive affects. New York: Springer.

Tomkins, S. S. (1963). Affect, imagery, conciousness: The negative affects. New York: Springer.

Tomkins, S. S. (199|). Affect, imagery, conciousness: Anger and fear. New York: Springer.

Tomkins, S. S. (1993). Affect, imagery, conciousness: Cognition - Duplication and transformation of information. New York: Springer.

Weiss, F., Blum, G.S., \& Gleberman, L. (1987). Anatomically based measurement of facial expressions in simulated versus hypnotically induced affect. Motivation and Emotion, II, 67-81.

Wierzbicka, A. (1994). Emotion, language, and cultural scripts. In S. Kitayama \& H. R. Markus (Eds.), Emotion and culture: Empirical studies of mutual influence Washington, DC: American Psychological Association.

Wierzbicka, A. (1995). The relevance of language to the study of emotions. Psychological Inquiry, 6, 248-252.

Wundt, W. (1903). Grundzüge der physiologischen Psychologie. Leipzig: Wilhelm Engelmann.

Zuckerman, M., DePaulo, B. M., \& \& Rosenthal, R. (198I). Verbal and nonverbal communication of deception. In L. Berkowitz (Ed.), Advances in experimental social psychology (pp. 1-59). New York: Academic Press. 
(Página deixada propositadamente em branco) 


\section{Arvid Kappas}

The University of Hull

Jean Descôteaux

Université Laval

\section{LES PROMESSES ET LIMITES DE L'ÉTUDE DE L'ÉMOTION EN LABORATOIRE}

\section{Introduction}

Lorsque Charles Darwin écrivait son ouvrage «The expression of the emotions in man and animals» (1872/1998), il a dû se fier en grande partie sur des observations récoltées en milieu naturel. Bien que certaines des recherches qu'il a citées, comme celle de Duchenne (I862/1990), aient été exécutées en laboratoire, la grande majorité d'entre elles reposaient sur des observations libres et anecdotiques. Depuis, inspirés par les idées de Darwin, les chercheurs ont tenté d'étudier systématiquement les processus émotionnels dans l'environnement contrôlé du laboratoire. En fait, la grande majorité des connaissances actuelles au sujet de la psychologie de l'émotion provient de la recherche en laboratoire, et l'avancement de ces connaissances repose en grande partie sur les succès de cette démarche.

Étant donné le rôle central qu'occupe la recherche en laboratoire, il importe de poser un regard critique sur les aspects méthodologiques des études qui supportent notre compréhension, qu'elles soient anciennes, récentes ou futures. Quelques-uns des plus grands désaccords qui séparent les théoriciens semblent d'ailleurs causés par certaines conditions et contraintes spécifiques au contexte de laboratoire (par exemple, la question de l'existence de patrons psychophysiologiques périphériques distincts pour certaines émotions, l'influence des facteurs sociaux sur les expressions faciales, ou l'opposition entre l'approche catégorielle voulant qu'il existe des émotions fondamentales et l'approche dimensionnelle qui en contredit l'existence). De plus, les choix méthodologiques peuvent influencer des domaines de recherche entiers. Par exemple, l'étude de l'influence des états émotifs sur les processus cognitifs dépend en grande partie des états affectifs qu'il est possible de créer en laboratoire. 
Au-delà de l'aspect critique, il faut encore apprécier la multitude des choix qui s'imposent aux chercheurs intéressés par l'étude des processus affectifs. En effet, on peut de moins en moins justifier l'ignorance des développements rapides qu'a connus la psychologie de l'émotion et l'utilisation de méthodes devenues inadéquates pour l'avancement de notre science.

Ce chapitre vise à clarifier certaines des difficultés que rencontrent les chercheurs qui veulent étudier les processus émotionnels en laboratoire. En premier lieu, nous aborderons la question de la production des émotions: peut-on susciter des émotions «réelles» en laboratoire, diffèrent-elles des émotions rencontrées dans la vie de tous les jours et, si oui, de quelle façon? Certaines émotions sont-elles plus faciles à étudier que d'autres et pourquoi? Nous explorerons ensuite la mesure de l'émotion : quelles options sont disponibles lorsqu'il s'agit de mesurer le comportement expressif, la réponse physiologique et l'expérience subjective? Quels en sont les problèmes, qu'ils soient de nature pratique ou conceptuelle?

\section{L'induction des émotions en laboratoire}

\section{I Pourquoi étudier les émotions en laboratoire?}

II est possible d'étudier l'émotion en cours de déploiement, qu'on la contrôle en laboratoire ou qu'on l'observe dans le «monde réel». Une méthode alternative fréquemment utilisée consiste à analyser les souvenirs d'épisodes émotionnels passés ou à faire l'exploration de situations imaginaires dans un cadre présent ou futur. Bien qu'il soit commode de demander à des participants de décrire certains de leurs épisodes émotionnels passés, que ce soit au moyen de questionnaires ou d'entrevues, cette procédure n'est pas exempte de problèmes. En effet, certains résultats récents suggèrent que ces «souvenirs» seraient en fait des constructions fondées à la fois sur les expériences réelles passées, sur certains schéma sociaux et personnels ainsi que sur une variété de processus cognitifs (voir le chapitre de Philippot dans ce volume). Cependant, bien que les productions des participants ne constituent pas un compte rendu objectivement exact des événements passés, il ne faut pas commettre l'erreur de les considérer comme des données biaisées et inutiles. II s'agit plutôt de les interpréter dans le cadre d'une approche hétérophénoménologique 
(Dennett, 1991). Dans ce contexte, il est possible d'interpréter la description que donne un participant des éléments de la situation qui ont précédé sa réaction émotionnelle et de l'évolution de cette réaction comme un produit de la structure cognitive de cet individu. II n'y a aucun moyen de distinguer les «faits» de ce qui est du ressort de «l'imagination» des participants puisque leurs comptes rendus sont «factuels» pour ce qui est de leur propre représentation subjective. Même dans les cas où il existe un degré de correspondance élevé entre les comptes rendus, on ne peut conclure qu'ils constituent des descriptions «valides» des situations «objectives» passées. Il est possible qu'ils soient plutôt représentatifs de certains processus partagés par plusieurs ou la majorité des individus d'un groupe ou d'une culture donnée (Rimé, Philippot, \& Cisamolo, 1990). En conséquence, nous devrions étudier les souvenirs et les épisodes fictifs dans le but avoué de comprendre les représentations cognitives, et surtout éviter de les considérer comme un moyen économique pour mesurer les processus émotionnels en cours, comme les réponses physiologiques. En effet, le choix de l'examen rétrospectif des situations émotionnelles devrait être justifié par des raisons théoriques et non pas par des aspects de commodité ! Par ailleurs, au-delà de la question de l'exactitude des expériences rapportées par les participants, il reste à savoir quels rôles jouent l'imagerie et les souvenirs d'événements passés au sein du processus émotionnel qui accompagne le rappel lui-même. Dans les deux cas, l'expérience a démontré que ces méthodes exercent un puissant effet sur l'expérience subjective, ainsi que sur les réponses physiologiques et expressives.

Dans les domaines étroitement liés à la psychologie, comme l'éthologie, l'anthropologie, ou la sociologie, le travail doit reposer par nécessité sur les méthodes d'observation en environnement naturel. Des contraintes pratiques et éthiques imposent l'utilisation de méthodes similaires dans certains domaines de la psychologie, comme la recherche clinique ou du développement. Bien entendu, quoique leur nécessité ait été démontrée, les méthodes fondées sur l'observation naturelle sont taxées d'une variété de problèmes. Puisque ces problèmes sont habituellement explorés à fond dans les manuels d'introduction ou même les textes plus avancés portant sur la méthodologie de la recherche, nous éviterons de reproduire ici ce qui représente un terrain connu pour la vaste majorité et nous concentrerons plutôt sur une discussion de la recherche des émotions «in vivo» en laboratoire. 
L'histoire de l'induction d'émotions en laboratoire couvre presque un siècle entier. Alors que certaines des méthodes utilisées dans les études classiques sont amusantes, d'autres sont parfois choquantes. Landis, par exemple, requérait de ses participants qu'ils décapitent des rats, qu'ils plongent leurs mains dans des contenants remplis de grenouilles vivantes, ou qu'ils soient les cibles innocentes d'explosions surprises de pétards sous leurs chaises (i.e., Landis, 1924a; Landis, 1924b; Landis \& Gullette, 1925). En fait, plusieurs des études classiques en psychologie des émotions sont davantage connues pour leurs méthodes d'induction que pour les détails des raisonnements théoriques de leurs auteurs ( $A x, 1953$; Schachter \& Singer, 1962). Aujourd'hui, au contraire, les chercheurs évitent d'avoir recours à ce genre de situation extrême. Cependant, il est difficile de savoir si leur choix est fondé sur leurs propres convictions ou sur celles des comités de déontologie des institutions où ils travaillent!

\subsection{Méthodes utilisées pour l'induction des émotions}

À ce jour, plusieurs méthodes ont été utilisées dans les laboratoires afin d'induire des émotions ou des changements d'humeur chez les participants. Quelques chercheurs ont tenté de forger des catégories afin de regrouper les multiples méthodes en sous-groupes relativement homogènes. Leurs catégories, par exemple, reposent sur le fait que les participants sont au courant ou non de la manipulation (Martin, 1990), qu'ils sont au courant ou non de l'objectif de l'induction d'émotion (Gerrads-Hesse, Spies, \& Hesse, 1994), ou bien que les paradigmes requièrent qu'ils interagissent avec quelqu'un d'autre par opposition à ce qu'ils complètent leur tâches de façon isolée. Dans les faits, plusieurs de ces méthodes utilisent des procédés qui sont éminemment similaires (i.e., présentation de diapositives) et appartiennent ainsi à la même catégorie, alors que les méthodes restantes sont plus inusitées et constituent les seules représentantes de leur catégorie. La liste suivante, bien que non exhaustive, présente une variété de techniques utilisées pour induire les émotions.

- Imagerie mentale, auto-génération, rappel de souvenirs à teneur émotive

- Affirmations faisant référence à soi, méthodes Velten et Velten modifiée 
- Hypnose

- Production dirigée d'expressions faciales, rétroaction en provenance du visage, rétroaction en provenance de la posture du corps

- Manipulation de l'information concernant la performance du participant (succès vs échec)

- Stimuli sensoriels/concrets (i.e. odeurs, chocs, bruits)

- Menace, anticipation

- Films, diapositives, photographies (incluant des représentations d'expressions émotives)

- Musique, poésie

- Entrevues visant à stresser les candidats, agissements des expérimentateurs/complices

- Jeux vidéo et simulations

- Contagion sociale, expression en public, processus emphatiques

- Interventions pharmacologiques/centrales (stimulation du cerveau)

Au fur et à mesure des progrès effectués dans le domaine de la technologie, les ordinateurs prennent un rôle de plus en plus important pour l'induction des émotions. Aujourd'hui, par exemple, on a couramment recours à l'ordinateur pour présenter des photographies ou des vidéos à l'écran. Demain, on l'utilisera sans aucun doute pour supporter les technologies apparentées à la réalité virtuelle. En effet, en postulant que les problèmes liés aux environnements synthétiques soient bientôt résolus (voir Rowe, 1995), l'utilisation de ces technologies sera justifiée en tant qu'elles (a) accroissent le réalisme des situations et ainsi leur pertinence pour les participants, (b) offrent, en théorie, l'avantage d'un contrôle absolu sur les paramètres des situations présentées, et (c) favorisent en même temps l'enregistrement en unités quasi infinitésimales des actions et réponses des participants. Certaines techniques de réalité virtuelle ont d'ailleurs déjà commencé à être utilisées dans le traitement clinique des phobies (voir Rothbaum, Hodges \& Kooper, 1997).

\subsection{Efficacité des techniques d'induction des émotions}

À ce jour, la plupart des revues effectuées quant aux méthodes d'induction des émotions n'ont porté que sur un nombre restreint d'études. 
De plus, lorsque les chercheurs ont rapporté des scores d'efficacité, ceux-ci n'ont été présentés que sous forme de tableaux descriptifs (e.g., GerradsHesse et al., 1994). Néanmoins, dans la revue qu'il a effectuée récemment, Sinnett a eu recours à des techniques de méta-analyse pour comparer les tailles d'effet des différentes méthodes recensées et ce, en fonction d'une variété de critères. Ils ont calculé, à partir de 225 articles publiés, une taille d'effet moyenne de $d=.69$ (à partir de 89/ effets; données non publiées, Sinnet 1995). Par ailleurs, et de façon surprenante, ils n'ont noté que très peu de différences systématiques entre les sexes (Sinnett, 1995). Westermann, Spies, Stahl et Hesse (1996) ont également comparé l'efficacité des méthodes d'induction à partir d'un échantillon plus restreint de | | | articles. Les résultats de leur méta-analyse montrent que l'induction d'émotions à partir de films avec l'instruction d'essayer d'atteindre un état subjectif spécifique est la plus efficace.

Les différentes méthodes d'induction d'émotions ne varient pas seulement en fonction de leur efficacité, mais aussi en fonction d'autres dimensions dont il faut tenir compte lors du choix de la méthode la plus appropriée pour une question de recherche donnée (voir aussi Kappas, 1989; Larsen \& Sinnet, 1991; Martin, 1990). La liste suivante présente certaines de ces dimensions importantes:

- Taux de succès

- Spécificité (à quel point les états affectifs induits sont-ils purs)

- Homogénéité: différences inter individus (la méthode ou le stimulus induit-il le même état chez différents individus?)

- Restrictions des groupes (en fonction de l'âge, du sexe/genre, de la culture, du tableau clinique)

- Flexibilité de la méthode (tous les états affectifs peuvent-ils être induits suivant cette méthode, leur intensité peut-elle être manipulée?)

- Aspects temporels (court/long, variable/constant) - incluant le temps d'administration

- La méthode peut-elle être utilisée à répétition? - Combien de fois?

- La méthode est-elle compatible avec une administration de groupe?

- Compatibilité avec les variables dépendantes

- Coûts (entraînement, requis technologiques/personnels) 
- Susceptibilité à l'effet des attentes de la part des expérimentateurs

- Considérations éthiques

À l'aide de ces dimensions, il serait possible de créer un espace multidimensionnel sur lequel on placerait un point pour représenter chaque méthode. À ce jour, personne n'a entrepris de réaliser une telle tâche (voir aussi Martin, 1990). Certaines raisons bien précises peuvent être invoquées pour justifier cette apparente inertie : il n'existe que très peu de données normatives relatives aux différentes méthodes, de même qu'il soit possible qu'un effort de ce genre demeure fondamentalement improductif - il est évident que la question de déterminer quelle est la meilleure méthode reste discutable, puisque le choix ne peut que dépendre de la question de recherche. Partant, il devient préférable, au lieu d'adopter une seule méthode et de l'utiliser à toutes les sauces, de se construire une «boîte à outils» contenant une variété de méthodes différentes, et de retenir celles qui semblent les plus adaptées aux besoins immédiats. D'ailleurs, l'utilisation de plusieurs méthodes d'induction différentes tend à renforcer les résultats, puisqu'elle réduit la possibilités que ceux-ci ne constituent que des artefacts liés à telle ou telle méthode spécifique (par exemple, certains changements comportementaux et physiologiques découlent directement de l'utilisation de l'hypnose, n'ayant ainsi rien à voir avec l'émotion induite chez le participant).

Récemment, certains chercheurs ont tenté de construire des ensembles standardisés de stimuli émotionnels (voir Gross \& Levenson, 1995) afin de faciliter le processus d'induction. Cependant, les résultats de ces efforts demeurent controversés, puisque la question de l'universalité des stimuli n'a pas été entièrement résolue par les théoriciens. Par ailleurs, on doit se rappeler que le but principal de la psychologie de l'émotion ou même de la psychologie cognitive n'est pas de développer des stimuli capables d'induire des émotions, mais bien d'étudier les réactions émotives et cognitives des participants. Partant, n'importe quelle technique qui fonctionne et qui respecte les critères déontologiques peut être utilisée. À l'inverse, si telle étude ne présente aucune évidence que les états émotifs recherchés ont bel et bien été induits chez les participants, il n'y a aucune raison valable d'en accepter les résultats. Toutefois, le choix des critères qui contribuent à déterminer ce qui constitue une vérification valable demande certainement à être approfondi (voir ci-après). 


\subsection{Les théories de l'émotion actuelles peuvent-elles aider à résoudre les problèmes associés à l'induction des émotions en laboratoire?}

Lorsque nous induisons des émotions en laboratoire, nous nous attendons à ce que les processus activés chez les participants soient similaires à ceux rencontrés dans «le monde réel à l'extérieur du laboratoire». Or, comment se fait-il que les chercheurs oublient leurs théories dès qu'ils se mettent à discuter des questions de laboratoire? Par exemple, pour des raisons déontologiques, les méthodes d'induction utilisées aujourd'hui sont «douces»; plusieurs chercheurs se plaignent de cet état de choses, et affirment que l'absence de résultats robustes (par exemple dans le domaine de la psychophysiologie) est causée par la faible puissance des stimuli inducteurs d'émotions utilisés. Cette plainte paraît être valide en surface, mais ce qui surprend, c'est bien l'absence du facteur puissance ou intensité des stimuli dans les théories de l'émotion actuelles.

À l'inverse, il est remarquable de constater à quel point certains éléments théoriques importants peuvent être complètement ignorés dans la pratique. Par exemple, la plupart des théories modernes, et plus particulièrement les théories cognitives de l'émotion (voir Scherer \& Sangsue, ce volume; Scherer, Schorr, \& Johnstone, 200I), accordent une grande importance à la pertinence qu'un stimulus ou événement revêt pour les plans, buts et besoins d'un individu. Ainsi, un participant ne sera stressé par une tâche mathématique difficile que s'il est important et pertinent pour lui de réussir cette tâche. L'efficacité des méthodes apparentées à la technique Velten (Velten 1968), fondée par définition sur des affirmations faisant référence à soi, respecte justement cette façon de voir (mais voir Buchwald, Strack, \& Coyne, 1981; Westermann et al., 1996). La psychologie sociale a suivi une direction similaire en s'efforçant d'accroître la pertinence de ses tâches expérimentales. Par exemple, les participants qui doivent juger les caractéristiques d'une personne sont informés à l'avance qu'ils devront interagir avec celle-ci une fois la tâche terminée. À tout prendre, il semble donc logique de penser que les émotions puissent être évoquées avec plus de facilité lorsque les stimuli ou événements sont pertinents pour le participant. II importe pourtant de noter que le facteur de pertinence ne représente qu'une des nombreuses facettes des recherches en laboratoire qui pourraient être améliorées par une application plus rigoureuse des théories de l'émotion. 
Partant des théories de l'émotion, il est aussi frappant de constater qu'aucune des trois grandes familles de théories actuelles n'offre de raisons a priori permettant de remettre en question l'utilisation de la recherche en laboratoire. L'approche des émotions fondamentales (e.g., Tomkins, Izard, Ekman) postule l'existence de programmes neuronaux innés spécifiques à chacune de ces émotions. Lorsque ces programmes sont activés, ils génèrent des signaux qui sont acheminés à travers une série de réseaux innés («hard-wired»), dont l'effet est de créer des changements temporaires au sein des sphères physiologique, expressive, et de l'expérience subjective. Ce genre de postulat implique qu'il ne doit exister aucune différence, hormis peut-être d'intensité, entre la colère naturelle et la colère induite en laboratoire. Ainsi, les chercheurs réunis par cette façon de voir ne devraient s'attendre qu'à des variations quantitatives lorsqu'ils comparent les émotions générées dans les deux contextes. De leur côté, les adeptes de l'approche de l'évaluation cognitive des stimuli émotionnels («appraisal»; e.g., Arnold, Lazarus, Scherer, Smith, Roseman; voir aussi chapitre de Scherer \& Sangsue) chercheraient à comparer, comme explicité précédemment, le degré de pertinence accordé par les participants aux situations naturelles et de laboratoire. Bien que ce point de vue les distingue des tenants de l'approche des émotions fondamentales, ils considèrent néanmoins qu'une série d'évaluations cognitives donnée entraîne les mêmes changements aux plans physiologique, expressif et de l'expérience subjective, que ceux-ci découlent d'un jeu vidéo utilisé en laboratoire (i.e., Kappas \& Pecchinenda, 1999) ou d'une situation naturelle. Finalement, les tenants de l'approche dimensionnelle (e.g., Lang, Russell; voir Kirouac, 1995) pourraient défendre l'idée que les paradigmes de laboratoire génèrent des degrés d'activation différents de ceux créés par les situations naturelles, mais eux aussi postuleraient l'absence de différences qualitatives entre les deux contextes (voir aussi Kappas, sous presse-a).

De façon évidente, l'ensemble des chercheurs en émotion aimerait pouvoir repousser les limites de chacune des méthodes d'induction existantes, abolissant ainsi la nécessité de recourir à plusieurs méthodes différentes. Dans un monde idéal, ils disposeraient d'un seul ensemble de stimuli générateurs d'émotions applicable dans diverses cultures, dans une culture donnée comprenant plusieurs sous-cultures, et de façon répétée auprès des mêmes participants (voir précédemment). Le «International Affective Picture System» (Center for the Study of Emotion and Attention 
[Csea], 1994; mais voir aussi Gross \& Levenson, 1995; Philippot, 1993) représente l'effort le plus important investi récemment dans cette direction. Le IAPS comprend maintenant environ 900 diapositives ayant été testées dans plusieurs cultures (voir Hamm \& Vaitl, 1989) qui semblent générer partout les mêmes rapports subjectifs (habituellement mesurés à l'aide de deux ou trois échelles dimensionnelles, par opposition à une série d'étiquettes émotionnelles discrètes) ainsi que certaines réponses physiologiques (voir aussi Lang, Greenwald, Bradley, \& Hamm, 1993).

On est en droit de se demander comment ces diapositives peuvent engendrer des réactions affectives spécifiques invariables en fonction des cultures. La série de diapositives du IAPS comprend, en fait, des images de bébés et d'animaux, de même que des photographies érotiques, qui ont apparemment le pouvoir de susciter directement l'intérêt de celui qui les regarde. Or, suivant le postulat que les processus affectifs ne se limitent pas aux processus conscients (i.e., Scherer, 1993), ces images font probablement appel à un niveau de traitement schématique (mais pas nécessairement de façon exclusive) plutôt que conceptuel (Leventhal \& Scherer, 1987; Scherer, 200 I). Partant, elles constituent de bons candidats pour ce qui est des présentations subliminales (voir Öhman, 1993) et des expériences de conditionnement et de modulation des réflexes (e.g., Bonnet, Bradley, Lang, $\&$ Requin, 1995). Cependant, elles ne représentent pas adéquatement les processus affectifs qui découlent de l'évaluation continue des événements que font les individus en fonction de leurs buts et besoins. De même, par leur aspect statique, elles ne permettent pas l'étude des caractéristiques temporelles (voir Tomkins, 1962, 1963) des stimuli inducteurs d'émotions (Kappas, Hess, \& Banse, 1992). Bref, l'image est un bon véhicule pour la présentation de stimuli calmants ou stimulants, mais puisqu'elle manque souvent de pertinence vis-à-vis des buts et besoins de l'observateur, elle ne peut contribuer à l'induction de certains états émotionnels comme la colère. Qu'arrive-t-il alors du cas des émotions qui nécessitent un traitement cognitif plus profond que celui requis par le traitement de diapositives?

Voilà justement pourquoi plusieurs études font appel à des films ou à des textes écrits pour induire les états affectifs. Ces stimuli, qui peuvent reproduire des événements réels ou en présenter d'autres créés de toutes pièces, sont reconnus pour la puissance des réactions affectives qu'ils induisent chez ceux qui les regardent. Certains auteurs vont même jusqu'à affirmer qu'ils peuvent induire fidèlement des états affectifs spécifiques (e.g., 
Gross \& Levenson, 1995; McHugo, Smith, \& Lanzetta, 1982; Philippot, 1993). On reconnaît aisément pour soi la puissance dont dispose ce type de stimuli : en effet, nous payons volontiers une somme rondelette pour assister à des présentations de pièces de théâtre et de films sur grand écran dans l'espoir d'être émus, voire complètement envahis par les émotions. De plus, nous passons de plus en plus de temps à regarder les émissions de télévision et notre expérience nous dicte que même le petit écran peut induire en nous des émotions fort intenses. Néanmoins, il est improbable que nous partagions tous les mêmes réactions lorsque nous regardons ces émissions. Les réactions à ces stimuli dépendent habituellement des connaissances, croyances et systèmes de valeurs propres à un groupe social donné, une culture, une sous-culture ou même un individu en particulier. Par exemple, certains films, textes ou photographies qui présentent ou décrivent des gestes horribles posés lors de guerres historiques peuvent induire de la colère ou de la haine chez un individu, du dégoût et du mépris chez un autre, et de la culpabilité et de la tristesse chez un troisième. La présentation d'un documentaire à propos de la guerre en Bosnie ou en Afghanistan peut non seulement induire des émotions différentes en fonction des connaissances, croyances et valeurs, mais aussi en fonction du moment de l'émission, que ce soit par exemple maintenant, ou dans dix ou vingt ans. Lorsqu'on y regarde de plus près, même les émotions qu'on croit être capable d'induire facilement chez les gens, comme l'amusement par exemple, ne sont pas si simplement évoquées. II est en effet rare de trouver un comique qui fasse rire la majorité des gens. En fait, il n'existe à l'heure actuelle aucun ensemble de stimuli capable d'induire fidèlement les mêmes réactions indépendamment des cultures, genres, âges, etc. Nous croyons d'ailleurs qu'il n'en existera jamais, simplement à partir de notre compréhension de ce qui déclenche les réactions émotionnelles! De plus, nous pensons qu'il est erroné de tirer des conclusions quant à la structure de l'émotion à partir de résultats provenant d'une méthode particulière qui demeure limitée quant aux émotions qu'elle peut induire.

Lorsque nous étudions le processus d'induction d'émotion en laboratoire, nous devons non seulement tenir compte des stimuli et méthodes d'induction utilisées, mais aussi du contexte dans lequel se retrouvent les participants. Ekman et Friesen (1969) ont affirmé que les expressions ne reflètent souvent pas l'émotion sous-jacente véritable, que ce soit au plan quantitatif ou qualitatif. Ils ont proposé l'existence de règles de gestion de 
l'expression («display rules»), qui consistent en des normes culturellement partagées qui spécifient ce qu'il est approprié de montrer et à qui il est permis de le faire. Influencés par l'idée que le contexte social peut exercer une forte influence sur les expressions émotives, plusieurs chercheurs ont alors décidé d'isoler leurs participants lors du processus d'induction, allant parfois même jusqu'à ne pas les informer qu'ils étaient observés.

En isolant les participants dans une chambre, les privant ainsi de tout contact avec d'autres personnes, ils croyaient contrer l'effet potentiel des règles culturelles et sociales qui gèrent l'expression. Toutefois, Fridlund (199|) a pu montrer que le fait de savoir ou non qu'un ami, situé dans une salle adjacente, exécute soit la même tâche, soit une tâche différente, influence les expressions montrées par les participants. En d'autres termes, l'isolement physique n'élimine pas l'effet du contexte social. Comme l'a dit Fridlund, nous destinons continuellement nos expressions aux personnes que nous avons en tête. L'étude de Fridlund a pu être reproduite et même enrichie, les résultats montrant alors que l'émotion sous-jacente et le contexte social implicite influencent tous deux le comportement expressif des participants testés en isolation (Hess, Banse, \& Kappas, 1995). Les conséquences immédiates de ces études et d'autres similaires sont (a) que nous devons porter une grande attention à l'effet de la présence d'autrui sur les participants, qu'il s'agisse d'expérimentateurs ou d'autres participants, (b) que nous devons jauger avec précaution l'utilisation de caméras visibles, de fenêtres et miroirs d'observation, et (c) qu'il est impossible de présumer qu'un participant, exécutant une tâche, seul dans le local d'expérimentation, ne subit pas l'influence du contexte social.

II nous reste encore à souligner la présence d'une importante confusion théorique qui perturbe l'avancement de la recherche en émotion, soit la distinction entre humeur et émotion. On convient qu'elles appartiennent toutes deux à la catégorie des processus affectifs, mais qu'elles se distinguent l'une de l'autre selon leurs caractéristiques temporelles et le lien qu'elles entretiennent avec des stimuli spécifiques (Ekman, 1984; Kirouac, 1995). Or, certaines études font appel aux méthodes propres à influencer l'humeur des participants, alors que d'autres utilisent des techniques qui permettent d'induire des émotions (toutes deux se retrouvent au sein des méthodes décrites précédemment). II y a là un réel problème puisque les publications qui passent en revue les résultats d'études n'ont généralement pas fait la distinction et les ont regroupés ensemble. Bien que la frontière exacte entre 
humeur et émotion puisse être parfois difficile à préciser, nous devons accepter qu'il s'agit de deux entités distinctes et ne pouvons nous permettre d'ignorer leur caractère distinct pour des raisons de commodité.

Nous avons choisi de ne pas aborder l'importante question relative aux aspects déontologiques de la recherche en émotion. Bien que les règlements locaux puissent influencer les chercheurs, la pression que les revues scientifiques exercent sur eux pour qu'ils respectent les standards de «l'American Psychological Association», ou de toute autre association scientifique ou professionnelle, quant au traitement des participants est de plus en plus forte. L'espace limité dont nous disposons ici ne nous permet pas d'aborder les aspects philosophiques et pratiques de ces règlements. Nous nous bornerons à souligner que l'auto-restriction des chercheurs œuvrant dans le domaine des processus émotionnels risque de limiter l'étendue des phénomènes observés. Non pas que nous désirions ici cautionner l'utilisation de méthodes d'induction extrémistes et non déontologiques. II s'agit bien de souligner le danger inhérent à l'interprétation des phénomènes étudiés en laboratoire comme étant représentatifs de la totalité des processus existants et à la formulation de conclusions fortes étayées sur ce genre d'hypothèse.

\section{La mesure des processus émotionnels en laboratoire}

De plus en plus de chercheurs en émotion s'accordent aujourd'hui pour dire que le processus émotionnel est constitué de plusieurs composantes : l'expérience subjective, la réponse physiologique et les réactions expressive et comportementale. Ces multiples facettes représentent autant de façons de mesurer l'émotion. Les rapports subjectifs des participants ont largement été utilisés au cours de l'histoire de la recherche en émotion. Certains chercheurs d'avant-garde ont également eu très tôt recours à des mesures psychophysiologiques, expressives ou comportementales sophistiquées. Aujourd'hui, des ordinateurs de plus en plus puissants ouvrent de nouvelles voies aux chercheurs qui veulent mesurer les processus émotionnels. L'espace dont nous disposons ne nous permet de présenter qu'une poignée de méthodes et technologies essentielles à la mesure du comportement non verbal et des réactions psychophysiologiques, ainsi qu'une brève discussion des problèmes généraux liés à la mesure en émotion. 


\section{I Comportement non verbal}

Les expressions faciales étaient importantes pour Charles Darwin; elles continuent de l'être aujourd'hui pour plusieurs chercheurs qui ont choisi d'en faire leur objet d'étude. Les expressions faciales, en tant que comportements non verbaux, peuvent être étudiées chez des êtres qui ne parlent pas le même langage que nous ou qui n'ont simplement pas encore développé cette capacité. De plus, certains croient qu'elles peuvent révéler chez un individu la présence d'émotions qu'il cherche à contrôler ou à cacher sous la pression de demandes sociales ou circonstancielles. À ses débuts, la recherche dans le domaine des expressions faciales a été entravée non par une lacune au plan technologique, mais par l'absence d'une terminologie et de standards communs. Paul Ekman et Wallace Friesen ont publié en 1978 ce que d'aucuns considèrent être le système objectif le plus puissant pour mesurer les mouvements faciaux : le «Facial Action Coding System (FACS)» (Ekman \& Friesen, 1978; voir aussi Sayette, Cohn, Wertz, Perrott, \& Parrott, 200 I). Au sein de cette méthode, toutes les descriptions sont effectuées à partir de données anatomiques et comportementales. Les codificateurs FACS, qui subissent un entraînement standardisé, doivent passer un test avant d'être reconnus officiellement. Cette procédure permet d'établir le type de fidélité interjuges dont a grandement besoin ce domaine de recherche. Cependant, plusieurs chercheurs évitent d'utiliser le FACS à cause de l'importance du temps requis pour l'entraînement des codificateurs ainsi que pour l'analyse des comportements faciaux en tant que tels - le temps d'analyse prend souvent cent fois la durée de l'épisode à codifier. Certains, et plus particulièrement les chercheurs en psychologie du développement (voir aussi Oster, Hegley, \& Nagel, 1992), lui préfèrent un système développé par Izard, le MAX (Izard, 1979), un instrument similaire mais moins détaillé. Ceux qui

142 s'intéressent à des émotions spécifiques utilisent parfois des versions abrégées de ces systèmes, comme l'AFFEX et l'EMFACS, qui soi-disant permettent la codification en temps réel des extraits. Récemment, la codification assistée par ordinateur a gagné en puissance et est également devenue plus abordable (Kappas, 1992; Noldus, Van de Loo, \& Timmers, 1989; Noldus, Trienes, Hendricksen, Jansen, \& Jansen, 2000). Cette technologie permet aux codificateurs d'interagir avec un programme informatique qui inscrit directement les codes dans l'ordinateur et gère le fonctionnement de l'appareil vidéo. Le nombre d'erreurs et le temps requis pour la 
codification s'en trouvent diminués, ce qui augmente l'efficacité de l'ensemble du processus.

L'électromyographie (EMG) faciale est une autre méthode de mesure dont la popularité s'accroît rapidement. L'EMG permet d'enregistrer l'activité des muscles faciaux si faible qu'elle ne pourrait être détectée à l'œil nu, et sa résolution temporelle surpasse de plusieurs ordres de grandeur celle des méthodes vidéo. La recherche a démontré que l'EMG est non seulement capable de distinguer les émotions négatives des positives (Cacioppo, Petty, Losch, \& Kim, 1986), mais peut aussi discriminer une variété d'émotions négatives (Hess, Kappas, McHugo, Lanzetta, \& Kleck, 1992; Vrana, 1993) et distinguer les expressions volontaires des expressions spontanées (Hess, Kappas, McHugo, Kleck, \& Lanzetta, 1989; Hess \& Kleck, 1990). Autre avantage, des standards concernant l'enregistrement de l'EMG faciale ont été développés et publiés et sont donc accessibles aux chercheurs intéressés (Fridlund \& Cacioppo, 1986). Les coûts jadis prohibitifs et la rareté des équipements d'hier ne représentent plus un réel problème aujourd'hui, le prix des appareils et des programmes informatiques ayant chuté et plusieurs compagnies offrant des systèmes dont l'adaptation demeure simple en vertu de l'utilisation pratique de l'EMG. Cependant, contrairement aux méthodes visuelles, l'EMG nécessite l'application d'électrodes sur la peau du visage, ce qui peut interférer avec le déroulement normal des tâches (Kleck et al., 1976). De plus, des niveaux élevés d'activité faciale peuvent entraîner des interférences entre les différents sites musculaires mesurés. Comme indiqué précédemment (i.e., Tassinary, Cacioppo, \& Geen, 1989), l'EMG et le FACS ne sont pas des méthodes rivales, mais sont bel et bien complémentaires.

L'apparition récente de systèmes informatiques capables de codifier automatiquement l'activité du visage à partir d'images visuelles représente peut-être l'innovation la plus excitante à l'heure actuelle pour la recherche en émotion (e.g., Kaiser \& Wehrle, 1992, 200 I; Yamada, Chiba, Tsuda, Maiya, \& Harashima, 1992; Cohn, Zlochower, Lien, \& Kanade, 1999). En fait, la plupart de ces systèmes n'ont pas été créés pour l'étude spécifique des expressions faciales liées à l'émotion, mais pour développer, par exemple, de nouveaux moyens de télécommunication ou pour démontrer la puissance des modèles qui reposent sur les réseaux neuronaux. Néanmoins, il est clair que ce genre de système, une fois mis sur le marché, occupera une place importante au sein de la recherche en émotion. En attendant, malgré toute l'excitation qu'ils suscitent, l'état actuel de ces systèmes ne les rend pas 
d'utilisation pratique et ils demeurent sensibles aux petits détails, comme par exemple le port de lunettes. II est difficile d'estimer le temps qu'il faudra à ces systèmes pour arriver à maturité; un délai d'une décennie est peut-être déjà trop optimiste. La raison derrière ce délai n'est pas liée à un ralentissement du développement technologique, mais bien aux problèmes conceptuels auxquels ces systèmes font face aujourd'hui. La solution passe par un accroissement de la recherche fondamentale sur les expressions faciales liées aux émotions!

On est en droit de se demander s'il est nécessaire de s'encombrer de toute cette technologie et de ces méthodes complexes pour étudier les expressions faciales. À notre avis, la réponse est un «oui» catégorique. Lorsqu'on veut véritablement concentrer ses recherches sur les expressions faciales, le fait d'asseoir ces recherches sur des jugements subjectifs provenant de groupes de juges naïfs, ou même de juges expérimentés comme les cliniciens, sans avoir recours à un système bien défini et validé, peut être considéré comme de la «mauvaise science». Les gens ne sont pas des machines à décoder les expressions faciales, même s'ils sont capables d'en extraire certaines informations valides. Ils peuvent d'ailleurs être influencés dans leurs jugements par une multitude de facteurs (Kappas, 1991). De même, il n'y a aucune raison de se limiter à la différenciation entre affect positif et négatif; le visage représente une source si riche d'informations qu'il serait dommage de ne pas l'utiliser au maximum.

On a aussi utilisé les fluctuations de la voix pour indexer les changements d'états émotionnels (e.g., Kappas, Hess, \& Scherer, 1991; Pittam \& Scherer, 1993; Johnstone \& Scherer, 2000; voir aussi Kappas, sous presse-a; Kappas \& Descôteaux, sous presse). Cependant, le recours aux paramètres acoustiques pour mesurer les affects continue d'être marqué par un certain nombre de problèmes pratiques et conceptuels qui découragent les chercheurs à utiliser cette méthode. Malgré l'existence de méthodes élaborées facilitant leur utilisation (i.e., Frey, Hirsbrunner, Pool, \& Daw, 1981), les changements posturaux et les gestes ne figurent pas plus souvent au sein des protocoles de recherche en émotion. Les raisons probables derrière cet anonymat consistent en ce que l'utilisation de la voix, de la posture et de la gestuelle nécessite l'apprentissage de connaissances sophistiquées, le recours à des équipements coûteux et la présence de ressources importantes permettant de mesurer plusieurs paramètres non verbaux en même temps. En effet, l'interaction entre les différents canaux de 
communication non verbale demeure complexe, et des progrès importants pourraient être accomplis suite à l'étude simultanée de plusieurs canaux de communication (Hess, Kappas, \& Scherer, 1988).

II est à noter que le «Handbook of methods in Nonverbal Behavior Research» (Scherer \& Ekman, 1982) continue d'être le texte de référence dans ce domaine. Ceux qui sont intéressés par la mesure des comportements non verbaux au sein de leurs recherches y trouveront une foule d'informations utiles concernant la méthodologie, la technologie, l'analyse des données ainsi qu'une revue des problèmes conceptuels inhérents à ce domaine.

\subsection{Les méthodes psychophysiologiques}

Les mesures psychophysiologiques font partie de la tradition de recherche en psychologie de l'émotion (voir Cacioppo \& Tassinary, 1990; Pecchinenda, 200 I). En effet, puisque la plupart des théories accordent une place importante à l'activation du système nerveux autonome au sein du processus émotionnel, maints travaux ont utilisé ce genre de mesure. Elles ont cependant joué des fonctions différentes selon l'orientation théorique des chercheurs et les questions de recherche étudiées:

- Vérification générale de la manipulation d'induction (l'activation indique la présence d'affects)

- Mesure de l'intensité (le degré d'activation indique l'intensité de l'état affectif)

- Marqueur spécifique (indique la présence, et peut-être même l'intensité, d'un affect spécifique)

La fonction octroyée aux mesures psychophysiologiques par les chercheurs dépend évidemment de la position qu'ils prennent au sein du débat toujours actuel qui découle de l'attaque virulente de Cannon (1927) à l'endroit de la théorie de James-Lange ${ }^{(I)}$. En bout de ligne, cependant, des

(1) II importe de noter que le problème a été jusqu'à présent de démontrer l'existence de patrons d'activation physiologique qui sont associés de façon fiable à certaines émotions spécifiques. La plupart des chercheurs dans ce domaine n'accepteraient pas la proposition de Cannon ou de Schachter à l'effet que les données suggèrent l'existence d'une «activation générale» non spécifique. 
considérations pratiques s'ajoutent à cette position théorique et influencent profondément le choix des chercheurs quant à la nature et au nombre de variables utilisées. En effet, les mesures psychophysiologiques sont reconnues pour leur complexité et leur coût élevé. Pour justifier leur usage à grande échelle, il faudrait réaliser une revue en profondeur de leurs vertus et limites en regard de la recherche en émotion.

La bonne nouvelle, c'est que la mesure de l'activité psychophysiologique en périphérie a beaucoup évolué depuis le moment où Albert $A x$ commençait à publier sa revue(2), qui visait à l'époque à répandre les connaissances techniques requises par l'utilisation de ce genre de mesure. Plusieurs compagnies offrent maintenant des appareils qui sont robustes, faciles à installer et relativement abordables. Les mesures sont maintenant enregistrées directement à l'ordinateur au lieu du désuet polygraphe à papier, ce qui augmente la flexibilité dans le choix des analyses et diminue le nombre d'erreurs dues à la digitalisation des données à partir des enregistrements papier.

La mauvaise nouvelle, c'est que les notions concernant les mécanismes d'activation psychophysiologique sur lesquelles reposent la plupart des théories classiques et actuelles sont désuètes et périmées. Par exemple, la populaire mesure de la fréquence cardiaque constitue probablement un des pires indices de l'activation du système sympathique. En fait, les discordances qu'on observe entre les études quant aux patrons d'activation liés aux émotions peuvent non seulement être dues aux particularités des procédures utilisées, comme par exemple la méthode d'induction choisie, mais aussi aux variations interindividuelles quant à l'influence qu'exercent les systèmes sympathique et parasympathique sur la fréquence cardiaque (e.g., Berntson, Cacioppo, \& Quigley, 1991). Par ailleurs, Porges (1995) a récemment proposé un nouvelle théorie dite «polyvagale» qui permet d'éclairer certains phénomènes qui jusqu'à ce jour demeuraient inexplicables par les théories «unitaires». Les mesures qu'on considère aujourd'hui comme représentant le mieux l'influence des systèmes sympathique et parasym-pathique sont respectivement le PEP («pre-ejection period») et le RSA («respiratory sinus arrhythmia»). Or, l'obtention fiable de ces indices demeure

(2) La «newsletter» est éventuellement devenue le «Journal of Psychophysiology», actuellement le journal de pointe dans le domaine de la psychophysiologie. 
une affaire coûteuse et compliquée (voir Papillo \& Shapiro, 1990). Pourtant, insister sur l'utilisation de paramètres qu'on sait être de mauvais indicateurs des processus qu'on veut mesurer en revient à faire comme l'alcoolique de la vieille blague qui, ayant perdu ses clefs, les cherche sous le lampadaire, non parce qu'il les a perdues là, mais parce que c'est le seul endroit où il peut voir quelque chose!

En plus des problèmes conceptuels liés au choix des paramètres, il y a encore ceux associés aux contextes expérimentaux fréquemment utilisés (voir également ci-dessus). Plusieurs chercheurs en émotion portent un intérêt particulier aux mesures physiologiques parce qu'ils les considèrent non réactives, relativement non obstructives, et imperméables à l'influence des demandes sociales et situationnelles, par opposition aux autres mesures disponibles, comme les rapports subjectifs et les expressions faciales. Or, des opinions à ce point optimistes ne prennent simplement pas en considération la réalité du contexte du laboratoire de psychophysiologie, où l'effet des consignes implicites de la part de l'expérimentateur, l'effet du contexte et les effets sociaux sont tout aussi présents (voir Fridlund \& Cacioppo, 1986). Pis encore, plusieurs chercheurs tendent à oublier l'origine fonctionnelle des réactions physiologiques périphériques. Pour fins d'illustration, considérons un plan expérimental visant à comparer les changements physiologiques induits par une randonnée de bicyclette en montagne à ceux provoqués par un trajet en terrain plat. Imaginons que le chercheur responsable de l'étude invite les participants à s'asseoir confortablement dans un fauteuil et leur demande de s'imaginer être en train de conduire une bicyclette. Une telle étude semble farfelue; après tout, les changements physiologiques découlent des actions et non de l'imagination des participants. Néanmoins, il est vrai que le simple fait d'imaginer conduire une bicyclette, jouer au tennis, ou même lire est suffisant pour provoquer des actions musculaires détectables par l'EMG. Toutefois, il est fort improbable que l'imagination puisse recréer les changements cardiovasculaires liés aux actions elles-mêmes. Comment pouvons-nous penser, alors, étudier la colère en assoyant confortablement nos participants dans un fauteuil? Combien de fois nous arrive-t-il de nous allonger et de relaxer lorsque nous avons peur? Cela peut nous arriver, si nous faisons par exemple partie d'un programme de désensibilisation systématique visant à contrer les effets de la peur. Se peut-il alors que nous favorisions le «découplage» des composantes de l'émotion lorsque nous faisons appel aux contextes expérimentaux conventionnels? 
Nous devons sérieusement repenser l'utilisation du mouvement et de l'action dans le laboratoire, puisque sous prétexte d'éliminer les artefacts dus aux gestes des participants, nous provoquons en eux des états affectifs et physiologiques qui sont bien loin des processus que nous désirons réellement étudier.

Le champ a néanmoins connu des développements intéressants au cours des dernières années, par exemple la construction de petites unités portables qui permettent d'enregistrer certains paramètres physiologiques alors que les participants se trouvent dans des situations émotionnelles véritables. La portée de cette innovation demeure toutefois limitée puisqu'il est difficile de trouver des situations qui sont réellement comparables et qu'un trop grand nombre de variables sont modifiées en même temps. Peut-être serait-il avantageux d'utiliser les unités portables dans l'environnement du laboratoire, combinant ainsi flexibilité de mouvement pour les participants et contrôle rigoureux des variables pour le chercheur.

La plus importante évolution récente demeure sans doute la création de nouveaux moyens pour mesurer l'activité du système nerveux central (SNC). Ces moyens comprennent des procédés électrophysiologiques comme l'électroencéphalographie (EEG) et les potentiels évoqués («event-related potentials; ERP»), ainsi que des méthodes d'imagerie neurale comme la tomodensitométrie par émission de positrons («Positron Emision Tomography; PET»), l'imagerie fonctionnelle par résonance magnétique («functional Magnetic Resonance Imaging; $\mathbb{f M R} \mid \gg$ ), et la magnétoencéphalographie («Magnetoencephalography; MEG»). Cependant, la plupart de ces techniques créent des situations fortement artificielles qui rendent l'étude des états émotionnels difficile.

Bien que les données provenant des méthodes d'imagerie neurale soient impressionnantes, il est clair qu'elles ne rendent pas obsolète la contribution des mesures de l'activation physiologique en périphérie, du comportement expressif et du rapport subjectif. En effet, le développement d'une vue d'ensemble du processus émotionnel requiert certainement une compréhension multifactorielle au sein de laquelle le rôle joué par le SNC occupe une place cruciale, mais là n'est pas toute la question. Partant, il importe de tempérer l'enthousiasme exagéré manifesté actuellement à l'égard des mesures de l'activation centrale tout en encourageant ceux qui hésitent encore à les ajouter à leur arsenal de mesures. II est également important de réaliser que les limites des mesures de l'activité centrale ne sont pas que technologiques - on les repoussera sûrement au cours des 
prochaines années - mais aussi conceptuelles. Même si nous pouvions lire les pensées, plusieurs processus essentiels continueraient à nous échapper. Les émotions sont assurément produites au sein du cerveau, et celui-ci contribue certainement à la gestion de l'ensemble des réponses, mais il n'en demeure pas moins que l'impact de plusieurs facteurs, allant de la phylogenèse aux contextes social et culturel, reste difficile à comprendre à partir des seuls mécanismes du SNC.

\subsection{Les rapports subjectifs et la question des composantes divergentes}

II est logique d'explorer les tenants et aboutissants des expressions faciales et des mesures physiologiques dans une discussion comme celle-ci, qui porte principalement sur la technique, mais qu'en est-il de la mesure de l'expérience subjective? Alors que les inventaires de personnalité suivent un développement lent et laborieux, les échelles utilisées pour les rapports subjectifs des émotions sont construite, à peu d'exceptions près, de façon ad-hoc (i.e., l'échelle DES de Izard - voir Philippot, 1993; voir aussi Schorr, 200 I). II existe bien quelques questionnaires et listes dont le but est d'évaluer les humeurs, mais le problème associé à la mesure des états affectifs ne relève pas de la technique - il est essentiellement conceptuel. Dit carrément, il consiste en ce que nous ne savons pas réellement à quel point nous avons accès à nos propres sentiments subjectifs, et de quelle façon. Cette question primordiale requiert un traitement d'urgence qui tarde pourtant à venir (voir Buck, 1993; Dennett, 1991).

La question du genre de relations qui existent entre les composantes de l'émotion demande également à être débattue. Que devons-nous conclure lorsque le comportement expressif suggère la présence d'un premier état affectif, les réponses physiologiques et centrales indiquent la présence d'un second état, différent du premier, et le rapport subjectif l'existence d'un troisième état distinct des deux autres? Cette question a bien été soulevée par quelques auteurs (e.g., Lang, 1979), mais demeure foncièrement ignorée dans le quotidien des chercheurs. Le problème semble partiellement résulter de la scission qui existe entre les différentes approches, i.e. biologique, sociale et fonctionnelle. Certains chercheurs rejettent l'existence de l'influence du contexte social sous prétexte qu'elle appartient à la tutelle des «sciences molles», alors que d'autres veulent jeter le bébé 
avec l'eau du bain en abolissant le terme «émotion», une solution aussi vielle et commune que non constructive. II est facile de réciter pour la forme le sermon bien connu voulant que la psychologie de l'émotion inclut des éléments biologiques, sociaux, cognitifs, développementaux, cliniques, éthologiques, anthropologiques, et philosophiques ${ }^{(3)}$ - mais il est beaucoup plus difficile d'honorer en profondeur la complexité du phénomène émotionnel.

L'émergence de la neuroscience sociale (e.g., Cacioppo \& Berntson, 1992; Cacioppo, Berntson, \& Crites, 1996) a marqué un net progrès au sein de ce débat. Selon les auteurs cités entre parenthèses, les comportements peuvent être étudiés à différents niveaux, allant du plan cellulaire, organismique, systémique ou corporel au plan des interactions qui se produisent dans les contextes culturels et sociaux. Ils ont formulé trois principes généraux selon lesquels les interactions entre ces différents niveaux d'organisation sont déterminées:

( I) le principe du déterminisme multiple - l'élément cible d'un niveau d'organisation (i.e., réponse neuroeffectrice, prédisposition envers certaines réponses évaluatives) peut compter des antécédents en provenance soit du même niveau, soit de niveaux différents; (2) le principe du déterminisme non additif - les propriétés de l'ensemble ne peuvent toujours être prédites à partir des propriétés des parties, à moins que les propriétés de l'ensemble n'aient été clairement étudiées et documentées en fonction des différents niveaux d'organisation; et (3) le principe du déterminisme réciproque - les processus neurologiques centraux et comportementaux peuvent être déterminés par l'influence mutuelle qu'exercent entre eux les facteurs microscopique (i.e., biologique) et macroscopique (i.e., social; Cacioppo et al., 1996, p. 73).

II est clair que la recherche en émotion va fortement bénéficier de l'approche de la neuroscience sociale. En effet, elle permet d'interpréter le rapport subjectif non comme un indicateur externe du sentiment intérieur d'un individu, mais comme l'indication d'une réponse émotionnelle à un niveau d'organisation donné, qui demeure en interaction constante avec les autres réactions émotionnelles. Kappas mentionnait justement dans le cadre de sa propre théorie de l'émotion («Dynamic Appraisal Theory of

(3) Nos excuses les plus sincères aux membres des disciplines qui n'ont pas été mentionnées. 
Emotions»; Kappas, 1996; Kappas, 200 I) que le rapport subjectif résulte de plusieurs déterminants, comme le résultat des évaluations cognitives, les influences sociales, et l'influence résiduelle des humeurs précédentes. Néanmoins, l'approche de la neuroscience sociale demeure évidemment indépendante des positions théoriques particulières (par exemple, le rôle de l'évaluation cognitive dans le déclenchement de l'émotion ou l'existence «d'émotions fondamentales» véritables) et constitue un paradigme général pour la recherche en émotion (Kappas, sous presse-b).

\subsection{Perspectives d'avenir}

Les cent dernières années ont été marquées par une foule de développements stimulants au plan de la recherche en émotion, tant à l'intérieur qu'à l'extérieur du laboratoire. Durant cette période, les chercheurs se sont graduellement regroupés autour d'un consensus quant à ce que sont les émotions et quels en sont les mécanismes de déclenchement. Cependant, le processus de recherche lui-même continue de dépasser en importance n'importe quelle méthode d'induction ou mesure particulière. En effet, rigueur et précision sont de mise malgré la complexité des questions auxquelles tente de répondre la recherche en émotion. La découverte du fonctionnement des émotions ne passe pas par l'adoption de telle ou telle méthode ou mesure. Les programmes de recherche porteront fruit en autant qu'ils aient recours à une variété de ces méthodes et mesures. Les principes de la neuroscience sociale devront être respectés, supportant ainsi l'étude de l'émotion à partir de niveaux d'organisation multiples. Chaque étude ne représente qu'un seul élément d'une histoire compliquée, en constante évolution, et seule une approche multidisciplinaire permettra de répondre aux questions comme: quel est le lien entre cognition et émotion?

\section{RÉFÉRENCES}

Ax, A. F. (1953). The physiological differentiation between fear and anger in humans. Psychosomatic Medicine, 15, 433-442.

Berntson, G. G., Cacioppo, J.T., \& Quigley, K. S. (199I). Autonomic determinism: The modes of autonomic control, the doctrine of autonomic space, and the laws of autonomic restraint. Psychological Review, 98, 459-487. 
Bonnet, M., Bradley, M. M., Lang, P. J., \& Requin, J. (1995). Modulation of spinal reflexes: Arousal, pleasure, action. Psychophysiology, 32, 367-372.

Buchwald, A.M., Strack, S., \& Coyne, J.C. (1981). Demand characteristics and the Velten mood induction procedure. Journal of Consulting and Clinical Psychology, 49, 478-479.

Buck, R. (1993). What is this thing called subjective experience? Reflections on the neuropsychology of qualia. Neuropsychology, 7, 490-499.

Cacioppo, J.T., \& Berntson, G. G. (1992). Social psychological contributions to the decade of the brain: The doctrine of multilevel analysis. American Psychologist, 47, 1019-1028.

Cacioppo, J. T., Berntson, G. G., \& Crites, S. L. J. (1996). Social neuroscience: Principles of psychophysiological arousal and response. In E.T. Higgins \& A. W. Kruglanski (Eds.), Social psychology: Handbook of basic principles (pp. 72-101). New York, NY: Guilford.

Cacioppo, J. T., Petty, R. E., Losch, M. E., \& Kim, H. S. (1986). Electromyographic activity over facial muscle regions can discriminate the valence and intensity of affective reactions. Journal of Personality and Social Psychology, 50, 260-268.

Cacioppo, J. T., \& Tassinary, L. G. (Eds.). (1990). Principles of psychophysiology. Cambridge: Cambridge University Press.

Cannon, W. B. (1927). The James-Lange theory of emotions: A critical examination and an alternative theory. American Journal of Psychology, 39, 106-124.

Center for the Study of Emotion and Attention (Csea). (1994). International Affective Picture System (IAPS). Gainesville, FL: University of Florida.

Cohn, J. F., Zlochower, A., Lien, J., \& Kanade, T. (1999). Automated face analysis by feature point tracking has high concurrent validity with manual FACS coding. Psychophysiology, 36, 35-43. Darwin, C. (1998). The expression of the emotions in man and animals: Definitive edition (Edited by Paul Ekman). Oxford: Oxford University Press (original published in 1872).

Darwin, C. (1998). The expression of the emotions in man and animals: Definitive edition (Edited by Paul Ekman). Oxford: Oxford University Press.

Darwin, C. (1872/1965). The expression of the emotions in man and animals. Chicago: The University of Chicago Press. (Originally published, 1872).

Dennett, D. C. (1991). Consciousness explained. Boston: Little, Brown and Company.

Duchenne, G. B. (1862/1990). The mechanism of human facial expression. (R.A. Cuthbertson, Ed. and Trans.). Cambridge: Cambridge University Press.

Ekman, P. (1984). Expression and the nature of emotion. In K. R. Scherer \& P. Ekman (Eds.), Approaches to emotion (pp. 319-343). Hillsdale: Erlbaum.

Ekman, P., \& Friesen, W.V. (1969). The repertoire of nonverbal behavior: Categories, origins, usage, and coding. Semiotica, 1, 49-98.

Ekman, P., \& Friesen, W. V. (1978). The Facial Action Coding System: A technique for the measurement of facial movement. Palo Alto, CA: Consulting Psychologists Press.

Frey, S., Hirsbrunner, H.-P., Pool, J., \& Daw, W. (198I). Das Berner System zur Untersuchung nonverbaler Interaktion: I. Die Erhebung des Rohdatenprotokolls. In P. Winkler (Ed.), Methoden der Analyse von Face-to-Face Situationen (pp. 203-236). Stuttgart: J.B. Metzlerische Verlagsbuchhandlung.

Fridlund, A. J. (1991). The sociality of solitary smiling: Potentiation by an implicit audience. Journal of Personality and Social Psychology, 60, 229-240.

Fridlund, A. J., \& Cacioppo, J.T. (1986). Guidelines for human electromyographic research. Psychophysiology, 23, 567-589 
Gerrads-Hesse, A., Spies, K., \& Hesse, F.W. (1994). Experimental inductions of emotional states and their effectiveness: A review. British Journal of Psychology, 85, 55-78.

Gross, J. J., \& Levenson, R.W. (1995). Emotion elicitation using films. Cognition \& Emotion, 9, 87-108.

Hamm, A. O., \& Vaitl, D. (1989). Multidimensionale Analyse affektiver visueller Stimuli: Eine transkulturelle Untersuchung / Multidimensional analysis of affective visual stimuli: A cross-cultural study. Psychologische Beiträge, 31, 125-143.

Hess, U., Banse, R., \& Kappas, A. (1995). The intensity of facial expression is determined by underlying affective state and social situation. Journal of Personality and Social Psychology, $69,280-288$.

Hess, U., Kappas, A., McHugo, G. J., Kleck, R. E., \& Lanzetta, J. T. (1989). An analysis of the encoding and decoding of spontaneous and posed smiles: The use of facial electromyography. Journal of Nonverbal Behavior, 13, 121-137.

Hess, U., Kappas, A., McHugo, G. J., Lanzetta, J. T., \& Kleck, R. E. (1992). The facilitative effect of facial expression on the self-generation of emotion. International Journal of Psychophysiology, 12, 25I-265.

Hess, U., Kappas, A., \& Scherer, K. R. (1988). Multichannel communication of emotion: Synthetic signal production. In K. R. Scherer (Ed.), Facets of emotion: Recent research (pp. |6|-| 82). Hillsdale, NJ: Lawrence Erlbaum Associates.

Hess, U., \& Kleck, R. E. (1990). Differentiating emotion elicited and deliberate emotional facial expressions. European Journal of Social Psychology, 20, 369-385.

Izard, C. E. (1979). The maximally discriminative facial movement coding system (MAX): Instructional Resources Center, University of Delaware.

Johnstone, T., \& Scherer, K. R. (2000). Vocal communication of emotion. In M. Lewis \& J. M. Haviland-Jones (Eds.), Handbook of emotions (2nd ed.) (pp. 220-235). New York, N.Y.: Guilford Press.

Kaiser, S., \& Wehrle, T. (1992). Automated coding of facial behavior in human-computer interactions with FACS. Journal of Nonverbal Behavior, 16, 67-84.

Kaiser, S., \& Wehrle, T. (200I). Facial expressions as indicators of appraisal processes. In K. R. Scherer, A. Schorr, \& T. Johnstone (Eds.), Appraisal processes in emotion (pp. 285-300). New York, N.Y.: Oxford University Press.

Kappas, A. (1989). Control of emotion. Unpublished doctoral thesis, Dartmouth College, $\mathrm{NH}$. Kappas, A. (|99|). The illusion of the neutral observer: On the communication of emotion. Cahiers de Linguistique Française de I'Université de Genève, 12, 153-168.

Kappas, A. (1992). CODER2: Computer assisted assessment of behavior. Paper presented at the Fifth European Conference on Facial Measurement, Bologna, Italy.

Kappas, A. (1996). The sociality of appraisals: Impact of social situations on the evaluation of emotion antecedent events and physiological and expressive reactions, IXth conference of the International Society for Research on Emotions (pp. I|6-120). Toronto, ON.

Kappas, A. (200I). A metaphor is a metaphor is a metaphor: Exorcising the homunculus from appraisal theory. In K. R. Scherer, A. Schorr, \& J. T. (Eds.), Appraisal processes in emotion: Theory, methods, research (pp. 157-172). New York, NY: Oxford University Press.

Kappas, A. (sous presse-a). What facial expressions can and cannot tell us about emotions. In M. Katsikitis (Ed.), The human face: Measurement and meaning . Dordrecht: Kluwer.

Kappas, A. (sous presse-b). The science of emotion as a multidisciplinary research paradigm. Behaviour Research. 
Kappas, A., \& Descôteaux, J. (sous presse). Of butterflies and roaring thunder: Nonverbal communication in interaction and regulation of emotion. In P. Philippot, E. J. Coats, \& R. S. Feldman (Eds.), Nonverbal behavior in clinical settings. New York, NY: Oxford University Press.

Kappas, A., \& Hess, U. (1995). Nonverbal aspects of oral communication. In U. M. Quasthoff (Ed.), Aspects of oral communication (pp. 169-180). Berlin: DeGruyter.

Kappas, A., Hess, U., \& Banse, R. (1992). Skin conductance reactions to dynamic facial expressions revisited: Empathic responding or information processing? Psychophysiology, 29, 542 .

Kappas, A., Hess, U., \& Scherer, K. R. (199|). Voice and emotion. In R. S. Feldman \& B. Rimé (Eds.), Fundamentals of nonverbal behavior (pp. 200-238). Cambridge: Cambridge University Press.

Kappas, A., \& Pecchinenda, A. (1999). Don't wait for the monsters to get you: A video game task to manipulate appraisals in real time. Cognition and Emotion, 13, 119-124.

Kirouac, G. (1995). Les Émotions (2e édition). Sainte-Foy: Presses de l'Université du Québec.

Kleck, R. E., Vaughan, R.C., Cartwright-Smith, J., Vaughan, K.B., Colby, C.Z., \& Lanzetta, J.T. (1976). Effects of being observed on expressive, subjective, and physiological responses to painful stimuli. Journal of Personality and Social Psychology, 34, 1211-1218.

Landis, C. (1924a). Studies of emotional reactions: I. A preliminary study of facial expression. Journal of Experimental Psychology, 7, 325-341.

Landis, C. (1924b). Studies of emotional reactions: II. General behavior and facial expression. Journal of Comparative Psychology, 4, 447-509.

Landis, C., \& Gullette, R. (1925). Studies of emotional reactions: III. Systolic blood pressure and inspiration-expiration ratios. Journal of Comparative Psychology, 5, 221-253.

Lang, P. J. (1979). A bio-informational theory of emotional imagery. Psychophysiology, 16, 495-512 .

Lang, P. J., Greenwald, M.W., Bradley, M. M., \& Hamm, A. O. (1993). Looking at pictures: Affective, facial, visceral, and behavioral reactions. Psychophysiology, 30, 26I-273.

Larsen, R. J., \& Sinnett, L. M. (|99|). Meta-analysis of experimental manipulations: Some factors affecting the Velten Mood Induction Procedure. Personality and Social Psychology Bulletin, 17, 323-334.

Leventhal, H., \& Scherer, K. (1987). The relationship of emotion to cognition: A functional approach to a semantic controversy. Cognition and Emotion, 1, 3-28.

Martin, M. (1990). On the induction of mood. Clinical Psychology Review, 10, 669-697.

154 McHugo, G. J., Smith, C. A., \& Lanzetta, J.T. (1982). The structure of self-reports of emotional responses to film segments. Motivation and Emotion, 6, 365-385.

Noldus, L. P., Trienes, R. J. H., Hendriksen, A. H. M., Jansen, H., \& Jansen, R. G. (2000). The Observer Video-Pro: New software for the collection, management, and presentation of time-structured data from videotapes and digital media files. Behavior Research Methods, Instruments and Computers, 32, 197-206.

Noldus, L. P., Van de Loo, E. L., \& Timmers, P. H. (1989). Computers in behavioural research. Nature, 341, 767-768.

Öhman, A. (1993). Stimulus prepotency and fear learning: Data and theory. In N. Birbaumer \& A. Öhman (Eds.), The organization of emotion: Cognitive, clinical, and psychophysiological perspectives. (pp. 218-239). Toronto: Hogrefe. 
Oster, H., Hegley, D., \& Nagel, L. (1992). Adult judgments and fine-grained analysis of infant facial expressions:Testing the validity of a priori coding formulas. Developmental Psychology, 28, $1115-1131$.

Papillo, J. F., \& Shapiro, D. (1990). The cardiovascular system. In J.T. Cacioppo \& L. G. Tassinary (Eds.), Principles of psychophysiology (pp. 456-5 12). Cambridge: Cambridge University Press. Pecchinenda, A. (200I). The psychophysiology of appraisals. In K. R. Scherer, A. Schorr, \& T. Johnstone (Eds.), Appraisal processes in emotion: Theory, methods, research (pp. 30I-3।5). New York, N.Y:: Oxford University Press.

Philippot, P. (1993). Inducing and assessing differentiated emotion-feeling states in the laboratory. Cognition and Emotion, 7, 171-193.

Pittam, J., \& Scherer, K. R. (1993). Vocal expression and communication of emotion. In M. Lewis \& J. M. Haviland (Eds.), Handbook of emotions (pp. 185-197). New York: Guilford Press.

Porges, S. W. (1995). Orienting in a defensive world: Mammalian modifications of our evolutionary heritage: A polyvagal theory. Psychophysiology, 32, 301-318.

Rimé, B., Philippot, P., \& Cisamolo, D. (1990). Social schemata of peripheral changes in emotion. Journal of Personality and Social Psychology, 59, 38-49.

Rothbaum, B. O., Hodges, L., \& Kooper, R. (1997). Virtual reality exposure therapy. Journal of Psychotherapy Practice and Research, 6, 219-226.

Rowe, P. M. (1995, Mars). Is virtual reality technology only virtually here? APS Observer, 8, pp. 17, 37-38.

Sayette, M. A., Cohn, J. F., Wertz, J. M., Perrott, M. A., \& Parrott, D. J. (200I). A psychometric evaluation of the Facial Action Coding System for assessing spontaneous expression. Journal of Nonverbal Behavior, 25, 167-185.

Schachter, S., \& Singer, J. E. (1962). Cognitive, social, and physiological determinants of emotional state. Psychological Review, 69, 379-399.

Scherer, K. R. (1986). Vocal affect expression: A review and a model for future research. Psychological Bulletin, 99, 143-165.

Scherer, K. R. (1993). Neuroscience projections to current debates in emotion psychology. Cognition and Emotion, 7, |-4|.

Scherer, K. R. (200I). Appraisal considered as a process of multilevel sequential checking. In K. R. Scherer, A. Schorr, \& T. Johnstone (Eds.), Appraisal processes in emotion: Theory, methods, research (pp. 92-120). New York, NY: Oxford University Press.

Scherer, K. R., \& Ekman, P. (Eds.). (1982). Handbook of methods in nonverbal behavior research. Cambridge: Cambridge University Press.

Scherer, K. R., Schorr, A., \& Johnstone,T. (Eds.). (200 I). Appraisal processes in emotion: Theory, Methods, Research. New York, NY:: Oxford University Press.

Schorr, A. (200I). Subjective measurement in appraisal research: Present state and future perspectives. In K. R. Scherer, A. Schorr, \& T. Johnstone (Eds.), Appraisal processes in emotion: Theory, methods, research (pp. 331-349). New York, N.Y.: Oxford University Press.

Sinnett, L. M. (1995). Sex differences in reactivity to emotion manipulations: A meta-analysis, Nags Head Conference on Emotion and Motivation. Highland Beach, FLA.

Tassinary, L. G., Cacioppo, J.T., \& Geen, T. R. (1989). A psychometric study of surface electrode placements for facial electromyographic recording: I. The brow and cheek muscle regions. Psychophysiology, 26, I-16.

Tomkins, S. S. (1962). Affect, imagery, conciousness: The positive affects. New York: Springer.

Tomkins, S. S. (1963). Affect, imagery, conciousness: The negative affects. New York: Springer. 
Velten, E. (1968). A laboratory task for the induction of mood states. Behavior Research and Therapy, 6, 473-482.

Vrana, S. (1993). The psychophysiology of disgust: Differentiating negative emotional contexts with facial EMG. Psychophysiology, 30, 279-286.

Westermann, R., Spies, K., Stahl, G., \& Hesse, F.W. (1996). Relative effectiveness and validity of mood induction procedures: A meta-analysis. European Journal of Social Psychology, 26 , 557-580.

Yamada, H., Chiba, H., Tsuda, K., Maiya, K., \& Harashima, H. (1992). A facial image processing system for psychological studies. Paper presented at the IEEE International Workshop on Robot and Human Communication, Tokyo, 1-3 September 1992. 
Pio E. Ricci Bitti

Département de Psychologie

Université des Études de Bologne

\section{LA RÉGULATION DES COMPORTEMENTS EXPRESSIFS ÉMOTIONNELS}

\section{Les émotions et leur régulation}

L'émotion représente chez l'homme une des expériences les plus significatives; en effet, elle accompagne l'individu tout le long de son existence pour lui fournir un modèle d'adaptation quant aux interactions entre l'organisme et le milieu. Tous sont désormais amplement d'accord sur le fait que l'émotion doit être considérée comme une structure psychologique à l'intérieur de laquelle interviennent diverses composantes (Scherer, 1982): une composante cognitive ayant pour finalité l'évaluation de l'événement-stimulus qui provoque l'émotion; une composante d'activation physiologique déterminée principalement par l'intervention du système neurovégétatif; une composante constituée par l'expression non-verbale; une composante motivationnelle, relative aux intentions et à la tendance à agir/réagir; une composante subjective consistant dans le sentiment éprouvé par l'individu. Toutes les composantes sont interdépendantes, synchronisées, et concourent à déterminer l'expérience émotionnelle, qu'il faut donc considérer comme un processus dynamique plutôt que comme une condition stable ou permanente [lzard 1977; Plutchik 1980; Scherer 1982].

Les auteurs contemporains continuent à être en désaccord quant à la définition des fonctions et à la signification des émotions: s'il prévaut le point de vue tenant les émotions pour des mécanismes essentiellement adaptatifs et motivationnels, mis à la disposition de l'organisme pour affronter le milieu ambiant, l'idée que l'émotion représente une sorte d'interférence ou «interruption» par rapport au déroulement de séquences comportementales, jouit encore d'un certain crédit. 
On peut considérer l'émotion comme un élément de médiation entre les événements environnementaux en perpétuel changement et les réponses comportementales de l'homme. Scherer [1982] indique trois aspects fondamentaux de ce processus de médiation: l'évaluation cognitive relevant de la stimulation ou de l'événement environnemental par rapport aux besoins, aux plans et aux préférences de l'organisme; la préparation physiologique et psychologique à l'action nécessaire pour affronter d'une manière adéquate la stimulation; la communication-signalisation transmise par l'organisme face au milieu environnant de son propre état, ses propres intentions et réactions.

L'évolution du système des émotions est relativement importante chez les espèces sociales: leur expression non verbale constitue une communication-signalisation pour les autres organismes qui peuvent en conséquence prévoir les comportements de l'individu qui expérimente l'émotion et planifier, à leur tour, les actions de façon appropriée.

L'analyse de l'expression et de la communication des émotions chez l'homme conduit à certaines considérations sur le problème de la régulation et du contrôle de ces émotions ainsi que sur les effets qu'une telle régulation provoque chez l'individu (cf. Fig. I).

La régulation est une composante essentielle du processus émotionnel: cela signifie que les individus ne se limitent pas à «éprouver» des émotions, mais que de façons différentes ils «interviennent» sur leur expérience émotionnelle; en fait, durant toutes les phases de cette expérience, et face à toutes les composantes du processus peuvent éventuellement être adoptées des actions régulatrices, contribuant à en moduler certains aspects. Pour pouvoir réaliser une régulation du processus émotionnel, l'individu doit être capable d'effectuer un «suivi» constant du processus en question, en fonction duquel il adoptera des stratégies spécifiques de régulation( ${ }^{(1)}$.

Presque tous les aspects de la réponse émotionnelle, on le sait, peuvent être soumis à des actions régulatrices, en ce sens qu'ils peuvent être, par exemple, inhibés, atténués, intensifiés, ou en tous cas modifiés dans leur expression.

(1) Une telle façon de définir la régulation des émotions laisserait à penser que l'acte régulateur est volontaire ; au contraire, il convient de rappeler qu'il n'existe pas de séparation absolue entre des processus involontaires et des stratégies volontaires de régulation. 


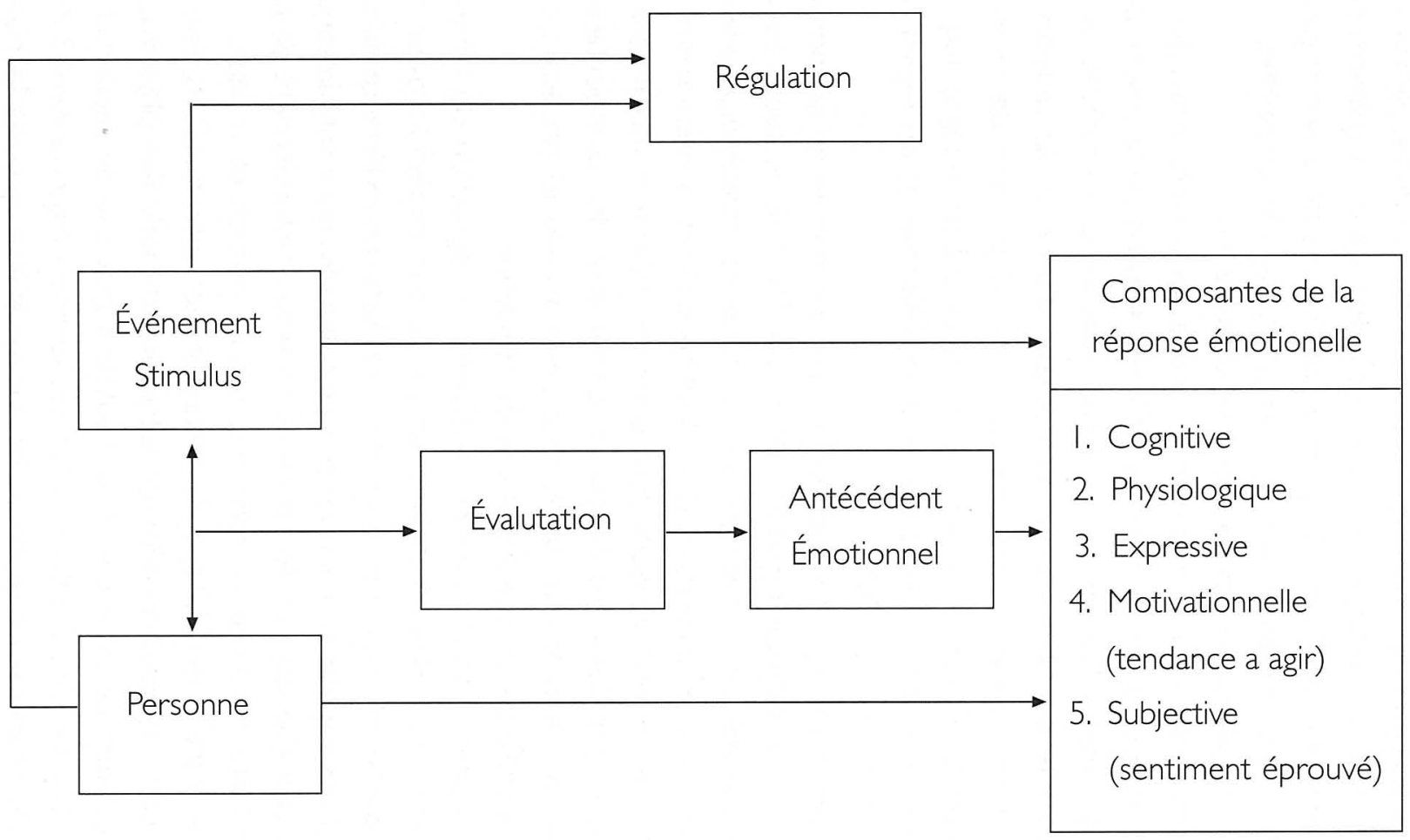

Figure I - Interdépendance entre les aspects de l'expérience émotionnelle 
Les objectifs des processus de régulation peuvent essentiellement être ramenés aux suivants: réduire ou augmenter les conséquences respectivement négatives ou positives des réponses comportementales induites par l'émotion et réduire le malaise ou accentuer le bien-être dérivant de l'expérimentation d'une certaine émotion. Dans le premier cas, on assiste à une régulation des résultats comportementaux de l'émotion, dans le deuxième, à une régulation intrapsychique des mécanismes d'évaluation.

La régulation peut donc concerner les processus cognitifs; nous pouvons citer à ce propos les mécanismes de défense dérivant de la psychanalyse, moyennant lesquels l'individu se «défend» contre une émotion dans la mesure où elle est désagréable ou porteur d'un état de tension non tolérable ou non approuvé socialement; il en est presque de même de certains aspects régulateurs des mécanismes de coping intrapsychique de Lazarus (1966) et les concepts d'évaluation défensive et de réévaluation constructive de Frijda (1986).

Les processus de régulation peuvent en outre intéresser les phénomènes d'activation physiologique, résultant de l'intervention du système nerveux autonome ou végétatif; un exemple très connu de ces stratégies régulatrices est représenté par les techniques du biofeed-back. D'autres mécanismes plus évidents et même plus analysés sont ceux de régulation mis en place à l'égard des expressions des émotions, c'est-à-dire des comportements observables qui découlent de la réponse émotionnelle. Ici, on fera surtout référence à cette partie des processus de régulation.

Par conséquent, nous pouvons considérer la régulation de l'émotion comme l'ensemble des stratégies adoptées par l'individu pour faire correspondre l'expérience intérieure et la manifestation extérieure de celleci aux situations sociales et aux normes socioculturelles qui y sont impliquées: il est donc clair que, dans un tel processus régulateur, interviennent de façon interdépendante des facteurs intérieurs, interpersonnels et sociaux.

$\mathrm{Si}$, d'une part, il est difficile de distinguer les interrelations entre le comportement expressif, l'expérience subjective et l'activation physiologique dans la régulation de l'émotion, il est toutefois plus aisé de reconnaitre le rôle des facteurs socioculturels; en effet, ces derniers interviennent à divers moments de l'expérience émotionelle: sur les stimuli qui, par le biais de l'apprentissage, sont en mesure de susciter des émotions précises, sur les règles qui en gouvernent la manifestation, sur la plupart des conséquences de l'activation émotionnelle [Ekman 1972; Scherer 1982]. 


\section{Exprimer les émotions et communiquer sur les émotions}

L'extériorisation des émotions, à savoir la modalité selon laquelle les émotions se manifestent extérieurement à travers la mimique faciale, les mouvements du corps, le langage et par le biais d'autres signaux plus ou moins repérables, a déjà fait depuis l'antiquité l'objet de nombreuses études. Cependant, ce n'est qu'avec Darwin (1872) que ces recherches se sont concentrées systématiquement autour de la fonction et de la signification de l'expression émotionnelle. Vues sous l'angle plus général du rapport homme-milieu, les réponses émotionnelles ont été tenues pour d'importants facteurs intervenant dans le but de réguler de manière adaptative la vie individuelle au sein d'un groupe social. L'expression et la reconnaissance d'états émotionnels déterminés permettent une plus grande capacité communicative entre l'individu et le groupe et garantissent une meilleure probabilité de survie à chacun d'entre eux. Pour appuyer cette thèse, on peut voir que seuls certains primates, en dehors de l'homme, possèdent un répertoire expressif au niveau de la mimique faciale suffisamment différenciée pour indiquer un certain nombre d'états émotionnels de base. Plus on monte dans l'échelle évolutive et examine des espèces vivant en communauté permanente, plus l'expressivité émotionnelle apparaît structurée et capable de communiquer des messages précis.

Ce n'est qu'à une époque relativement récente que les premières intuitions de Darwin ont été reprises par des éthologistes et par certains chercheurs tels que Ekman et Friesen (1969), lzard (197I), Tomkins (1980) et par d'autres ayant postulé l'existence de traits panculturels et universels dans l'expression faciale et la reconnaissance d'états émotionnels précis. D'autre part, on a vu (Ekman et Friesen, 1969) que, chez l'homme, contrairement aux primates, l'extériorisation des émotions peut, d'une certaine manière, être contrôlée intentionnellement. Ekman et Friesen (1969) ont parlé à ce propos des display rules, c'est-à-dire de règles d'exhibition influencées par les styles de comportement d'un groupe social déterminé. De plus, même si l'extériorisation des émotions représente un aspect capital de l'expérience émotionnelle chez l'homme, il faut dire que celle-ci interagit avec d'autres composantes de l'émotion, telles que, en l'occurrence, l'évaluation de la situation de stimulation, le vécu subjectif ou la disposition à agir. Ces autres composantes permettent de rattacher l'activité de l'expression non verbale des émotions à des aspects cognitifs et motivationnels particuliers. 
En d'autres termes, même si les réponses émotionnelles font partie du bagage biologique de l'être humain, elles sont suffisamment sophistiquées pour permettre à l'individu soit une évaluation de la situation extérieure en perpétuel changement, soit des vécus intérieurs. C'est-à-dire qu'elles permettent un rapport très souple avec le milieu.

Les éléments caractérisant l'extériorisation d'états émotionnels déterminés sont multiples et hétérogènes: parallèlement à une série de réponses physiologiques ou d'expressions faciales qui définissent de façon immédiate un type de réaction émotionnelle (comme la peur, la joie, la colère, la tristesse, la surprise ou le dégoût, pour rappeler celles que de nombreux spécialistes et auteurs mettent au rang des émotions fondamentales), il existe des messages (verbaux et non verbaux) qui servent à partager une expérience émotionnelle précise et d'en recueillir, entre les lignes, les nuances les plus infimes. Par conséquent, quand on parle de l'extériorisation des émotions, il peut être utile de faire une distinction entre expression et communication selon la nature du message extériorisé: à savoir, s'il est ou n'est pas intentionnel. En effet, ce n'est pas toujours que les aspects expressif et communicatif coïncident. Buck (1984) a proposé une subdivision entre la communication spontanée, propre à un système biologiquement partagé, se référant à un code d'origine génétique (pas appris) et la communication symbolique, propre à un système socialement partagé se rapportant à un code d'origine culturelle (appris). Pour Buck (1984) il existe donc un double code interprétatif qui délimite l'extériorisation des émotions et qui, de manière plus générale, représente sur un plan écologique le rapport entre l'homme et le milieu. L'aspect adaptatif de la communication spontanée se manifeste surtout dans le caractère non propositionnel de ce type de communication: aussi bien au niveau de la codification que de la décodification, les messages non

162 propositionnels donnent naissance à un rapport homme-milieu qui se précise dans un type de connaissance immédiate et dans une communication universellement partagée, ni volontaire, ni symbolique et ni même falsifiable. En revanche, la communication symbolique est caractérisée par un type de connaissance acquise par l'intermédiaire de messages propositionnels: elle est socialement partagée et fondamentalement intentionnelle et elle peut être falsifiable.

Reprenant ce qui a été énoncé plus haut au sujet du rapport entre expression et communication des émotions, on peut dire qu'à côté d'une 
fonction proprement adaptative (communication spontanée - expression des émotions) de l'extériorisation des émotions, il existe une fonction plus spécifiquement communicative, d'ordre symbolique, qui permet, si l'on se sert surtout d'instruments linguistiques, de communiquer sur les émotions, c'est-à-dire de partager un vécu émotionnel commun, en le réévoquant et le revivant ensemble. Tout ceci revêt une grande importance dans la détermination de ce qui touche aux effets sur l'individu mais aussi aux fonctions sociales ayant pour tâche de manifester extérieurement ses propres émotions.

\section{Styles de régulation et extériorisation émotionnelle}

Pour comprendre les effets des diverses modalités d'extériorisation des émotions, de nombreux chercheurs ont examiné le rapport entre les expressions faciales, plus facilement contrôlables, et les réponses physiologiques, involontaires de surcroît, dans le but de réussir à éclaircir quel est le poids qu'assument, au niveau de l'expression des émotions, les caractéristiques individuelles d'une part et d'autre part des situations sociales précises. Ces recherches se sont orientées en direction de deux axes théoriques. Le premier de ces modèles que l'on peut appeler celui de l'arousal met positivement en corrélation l'expression faciale avec l'activation physiologique (Lanzetta et al., 1976 ; Lanzetta et Kleck, 1970 ; Laird, 1974): l'expression émotionnelle proviendrait d'éléments variés qui agissent entre eux en synergie et se renforcent avec une série de feed-backs. Si l'on se réfère directement aux travaux de Darwin (1872), qui avait soutenu que l'expression émotionnelle intensifie l'expérience émotionnelle, de façon à ce que plus une émotion est exprimée, plus la sensation du sujet qui l'exprime est intense, ce modèle suit une hypothèse précise, connue sous le nom d' «hypothèse du feed-back facial» selon laquelle on suppose qu'un sujet exprimant librement et ouvertement ses propres émotions devrait avoir une réaction plus forte qui se traduirait aussi chez d'autres indicateurs émotionnels. En revanche, la deuxième théorie, qui a pris naissance à la suite des travaux de Jones (1935), a trouvé deux catégories de sujets - les intériorisateurs et les extériorisateurs - qui se différencient entre eux dans la mesure où les premiers présentent une expressivité faciale et mimique réduite ainsi qu'une haute réaction physiologique (électrodermale) tandis 
que les seconds présentent une forte expressivité extérieure et une faible activation physiologique. Ce modèle se rapproche du soi-disant modèle «cathartique», adopté tout du moins en partie par les théoriciens de la psychanalyse: dans ce cas, l'expression émotionnelle réduirait la tension intérieure. La corrélation inverse entre l'expressivité faciale et les réponses physiologiques constituerait une indication dans ce sens.

Si l'on se penche sur l'examen comparé des recherches ayant trait aux deux lignes théoriques mentionnées, il en émerge, d'une part, une nette confirmation de la discordance existant chez de nombreux sujets entre les indicateurs expressifs faciaux et les réponses physiologiques (Buck et al., 1972 ; Buck et al., 1974 ; Notarius et Levenson, 1979 ; Notarius et al., 1982; Field et Walden, 1982) et, d'autre part, dans les cas de forte anxiété, une activation générale des deux (Asendorpf et Scherer, 1983). Par contre, les confirmations spécifiques à l'hypothèse du feed-back facial (Buck, 1984) se sont avérées plus limitées. Même si les travaux de Field et Walden (1982) ont mis en évidence qu'il existait, dès la prime enfance, des styles expressifs différents du modèle intériorisation/extériorisation, il convient encore d'ajouter que les modes d'intériorisation et d'extériorisation peuvent être également appris en vertu de rôles sociaux déterminés, si bien qu'un individu peut agir selon une modalité intériorisatrice ou extériorisatrice en fonction de la situation où il se trouve.

On peut expliquer les discordances apparues au cours des travaux sur les modalités extériorisatrices et intériorisatrices, à propos des divers indicateurs des émotions par le fait que la dimension émotionnelle chez l'homme est organisée, on le sait, selon différents niveaux hiérarchiques, des plus archaïques aux plus évolutifs, et que chacun d'entre eux présente des systèmes divers de contrôle et de suivi des informations (Scherer, 1984). Si l'on considère l'hypothèse de Darwin relative au rapport entre expressivité et expérience émotionnelles, il faut analyser comment le passage de la sphère de l'expressivité à celle de l'expérience n'est pas automatique, mais s'exerce par l'intermédiaire d'une série d'éléments d'évaluation.

De nombreuses recherches sur les différents styles de régulation de l'activité émotionnelle effectuée par différents individus d'une part et d'autre part sur les effets que ces styles produisent sur ces mêmes individus à court et long terme ont mis en relief des typologies aux contours définis: Jones (1935), déjà cité, distingue deux catégories de sujet - les intériorisateurs et les extériorisateurs - qui se différencient entre eux dans la mesure où 
les premiers présentent une faible expressivité non verbale et une haute réaction physiologique, tandis que les deuxièmes présentent une forte expressivité extérieure et une faible activation physiologique; dans cette même ligne, Byrne (1964) a divisé en «repressors» et «sensitizers» les sujets extériorisant leurs émotions de différentes façons; la distinction traditionnelle proposée par Eysenk (1957) entre introversion et extroversion ainsi que la notion de «locus of control» extérieure et intérieure (Nowicki et Duke, 1974) sans oublier la «théorie de la polarité» (Tomkins, 1982) reproposent, elles aussi, même si elles le font selon des modalités diverses, une bipartition «intérieure-extérieure» à appliquer aux signaux émotionnels en fonction de leur «visibilité». || est important de noter comment tous ces modèles se réfèrent essentiellement à l'expression plutôt qu'à la communication des émotions. Parallèlement aux différences touchant les styles expressifs individuels, certaines typologies trouvent des réponses psychopathologiques, indices d'un équilibre altéré par rapport au milieu. II est intéressant de noter que c'est justement la polarité expressive (émotivité ; locus of control extérieur) qui est principalement impliquée dans les réponses de type pathologique. II semblerait donc que plus les émotions s'extériorisent, plus le bien-être psychophysiologique de l'individu fait défaut. En réalité tout ceci est vrai seulement si la prédominance du pôle expressif renvoie à une formation altérée du Soi, si bien qu'un individu n'est pas capable de contrôler ses propres vécus émotionnels ni de garder pour soi la «douleur mentale» qui en dérive (Bion, 1962).

D'autres recherches ont montré comment l'inhibition de la communication émotionnelle avait de profondes répercussions sur l'état de santé, surtout en présence de faits traumatisants. Pennebaker et O'Heeron (1984) ont fait voir comment les conjoints de personnes décédées à la suite d'un suicide ou d'un accident présentaient plus fréquemment des troubles de santé s'ils n'avaient pas pu ou pas voulu communiquer aux autres leurs émotions pendant le deuil. Cependant, contrairement aux autres recherches citées plus haut, celles-ci ont trait de façon prépondérante aux aspects relatifs à la communication symbolique des émotions: l'inhibition expressive coïncide dans ces cas-là avec l'impossibilité de communiquer à travers le langage (ou d'autres instruments symboliques non verbaux) l'expérience émotionnelle et, ce faisant, de l'élaborer. Rimé (1989) a signalé que la possibilité de revivre et évoquer à nouveau ses propres émotions protégeait contre les «effets à long terme» des événements traumatisants, alors qu'«à court terme» 
l'inhibition expressive pouvait atténuer la douleur indubitablement réveillée par le souvenir. Toutefois, même ici, il est bon de rester sur ses gardes et ne pas confondre les niveaux expressif et communicatif. Quand Rimé (1989) parle d' «effets à long terme», il entend surtout par là le dernier niveau: l'aménagement des souvenirs dans l'espace et le temps, appelé «déroulement» par l'auteur et correspondant à la condition préalable requise pour la reviviscence des émotions, consiste en pratique dans la création d'un univers symbolique. En revanche, pour ce qui est des «effets à court terme», ce sont surtout les aspects expressifs qui sont pris en considération (cf. Fig. 2).

La distinction entre les aspects expressifs et communicatifs au niveau de l'extériorisation des émotions est d'une importance cruciale dans le domaine clinique: l'incapacité à verbaliser ses émotions et dont de nombreux patients font preuve (trouble auquel Sifneos a donné le nom d'alexithymia, 1973, et que Marty et De M'Uzan,1963, ont défini «pensée opératoire») et qui, en grande partie, est étroitement liée à l'origine des troubles psychosomatiques, est tenue pour une véritable impossibilité d'élaborer des émotions et de construire son propre monde intérieur plutôt que pour une incapacité de type expressif (cf. Ricci Bitti e Gremigni, 1995; Ricci Bitti, Gremigni, Bertolotti, Zotti, 1995).

\section{Communication linguistique et élaboration symbolique de l'expérience émotionnelle.}

Pour pouvoir définir (et donc communiquer sur) son état émotionnel, il s'avère nécessaire d'avoir une certaine capacité d'auto-observation, une distance sûre entre le Soi et l'objet observé de façon à pouvoir considérer l'émotion vécue, même si on en reconnaît le caractère d'expérience

166 personnelle, comme autre par rapport à soi. À ce sujet, tant les travaux à caractère psychanalytique se référant au modèle des relations objectales, en particulier ceux de Winnicott (197I), que les contributions de spécialistes ayant décrit l'expérience émotionnelle en termes situationnels, surtout Frijda (1986) et Rimé (1989), illustrent très bien comment la possibilité de dénommer les émotions se rattache à la naissance et au développement des rapports interpersonnels. Dénommer les émotions favorise et soutient le lien avec l'autre: la capacité de s'observer et d'évaluer ses expériences émotionnelles fait partie intégrante du rapport interactif et permet d'éviter 


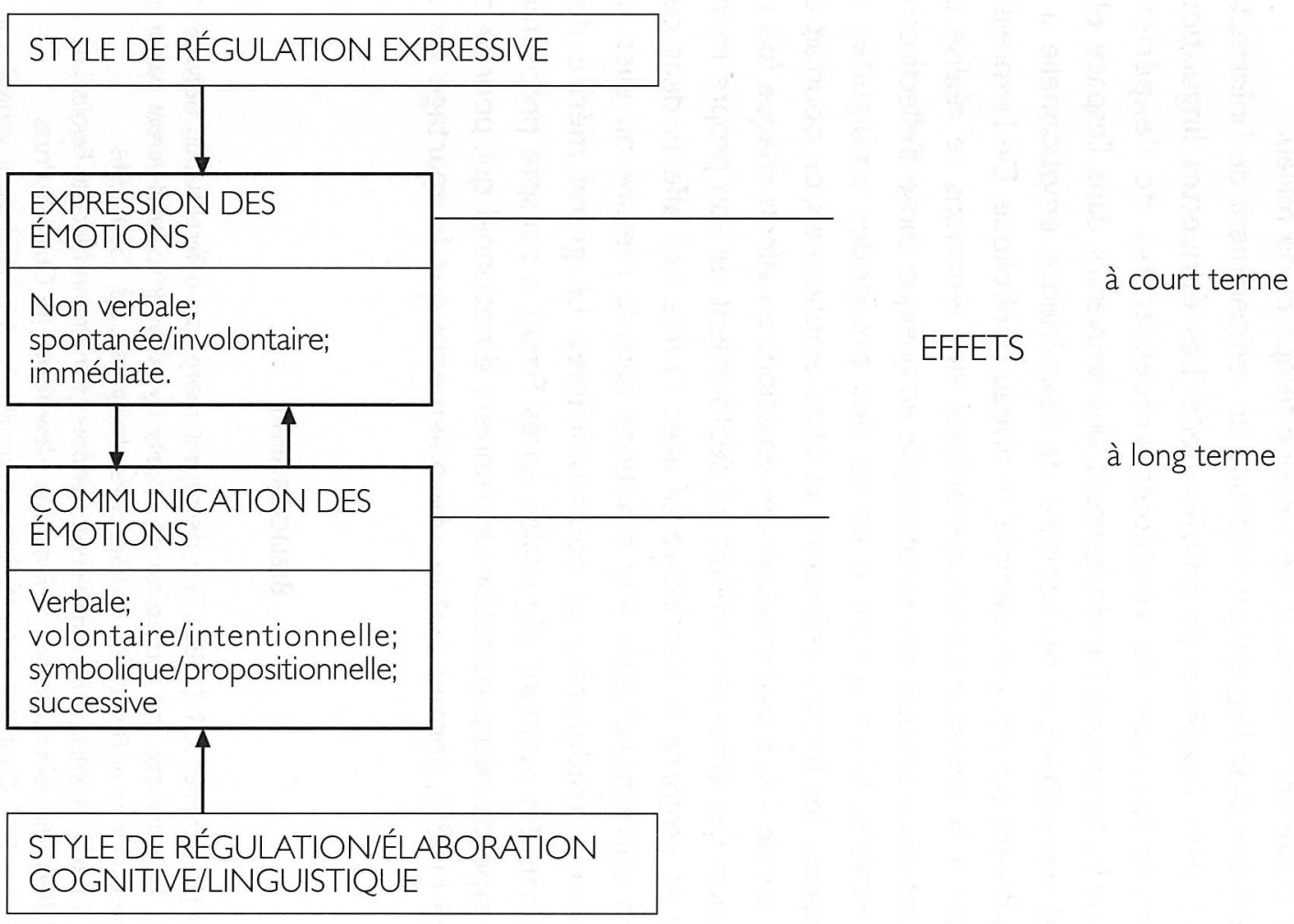

Figure 2 - Deux formes complémentaires d'extériorisation des émotions 
soit d'être excessivement bouleversé, soit de mettre une distance telle qu'il ne devient plus possible d'entrer en rapport avec l'autre. Donner un nom à ses émotions évite fondamentalement d'être écrasé au moment de leur impact, permet de créer des liens socio-affectifs et de retrouver, grâce à eux, une dimension commune de l'espace-temps et du milieu.

La catégorisation linguistique implique un remaniement de l'information contenue dans l'expérience émotionnelle. Les étiquettes linguistiques assument la fonction de véritables «réceptacles» de l'expérience émotionnelle, favorisent l'aménagement des souvenirs dans l'espace et le temps et permettent la reviviscence de l'expérience émotionnelle à un niveau différent qui est, précisément, le niveau symbolique. De l'expression immédiate à la communication symbolique des émotions se réalise une transformation de l'expérience émotionnelle en quelque chose d'effectivement communicable. Si ce n'était à cause des profondes ambiguïtés qui accompagnent les termes définissant les états émotionnels, on pourrait dire que l'on assiste à la transformation des émotions en affects chaque fois que l'élaboration des émotions prévoit un déploiement de son propre monde intérieur et renforce le lien interactif avec l'autre. Les affects dans cette acception correspondraient aux émotions dans la mesure où elles sont effectivement traduisibles et communicables. Le grand mérite de la psychanalyse des relations objectales, après Freud, a consisté précisément dans ce type de réappropriation de l'univers émotionnel qui, pour le dire à la Rimé (1989), permet tant le «déroulement» que le «partage social».

\section{BiBLIOGRAPHIE}

Asendorpf J.B., Scherer K.R. (1983). The discrepant repressor: differentiation between low anxiety, high anxiety, and repression of anxiety by autonomic-facial-verbal patterns of behaviour. Journal of Personality and Social Psychology. 45, pp. I334- 1346.

Bion W:R: (1962). Learning from Experience. London: Heinemann Medical Books Ltd.

Buck R: (1984). The communication of Emotions. New York: The Guilford Press.

Buck R., Miller R.E., Caul W.F. (1974). Sex, personality and physiological variables in the communication of affect via facial expression. Journal of Personality and Social Psychology. 30, pp. 587-596.

Buck R., Savin V.J., Miller R.E., Caul W.F. (1972). Communication of affect through facial expression in humans. Journal of Personality and Social Psychology. 23, pp. 362-37l.

Byrne D. (1964). Repression-sensitization as a dimension of personality. In: B.A. Maher (Ed.) Progress in experimental personality research (vol. I), New York: Academic Press. 
Darwin C. (1872). The Expression of Emotions in Man and Animals. London: Murray.

Ekman P. (1972). Universals and Cultural Differences in Facial Expressions of Emotion. In: J.

Cole (Ed.), Nebraska Symposium on Motivation 1971, Lincoln, Nebraska University Press.

Ekman P., Friesen W.V. (1969). The Repertoire of Nonverbal Behavior. Semiotica. I, pp. 49-98.

Eysenck, H.J. (1957). Dimensions de la personnalité, Paris: P.U.F.

Field T., Walden T. (1982). Production and perception of facial expressions in infancy and early childhood. In: H. Reese, L. Lipsitt (Ed.), Advances in child development (vol. 16), New York: Academic Press.

Frijda N.H. (1986). The Emotions. New York: Cambridge University Press.

Izard C.E. (197I). The face of emotion. New York: Appleton-Century-Crofts.

Izard C.E. (1977). Human Emotions, New York, Plenum Press.

Jones H.E. (1935). The galvanic skin response as related to overt emotional expression. American Journal of Psychology. 47, pp. 241-25I.

Laird J.D. (1974). Self-attribution of emotion: the effects of expressive behaviour on the quality of emotional experience. Journal of Personality and Social Psychology. 29, pp. 475-486.

Lanzetta J.T., Cartwright-Smith J., Kleck R.E. (1976). Effects of nonverbal dissimulation on emotional experience and autonomic arousal. Journal of Personality and Social Psychology. 33, pp. 354-370.

Lanzetta J.T., Kleck R.E. (1970). Encoding and decoding of nonverbal affect in humans. Journal of Personality and Social Psychology. 16, pp. $12-19$.

Lazarus R.S. (1966). Psychological stress and coping process. New York: McGraw-Hill.

Marty P., M'Uzan M. de (1963). La pensée opératoire. Revue Française de Psychanalyse. 27 (numéro spécial), pp. | 345- 1356.

Notarius C.I., Levenson R.W. (1979). Expressive tendencies and physiological response to stress. Journal of Personality and Social Psychology. 37, pp. 1204-1210.

Notarius C.I., Wemple C., Ingraham L.J., Burns T.J., Kollar E. (1982). Multichannel responses to an interpersonal stressor: interrelationships among facial display, heart rate, self-report of emotion and threat appraisal. Journal of Personality and Social Psychology. 43, pp. 400-408.

Nowicki S., Duke M.P. (1974). A locus of control measure for non-college as well as college educated adults. Journal of personality assessment. 38, pp. I36-137.

Pennebeker J.W., O'heeron R.C. (1984). Confinding in others and illness rates among spouses of suicide and accidental-death victims. Journal of Abnormal Psychology. 93, pp. 473-476.

Plutchik R. (1980). Emotion: A Psychoevolutionary Synthesis. New York: Harper and Row.

Ricci Bitti P.E., Gremigni P. (1995). Regolazione delle emozioni e rischio cardiovascolare. In: M. D'Alessio, P.E. Ricci Bitti, G.Villone Bettocchi (a cura di), Gli indicatori psicologici e sociali del rischio (pp. 301-318). Napoli: Gnocchi.

Ricci Bitti P.E., Gremigni P., Bertolotti G., Zotti A.M. (1995). Dimensions of Anger and Hostility in Cardiac Patients, Hypertensive Patients, and Controls. Psychotherapy and Psychosomatics. 64, pp. $162-172$.

Rimé B. (1989). Le partage social des émotions. In: B. Rimé et K.R. Scherer (direction de), Les émotions. Neuchatel: Delachaux et Niestlé.

Scherer K.R. (1982). Emotion as a Process: Function, Origin and Regulation. Social Science Information. 21, pp. 555-570.

Scherer K.R. (1984). On the nature and function of emotion: a component process approach. In: K.R. Scherer, P. Ekman (Eds.), Approaches to emotion. Hillsdale, N.J.: Erlbaum. 
Sifneos P.E. (1973). The prevalence of alexithymic characteristics in psychosomatic patients. Psychotherapy and Psychosomatics. 22, pp. 65-70.

Tomkins S.S. (1980). Affect as amplification: Some modification in theory. In: R. Plutchik, H. Kellerman (Eds.), Emotion, l: Theories of emotions. New York: Academic Press.

Tomkins S.S. ( 1 982). Affect Theory. In: P. Ekman (Ed.), Emotion in the human face. New York: Cambridge University Press.

Winnicott D.W. (1971). Playing and reality. London: Tavistock Publications. 


\section{Pedro Luzes}

Universidade de Lisboa

\section{L'INTERACTION COGNITION-ÉMOTION ET LA PSYCHOLOGIE CLINIQUE}

\section{INTRODUCTION}

Les émotions, en psychologie clinique, sont considérées comme des forces motivationnelles ayant plusieurs dimensions. Quelques auteurs y voient le plus important des systèmes motivationnels (S. Tomkins, 1970). Associées à d'autres systèmes psychiques, elles auraient la fonction d'amplifier ceux-ci de façon analogique par une sorte d'activation différenciée.

Les émotions sont comme un axe constitué par des phénomènes complexes qui sont soit comportementaux, (surtout, expressifs ou gestuels), soit physiologiques, (réactions neurovégétatives diverses), soit subjectifs (ressentis essentiellement avec tonalité agréable/désagréable). Autour de cet axe s'organisent d'autres systèmes psychiques, les attachements humains, la communication, la régulation de l'équilibre intrapersonnel (narcissisme).

Pour chaque état émotionnel, en clinique, les variations des différents phénomènes présents dans l'émotion semblent ne pas être en corrélation. Cependant certaines recherches montreraient une relation inverse entre composantes psysiologiques et expressives. Chez certains individus, dits extériorisants, la mimique émotionnelle serait vive et les réactions psychologiques faibles, chez d'autres classés comme intériorisants le contraire se vérifierait (E.H. Jones, 1960).

La théorie modulaire, que la psychologie clinique a adopté d'après le modèle établi par Freud et par la psychanalyse, suggère que la psyché est, comme le corps, composée de plusieurs «organes» qui se sont développés de façon à assumer des fonctions discrètes et individuelles (Fodor, 1983). Les émotions doivent se situer parmi les autres systèmes de motivation, et dans l'ensemble des systèmes psychiques. II y a la possibilité d'influences 
réciproques entre ces systèmes et les émotions et également la possibilité de conflits. Parmi ces systèmes qui sont en interaction ou conflit avec le système émotionnel, on compte le système perceptuel-cognitif.

\section{FONCTIONS DES ÉMOTIONS}

Dans Délire et rêves de la Gradiva de Jensen Freud écrit: «Nous demeurons en surface tant que nous ne parlons que de souvenirs et de représentations. S'il y a quelque chose de valeur dans la vie psychique ce sont plutôt les émotions (Gefühle): les forces psychiques ne sont significatives que si elles ont la propriété d'éveiller les émotions. Les représentations ne sont refoulées que parce qu'elles sont liées à des décharges émotionnelles (Gefühlsentbindungen) qui ne doivent pas avoir lieu. II serait plus juste de dire que le refoulement concerne les émotions, mais nous ne pouvons saisir celles-ci que par leur liaison (Bindung) à des représentations». (S. Freud, G. W., VII, 75, S.E., IX, 49).

Nous voyons dans cette citation Freud établir le contraste entre l'importance des émotions dans la vie psychique («s'il y a quelque chose de valeur... Ce sont plutôt les émotions») et d'autre part notre difficulté à prendre contact avec elles de façon substantive («nous ne pouvons (les) saisir que par leur liaison à des représentations»).

Comment considère-t-on aujourd'hui les fonctions des émotions?

Je distinguerai fonctions principales et secondaires. Les fonctions principales représentent l'action directe de l'émotion, les fonctions secondaires celles qui sont médiatisées à travers d'autres systèmes psychiques, ou en association avec eux.

Parmi les fonctions principales des émotions, on compte l'organisation

172 de l'attachement décrite par J. Bowlby (1960) et Mary Ainsworth (1978). II s'agit d'assurer la persistance de liens entre l'enfant et sa mère ou une personne qui la remplace. S'il y a perte ou danger de perte de liens d'attachements, les émotions de peur, d'angoisse, font leur apparition et assurent le retour à une situation de protection, le rétablissement des liens sécurisants.

Les émotions dans le développement précoce se montrent non seulement comme organisateur unipolaire mais également bi-polaire. Après l'attachement, les émotions aident à la création du clivage (Spaltung) des 
objets en bons et mauvais. II s'agit ici de la première rupture de la vie psychique, rendue en partie possible par une maturation cognitive et par un processus d'apprentissage, mais plus largement produite par les expériences émotionnelles.

Le plaisir joue un rôle dans l'émergence du bon objet mais il n'est pas suffisant en lui-même, les traces mnésiques qu'il laisse derrière lui ne sont pas assez durables pour assurer la constitution du bon objet. II faut y ajouter l'expérience, chez l'enfant, d'états affectifs positifs liés à des expériences d'amour et de tendresse, et de joie, la réception de louanges ou de conforts à un moment inattendu, ainsi que la possibilité d'exprimer librement ses désirs avec la compréhension des adultes. À ceci il faut ajouter des conditions de milieu fonctionnant comme un système de vie qui intègre le nouveau sans surcharges psychologiques, qui permet des pauses pour la reprise de forces, où les tensions exagérées sont absentes, où il y a pas de stress psysiologique ou psychologique. Ces conditions produisent des émotions de joie, de bien être, de bonheur, le sourire. Parallèlement naitrait le bon objet, comme imago.

L'expérience affective impliquant des abandons, des punitions, la frustration, la privation de plaisir, un rythme de vie irrégulier, avec tension, stress, provoque des émotions négatives de manque de sécurité, d'angoisse persécutive, d'agressivité, de honte, sur lesquelles s'appuie la constitution de l'imago du mauvais objet.

Par la suite il y aura une synthèse des imagos du bon et du mauvais objet. Dans la développement l'enfant prend conscience que les objets mauvais sont également les objets bons, et il aborde de façon plus directe leurs aspects effrayants ou laids. La connaissance des aspects négatifs renforce le caractère aimable et bienfaisant de ce qui est bon. La difficile synthèse du bon et du mauvais objet (position dépressive de Melanie Klein) permet l'interpénétration des émotions qui leur sont associées, produisant une gamme de nouvelles émotions: émotions de nostalgie, de curiosité pour l'ensemble du monde, culpabilité, angoisse, attitude réparatrice par rapport à l'objet.

Comme la création du bon et du mauvais objet, le complexe d'Oedipe introduit une bi-polarité dans la vie psychique. Mais on serait loin de la vérité si on disait que, dans le passage d'un état bi-polaire à l'autre, le bon objet serait remplacé par le parent du sexe opposé et le mauvais objet par le parent du même sexe. 
Les émotions liés à la constitution des objets internes, bons et mauvais, sont des émotions fondamentales qui apparaissent essentiellement comme réponse à des situations biologiques (faim-satieté, confort-inconfort, satisfaction-carence, bien-être-malaise). Dans le complex d'Oedipe nous nous retrouvons face à des interactions complexes entre l'enfant et ses parents, comportant des émotions très différenciées comme amour, rapprochement amoureux, agressivité, compétitivité, jalousie, espoir, déception.

La communication est une des fonctions principales des émotions. Cette fonction communicative était déjà signalée par Darwin: les mouvements qu'on retrouve dans toutes les émotions seraient conservés dans l'évolution pour aider la satisfaction de désirs ou pour éloigner des sensations pénibles (principe de la rétention des mouvements associés utiles). Parmi ces mouvements, quelques uns auraient, chez les espèces animales, une fonction communicative, constituant un langage avant le langage. Pour d'autres émotions il y aurait un recrutement de mouvements de caractère antithétique, organisant un langage ritualisé.

Spitz (1965) dans ses études sur la première année de vie de l'enfant a signalé trois organisateurs, pré-programmés, du développement psychique de l'enfant: le sourire du 3 e mois, l'angoisse du 8 e mois ou angoisse devant l'étranger et l'émergence des signes sémantiques et gestuels du «oui» et du «non». Ces organisateurs sont de nature émotionnelle, avec une signification communicative extrêmement importante, établissant également un langage avant le langage. Le sourire du bébé signe l'identification du bébé à la mère et sert leur communication interactive; l'angoisse du $8 \mathrm{e}$ mois sépare le moi du non-moi et permet l'identification du bon et du mauvais; l'émergence du «non» réaffirme l'identité du sujet et sa possibilité de s'opposer en tant que personne indépendance. II y a là aussi une première expression d'agressivité non-destructive.

En plus des fonctions primaires, on peut assigner plusieurs fonctions secondaires aux émotions. Ces fonctions secondaires s'organisent en association avec systèmes («modules») autonomes: pulsions, relations interpersonnelles, contrôle du milieu externe, équilibre narcissique. La conjonction des «modules» ensemble avec des catégories émotionnelles, correspondant à une croissante différentiation des états affectifs, me permet de proposer une grille pour les états affectifs [semblable à la grille de Bion, (1963) pour les pensées]. 
Les données suivantes sont nécessaires pour la lecture de la grille:

1) Dans les deux premières rangées horizontales on indique l'influence du plaisir et du déplaisir sur les différents systèmes modulisés. Le plaisir et le déplaisir sont des éléments présents dans toutes les émotions, sans être de vrais émotions (ce sont des proto-émotions, il leur manque les composantes comportementales et psysiologiques, et même psychologiques, des émotions). On pourrait parler ici d'une tendance à rechercher l'agréable et à éviter la douleur (principe de plaisir) au lieu de mentionner un plaisir (ou déplaisir) pur et simple. $\|$ y a des cas où l'influence du principe de plaisir est anti-adaptative. C'est le cas des contacts avec la réalité externe où la prédominance du plaisir (et des émotions positives) peut entraîner le sujet à oubli du réel. Si le déplaisir ou une un émotion négative inhibent en général les différents systèmes modulisés, un dosage modéré de plaisir peut entraîner un renforcement des pulsions, des rapports humains ou des rapports avec la réalité, comme moyen d'obtenir un meilleur contrôle sur le réel.

2) Les émotions primaires positives ou négatives, telles qu'elles ont été définies par S.S. Tomkins (1962, 1963), Izard (1978) et autres, occupent les deux rangées horizontales suivantes. Les caractéristiques principales des émotions primaires sont les suivantes: pré-programées, les stimuli qui les déclenchent sont détectés au niveau du système limbique, les réactions qui leur sont associées ont un pattern viscéral, de mimique faciale, et de vécu psychique qui est propre à chacune d'elles. Avec le développement, avec les processus d'apprentissage, les émotions primaires ne seront plus induites uniquement par le jeu des déclencheurs spécifiques, mais seront mises en marche par d'autres stimuli internes ou externes, par l'intervention d'autres affects, par la mémoire, par la pensée. Les rapports des émotions primaires avec les systèmes modulisées sont semblables, en apparence, à ce qui était décrit vis-à-vis des pré-émotions de plaisir et déplaisir. II y a cependant dans les émotions primaires d'importante mécanisme de rétroaction (feedback) avec réactions viscérales et musculaires venant du cœur, des vaisseaux, de l'intérieur du corps, des muscles faciaux, productions de sensations de froid ou de chaud. Les réactions périphériques 
tendent à reproduire de façon analogue les phénomène induite par les stimuli qui atteignent le système nerveux central (Tomkins 1978). Ainsi dans l'angoisse, ou dans la peur, où il y a la sensation d'un péril qui s'approche, l'accélération cardiaque, la rapidité de la respiration, la transpiration soudaine de la peau, le tremblement des mains, créent une sensation d'attaque semblable à celle produite par les événements extérieurs (incendie qui s'approche de nous, accident de voiture imminent). Ainsi la rétroaction des composantes périphériques des émotions maximise ce qui est ressenti au niveau central, soit dans le cas des émotions négatives, soit dans celui des émotions positives. Le négatif devient plus négatif, le positif plus positif.

3) Les émotions complexes sont le résultat d'un mélange d'émotions primaires ou de la transformation d'une émotion de base sous l'influence de mécanismes de défense. Quelques émotions complexes ont été déjà citées à propos de la situation oedipienne chez l'enfant et de la position dépressive, décrite par M. Klein. Tandis que les émotions primaires sont en nombre limité, le nombre d'émotions, complexes est pratiquement illimité. Les sentiments (ne pas confondre avec ce que les psychologues anglo-saxons appellent feelings) sont des émotions complexes. Parmi les sentiments il faut compter le doute, l'inquietude, la jalousie, le dépit, etc. Souvent les sentiments se rattachant à une tendance de la personnalité, se présentent alors avec une fréquence et un dynamisme augmentés (optimisme, réactions d'échec, dépression). Les sentiments à eux seuls ou ensemble avec les tendances de la personnalité sont infléchis ou modifiés par les mécanismes de défense (retournement dans son contraire, isolation, intellectualisation, inhibition de l'affect, formation réactionnelle ou inversion de l'affect).

4) Les scénarios intègrent les émotions avec les souvenirs d'états antérieurs de satisfaction ou de manque, retenus à partir des images laissées dans les systèmes intéroceptifs ou extéroceptifs. Ils peuvent servir comme récit mythique, des intellectualisations, qui nous aident à supporter les contrariétés du présent et à préparer l'avenir, par des projets d'action. Si les scénarios ne contiennent que les éléments historiques du passe, ou des représentations du réel actuel, sans 
émotions, ils deviennent des fictions froides, voire des délires. En créant des séquences, un récit, ils jouent un rôle important dans la genèse des émotions complexes. Dans les scénarios, les émotions et les images étoffent l'espace entre désir et monde extérieur évitant la répétition stéréotypée des conduites.

5) Les états psychosomatiques traduisent des émotions qui ont été conservées comme mémoire, au niveau du corps. Dans ces syndromes, il y a une difficulté à communiquer de façon langagière ou symbolique les composantes subjectives des émotions (alexithymia). L'incapacité à avoir une perception des états affectifs interviendrait négativement dans la régulation et la modulation des émotions et laisserait l'organisme vulnérable par rapport à une augmentation de la tension émotionnelle. Les phénomènes psycho-somatique plus que primitifs, seraient régressifs.

Pour une meilleure compréhension de notre «grille» il faut encore ajouter quelque données sur des émotions primaires, sur leur nombre et leur nature. Depuis la liste de Tomkins de sept émotions primaires, cinq négatives et deux positives, on trouve chez d'autres théoriciens des propositions légèrement différentes. On retrouve une révision partielle de ces listes dans le travail de G. Kirouac (1994). Je serais favorable à une liste plus ample que celle de Tomkins, par exemple à celle de R.S. Lazarus (|99|). II propose une liste de treize émotions. Parmi les émotions positives, il propose l'amour et l'émotion esthétique comme émotions primaires. En faveur de ces inclusions je citerais les observations de M. Klein (1952) qui a remplacé le conflit entre pulsions, ou entre pulsions et défenses, par un schéma génétique qui oppose amour et haine. Par ailleurs D. Meltzer (1988) suggère la présence d'un «conflit esthétique» dans les phases initiales du développement. Tout ceci serait en accord avec les récentes observations sur le nouveau-né, qui nous le signalent comme doué de fonctions affectives, cognitives, de mémoire et de motilité, hautement organisées capables d'interactions multiples avec la mère ou son substitut (E.V. Demos, 1992). En psychopatologie les émotions positives et négatives jouent un rôle capital, plus spécialement: l'amour, la haine (ou agressivité), l'angoisse, la tristesse. 


\section{COGNITION ET ÉMOTION}

G. Kirouac $(1989,1994)$ évoque le débat qu'oppose les tenants de l'indépendance relative des systèmes émotionnel et cognitif (Zajono, lzard) et les défenseurs de la position selon laquelle toute émotion aurait comme étape préliminaire une évaluation cognitive (Lazarus, Mandler). Les premiers théoriciens s'appuient sur le fait que les émotions auraient une priorité ontogénétique et psylogénétique et utiliseraient très peu les canaux de communication verbale. Les défenseurs de l'interdépendence de la cognition et des réactions affectives demandent comment il est possible de parler de réactions affectives à un stimulus, sans qu'il y ait eu au préalable, des phénomènes de connaissance. Mandler (1990) suggère que cette connaissance est une connaissance qui fait appel à des schèmes (semblables aux schèmes piagétiens) et produit une évaluation syncrétique.

Ma position est plutôt favorable à la relative autonomie du système émotionnel. Je me base sur l'expérience psychanalytique et sur les observations neurologiques de António Damásio.

Je commencerai par les observations de Damásio (1994) qui décrit un groupe de patients souffrant de lésions des lobes frontaux (plus spécifiquement des zones centrales et médianes bi-latérales). Ces patients ne présentent pas de phénomènes émotionnels, tout en gardant intactes leurs capacités intellectuelles, ceci devenant évident dans leur vie quotidienne et à travers les nombreux tests auxquels ils ont été soumis. Les capacités intellectuelles conservées ne leur sont cependant pas utiles, s'ils doivent s'adapter au réel, faire des choix, faire des plans pour l'avenir, justement parce que les émotions font défaut.

Tout en étant autonomes, les émotions sont comme les «qualificateurs» de tout ce qui se passe autour de nous. C'est comme si nous regardions un paysage à travers la fenêtre d'un train. Nous voyons, par une sorte de vision interne, un paysage constitué pas les organes du corps, en état de continuel changement, mais en même temps se juxtaposant à tout ce qui ne fait pas partie de notre corps.

Damásio (1995) nous parle aussi d'un cas d'un patient Boswell, qu'il suit depuis 1978. Ce patient présente des lésions du lobe temporel bi-latérales comme résultat d'une encéphalite. Le patient (et d'autres avec des atteintes anatomiques semblables) souffre d'une amnésie assez particulière. II vit dans un présent éternel et il n'arrive à retenir dans son esprit, quoi que ce soit, 
plus de 45 secondes. II ne reconnaît personne dans la clinique où il séjourne depuis des années, il ne sait pas si les personnes qu'il voit sont là depuis longtemps, ou si elles viennent d'arriver. II peut discuter du présent avec un interlocuteur. II acquiert des connaissances sensori-motrices, mais rien d'autre. Cependant l'amnésie ne l'empêche pas d'aller plutôt vers certaines personnes, qu'il paraît aimer, et d'éviter d'autres qui apparemment ne lui plaisent pas.

Damásio et son équipe l'ont testé pour concrétiser les préférences qu'il démontrait. Alors trois infirmiers qu'il rencontrait étaient instruits de façon différente: I'un l'accueillait chaleureusement et lui donnait des chocolats, des cigarettes, toutes les choses que le patient aimait; le deuxième était neutre; le troisième était négatif, disait «non» à toutes ses demandes et essayait de lui faire passer des épeuvres psychologiques extrêmement ennuyeuses. Par la suite, Boswell était confronté à une série de photographies où il y avait, parmi d'autres, les trois infirmiers. II disait préférer dans un haut pourcentage de cas l'infirmier «positif», dans un nombre moindre de fois l'infirmier «neutre», mais ne choisissait jamais la figure de l'infirmier «négatif». Tout ceci en ne pouvant, même de loin, identifier jamais qui que ce soit. Ses préférences étaient identiques après quelques mois, sans qu'il ait eu contact avec les trois infirmiers, mais pouvaient changer si le rôle attribué à un des infirmiers, par rapport au malade, changeait aussi. Damásio a montré ici ce qu'il considère «un apprentissage non-conscient basé sur une interaction purement émotionnelle».

\section{SUR ÉMOTION ET COGNITION: LES REPRÉSENTATIONS}

Freud distingue quatre variétés différentes de représentations: pulsionnelles, émotionnelles, épistémiques (ou de base), verbales. Dans un travail de 1915, Freud insiste, à propos des représentations de mot et de chose, sur le fait qu'il s'agit de formes distinctes de représentation, et non pas d'inscriptions différentes du même contenu dans les lieux psychiques différents (la même chose pouvant être dite pour les autres variétés de représentation).

Du point de vue génétique, les représentations affectives précédent les autres représentations. L'appareil psychique, initialement fonctionnant comme un appareil réflexe, est, à un moment donné, syntonisé sur les grandis besoins 
du corps (Freud, 1900). Les excitations produites par les besoins internes, captées par la sensibilité intéroceptive, conduisent à des décharges secrétoires ou motrices, laissant dans la mémoire une prédisposition mentale qu'on appellera quantum d'affect, représentant affectif, émotion. Ce représentant affectif sera le point de départ de signalisations et sera associé à des perceptions d'objet qui assurent la satisfaction (l'aliment, par exemple, qui sera représentation de chose). Les représentations pulsionnelles sont des éléments qui de façon innée, pré-programmée, sont liées aux pulsions et à leur satisfaction, semblables aux déclencheurs spécifique qu'on retrouve chez les espèces animales.

Les émotions ou d'une façon plus exacte les représentants affectifs, sont des «qualificateurs» (j'emploie ici le langage de Damásio) de tout ce qui se passe dans les autres systèmes. Par exemple, elles déterminent le tonus affectif, l'amplification, etc., des systèmes modulisés que nous avons établi dans notre «grille»: systèmes pulsionnel, de relations humaines, de relations avec la réalité extérieure ou sociale, de relations intra-subjectives.

Ce sont aussi les représentants affectifs qui à travers la projection sur des représentations de chose, permettront la formation de symboles et l'intégration de la réalité externe à notre réalité psychique.

Un cas de crise grave (psychotique) chez un jeune adulte, nous permettra de mieux saisir ces interrelations.

\section{Observation}

(Les lettres et chiffres renvoient à la «grille»).

A... homme de 22 ans, issu d'un milieu sócio-économique fortuné avec plusieurs frères. Les parents se sont séparés quand il avait dix ans. Le père qui dirigeait une entreprise industrielle était très autoritaire, la mère anxieuse, probablement déprimée, au moment de sa naissance.

La maladie débute quand $A$... a 19 ans. Ayant commencé à travailler dans une usine de son père, il devient convaincu que les communistes (ceci avant la chute de l'URSS) conspiraient contre le père (SC3). Un jour il a la certitude que son père est mort et le voit même dans un cercueil, à l'église. II fuit, il erre on ne sait pas très bien où, il brûle ses papiers d'identité. Comme il est délirant, on l'hospitalise, mais le malade refuse tous les soins. 
Après plusieurs séjours à l'étranger où on essais de lui faire suivre un traitement, il m'est amené pour psychanalyse. II vient accompagné par un infirmier ou par sa mère. II ne montre aucune conscience morbide, il me parle des communistes qui sont partout et du besoin qu'il a d'aider son père qui est maigre, souffrant (SC2).

Après l'avoir écouté une ou deus séances, je suis convaincu qu'il a éprouvé une grave déception (EC4) avec son père, qu'après l'âge de dix ans, il ne voyait que de loin (séparation des parents). Le contact plus récent avec le père a diminué les clivages que séparaient les aspects idéalisés (E2) du père de ses aspects négatifs (-E2). Cela a induit une dépression (EC4) qui fut suivie de nouveaux clivage associées à un essai de destruction de la mauvaises imago du père (hallucination de sa mort (SC3)).

Sa mère part à l'étranger après le début de son analyse. Le patient est angoissé (-E2) mais le nie. Au cours d'une des séances qui suit ce départ, il me parle de ses séjours en Afrique, où il aimait chasser les vautours avec un grand fusil (SC3). Je lui dis qu'il est devenu plus proche de moi (E2) avec le départ de sa mère, mais cependant il craint de n'avoir de moi que soins mercenaires (moi comme possible vauteur). Etant très dépendant (E4), mais en même temps tout-puissant (-E4), il doit protester très fort (EC2). II pose son portefeuille sur mon bureau, pour démontrer sa confiance (EC2) dans mon honnêteté. II commence à partir de là à me poser beaucoup de questions, 'quels voyages faites vous?' 'vos parents sont-ils encore vivants?'. En peu de temps, il arrive à la description des psychiatres qui l'ont soigné à l'étranger. II y avait un grec avec des moustaches, un français, un suisse - c'étaient de vrais vautours.

II s'est senti très malheureux (EC4) à l'étranger et il voulait rentrer à la maison. II était comme une pierre tombée au fond du lac (sa clinique se trouvait au bord d'un grand lac). II a formé une coquille autour de ses secrets - une coquille avec des épines. II ne leur a rien dit de ses secrets. À moi non plus, il ne pourra rien dire de ses secrets...

Dans la séance suivante, il est de mauvaise humeur, il se plaint de sa mère qui veut tout contrôler, qui pose tout le temps des questions, et ensuite va raconter les histoires de ses enfants à des «escrocs» (c.a.d. les psychiatres, le beau-père, moi).

Donc dans ces séances il s'est rapproché de moi par curiosité (EI), curiosité clivée du reste de sa personnalité, puisqu'il affichait de l'indifférence à mon égard. Ensuite il y a un deuxième clivage qui se produit me transformant en quelqu'un de semblable à un vautour. 
Le problème est le suivant. Comme tous les patients schizoides (V.Fairbairn et autres) A... a plus peur de son amour que de son agressivité. Il y a en lui un vautour qui souhaite pénétrer le corps de sa mère (et le mien car maintenant je remplace sa mère), ceci en raison de son désir de «rapproché» qui est devenu insoutenable. À ce propos, je me rappelle que sa mère avant de partir en voyage, s'était plainte des intrusions constantes de A..., qui veut être au courant de tout ce qui se passe dans sa vie, qui entre sans arrêt dans sa chambre, ou d'autres pièces où elle peut se trouver à la recherche d'un peu de solitude. La réaction de A... à son propre mouvement intrusif est paradoxale: il devient claustrophobe. II a peur de se noyer, d'être aspiré par le lac-mère (devenir comme une pierre tombée au fond d'un lac). Après avoir voulu dévorer, il a peur d'être dévoré. De là le besoin d'épines, qui représentent son agressivité contre ceux qui s'approchent de lui, son autisme, qui alterne et sert de protection contre une proximité trop grande. (II y a à coté des émotions signalées la formation de tout un symbolisme : lac, pierre, coquille, etc.). Nous voyons ici combien l'affect est proche de la représentation. Le patient A... ne peut pas se laisser pousser par les motivations qui sont en lui parce qu'elles comportent des émotions insoutenables. L'angoisse, la peur panique, la dépression, imposent de continuels changements : approximation, fuite, par exemple. Mais s'il peut fuir les médecins, le beau-père, il ne peut pas fuir ses parents. Donc il doit employer des mécanismes de défense comme clivage, identification projective (dans d'autres cas, ce serait peut-être le refoulement). Avec ces mécanismes de défense, il peut éliminer certaines représentations, certains imagos. Mais les émotions associées ne se laissent pas gommer, et pour un patient comme celui-ci, il faudrait pour y arriver un clivage multiple du moi (fragmentation, morcellement du moi).

\section{ÉMOTION ET PENSÉE}

On ne peut être d'accord avec Descartes aujourd'hui, lorsqu'il considère que, chez les hommes, les mêmes «passions... incitent et disposent leur âme» et «préparent leur corps» (Traité des passions, art. 40). La «préparation des corps», qui naît presque avec la vie, est fruit des émotions primaires ou fondamentales; tandis que l'incitation et la préparation de l'âme (c.a.d. les pensées) sont précédées par une modification des émotions, qui passent de primaires à secondaires. 
La forme primordiale de pensée, existant avant l'interaction pensée émotion-complexe, correspond à ce que Freud appelait processus primaire (repris par Levy-Bruhl sous le nom de mentalité primitive et par Piaget sous le nom de pensée égocentrique ou autistique). La fonction de cette variété inconsciente de pensée est de créer les images du rêve (à travers la condensation, le déplacement et la symbolisation) participant aussi à la formation des symptômes psychopathologiques, des mots d'esprit, etc. Le processus primaire se constitue comme une pensée orienté vers un but, mais qui pour aller vers ce but procède de façon syncrétique et magique, avec manipulation des représentations.

Avec l'installation du processus secondaire, une pensée, plus volontaire, plus consciente, s'établit, cherchant à faire la liaison entre les représentation selon des critères fonctionnels, et non plus seulement à travers une identité perceptive. En apparence, la pensée commandée par le processus secondaire est objective, véridique. En réalité, elle subit des influences du systèmes émotionnel qui infléchissent souvent les processus secondairisants de pensée. On peut citer comme transformations émotionnelles des pensées les exemples suivants:

I) L'absence d'émotions prive les pensées d'éléments essentiels pour l'orientation vers un but et pour la prise de décisions rapides (cf. les recherches neurobiologiques de Damásio, 1994).

2) À travers la formation de symboles (ce terme pris dans le sens de F. de Saussure et des psychanalystes), chaque pensée signifie ce qu'elle signifie en apparence et, en plus, a une signification symbolique, latente ou inconsciente, déterminée par le vécu du corps, par les antécédents relationnels de l'individu. L'influence d'émotions positives favorise la formation de symboles. Les émotions négatives gênent la formation de ceux-ci - par exemple, une forte et persistante angoisse peut paralyser le développement cognitif de l'enfant à travers des difficultés de symbolisation (M. Klein 1930).

3) Ce qu'on appelle scénarios (cf. grille) implique le recrutement de nouvelles réponses émotionnelles, en rapport avec les expériences du passé. Ce que nous pensons sur une personne, sur une situation, est modifié en fonction des expériences affectives du passé (relation 
transférentielle), les actions étant aussi modifiées. Les scénarios qui tendent à se répéter sont ceux de plus forte répercussion affective, et qui n'ont pas eu une élaboration adéquate (compulsion de répétition).

4) Les mythes, les légendes, les rituels, sont des scénarios qui existent dans la culture, pour satisfaire une motivation émotionnelle. Par la suite, ces scénarios organisent les pensées de l'individu, facilitant les relations sociales et l'établissement d'une identité de groupe (Luzes, 1969, cf. surtout le chapitre sur les Idéologies).

Les émotions altèrent le cours des pensées - celà est répété depuis longtemps - mais l'inverse est aussi vrai. La pensée abstraite, verbale, loin des éléments sensuels des choses, peut impliquer une détresse affective pour de nombreux sujets. Très souvent la pensée conceptuelle est imposée par les aspirations de l'idéal-du-moi, par la contrainte des adultes. Là où le sujet cherche à saisir la chose-en-soi, il se trouvera devant une non-chose, des flatus vocis, des systèmes incompréhensibles. On s'acheminera ainsi vers l'émotion dérégulée dont parlent R. Jouvent et S. Carton.

Chez les névrosés (soit hystériques, soit obsessionnels, soit phobiques), on se trouve devant un déficit affectif, résultat d'une défaillance face au réel. Le réel se montre d'autant plus difficilement assimilable en termes affectifs, par ces patients, qu'il n'est pas enrichi par une large variété de contenus symboliques.

On pourrait décrire un moi-affectif (lie aux émotions) face à un moi-cognitif ou même à un moi-social (lie aux règles, aux valeurs). Si ces «moi»»là (ou ces parties de moi) sont susceptibles de s'harmoniser entre-eux, ils peuvent également enter en conflit, du fait de leurs origines temporelle, ontogénétique, voire phylogénétique, différentes.

\section{RÉSUMÉ}

Les émotions, en Psychologie Clinique, sont considérées essentiellement comme des forces motivationnelles. En tant que dynamisme motivationnel, l'émotion amplifie toutes les autres motivations, organisant, par exemple, l'attachement et autres relations humaines, la communication, la connaissance du réel (plus spécialement à travers une médiation symbolique), le narcissisme. 
Tout en étant des organisateurs unipolaires (de l'attachement, par exemple), les émotions montrent à travers le clivage du «bon» et du «mauvais» objet, à travers la triangulation oedipienne, une capacité de bi-polarisation, liée à l'existence d'états affectifs positifs et négatifs.

On présente une «grille» qui permet l'association de plusieurs catégories émotionnelles avec d'autres systèmes psychiques (présentés comme modules).

GRILLE POUR LES ÉTATS AFFECTIFS

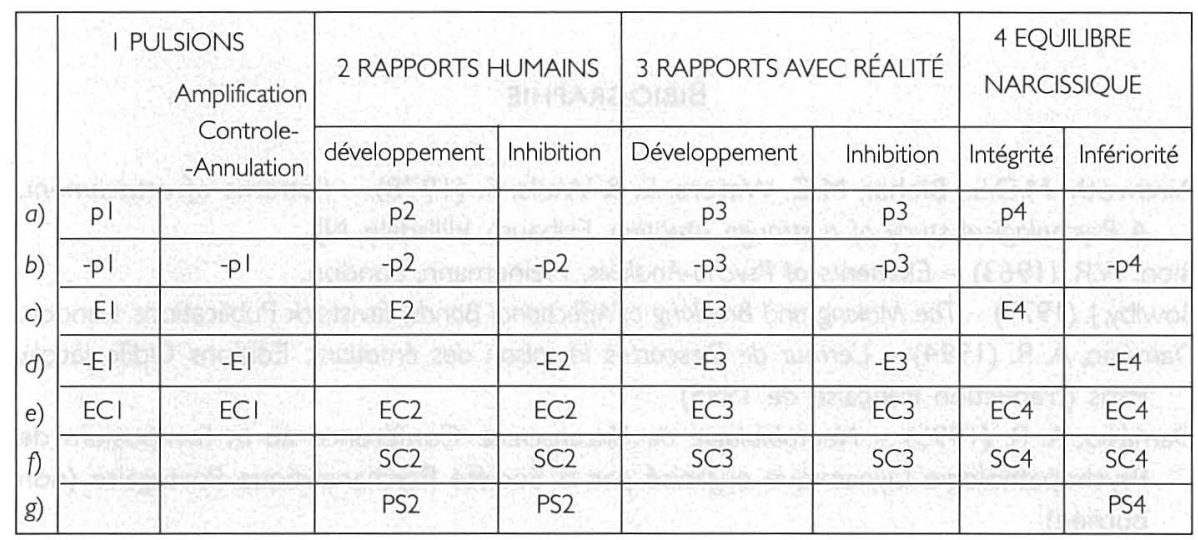
a) $P=$ Plaisir
b) $-p=$ Déplaisir
c) $\mathrm{E}=$ Emotions primaires positives
d) $-E=$ Emotions primaires négatives
e) $\mathrm{EC}=$ Emotions Complexes
f) $\mathrm{SC}=$ Scénarios
g) $P S=$ États psychosomatiques

En ce qui concerne les relations des systèmes émotionnel et cognitif, à côté des auteurs qui défendent la relative autonomie des deux systèmes, il y en a d'autres qui prônent la dépendance de l'émotion par rapport à la cognition, qui apparaîtrait toujours comme étape préliminaire de l'émotion. L'auteur défend la position autonomique (relativiste), en s'appuyant sur les observations psychanalytiques et sur les recherches de A. Damásio. Certains patients, tant ceux qui sont gravement dissociés psychiquement, que certains cas neurologiques, se montrent capables d'évaluations affectives, qu'ils modifient par apprentissage, de façon inconsciente, sans participation du système cognitif. On présente un cas personnel de décompensation 
psychotique, avec altérations d'origine affective des représentations psychiques (sans base cognitive reconnaissable).

Les relations entre cognition et émotion sont finalement considérées d'un point de vue moins figuratif et plus opératif. Ainsi on examine les influences de l'émotion sur la pensée. La pensée ayant des origines onto - et phylogénétiques différentes de l'émotion peut provoquer une disrégulation émotionnelle, s'associant dans les cas de névrose à un essai de supprimer les émotions à travers des mécanismes de défense.

\section{BIBIOGRAPHIE}

Ainswoth, M.D.S., Blehar, M.C. Waters, E. \& Walls, S. (1978) - Patterns of attachment: A Psychological study of a strange situation. Erlbaum, Hillsdale, NJ.

Bion, W.R. (1963) - Elements of Psycho-Analysis. Heinemann, London.

Bowlby, J. (1979) - The Making and Breaking of Affectional Bonds. Tavistock Publications, London.

Damásio, A. R. (1994) - L'erreur de Descartes la raison des émotions. Editions Odile Jacob,

Paris (traduction française de 1995)

Damásio, A. R. (1995) - Neurobiologie de l'Inconscient. Conférence au IX Symposium de Psychopathologie Dynamique organisé par la Société Psychanalytique Portugaise (non publiée).

Demos, E. V. (1992) - The Early Organization of the Psyche, in Interface of Psychoanalysis and Psychology (J.V. Barron, M.N Eagle, P.I. Wolitzked eds.). American Psychological Association, Washington.

Descartes, R. (1694) - Traité des Passions de l'Ame in Oeuvres et lettres de Descartes. Bibliothèque de la Pléiade. Gallimard, Paris 1953.

Fairbairn, W. R. (1952) - Psychoanalytic Studies of the Personality. Tavistock Publications, London, 1962 (2nd impression).

Fodor, J. A. (1983) - The Modularity of Mind. Mit Press, Cambridge, MA.

Freud, S. (1900) - Die Traumdeutung, G.W. II - III, S. Fisher Verlag, Frankfurt a. M.; S.E., V. Hogarth Press, London.

Freud, S. (1906) - Der Wahn und die Träume in W. Jensens Gradiva. G. W., VII; S.E., IX.

186 Freud, S. (1915) - Das Unbewusste. G.W. X; S.E. XIV.

Izard, C. E. (1977) - The Human Emotions. Plenum Press, New York.

Jones, E. H. (1960) - The longitudinal method in the study of personality in Personality Development in Children (I. Iscoe, H.W. Stevensoon, eds.). University of Chicago Press, Chicago. Jouvent, R. et Carton, S. (1994) - l'émotion dérégulée in Traité de Psychopathologie (D. Widlöcher, ed.). PUF, Paris.

Kirouac, G. (1989) - Les émotions. Presses de l'Université du Québec, Sillery, Québec.

Kirouac, G. (1994) - Les émotions in Traité de Psychologie expérimentale, 2e Vol. (M. Richelle, J. Requin, M. Roberts, eds.). PUF, Paris.

Klein, M. (1930) - The importance of Symbol-formation in the Development of the Ego, in The Writings of Melanie Klein, Vol. I, Hogarth Press, London. 
Klein, M. (1952) - Some theoretical conclusions regarding the emotional life of the infant in The Writings of Melanie Klein, Vol. III, Hogarth Press, London.

Lazarus, R.S. (1991) - Emotions and Adaptation. Oxford University Press, Oxford.

Luzes, P. (1969) - Les troubles de la pensée en clinique psychanalytique. Rev. fr. psychanal. Tome XXXIII, p.p. 727-843.

Mandler, G. (1990) - A constructivist Theory of Emotion in (N. L. Stein, B. Leventhal, T. Trabasso eds.). Psychological and Biological Approaches to Emotion. L. Erlbaum, Hillsdale, NJ.

Meltzer, D. \& Willams M. H. (1988) - The Apprehension of Beauty. The Role of Aesthetic Conflict in Development Violence and Art. The Clunie Press, Scotland.

Spitz, R. A. (1965) - The first Year of Life, I.U.P., New York.

Tomkins, S. S. (1962-1963) - Affect Imagery and Consciousness. Vols I \& II. Springer, Mew York. Tomkins, S. S. (1970) - Affect as the primary motivational system, in Feelings and Emotions (The Loyola Symposium, M. B. Arnold ed.). Academic Press, New York.

Tomkins, S. S. (1978) - Script theory : Differential Magnification of Affects, in Nebraska Symposium on Motivation (H. E. Howe, R.A. Dunstbier, eds.). Univ. of Nebraska Press, Lincoln. 
(Página deixada propositadamente em branco) 
Lise Fillion

University of Miami

\title{
ÉMOTION, COGNITION ET IMMUNITÉ (*)
}

\begin{abstract}
RÉSUMÉ
La thèse d'un lien entre les émotions et la maladie est envisagée depuis longtemps. Qu'en est-il de sa démonstration scientifique? La psychoneuro-immunologie (PNI) propose un mécanisme permettant de soutenir cette thèse. Son but ultime est d'expliquer l'influence des facteurs psychosociaux sur la résistance des individus à la maladie. La plupart des travaux réalisés en $\mathrm{PNI}$ appartiennent au domaine du stress psychologique et empruntent un cadre théorique provenant des domaines de la psychologie sociale et de la psychophysiologie. Quelques études relèvent directement de la psychologie des émotions. En respectant une proposition de rapprocher les études sur le stress psychologique et celles portant plus spécifiquement sur les émotions, cette présentation vise à fournir une intégration de différentes recherches réalisées en PNI. Dans un premier temps, cette intégration fournit un cadre théorique et une démonstration empirique permettant de soutenir la thèse d'une relation entre l'émotion et l'altération du fonctionnement immunitaire. Suite à une brève présentation de la psychoneuro-immunologie et du concept stress-émotion, plusieurs études reliant les stresseurs, le stress-émotion et l'immunité sont ainsi présentées. Les résultats démontrent clairement une altération immunologique en réaction à différents types d'événements stressogènes, soit en milieu naturel ou en laboratoire. En plus des stresseurs aigus, la recherche a également démontré une modulation immunitaire chez des sujets exposés à des stresseurs chroniques. Quoique
\end{abstract}

${ }^{*}$ *) Cet écrit a été réalisé grâce au soutien financier du Conseil de recherches en sciences humaines du Canada, bourse post-doctorale 756-93-040I. 
l'immunomodulation ne soit pas toujours liée à la condition d'exposition, elle apparait souvent associée à l'intensité de la réponse stress-émotion. Les résultats soutiennent l'hypothèse d'un lien entre l'émotion et l'immunité. Dans un second temps, l'intégration des différents travaux vise à faire ressortir l'importance d'inclure des variables médiatrices, tels l'évaluation cognitive du stresseur et les modes de gestion, afin de mieux comprendre les différences individuelles observées dans les profils de réaction. Le présent travail se termine par l'illustration de la pertinence d'inclure l'évaluation cognitive dans les études en PNI.

\section{ÉMOTION, COGNITION ET IMMUNITÉ}

La psychoneuro-immunologie (PNI) est souvent définie comme une extension de la recherche sur le stress, car son but ultime est d'expliquer l'influence des facteurs psychosociaux sur la résistance des individus à la maladie. En respectant une proposition récente de rapprocher les études sur le stress et celles portant plus spécifiquement sur les émotions, une intégration de différentes recherches réalisées en PNI est effectuée. Ce travail de synthèse vise, dans un premier temps, à soutenir la thèse d'une relation entre l'exposition à un événement stressogène, un phénomène émotionnel et une altération du fonctionnement immunitaire. Dans un second temps, l'intégration des travaux fait ressortir la nécessité d'inclure des variables médiatrices, telles que certains facteurs cognitifs, afin de mieux comprendre les différences individuelles observées dans les profils de réaction. L'illustration de la pertinence d'inclure l'évaluation cognitive termine cette présentation.

190

\section{Considérations Préliminaires}

La première partie de ce texte présente les premiers éléments de notre démonstration. Certains secteurs de la psychoneuro-immunologie, les principaux paramètres immunologiques évalués dans ce type d'étude, ainsi que le concept stress-émotion sont présentés. Pour de plus amples détails concernant le domaine de recherche de la PNI, le lecteur dispose de plusieurs ouvrages de référence (Ader, 1981; Ader, Felten, et Cohen, 1991; 
Herbert et Cohen, 1993; Fillion, Kirouac, Lemyre et Mandeville, 1994; KiecoltGlaser, 1988, 1992; O'Leary, 1990; Weisse, 1992).

La psychoneuro-immunologie. La PNI constitue un secteur de recherche interdisciplinaire qui s'intéresse aux relations entre les systèmes nerveux, endocrinien et immunitaire (Ader et al., 1991). Un de ces objets d'étude concerne les processus biologiques liant le stress psychologique et l'immunité (Ader, 198I). Entre le stress et la santé, les études épidémiologiques, sociologiques ou psychologiques ont indiqué des relations temporelles entre l'occurrence de stresseurs et celle de maladies (Cohen, 1985; Delongis, Folkman \& Lazarus, 1988; Miller, 1988; Minter \& Patterson, 1978; Zegans, 1982). Pour expliquer le rôle des stresseurs psychosociaux sur la santé, la thèse de la $\mathrm{PNI}$ invoque notamment une diminution de la résistance de l'hôte, se manifestant par une altération du fonctionnement immunitaire (Jemmott \& Locke, 1984). La recherche dans ce secteur a connu un essor considérable au cours de la dernière décennie. Les travaux réalisés, brièvement discutés ultérieurement, ont permis d'établir un lien entre l'altération, fonctionnelle et quantitative, de l'immunité, lors de l'exposition à un stresseur aigu (un deuil, un divorce, un examen académique) ou de l'exposition à un stresseur chronique (le chômage ou le fardeau de soin associé à une maladie chronique d'un proche). Des études récentes ont également démontré la présence de modulation de ces indices immunitaires, lors d'exposition stressogène en laboratoire.

Pour expliquer cet impact immunitaire lors de stress psychosocial et afin de fournir une base biologique permettant de lier le stress et la maladie, la $\mathrm{PNI}$ propose un mécanisme d'action immunomodulateur, lié à la réponse neurohormonale de stress. Cette réponse de stress correspond principalement à l'activation du système sympatique-adrénomédullaire (SAM), à l'activation du système hypothalamopituitaire-adrénocortical (HPAC), ainsi qu'à l'activation d'autres systèmes endocriniens (Asterita, 1985; Dantzer et Kelley, 1989; Schneiderman et McCabe, 1989; Selye, 1950). Les deux principales composantes de la réponse de stress, SAM et HPAC, représentent également une part importante de plusieurs réponses émotionnelles spécifiques (Baum, Grunberg et Singer, 1992; Sinha, Lovallo et Parsons, 1992). Cette réponse neurohormonale est décrite comme un mécanisme physiologique médiateur entre les stresseurs, le stress psychologique et la maladie (Antoni, 1987). Ces deux types de réponses ont été abondamment étudiées et notamment associées à plusieurs 
modulations immunitaires concomitantes (Ader et al., 1991; Blalock et Smith, 1985; Dantzer et Kelley, 1989; Daruna et Morgan, 1990). De plus, des aspects neurophysiologiques, reliant directement les systèmes immunitaire et nerveux, proposent une communication réciproque entre ces deux systèmes (Ader et al., 199I; Livnat, Felten, Bellinger \& Felten, 1985 ).

Le système immunitaire. Le système immunitaire (SI) joue un rôle de défense et protège l'organisme humain contre les micro-organismes générateur d'infections ou d'autres substances susceptibles de perturber l'état de santé. Le SI coordonne un ensemble de cellules et d'activité diverses visant la neutralisation et l'élimination des antigènes (voir Roitt, 1992). D'une part, les lymphocytes B qui circulent dans le sang assurent l'immunité dite «humorale» par la production d'anticorps (dont sont les immunoglobulines $\lg A$, IgD, lgE, lgG, $\lg M$ ) ayant la capacité de détruire spécifiquement l'antigène. D'autre part, les lymphocytes $T$ assurent l'immunité dite «cellulaire» en produisant des cytokines (médiateurs solubles ayant une action pharmacologique) qui permettent la régulation de la réponse immunologique ainsi que la destruction spécifique de l'antigène. Selon leur fonction, les lymphocytes $T$ se subdivisent en deux groupes: ( 1 ) les cellules $T$ «helper» $(C D 4+)$ qui influencent de façon positive la réponse immunologique, et (2) les CD8+, cellules T qui induisent des fonctions cytotoxiques ou suppressives. Par ailleurs, les réponses immunologiques non-spécifiques sont effectuées, entre autres, par les cellules NK (Natural Killer) qui reconnaissent les altérations membraneuses de certaines cellules tumorales ou de cellules infectées par des virus. Par leur capacité cytotoxique, elles jouent un rôle dans la défense contre les cancers et les infections virales.

Mesures de la réponse du système immunitaire. Parmi toutes les composantes du SI, les indicateurs immunologiques les plus fréquemment utilisés en PNI se regroupent en deux catégories, soit les mesures

192 quantitatives et les mesures fonctionnelles. Parmi les mesures quantitatives se retrouvent: (I) le nombre (décompte) des lymphocytes T helper (CD4+) et des lymphocytes $T$ suppresseurs ou cytotoxiques (CD8+), (2) le ratio CD4/CD8, (3) la quantification des cellules NK (CDI6+, CD56+ ou CD57+), (4) le dosage des anticorps totaux (ou immunoglobulines totales), (5) le dosage d'anticorps spécifiques et, (6) la quantification de cytokines, telle I'Interleukine2 (IL-2). À partir d'échantillons de sang frais provenant de la circulation périphérique, les mesures quantitatives sont généralement effectuées in vitro et correspondent au décompte des différentes sous- 
-populations de cellules effectives du SI. Parmi les mesures fonctionnelles se retrouvent: (a) la blastogenèse, transformation des lymphocytes, en réponse à une stimulation non spécifique par des agents mitogènes telle la concavaline $\mathrm{A}$ (ConA) et la phytohémagglutine ( $\mathrm{PHA}$ ) qui permettent d'évaluer l'ampleur de la division cellulaire des cellules T, ou tel le pokeweed (PKW) qui sert à l'évaluation des cellules $T$ et des cellules $B$ en division, (b) la transformation des lymphocytes en réponse à une stimulation spécifique par des antigènes tel un fragment peptidique du virus d'Epstein Barr (EBV) ou celui de l'herpès simplex de type I (HSV-I), (c) l'évaluation de l'habileté des lymphocytes à synthétiser et à libérer des cytokines, ou (d) l'estimation de l'activité cytotoxique des cellules NK (ACNK) par la libération («relargage») du chrome radioactif de cellules cibles pré-marquées qui sont détruites. Lors de la blastogenèse, il est également possible d'effectuer un dosage de cytokines telle l'IL-2. L'estimation de la réponse blastogénique ainsi que celle de la sécrétion des cytokines témoignent de l'ampleur de la réponse du SI (Fillion, Belles-Isles, Lemyre et Roy, 1994).

Les mesures quantitatives permettent la quantification du nombre de cellules effectives du SI, les mesures fonctionnelles permettent l'évaluation de leur capacité à effectuer leur fonction de défense. La variété et la complexité de ces mesures ne représentent qu'une portion simplifiée de la réponse du SI (Kiecolt-Glaser, 1988). Dans une section ultérieure où seront présentées différentes études réalisées en $\mathrm{PNI}$, la sensibilité de ces différents paramètres sera discutée. Avant de présenter ces études, l'élargissement du construit de stress est introduit en respectant la proposition de rapprocher les études sur le stress psychologique et celles portant plus spécifiquement sur les émotions (Lazarus, 1991; Scherer, 1990).

Le concept stress - émotion. La définition du stress psychologique ne fait pas consensus au sein des écrits scientifiques. Le champ du stress psychologique est vaste et comporte plusieurs volets. Parmi les plus citées, la définition du stress psychologique, qui provient du modèle transactionnel proposé par Lazarus (1966, 1991, 1993) et ses collègues (Lazarus \& Folkman, 1984; Lazarus \& Launier, 1978), se centre sur la notion de «transaction» entre les demandes contextuelles de la situation et les ressources de la personne. Le stress est vécu, lorsque l'individu évalue son bien-être menacé et ses ressources insuffisantes. Par son caractère non spécifique, le concept de stress psychologique réfère généralement à un état de détresse global (Baum, Grinberg, et Singer, 1992), et correspond souvent au pendant 
psychologique de l'activation physiologique (Ursin, 1982). Le construit de stress psychologique se distingue essentiellement de celui de l'émotion, par sa durée et son intensité (Scherer, 1990). Conceptualisant le stress comme une expérience émotionnelle, Scherer (1990) suggère l'utilisation du concept stress-émotion. Selon cet auteur, le stress est défini comme une expérience émotionnelle prolongée et souvent qualifiée d'une intensité supérieure, variant selon les caractéristiques de l'événement ou selon les les composantes spécifiques de l'évaluation personnelle. Cette conceptualisation amène également Scherer à proposer le regroupement des études portant sur les expériences de stress au sein du domaine de la pychologie des émotions. D'une façon parallèle, et sans rejeter l'existence d'un construit unidimensionnel et global de détresse associée à un stresseur psychosocial, les récents écrits de Lazarus (1991, 1993) proposent d'orienter la recherche actuelle sur le stress, vers l'étude spécifique des émotions concomitantes.

Dans le but de soutenir la thèse d'une relation entre l'émotion et l'immunité, nous retenons la suggestion de regrouper ces deux différents champs d'études, celui du stress et celui de l'émotion, par l'utilisation du concept de stress-émotion, associé globalement à un état de détresse ou spécifiquement à une émotion négative. Ayant sommairement présenté le construit stress-émotion ainsi que les mesures du SI pouvant y être associé, considérons maintenant les résultats de certains travaux empiriques permettant de soutenir la thèse d'une relation entre le stress-émotion et l'immunité.

\section{Immunité et stress-émotion: démonstration empirique}

Cette seconde section présente sommairement quelques travaux réalisés en $\mathrm{PNI}$ qui ont permis l'établissement d'un lien, entre l'exposition à un stresseur et une modulation de l'immunité (Ader et al., 1991). Le modèle théorique, souvent adopté dans ces travaux, propose que l'exposition à un stresseur soit accompagnée d'un état de détresse (stress-émotion), lui-même lié à des modulations immunitaires (Herbert et Cohen, 1993). Un sommaire d'études réalisées en milieu naturel et un résumé d'études récemment effectuées en laboratoire sont respectivement présentés. La majorité des études relèvent de la psychologie sociale; quelques-unes des études réalisées en laboratoire appartiennent directement de la psychologie des émotions. 
Les stresseurs impliquant une séparation. Les premiers travaux réalisés en PNI portent sur le deuil et sur l'état dépressif souvent associé à cet événement de vie (Bartrop, Lazarus, Luckhurst et Kiloh, 1977; Irwin, Daniels, et al., 1987, 1988; Shleifer et al., 1983). Ces études impliquent généralement un devis longitudinal, parfois de type quasi-expérimental, constitué à la fois d'un groupe endeuillé et d'un groupe de comparaison. Les sujets sont parfois appariés sur une ou plusieurs caractéristiques socio-démographiques. Au niveau immunitaire, les données font ressortir la sensibilité de l'évaluation fonctionnelle des lymphocytes $\mathrm{T}$ (en réponse à la $\mathrm{PHA}$ et à la ConA) et celle de l'activité cytotoxique des cellules NK (ACNK). Ces types de réponse fonctionnelle du SI se voient diminués dans les semaines qui suivent l'événement. De plus, ces résultats correspondent aux effets immunossuppresseurs observés lors d'études animales, réalisées auprès de jeunes singes séparés de leur mère (Coe et al., 1988). Dans certaines études, l'exposition à ce type de stresseur est également associée à une élévation de cortisol (Irwin et al., 1987; Coe et al., 1988), suggérant ainsi une relation entre le fonctionnement du SI et les états dépressifs. Les résultats de l'ensemble de ces travaux appuient l'effet immunosuppresseur de la séparation. Toutefois, l'effet n'est pas présent chez tous les sujets exposés. La diminution de l'immunité est parfois restreinte aux sujets présentant un état dépressif supérieur.

II n'est d'ailleurs pas étonnant que la recherche ultérieure dans ce secteur s'éloigne de la recherche sur le stress, et s'oriente vers l'étude clinique des patients dépressifs (Heisel, Locke, Kraus, et Williams, 1986; Kronfol et House, 1989). Tout comme dans les études sur le stress, l'indice immunitaire le plus relié aux états dépressifs est à nouveau la blastogenèse à différents mitogènes (PHA, ConA, PKW). Toutefois l'altération de l'immunité lors de dépression n'est pas présente chez tous les sujets déprimés. Les résultats provenant de ces études cliniques sont parfois même incohérents. Certains chercheurs ne distinguent pas les sujets dépressifs des non-dépressifs par leur réponse immunitaire (Schleifer, Keller, Bond, Cohen et Stein 1989). D'autres obtiennent des résultats contradictoires avec les hypothèses d'immunosuppression associée à un état dépressif: les sujets dépressifs ayant une réponse à la ConA faible présentent un état moins sévère que les sujets ayant une réponse immunitaire normale (Darko, Wilson, Gillin, et Golshan, 1991). En discutant des relations entre la sérotonine, les catécholamines et la réponse immunitaire, il est suggéré (Udelman et 
Udelman, 1991), que l'incohérence des résultats chez les patients dépressifs peut être associée aux perturbations neuroendocriniennes spécifiques de cette maladie. Dans une analyse critique de la dépression et l'immunité, Weisse (1992) signale également l'impact de la maladie sur l'immunité, et précise que les patients dépressifs présentant une diminution de la réponse blastogénique sont généralement de type unipolaire. Tout en faisant ressortir l'importance de se restreindre aux états dépressifs non cliniques, ou aux états cliniques de type unipolaire, l'ensemble des travaux soutient la thèse d'une relation entre le deuil, l'état dépressif et l'immunité.

Les examens académiques et l'immunité. Un autre stresseur naturel, dont l'occurrence est facilement contrôlable ou prévisible, concerne les examens académiques. Cette situation constitue une transaction fréquemment étudiée en $\mathrm{PNI}$, et bénéficie d'une validité écologique reconnue. Utilisant des devis de recherche de type quasi-expérimental pré-test post-test, l'ensemble de ces études atteste d'un effet immunomodulateur lors d'une situation d'examens (Fillion et al., 1989; Glaser et al., 1986, 1990, 1993; Halvorsen, et Vassend, 1987; Kiecolt-Glaser et al., 1986, Workman et Lavia, 1987). Ces travaux démontrent la sensibilité des paramètres fonctionnels suivants: la blastogenèse en réponse à la ConA et à la PHA, le dosage de l'IL-2 et l'évaluation de l'activité cellulaire des NK (ACNK); ainsi que la sensibilité des indices quantitatifs suivant: le titrage d'anticorps spécifique au EBV, le nombre de cellules NK et de cellules CD8+. Ces études relient des stresseurs aigus à l'immunité, et appuient l'hypothèse d'un effet immunodépresseur associé au stress. Toutefois, l'immunomodulation n'est pas toujours présente et semble à nouveau liée à la réponse de stress-émotion. Afin d'expliquer ces variations individuelles, des caractéristiques psychologiques, incluant l'évaluation cognitive et les stratégies de gestion, sont proposées comme médiateur (Herbert et Cohen 1993; Pelletier \& Herzing, 1989; Vingerhoests \& Assies, 1991). Des résultats appuient l'importance des modes de gestion. La relaxation peut en effet atténuer les effets immunodépresseurs (Kiecolt-Glaser et al., 1986), alors que l'intrusion cognitive (Workman et Lavia, 1987) semble l'amplifier. Bien que proposée dans plusieurs discussions, l'évaluation cognitive n'est que partiellement, ou pas mesurée. Par ailleurs, l'effet immunomodulateur semble également varier selon la durée et la temporalité de la transaction, de même que selon le type de paramètre immunologique étudié (Fillion, Kirouac et al., 1994). Le manque de standardisation dans le moment des prises de mesures rend 
difficile la comparaison des résultats. L'étude de stresseurs chroniques peut contourner certains de ces problèmes.

Les stresseurs chroniques et l'immunité. Les stresseurs chroniques réfèrent à des situations perdurantes. L'infertilité, (Kedem, Bartoov, Mikulincer et Shkolnik, 1992), ta situation de fardeau familial lié à la maladie d'Alzheimer, (Kiecolt-Glaser et al., 1987), ainsi que le chômage (Arnetz et al., 1987, 1991) ont été associés à des diminutions graduelles de la réponse immunitaire. Malgré les faiblesses méthodologiques associées principalement aux devis corrélationnels, ces études appuient l'hypothèse d'immunodépression lors de stress. L' ensemble de ces résultats, ajoutés à ceux provenant des études réalisées lors d'exposition à des stresseurs naturels, aigus ou chroniques, soutiennent l'hypothèse d'un lien entre les états de stress-émotion et le fonctionnement du SI. Des différences individuelles sont toutefois observées. Parfois la modulation immunitaire n'est pas liée à la condition d'exposition, et est davantage associée à l'intensité de la réponse de stress-émotion. Pour expliquer cette différence d'intensité, l'inclusion de variables intermédiaires, tels l'évaluation cognitive et le mode de gestion, est proposée. Ainsi, pour qu'un stresseur affecte le fonctionnement du SI, les premières études réalisées en $\mathrm{PNI}$ amènent à formuler l'hypothèse conditionnelle de la prévalence d'un état de stress-émotion intense et prolongé.

Les stresseurs en laboratoire et l'immunité. Afin de vérifier cette dernière hypothèse ainsi que la présence de lien de causalité entre les stresseurs et l'immunité, des études en laboratoires sont réalisées. Suite à l'exposition à un stresseur, une réactivité immunitaire, quantitative et fonctionnelle, est observée (Bachen et al., 1992; Manuck et al., 1991; Naliboff et al., 1991). À peine quelques minutes suivant l'exposition au stresseur, les sujets manifestent une augmentation du nombre de cellules CD8 +, une élévation du nombre ainsi que de la fonction des cellules NK, et une diminution de la blastogenèse aux mitogènes ConA et PHA. La réaction observée est de fait très comparable à celle suivant une injection d'épinéphrine et fait ressortir le rôle prépondérant du système nerveux sympathique (SNS) (Kiecolt-Glaser et al., 1992), quoique l'amplitude de cette réactivité demeure associée à la sécrétion de cortisol (Cacioppo, 1994). Par ailleurs, malgré un patron cohérent d'effets immunitaires, des différences individuelles demeurent observables. Certains sujets réagissent très fortement. Les sujets qui manifestent une réponse importante au niveau immunitaire manifestent également une réactivité cardiovasculaire accrue (Manuck et al., 1991; Sgoutas et al., 1992; Stone et al., 1993; Zakowski et al., 1992, 1994). II devient 
de plus en plus évident que l'exposition à un stresseur affecte la réactivité immunitaire et la réponse cardiovasculaire de façon concomitante. Dans le domaine de recherche cardiovasculaire, l'étude des différences individuelles, présentée ultérieurement, fait également ressortir l'importance des variables psychologiques médiatrices.

Les études sur les émotions et l'immunité. Considérant que l'émotion est souvent conceptualisée comme un bref phénomène (Ekman, 1984), et considérant la rapidité de la réactivité immunitaire décrite dans les études sur le stress, la recherche liant l'immunité et l'émotion devient de plus en plus pertinente. Quoique peu nombreuses, l'induction d'émotion en situation de laboratoire a permis de réaliser que les émotions négatives ou positives sont associées à un profil comparable de modulations immunitaires fonctionnelles (Futterman et al., 1994; Knapp et al. 1992). L'amplitude de la réactivité immunitaire est plus prononcée lors d'émotion négative (Knapp et al. 1992) et est associée aux indices cardio-vasculaires d'activation du SNS (Futterman et al., 1994). Ces études sont cohérentes avec les études réalisées sur le stress et font ressortir que la réactivité immunitaire est également associée à l'induction d'émotions positives.

La recherche réalisée en laboratoire permet de lier la réponse neurohormonale de stress et le SI. De plus concernant I'hypothèse conditionnelle d'un état émotif intense et prolongé, la rapidité de la réactivité immunitaire tend à infirmer la notion de nécessité d'un caractère soutenu, justifiant en ce sens la recherche liant l'émotion et l'immunité. Toutefois l'association entre la réactivité immunitaire et l'activation du SNS appuie la condition d'intensité: les sujets hautement réactifs au niveau cardio-vasculaire manifestent également une réactivité immunitaire supérieure. La recherche en laboratoire sur l'émotion et l'immunité permet de préciser que l'amplitude de la réactivité cardiovasculaire est plus importante que la valence positive

198 ou négative de l'émotion. Par ailleurs, tout comme la recherche en milieu naturel, la recherche en laboratoire fait ressortir d'importantes différences individuelles dans les profils de réactivité immunitaire.

La première section de ce texte a permis la précision du construit de stress-émotion et la présentation sommaire des mesures du SI pouvant être associées. La seconde section a présenté des travaux empiriques permettant de soutenir la thèse d'une relation entre le stress-émotion et l'immunité. Cette dernière section propose l'inclusion de l'évaluation cognitive pour expliquer une part des différences individuelles observées. 


\section{Différences individuelles et évaluation cognitive}

Selon la perspective transactionnelle (Lazarus et Launier, 1978; Lazarus et Folkman, 1984), l'intensité et l'amplitude de la réponse de stress-émotion dépendent: $a$ ) de l'évaluation cognitive de l'enjeu de la transaction (potentiel menaçant ou dommageable du stresseur; première composante de l'évaluation cognitive), ainsi que $b$ ) de l'évaluation de la capacité à prévenir ou à gérer cette situation (seconde composante). Malgré un consensus sur l'importance d'inclure un médiateur cognitif dans les recherches sur le stress, le construit et la définition opérationnelle de l'évaluation cognitive demeurent à préciser (Monroe et Kelley, 1995).

Dans le domaine de la recherche sur le stress, l'évaluation cognitive a souvent été mesurée par l'intermédiaire d'un seul item portant sur la perception immédiate de l'individu (menace, défi, perte ou bénéfice) spécifique à un stresseur donné (Moos et Schaefer, 1993). Ce type de mesure est principalement utilisé dans les études réalisées en laboratoire et présente l'avantage d'évaluer directement et spécifiquement le construit en accord avec le modèle transactionnel. Malgré la pertinence de l'information donnée par ce type évaluation, le format restreint à un seul item peut présenter des limites psychométriques. De plus, l'information sur la validité, la stabilité et les caractéristiques spécifiques de ce type de mesure demeure restreinte (Monroe et Kelley, 1995). Pour évaluer le construit d'évaluation cognitive, une autre stratégie est proposée avec la recherche sur le stress, axée sur la mesure des événements de vie. Dans ce secteur de la recherche, le stress est associé au nombre (Holmes et Rahe, 1967) ainsi qu'aux caractéristiques contextuelles (Brown et Harris, 1978) des événements de vie ou des stresseurs. L'inventaire des événements de vie est généralement réalisé, soit par l'intermédiaire d'un questionnaire, ou soit lors d'une entrevue structurée. Afin de considérer les différences individuelles lors de l'estimation du nombre de stresseurs, l'inclusion de dimensions subjectives s'est graduellement ajoutée. Avec ce type de mesure, l'évaluation cognitive des dimensions d'impact (Horowitz et al., 1979; Sarason et al., 1978), de désirabilité (Rhodewalt et Zone, 1989; Vinokur et Selzer, 1975), d'importance (Parkes, 1986), de contrôle (Averill, 1973; Flannery, 1986; Folkman, 1984; Thompson, 1981), de menace (Lazarus, 1966; Mason, 1975), de prévisibilité, d'incertitude et d'anticipation (Paterson et Neufeld, 1987) a, tour à tour, été étudiée à titre de variables médiatrices, dans la relation 
entre la personne et l'événement stressant. Certaines de ces mesures permettent de distinguer les deux composantes de l'évaluation cognitive proposées par le modèle transactionnel de stress, d'autres pas. Afin de mieux représenter le construit, et d'en extraire les principales composantes, l'évaluation cognitive est parfois mesurée en regroupant plusieurs dimensions. Ainsi, Holm et al. (1986) identifient trois composantes, qui correspondent respectivement à l'Impact, le Contrôle et la Prédictibilité. Lemyre et ses collaborateurs $(1986,1993)$ identifient également un modèle incluant deux ou trois composantes: I'Impact, la Maîtrise et parfois, l'Incertitude. La variance de ce dernier facteur semble restreinte à certains stresseurs aigus (Lemyre et Tessier, 1995), variant possiblement selon le statut de résolution de l'événement; en d'autres circonstances, la contribution des items associés à ce facteur, demeure associée aux composantes Impact et Maîtrise (Fillion, 1993). Globalement, ces auteurs proposent de regrouper plusieurs dimensions de l'évaluation cognitive d'un stresseur afin d'identifier les composantes principales. Cette stratégie empirique permet l'identification d'au moins deux facteurs qui se rapprochent théoriquement du modèle transactionnel de stress et qui se dessine comme une avenue intéressante pour définir opérationnellement le construit. Bien que les démarches de validation de ce construit se poursuivent dans le domaine du stress, les récents travaux de Lazarus (Lazarus, 1991; Smith et Lazarus, 1993) proposent d'élargir le construit en précisant d'une part, le contenu des composantes, et d'autre part, la spécificité de ce contenu en fonction de différentes émotions.

Parallèlement aux travaux sur le stress, la précision du contenu et de la spécificité des composantes de l'évaluation cognitive constitue un objet d'étude en psychologie de l'émotion (Roseman, Spindel et Jose, 1990; Wallbott et Scherer, 1991). Dans le domaine des émotions, plusieurs théories cognitives ont été proposées afin d'élaborer une définition opérationnelle du construit de l'évaluation cognitive, applicable pour toutes les émotions (Arnold, 1960; Roseman, 1984, Smith et Ellsworth, 1985; Scherer, 1988). Pour définir ce construit, Arnold (1960) propose trois dimensions: conséquences (positive ou négative), contrôle (capacité à gérer) et occurrence (temporalité: est-ce présent en ce moment?). Roseman et al. (1984) en proposent cinq: enjeux de la situation, possibilité de contrôle, probabilité, lieu de contrôle et intérêt ou motivation; Scherer (1988) en propose également cinq: agrément, potentiel de maîtrise, nouveauté, rapport aux buts et accord avec les standards; enfin, Smith et al. $(1985,1989)$ en propose six: agrément, 
contrôle situationnel, incertitude, lieu de contrôle, attention et effort anticipé. Malgré certaines limites psychométriques des échelles utilisées pour mesurer ces dimensions de l'évaluation, des convergences entre ces théories permettent de soutenir la validité de convergence du construit. En comparant, au niveau de 16 émotions, le pouvoir prédictif de trois modèles nommés ci-haut (Arnold, Roseman et Scherer), Roseman et al. (1990) identifient des facteurs de convergence. Concernant des émotions facilement associables à l'état de stress, telles la détresse et la peur, les auteurs font ressortir les facteurs cognitifs suivants: les enjeux, le potentiel de maîtrise, l'occurrence, l'attribution et la motivation. Notons que les trois premiers facteurs sont facilement comparables aux composantes proposées en psychologie sociale. Tout en chevauchant théoriquement les deux principales composantes de l'évaluation cognitive proposées dans les modèles de stress, la définition opérationnelle de l'évaluation cognitive, au sein des théories de l'émotion, offre un potentiel accru de dimensions et propose un patron spécifique d'organisation de celles-ci, selon l'émotion. L'évolution respective de ces deux domaines de recherche, le stress et l'émotion, pourrait éventuellement déboucher sur la définition opérationnelle d'un seul construit d'évaluation cognitive. Ce construit unique inclurait un nombre exhaustif d'items permettant de représenter à la fois les deux composantes du modèle transactionnel, et à la fois le regroupement spécifique de différentes dimensions.

L'évaluation cognitive et l'immunité. Au sein de plusieurs théories, se retrouve l'inclusion d'un médiateur cognitif entre l'exposition à un stresseur et la réponse de stress. Selon le type de stresseur et notamment, selon la possibilité de contrôle perçu, un mode de gestion actif ou passif (inhibé) est déployé (Schneiderman et McCabe, 1989). Chacun de ces modes de réponse semble accompagné d'une modification hémodynamique et endocrinienne caractéristique. Au mode actif, correspondent notamment une augmentation du rythme cardiaque, une élévation de la tension artérielle systolique, une sécrétion de catécholamines et une dominance de la composante du SAM. Le mode passif ou inhibé se caractérise davantage par une réponse hémodynamique vasculaire, c'est-à-dire par une augmentation de la résistance périphérique des vaisseaux, et est également accompagné d'une sécrétion de corticostéroïdes témoignant de la composante HPAC (Schneiderman et McCabe 1989). Un profil mixte est également possible (Schneiderman et McCabe, 1989). Les émotions de peur 
et de joie semblent principalement associées à la composante SAM alors que l'émotion de colère apparait simultanément liée aux deux composantes (Sinha, Lovallo et Parsons, 1992). Dépendamment des théories, ce mode de gestion correspond à différentes appellations: «effort ou détresse» (Frankenhaueser, 1980), «lutte/fuite ou retrait» (Cannon, 1930), «combat actif ou défaite» (Folkow, 1993). Malgré l'importance accordée à l'évaluation cognitive au sein de de ces modèles, leur démonstration a été principalement réalisée par des études animales, excluant ainsi la possibilité de mesurer directement le construit. Même dans les études humaines, les recherches ont davantage porté sur les caractéristiques du stresseur ou les conditions contextuelles de l'expérimentation (contrôle, prévisibilité, compétition, harcèlement, consignes) pouvant potentiellement moduler l'évaluation cognitive et affecter la réponse neurohormonale de stress (Lazarus et Folkman, 1984). Si la recherche a permis de vérifier l'association entre certains types de stresseurs et le mode de réponse (Hurwitz et al., 1993; Zakowski et al., 1994), les dimensions cognitives n'ont toutefois que rarement, partiellement ou indirectement été évaluées (Monroe et Kelley, 1995). Ainsi malgré son rôle théorique prépondérant pour expliquer l'occurrence de différents profils de stress, l'hypothèse du rôle médiateur de l'évaluation cognitive ne dispose que d'appuis indirects, et demeure à vérifier.

Pour illustrer la pertinence d'inclure l'évaluation cognitive dans les études en $\mathrm{PNI}$, nous terminons par la présentation de nos travaux, réalisé à la fois en milieu naturel, lors d'un diagnostic de cancer (Fillion et al., 1993, 1995, 1996) et en laboratoire (Fillion, 1995).

Évaluation cognitive et immunité lors de d'un diagnostic de cancer. L'étude est effectuée auprès de 72 femmes rencontrées avant et après une chirurgie diagnostique. Suite au diagnostic de cancer du sein de stade précoce, 36 femmes sont appariées, sur 4 variables socio-démographiques, à 36 femmes recevant un diagnostic de tumeur bénigne. Les résultats suggèrent que la période d'anticipation du diagnostic est associée à un plus grand niveau de perturbation que celle suivant le diagnostic de cancer comme tel. Les patientes qui perçoivent plus d'Incertitude et associent davantage d'Impact au fait d'avoir le cancer semblent être celles qui manifestent les niveaux de stress les plus élevés, et les patientes qui présentent un meilleur sentiment de Maîtrise face au cancer et une perception pré-post cohérente concernant l'Impact de cette maladie semblent présentées une meilleure immunité fonctionnelle (PHA et ConA) et quantitative (NK). Les résultats confirment 
l'importance de l'évaluation cognitive du diagnostic pour expliquer les différences individuelles observées lors de l'adaptation psychoimmunologique au cancer du sein de ce stade.

Évaluation cognitive et immunité lors d'un stresseur en laboratoire. Cette seconde étude, réalisée cette fois en laboratoire, porte sur le rôle médiateur de l'évaluation cognitive, lors d'une exposition à un bref stresseur. Un groupe de sujets séropositifs au $\mathrm{VIH}(\mathrm{VIH}+)$, non diagnostiqué SIDA, est comparé à un groupe de sujets non infectés par le virus $(\mathrm{VIH}-)$. Le stresseur choisi consiste à préparer, ainsi qu'à livrer un exposé oral. Lors de cet exposé, le sujet est invité à se défendre verbalement contre une fausse accusation de vol à l'étalage. Des instructions guident la préparation ainsi que la présentation de l'exposé. Des travaux antérieurs ont permis d'observer une association entre la période d'exposition (préparation/exposé) et le mode de réponse: la période de préparation étant associée à un mode de gestion actif, alors que la période d'exposé verbal étant liée un mode mixte (Hurwitz et al., 1993). Le stresseur choisi permet ainsi l'occurrence potentielle des deux modes de réponses. Les mesures cardiovasculaires et immunitaires sont prises avant l'exposition, en période de préparation, en période de présentation et en période de récupération. La mesure cognitive est administrée immédiatement après la période de récupération, avant le retrait des électrodes, du brassard et du cathéter veineux. Pour l'ensemble des sujets, une association négative entre la perception de Maîtrise et la réactivité physiologique est observée. En ce qui concerne la réactivité immunitaire, plusieurs indices de la réactivité immunitaire quantitative (augmentation du nombre de leucocytes et de lymphocytes totaux, et du nombre absolu de cellules $\mathrm{CD} 8+$ ) et fonctionnelle (augmentation de l'activité cytotoxique des cellules NK(ACNK) ) sont négativement corrélés avec la perception de Maîtrise. Un profil différent, voire même opposé, est observé selon le groupe, en ce qui concerne la relation entre la perception d'Impact et la réactivité. Chez le groupe HIV+, plusieurs indices de la réactivité cardiovasculaire et immunitaire quantitative (augmentation du nombre de leucocytes et de lymphocytes totaux, et du nombre absolu de cellules CD4+) se retrouvent négativement associés à la perception d'Impact. Les résultats confirment la présence d'associations significatives entre l'évaluation cognitive et la réponse de réactivité physiologique. Les résultats observés chez l'ensemble des sujets appuient les modèles théoriques voulant qu'une perception de contrôle (Maîtrise) affecte le mode de réactivité quoique l'observation attentive des 
résultats observés chez les sujets $\mathrm{VIH}+$ laisse envisager un rôle médiateur moins important à la perception de contrôle et fait davantage ressortir l'importance de la perception d'Impact négatif. Concernant cette dimension de l'évaluation cognitive (Impact), les relations observées chez les sujets de ce groupe $(\mathrm{VIH}+)$, présentent une direction opposée à ce qui devrait théoriquement être observé. Plus le sujet infecté perçoit la situation menaçante, moins il semble réagir au stresseur, alors que l'opposé est observé chez le groupe $\mathrm{VIH}$-. [Les résultats sont discutés en fonction de la théorie du syndrome d'adaptation et en fonction de l'évolution de l'infection $\mathrm{au} \mathrm{VlH}$ ]. Ces résultats s'ajoutent à ceux de l'étude précédente et confirment la contribution significative de l'évaluation cognitive dans l'explication des différences individuelles observées dans les profils de réactivité immunitaire.

\section{CONCLUSION}

En respectant une proposition de rapprocher les études sur le stress et celles portant plus spécifiquement sur les émotions, une intégration de différentes recherches réalisées en PNI a permis, dans un premier temps, de soutenir la thèse d'une relation entre l'exposition à un événement stressogène, un phénomène émotionnel et une altération du fonctionnement immunitaire. Alors que les premières études réalisées en PNI proposaient initialement une immunosuppression conditionnelle à la prévalence d'un état de stress-émotion intense et prolongé, les études réalisées en laboratoire confirment la notion d'intensité et infirment la notion de durée, justifiant ainsi la recherche liant l'émotion et l'immunité. L'association entre la réactivité immunitaire et l'activation du SNS appuie d'autant plus la condition d'intensité: les sujets hautement réactifs au niveau cardiovasculaire manifestent également une réactivité immunitaire supérieure. La recherche en laboratoire sur l'émotion et l'immunité permet de préciser que l'amplitude de la réactivité cardiovasculaire est plus importante que la valence positive ou négative de l'émotion. Par ailleurs, tout comme la recherche en milieu naturel, la recherche en laboratoire fait ressortir d'importantes différences individuelles dans les profils de réactivité immunitaire. Pour expliciter ces différences, l'intégration de ces travaux fait ressortir, dans un second temps, la nécessité d'inclure des variables médiatrices. Enfin la présentation de deux études confirme la contribution significative de l'évaluation cognitive dans 
l'explication des différences individuelles observées dans les profils de réactivité immunitaire. Par ailleurs, l'intégration de la recherche sur le stress et l'émotion permet de définir le stress psychologique selon une perspective globale (détresse) et spécifique (émotion). De la même façon, cette intégration encourage les efforts de recherche sur la définition opérationnelle d'un seul construit d'évaluation cognitive favorisant une utilisation globale ou spécifique. La nécessité de circonscrire le construit de stress se justifie par le besoin fréquent de restriction du nombre de variables dans certains protocoles de recherche. La possibilité d'élargir ce construit global par l'évaluation spécifique constitue assurément un ajout lorsque la description des profils de réaction nécessite plus de précision. L'ensemble de ce travail appuie la proposition de fusionner les deux domaines de recherche et encourage fortement l'inclusion d'un volet immunologique dans les études sur l'émotion.

\section{RÉFÉRENCES}

Ader, R. (1981). Psychoneuroimmunology. New York: Academic Press.

Ader, R., Felten, D.L., \& Cohen, N. (1991) Psychoneuroimmunology. (2nd ed.) New York: Academic Press.

Antoni, M. H. (1987). neuroendocrine influences in psychoimmunoology and neoplasia. Psychology anfd Health, 1, 3-24.

Arnetz, B.B., Brenner, S.O., Levi, L., Hjelm,R., Petterson, I.L., Wasserman, J., Petrini, B., Eneroth, P., Kallner, A., Kvetnansky, R., \& Vigas, M. (199I). Neuroendocrine and immunologic effects of unemployment and job insecurity. Psychotherapy Psychosomatic, 55, 76-80.

Arnetz, B.B., Wasserman, J., Petrini,B., Brenner, S.O., Levi,L., Eneroth, P., Salovaara, H., Hjelm, R., Salovaara, L., Theorell, T., \& Petterson, I.L. (1987). Immune function in unemployed woman. Psychosomatic Medicine, 49, 3-13.

Arnold, M. B. (1960). Emotion and Personality: Vol. I. Psychological aspects. N.Y.: Columbia University Presss.

Asterita, M. F. (1985). The physiology of stress. New York : Human Sciences Press.

Averill, J. R. (1973). Personal control over aversive stimuli and its relationship to stress. Psychological Bulletin, 80, 286-303.

Bachen, E.A., Manuck , S.B., Marsland, A.L.,Cohen, S., Malkoff,S.B., Muldoon, M.F., \& Rabin, B.S. (1992). Lymphocyte subset and cellular immune responses to a brief experimental stressor. Psychosomatic Medicine, 54, 673-679.

Bartrop, R.W., Lazarus, L., Luckhurst, E., \& Kiloh, L.G. (1977). Depressed lymphocytes function after bereavement. Lancet, I, 834-836.

Baum, A., Grunberg,N.E., \& Singer, J.E. (1992). Biochemical measurements in the study of emotion. Psychological Science, 3, 56-59. 
Blalock, J.E., \& Smith E.M. (1985). A complete regulatory loop between the immune system and neuroendocrine systems. Federation Proceedings, 44, 108-III.

Brown, G.W., \& Harris, T. (1978). Social Origins of Depression. New-York: Free Press.

Cacioppo, J.T. (1994). Social neuroscience: Autonomic, neuroendocrine, and immune responses to stress. Psychophysiology, 31, 113-128.

Cannon, W. (1931). Studies on the conditions of activity in the endocrine organs. American Journal of Physiology, 98, 447-452.

Coe, C., Rosenberg, L., \& Levine, V. (1988). Prolonged effect of psychological disturbance on the squirrel monkey. Brain, Behavior and Immunity, 2, 151-160.

Cohen, F. (1985). Stress and bodily illness. Dans A. Monat \& R.S. Lazarus (Eds.), Stress and coping (pp.40-54). N.Y. : Columbia Press University.

Dantzer, R., \& Kelley, K.W. (1989). Stress and immunity: integrated view of relationships between brain \& immune system. Life-Sciences, 44, 1995-2008.

Darko, D. F., Wilson, N.W., Gillin, J. C., \& Golshan, S. (|99|). A critical appraisal of mitogen-induced lymphocytes proliferation in depressed patients. American Journal of Psychiatry, 148, 337-344.

Daruna, J. H., \& Morgan, J. E. (1990). Psychosocial effects on immune function: neuroendocrine pathways. Psychosomatics, 31, 4-12.

Delongis, A., Coyne, J. C., Dakof, G., Folkman, S., \& Lazarus, R. S. (1982). Relationship of daily hassles, uplifts, and major life events to health status. Health Psychology, 1, I 19$-136$.

Ekman, P. (1984). Expression and the nature of emotion. Dans K.S. Scherer, \& P.Ekman (EDS) Approaches to emotion (319-343) Hillsdale, NJ:Erlbaum.

Felten, D. (199|). Neurotransmetter signalling of cells of the immune system: Important progress, major gaps. Brain, Behavior and Immunity, 5, 2-8.

Fillion, L. (1995). Cognitive appraisal, HIV status and immune reactivity. Dans L. Bessette (Ed.), Le deuil comme processus de guérison (pp. 126-128), Beauport: Publication $\mathrm{MNH}$.

Fillion, L. (1993). Évaluation cognitive, état de stress et immunité lors d'un diagnostic de tumeur au sein. Thèse de doctorat non-publiée. Québec: Université Laval.

Fillion, L., Belles-Isles, M., Lemyre, L., \& Roy, R. (1994). Reliability of lymphocyte proliferation assays. Stress Medicine, 10 ( 1 ).

Fillion, L., Kirouac, G., Lemyre, L., \& Mandeville, R. (1994). Stresseur et immunité.: une recension en psychoneuroimmunologie. Psychologie Canadienne, 35, 405-426.

Fillion, L., Lemyre, L., Mandeville, R., \& Piché, R. (1996). Facteurs cognitifs lors de l'adaptation psycho-immunologique au diagnostic de cancer du sein: implication clinique. Journal de Thérapie Comportementale et Cognitive, 6, 43-5I.

Fillion, L., Lemyre, L., Piché, R., \& Mandeville, R. (1996). Cognitive appraisal, stress state and cellular immunity before and after breast tumor diagnosis. International Journal of Rehabilitation and Health, 2, 169-187.

Fillion, L., Lemyre, L., Mandeville, R., Piché, R. Poisson, R., Fournelle-Lebuis, F. \& Falardeau, M. (1993). Cellular Immunity and psychological stress before and after diagnostic surgery of breast cancer. Clinical and Investigative Medicine, 16 (3).

Fillion, L., Tessier, R., Tawadros, E., \& Mouton, C. (1989). Stress et immunité: étude de validité d'une mesure de stress psychologique (MSP). Psychologie Canadienne, 30, 30-38.

Flannery, R. B. (1986). Personal control as a moderator variable of life stress: preliminary inquiry. Psychological Reports, 58, 200-202. 
Folkman, S. (1984). Personal control and stress and coping process: a theoretical analysis. Journal of Personality and Social Psychology, 46, 839-852.

Forsythe, C.J., \& Compas, B.E. (1987). Interaction of cognitive appraisals of stressful events and coping: Testing the goodness-of-fit hypothesis. Cognitive Therapy and Research, I I, 473-485.

Folkow, B. (1993). Psysiological organization of neurohormonal responses to psychosocial stimuli: implications for health and disease. Annals of Behavioral Medicine, 15, 236-244.

Futterman, A.D., Kemeny,M.E., Shapiro, D., \& Fahey, J.L. (1994). Immunological and physiological changes associated with induced positive and negative mood. Psychosomatic medicine, 56 : 499-511.

Frankenhaeuser, M. (1980). Psychoneuroendocrine approaches to the study of stressful person-environment transactions. Dans H. Selye (Ed)(pp.46-70). Selye's guide to stress research. N.Y.: Van. Nortrand Reinhold Company.

Friedman, E. S., Clark, D. B., Gershon, S. (1992). Stress, anxiety and depression : Review of Biological, diagnostic, and nosologic issues. Journal of Anxiety Disorders, 6, 337-363.

Glaser, R., Kennedy, S., Lafuse, W. P., Bonneau, R. H., Speicher, C., Hilhouse, J., \& Kiecolt-Glaser, J. K. (1990). Psychological stress induced modulation of interleukin 2 receptor gene expression and interleukin 2 production in peripheral blood leukocytes. Archives of General Psychiatry, 47, 707-719.

Glaser, R., Pearson, G.R., Bonneau, R.H., Esterling, B.A., Atkinson, C., \& Kiecolt-Glaser, J. (1993). Stress and the memory T-cell response to the Epstein-Barr virus in healthy medical students. Health Psychology, 12, 435-442.

Glaser, R., Rice, J., Speicher, C. E., Stout, J., \& Kiecolt-Glaser, J. K. (1986). Stress depresses interferon production by leukocytes concomitant with a decrease in N.K.C.A. Behavioral Neuroscience. 100, (5), 675-678.

Halvorsen, R., \& Vassend, O. (1987). Effects of examination stress on some cellular immunity function. Journal of Psychosomatic Research. 31, 693-701.

Heisel, J.S., Locke, S.E., Kraus, L.J., \& Williams, R.M. (1986). Natural Killer cell activity (NKCA) and MMPI scores of a cohort of college students. American Journal of Psychiatry, 143, 1382 $-1384$.

Herbert, T.B., \& Cohen, S. (1993). Stress and immunity in humans : A meta-analytic review. Psychosomatic Medicine, 55, 364-379.

Holm, J.E., Holroyd, K. A., Hursey, K. G., \& Penzien, D.B. (1986). The role of stress in Recurrent Tension Headache. Headache, 26, 160-167.

Holmes, T. H., \& Rahe, R. H. (1967). The social readjustment rating scale. Journal of Psychosomatic Research, 11, 213-218.

Horowitz, M.J., Wilner, N., \& Alvarez, W. (1979). Impact of event scale: A measure of subjective stress. Psychosomatic Medicine, 4I, 209-218.

Hurwitz, B.E., Nelesen, R.A., Saab, P.G., Nagel, J.H., Spitzer, S., Gellman, M., McCabe, P., Phillips, D.J., \& Schneiderman, N. (1993). Differential patterns of dynamic cardiovascular regulation as a function of task. Biological Psychology, 36, 75-95.

Irwin, M., Daniels, M., Risch, S. C., Bloom, E., \& Weinwe, H. (1988). Plasma cortisol and naturel killer cell activity (NKCA) during bereavement. Biological Psychiatry, 24, 173-178.

Irwin, M., Daniels, M., \& Weiner, H. (1987). Immune and neuroendocrine change during bereavement. Psychiatric Clinics North America, 10, 449-465.

Jemmott , J. B., \& Locke, S.E. (1984). Psychosocial factors, immunologic mediation and human susceptibility to infectious disease: How much do we know? Psychological Bulletin, 95, 78-108. 
Kedem, P., Bartoov, B., Mikulincer, M., \& Shkolnik, T. (1992). Psychoneuroimmunology and male infertility : a possible link between stress, coping and male immunological infertility. Psychology and Health, 6, 159-173.

Kiecolt-Glaser, J. K., \& Glaser, R. (1992). J. of Consulting and Clinical Psychology, 60, I-7.

Kiecolt-Glaser, J. K. (1988). Methodological issues in behavioral immunology research with humans. Brain, Behavior \& Immunity, 2, 67-78.

Kiecolt-Glaser, J. K., Galser, R., Strain, E., Stout, J., Tarr, K. L., Holliday, J. E., \& Speicher, C.E. (1986). Modulation of cellular immunity in medical students. Journal of Behavioral Medicine, 9, 5-21.

Kiecolt-Glaser, J. K., \& Glaser, R. (1988). Psychological influences on immunity. American Psychologist, 43, 892-898.

Kiecolt-Glaser, J.K., Glaser, R., Shuttleworth, E.C., Dyer, C.S., Ogrocki, P. \& Speicher, C.E. (1987b). Chronic stress and immunity in family caregivers of Alzheimer's disease victims. Psychosomatic Medicine. 49, (5), 523-535.

Knapp, P.H., Levy, E. M., Giorgi, R. G., Black, P. H., Fox, B. H., \& Heeren, T. C. (1992). Short-term immunological effects of induced emotion. Psychosomatic Medicine, 54, I33-148

Kronfol, Z., \& House, J.D. (1989). Lymphocyte mitogenis, immunoglobulin and complement levels in depressed patiens and normal controls. Acta Psychiatrica Scandinavia, 80, 142-147.

Lazarus, R.S. (1993). From psychological stresss to emotions: a history of changing outlooks. Annual Review of Psychology, 44, 1-21.

Lazarus, R. S. (199|). Theory based stress measurement. Psychological Inquiry, I, 4I-5I.

Lazarus, R. S. (1966). Psychological stress and the coping process. N.Y.: Mc-Graw Hill.

Lazarus, R. \& Folkman, S. (1984). Stress, appraisal and coping. N.Y.: Springer Pub. Company.

Lazarus, R. S., \& Launier, R. (1978). Stress-related transactions between person and environment.. In L.A. Pervin et M. Lewis (Eds). Interactional Psychology. NY. : Plenum Press.

Lemyre, L., \& Tessier, R. (in preparation). Stressor appraisal rating scales across methodologies: Perceived impact, mastery and uncertainty.

Lemyre, L. (1986). Stress et appréhension cognitive. Thèse non publiée. Univ. Laval, Québec.

Livnat, S., Felten, S.,Y., Bellinger, D. L., \& Felten, D. (1985). Involvement of peripherical and central catecholamine systems in neural-immune interactions. J.of Neuroimmunology, 10 , 5-30.

Manuck, S. B., Cohen , S., Rabin, B. S., Muldoon, M. F. \& Bachen, E. A. (1991). Individual differences in cellular immune response to stress. Psychological Science, 2, I| I-1 I5.

Mason, J.W. (1975). Emotion as reflected in patterns of endocrine integration. In L. Levi (Ed.), Emotions: Their parameters and measurement (pp. 143-181). N.Y. : Raven Press.

McNaughton, M. E., Smith, L.W., \& Grant, I. (1990). Stress, social support, coping resources, and immune status in elderly women. Journal of Nervous and Mental Disease, 178, 460-461 .

Miller, T. (1988). Advances in understanding the impact of stressful life events on health. Hospital and Community Psychiatry, 39, 6, 615-622.

Minter, R. E., \& Patterson, C. (1978). Life events and illness onset: a review. Psychosomatics, 19, 334-339.

Monroe, S.M., \& Kelley, J.M. (1995). Measurement of stress appraisal. Dans S. Cohen, R.C. kessler, \& L.U.Gordon (Eds), (122-147). Measuring Stress. N.Y.: Oxford Univesity Press.

Moos, R.H., \& Schaefer, S.C. (1993). Coping ressources and processes: Current concepts and measures. Dans L. Goldberger \& S. Breznitz (Eds.) (234-257). Handbook of stress (2nd edition). N.Y.: The Free Press. 
Naliboff, B. D., Benton, D., Solomon, G. F., Morley, J. E., Fahey, J. L., Bloom, E.T., Makidodan, T., \& Gilmore, S. L. (199I). Immunological changes in young and old adults during brief laboratory stress. Psychosomatic Medicine, 53, 121-132.

O'Leary, A. (1990). Stress, emotion and human immune function. Psychological Bulletin, 108, 363-382.

Parkes, K. R. (1986). Coping in stressful episodes: the role of individual differences, environmental factors and situational characteristics. Journal of Personnality and Social Psychology, 51, 1277-1292.

Paterson, R. J., \& Neufeld, R.W.J. (1987). Clear danger: Situational determinants of the appraisal of threat. Psychological Bulletin, 101, 404-416.

Paykel, E. S. (1987). Methodology of life events research. Advances in Psychosomatic Medicine, 17. 13-29.

Pelletier, R., \& Herzing, D. L. (1989). Psychoneuroimmunolgy: Toward a mind body model: A critical review. Advances, 5, 27-56.

Roitt, I. (1992). Essential Immunology (7ème Ed.) London: Blackwell.

Roseman, I. J. (1984). Cognitive determinants of emotions : A structural theory. Dans P. Shaver (Ed.) Review of Personality and social psychology (1 I-36). Bevely Hills, CA: Sage.

Roseman, I. J., Spindel, M. S., \& Jose, P. E. (1990). Appraisal of emotion eliciting events: Testing a theory of discrete emotions. J. of Personality and Social Psychology, 59, 899-925.

Sarason, I. G., Johnson, J. H., \& Siegel, J. M. (1978). Assessing the impact of life changes: Development of the Life Experience Survey. J. Consulting and Clinical Psychology, 46, 932-946.

Scherer, K. R. (1988). Criteria for emotion-antecedent appraisal: A review. Dans V. Halminton, G.H. Bower, \& N.H.Frijda (Eds.). Cognitive perpectives on emotion and motivation (pp. 89 -126). Norwell, MA: Kluwer Academic.

Scherer, K. R. (1990). Stress et coping: nouvelles approches. Cahiers Psychiatriques Genevois, $9,147-154$.

Schleifer, S. J., Keller, S. E., Bond, R. N., Cohen, J., \& Stein, M. (1989). Major depressive disorder and immunity. Archives of General Psychiatry, 46, 81-87.

Schleifer, S. J., Keller, S. E., Camerino, M., Thornton, J. C., \& Stein, M. (1983). Suppression of lymphocyte stimulation following bereavement. JAMA, 250, 374-377.

Schneiderman, N., \& McCabe, P. (1989). Psychophysiologic strategies in laboratory research. In N. Schneiderman, S.M. Weiss, \& P.G. Kaufman (Eds.), Handbook of research methods in cardiovascular behavioral medicine (pp349-364). N.Y.: Plenum.

Selye, H. (1950). The physiology and pathology of exposure to stress. Montréal: Acta.

Sgoutas-Emch, S.A., Cacioppo, J.T., Uchino, B.N., Malarkey, W., Pearl, D., Kiecolt-Glaser, J., \& Glaser, R. (1994). The effects of an acute psychological stressor on cardiovascular endocrine, and cellular immune response: A prospective study of individuals high and low in heart rate reactivity. Psychophysiology, 31, 264-27l.

Sinha, R., Lovallo, W.R., \& Parsons, O.A. (1992). Cardiovascular differentiation of emotions. Psychosomatic Medicine, 54, 422-435.

Smith, C.A. (1989). Dimensions of Appraisal and physiological response in emotion. Journal of Personality and Social Psychology, 56, 339-353.

Smith, C.A., \& Ellsworth, P. C. (1985). Patterns of Cognitive Appraisal in emotion. Journal of Personality and Social Psychology, 48, 813-838.

Smith,C.A., \& Lazarus, R.S. (1993). Appraisal components, core relational themes, and the emotions. Cognition \& Emotion, 7, 233-269. 
Stone, A.A., Valdimarsdottir, H.B., Katkin, E.S., Burns, J., \& Cox, D.S. (1993). Effects of mental stressor on mitogen-induced lymphocyte responses in the laboratory. Psychology and Health, 8, 269-284.

Syvalahti, E. (1987). Endocrine and immune adaptation in stress. Annals of Clinical Research, 19, 70-77.

Thompson, S. C. (198I). Will it hurt less if I can control it ? A complex answer to a simple question. Psychological Bulletin, 90, 89-101.

Udelman, D. L., \& Udelman, H. D. (199|). Affects, neurotransmitters and immuno-competence. Stress Medicine, 7, 159- 162.

Ursin, H. (1982). The search for stress markers. Scandinavian J.of Psychology, Supp, I65-169.

Vinokur, A., \& Selzer, M. L. (1975). Desirable versus undesirable life events: their relationship to stress and mental distress. Journal of Personality and Social Psychology. 32, 329-337.

Vingerhoests, A. J. J. M., \& Assies, J. (199|). Psychoneuroendocrinology of stress and emotions: Issues for future research. Psychotherapy Psychosomatic , 55, 69-75.

Watson, D., \& Pennebaker, J.W. (1989). Health complaints, stress, and distress: Exploring the central role of negative affectivity. Psychological Review, 96, 234-254.

Walbott, H. G., \& Scherer, K. R. ( 199 |). Stress specificities: Differential effects of copyng style, gender, and type of stressor on autonomic arousal, facial expression and subjective feeling. Journal of Personality and Social Psychology, 61, 147-156.

Weisse, C. S. (1992). Depression and immunocompetence: A review of the literature. Psychological Bulletin, 111, 475-489.

Weisse, C. S., Pato, C. N., McAllister, C.G., Littman, R., Breier, A., Paul, S.M., \& Baum, A. (1990). Differential effects of controllable and uncontrollable acute stress on lymphocyte proliferation and leukocyte percentages in humans. Brain, Behavior \& Immunity, 4, 339-35I.

Workman, E. A ., \& LaVia, M.F. (1987). T-lymphocyte polyclonal proliferation and stress response style. Psychological Reports, 60, $1121-1122$.

Zautra, A. J., Okun, M. A., Robinson, S. E., Lee, D., Roth, S.H., \& Emmanual, J. (1988). Life stress and lymphocites alterations among patiens with rheumatoid arthritis. Health Psychology, $8,1-14$.

Zakowski, S.G.,Cohen L., hall, M.H., Wollman, K., \& Baum, A. (1992). Differential effects of active and passive laboratory stressors on immune function in healthy man. International Journal of behavioral Medicine, I, 163-184.

Zakowski, S.G., McAllister, C. G., Deal, M. , \& Baum, A. (1992). Stress, Reactivity, and Immune Function in Healthy Men. Health Psychology, 11, 223-232.

Zegans, L.S. (1982). Stress and the development of somatic disorders. In L. Goldberg \& S. Breznitz (Eds), Handbook of stress ( Pp |34- 152). N.Y. : Free Press. 


\section{Ramon Bayés}

Universitat Autònoma de Barcelona

\section{PERCEPTION DES SYMPTÔMES ET RÉACTIONS ÉMOTIONNELLES LORS DES MALADIES MORTELLES}

L'émotion, la peur, la souffrance et la douleur sont des mots familiers aussi bien dans le langage de tous les jours que dans la pratique clinique et la recherche dans le domaine des soins palliatifs lors des maladies mortelles.

Les émotions négatives, telle la peur, caractérisent la plupart des mammifères et elles ont évolué pour favoriser l'adaptation et, en général, améliorer la survie des espèces (Darwin, 1872; Izard, 1993; MacLean, 1990; Myers, 1992). La peur est souvent une réaction adaptative. La peur prépare nos corps pour fuir du péril; la peur d'une lésion nous protège du dommage; la peur des représailles contrôle notre agressivité. On croit, en conséquence, que les émotions négatives associées à la douleur et aux pertes sont investies d'une mission biologique et, bien que n'étant apparemment d'aucune utilité pour les malades en fin de vie, elles peuvent, en beaucoup d'autres circonstances, agir comme une ressource efficace pour la personne.

L'idée de souffrance, d'autre part, bien qu'elle ait une signification émotionnelle très claire et de grande importance dans la vie humaine (Cassell, 1982), est très difficile à définir avec précision. Pour Chapman et Gravin (1994) le sentiment d'impuissance est un facteur déterminant dans la souffrance et, après une analyse de la littérature, ces auteurs nous indiquent qu'une définition adéquate de la souffrance doit posséder les éléments suivants: $a$ ) un sentiment de menace à l'intégrité du soi (physique et/ou psychosocial); b) un sentiment d'impuissance et de privation; et c) un caractère émotionnel durable et désagréable. En synthèse, ces auteurs définissent la souffrance comme «un état affectif, cognitif et négatif complexe caractérisé par la sensation d'être menacé dans son intégrité, par le sentiment d'impuissance ressenti face à cette menace et par l'épuisement des ressources 
personnelles et psychosociales qui permettent de l'affronter» (pp. 38). Ses racines se trouvent, probablement, dans la capacité des êtres humains d'anticiper les conséquences des événements (Craig, 1984), laquelle apparaît très tôt puisque Levy (1960) a déjà observé que les enfants, à partir de 6 mois, montrent des réactions de peur sévères devant les médecins et infirmières s'ils ont une histoire de piqûres et de vaccins.

L'International Association for the Study of Pain (IASP), pour sa part, définit la douleur comme étant «...une expérience sensorielle et émotionnelle désagréable, associée à des lésions tissulaires réelles ou probables, ou décrite en fonction de tels dommages» (Cfr. Merskey, 1979). Comme soulignent Chapman et Gavrin (1994), la définition de I'IASP suggère au moins deux types de processus: a) sensoriel, qui livre au cerveau des informations de type spatial, temporel et qualitatif; et b) émotionnel, qui colore de façon négative cette perception sensorielle et qui s'apparente à une menace. En conséquence, l'expérience de la douleur est, partiellement, une expérience émotionnelle et les aspects émotionnels de la douleur sont apparemment discriminables de ses qualités sensorielles (Craig, 1984).

Dans une recherche déjà classique, Beecher (1956, 1959) a montré dramatiquement l'importance des facteurs psychologiques dans la modulation de la douleur en comparant les données précédentes de 150 soldats des États Unis qui ont été blessés sur la plage d'Anzio pendant la deuxième guerre mondiale, avec celles de 150 patients civils du même âge habitant les États Unis et qui ont été soumis à une intervention chirurgicale avec une affection similaire de leur organisme. Tandis que seulement 32\% des premiers ont manifesté qu'ils avaient besoin d'analgésiques pour combattre la douleur, $83 \%$ des civils réclamaient ce type de médicaments. Pour Beecher, «il n'existe pas une relation simple entre la blessure per se et la douleur. La douleur se trouve déterminée, en bonne partie, par d'autres facteurs et, dans ce cas-là, la signification des 212 blessures pour les affectés était de la plus grande importance». Pour les soldats, les blessures sont associées au retour au foyer et à la fin des combats; pour les civils, les blessures n'ont que des connotations négatives et peuvent être considérées comme une menace contre l'intégrité physique. Comme le souligne Craig (1984), «la perception de la douleur peut être atténuée ou accentuée dans les processus émotionnels» (pp. 155). La peur, l'anxiété et la dépression peuvent amplifier la douleur et les thérapeutiques pharmacologiques et psychologiques qui diminuent l'anxiété et la tension réduisent souvent la douleur clinique. 
Baines (1990), conseillère médicale du St. Christopher Hospice de Londres, en référence à la douleur des malades en fin de vie nous signale: «C'est probablement assez exceptionnel que la douleur soit produite par des facteurs non physiques. Néanmoins, les problèmes émotionnels et sociaux rendent souvent plus violente la douleur et, de fait, il s'établit un cercle vicieux dans lequel la douleur physique conduit vers l'anxiété et la dépression, et ces dernières, à leur tour, diminuent le seuil des sensations douloureuses»). Mount (1984), l'introducteur des unités de soins palliatifs au Canada, écrit que «la douleur du cancer a son origine dans une pathologie physique nuisible, mais elle est toujours modifiée par l'esprity (pp. 460). Cassell (1982) nous montre que la signification perçue de la douleur influe dans la quantité d'analgésique qui sera nécessaire pour la contrôler. Et Stedeford (1984), finalement, nous signale que quand les symptômes comme la douleur n'apportent aucun soulagement avec les mesures thérapeutiques qui sont habituellement effectives, le médecin doit soupçonner que ce sont des problèmes psychologiques qui les rendent plus violents ou encore qui les provoquent. Clairement, les processus psychologiques ne peuvent être ignorés même dans les cas où il existe une base biologique solide pour la douleur.

La douleur et la souffrance se partagent la dimension négative de l'émotion, mais la relation entre elles est imprécise. On peut bien éprouver de la douleur et ne pas souffrir, c'est à dire, bien que la douleur puisse être désagréable, elle ne cause pas de souffrance si la personne la perçoit comme non menaçante - par exemple, dans l'accouchement sans problèmes ou dans le rapatriement des soldats blessés de Beecher $(1956,1959)$ - et, à l'inverse, on peut aussi souffrir et ne pas éprouver de la douleur - comme dans l'attente du diagnostic d'une biopsie ou d'une preuve sérologique au VIH, occasions dans lesquelles la personne éprouve, dans l'impuissance, un sentiment de menace. Donc, il ne suffit pas d'éprouver des douleurs pour souffrir et on peut souffrir sans éprouver une douleur physique. Cependant, il y a de fortes chances que la douleur - surtout en cas de maladie grave - provoque de la souffrance. Ce n'est pas que toute souffrance ait son origine dans la douleur - comme l'ont écrit Loeser et Melzack (1999) mais dans notre culture médicalisée, nous exprimons la souffrance dans le langage de la douleur.

Ce que nous disons de la douleur nous pouvons l'étendre, chez un grand malade, à d'autres symptômes physiques (Bruera, 1993) perçus par lui comme menaçants, comme, par exemple, la dyapnée, l'insomnie, la faiblesse, 
la nausée, la diarrhée ou le vomissement. Et, de façon pareille à la douleur, on peut bien éprouver de la dyapnée et de la faiblesse, par exemple, après une course athlétique, ou de l'insomnie si on remporte le grand prix du loto, et ne pas souffrir pour eux.

Non seulement des appréhensions d'ordre physique assaillent le malade en fin de vie - peur de la douleur, de perdre son image corporelle, son autonomie, peur des complications qui peuvent survenir, peur de l'agonie mais bien d'autres peurs sont souvent présentes: peur d'être dépendant des autres, de perdre le contrôle de la situation, peur de ne pas avoir le temps d'accomplir des souhaits intimes, peur de la séparation, de l'avenir pour ceux qui resteront, de l'incertitude que «l'après mort» pose aux croyants.

Il faut aussi penser que ces peurs agissent sur une personne dont l'esprit peut être calme, mais qui peut être aussi dépressif ou anxieux.

Nous pouvons représenter ces idées précédentes de la façon que montre le modèle de la Figure I (Bayés, Arranz, Barbero y Barreto, 1996).

Perception

sensorielle

désagréable

(douleur,

dyapnée, etc.)

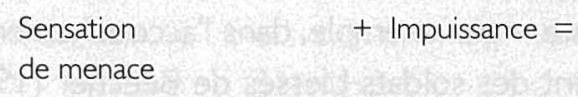

\section{Souffrance}

État d'esprit

Perception

psychosociale

désagréable

(séparation,

perte de contrôle,

etc.)

(Bayés, Arranz, Barbero et Barreto, 1996)

Figure I - Relation entre perception sensorielle et/ou psychosociale désagréable, et souffrance 
À mesure qu'une maladie grave - le cancer, le SIDA, etc. - avance, les réactions psychologiques opèrent une intégration complexe entre la mémoire des expériences passées, la perception des menaces futures et les ressources disponibles (Razavi et Delvaux, 1994). La phase terminale est souvent riche d'événements et d'échanges vécus intensément. Des pertes de rôles et de fonctions peuvent constituer des crises existentielles.

En résumé, nous avons peur:

a) de ne pouvoir jamais récupérer ce que nous avons déjà perdu.

b) de perdre tout ce que nous avons encore.

c) de ne pouvoir avoir jamais ce qui nous manque et que nous souhaitons intensivement d'avoir.

Les symptômes physiques, les réactions d'autrui et les situations perçues par le malade comme menaçantes sont les signes qui déclenchent la peur et la souffrance. Alors, il sera très important, si nous voulons aider ce malade, de connaître quelles sont, personnellement, pour lui et dans chaque moment précis, les choses qui l'inquiètent et qui, peut être, rendent sa vie un enfer.

En effet, d'une part, il y a des peurs, on peut dire biologiques, qui ont besoin, pour faire leur apparition dans des individus d'une espèce précise, d'un apprentissage faible ou presque nul, comme la peur des serpents et des araignées, et surtout, la peur de la mort. Lorsque Donald Hebb (1980) a montré pour première fois un modèle en argile de tête de chimpanzé à un groupe d'animaux, ils ont été pris de panique, et leur réaction a été semblable à celle des personnes qui voient le corps mort ou écartelé d'un être humain. Mais, d'autre part, le long de notre histoire personnelle dans une culture précise, moyennant de complexes associations, des observations et des interactions, nous pouvons apprendre à craindre presque quoi que ce soit et à avoir peur de choses ou d'événements différents de ceux qui font peur à d'autres. Le seul moyen de connaître quels symptômes, comportements et situations ont atteint la condition de menaçants pour une personne est de chercher à connaître quelles sont les choses où les situations qui l'inquiètent.

Pour la plupart des personnes concernées, la mort induit un vécu de rupture et de séparation définitives. Toutes ces réactions peuvent mener à une adaptation ou à un échec (Lipowski, 197I; Lazarus, 1982) et souvent peuvent donner lieu à une détresse émotionnelle qui se traduit en termes d'anxiété et de dépression. 
Si nous partons de la définition de Chapman et Gravin (1994), il est évident qu'on peut aborder la souffrance du malade de différentes façons, complémentaires et non alternatives:

a) Avec le contrôle de la douleur et d'autres symptômes perçus par le patient comme menaçants - une douleur sévère peut suffire pour causer et maintenir la souffrance - et pour que les soignants puissent agir efficacement, il faudra se demander souvent quels sont ces symptômes biologiques, psychologiques ou sociaux - perçus comme menaçants. De fait, il faut construire et soutenir dès le début une relation de confiance entre les soignants, le patient et sa famille. Quand la maladie est terminale, l'assurance que les soignants ont l'intention de contrôler les symptômes perçus comme menaçants contribue pour beaucoup à prévenir la souffrance. II faut se souvenir, par exemple, que toute difficulté respiratoire peut s'accompagner d'anxiété chez le malade et ses familiers (Mouren-Mathieu, 1987). Elle trouble le sommeil du malade autant pour des raisons physiques que par crainte de ne pas se réveiller. Pour répondre efficacement à cette angoisse, il faut connaître les peurs du malade: le manque d'air?, l'ignorance de la cause de ses difficultés?, la mort?, des préoccupations familiales?. Pour Mouren-Mathieu (1987), dans cette situation, il faut rassurer le malade en l'informant sur l'étiologie de sa dyapnée en lui posant des questions telles comme «Avez-vous peur de quelque chose?»». Personnellement, nous préférons la question «Avez-vous du souci pour quelque chose?».

b) Avec la minimalisation de l'impuissance qu'il ressent, en l'aidant à réajuster ses buts sur une base quotidienne. II faut encourager le malade à accepter ses limitations et se poser des objectifs réalistes: lui faire accepter progressivement une canne, une chaise roulante qui lui conserveront une autonomie relative: pouvoir encore sortir ou se mettre à table.

c) En lui maximisant ses propres ressources et en lui en fournissant de nouvelles - comme, par exemple, la relaxation - pour contrôler le mieux possible ses émotions et ses activités cognitives, alors que ses capacités physiques déclinent abruptement. En aidant le patient à acquérir le sentiment de «maîtriser la morts, le soignant contribue à prévenir la souffrance du patient et de sa famille.

Quant au problème de l'information, il faut souligner, avec Gómez Sancho (1994), que «La vérité est l'antidote de la peur. Les événements terribles et connus sont préférables aux événements terribles mais inconnus» (pp. 287).

Pour accomplir le programme d'interventions précédent, il est nécessaire que nous puissions connaître: 
a) Quels symptômes physiques sont pour le malade une source de menace

b) Quels facteurs psychosociaux sont pour le malade une source de menace.

c) Quelle information possède la personne sur sa maladie, son traitement et sa situation.

d) De quels recours dispose le malade pour affronter la situation.

La présence imminente de la mort - sa propre mort ou celle d'une personne bien-aimée - est, à notre avis, une situation naturelle de choix pour l'étude des émotions plus intenses que peut éprouver l'homme. Vachon (1993) nous signale que les données correspondant à des échantillons représentatifs randomisés de sous populations du Canada nous montrent qu'entre le 61 et le 79 pour cent des patients des unités de soins palliatifs présentent une douleur psychologique (distress) sévère par rapport à 18 -34 pour cent chez le groupe de comparaison.

De plus, son intérêt comme sujet de recherche est, à notre avis, prioritaire pour les professionnels de la santé, étant donné que plus nous pourrons connaître et maîtriser la souffrance plus nous pourrons aider un grand nombre de personnes à parcourir, sans peur - ou avec la peur diminuée ou contrôlée - et avec dignité, le dernier chapitre de leur existence. La connaissance et le contrôle de la souffrance représentent pour nous tous, chercheurs et cliniciens, un formidable défi.

Et si nous voulons arriver au bout, si nous voulons construire des équipes multidisciplinaires de soins palliatifs efficaces (Bayés et Barreto, 1992), nous devons, avant tout, connaître quels sont, de façon évolutive vers la mort, les peurs, les soucis, les symptômes - biologiques, psychologiques, sociaux - perçus comme menaçants par les patients en phase terminale.

C'est dans ce but qu'il y a quelques années nous avons pris part à une recherche menée dans deux hôpitaux espagnols qui ont des services de soins palliatifs (Comas, Martínez, Barreto, Bayés, Roca, Pascual, Gómez-Batiste, y García Conde, 1993) et que, lorsque cette recherche a été finie, nous nous sommes engagés dans un projet multicentre avec le concours de quinze hôpitaux (Bayés, Barreto, Comas y Limonero, 1997).

Les données que je vous présente maintenant correspondent à 37। malades ( 314 de cancer et 57 de SIDA) en fin de vie avec un traitement thérapeutique exclusivement palliatif. 
Le même questionnaire - avec 33 symptômes issus de la littérature et des résultats d'une recherche précédente (Comas et al., 1993) a été administré à chaque malade jusqu'au moment de sa mort, de façon systématique, le plus grand nombre de fois qu'il a été possible, en accord avec le principe éthique de profit du malade et d'opportunité clinique. Le but principal de cette recherche prospective a été de connaître l'évolution de la perception des symptômes et des soucis à mesure que la mort approche (Cfr. Bayés, 1995).

II faut se souvenir qu'une caractéristique des malades atteints du cancer et du SIDA en fin de vie - en contraste avec les malades chroniques - est le changement fréquent de leurs symptômes et problèmes (Sanz, Gómez-Batiste, Gómez-Sancho et Núñez Olarte, 1993; Chochinov, Tataryn, Clinch y Dudgeon, 1999).

Dans le Tableau I vous pouvez trouver les 15 symptômes perçus par le plus grand nombre de malades (Bayés y Limonero, 1999)

TABLEAU I - Symptômes les plus perçus par les malades en fin de vie

\begin{tabular}{|c|c|c|}
\hline & Synptôme & $\begin{array}{c}\% \text { de malades qui } \\
\text { le perçoivent }\end{array}$ \\
\hline 1) & Faiblesse générale & 86 \\
\hline 2) & Perte de poids & 72 \\
\hline 3) & Somnolence diurne & 68 \\
\hline 4) & Tristesse & 65 \\
\hline & Anorexie & 65 \\
\hline 5) & Nervosité & 63 \\
\hline 6) & Douleur & 57 \\
\hline 7) & Constipation & 53 \\
\hline 8) & Troubles de sommeil & 52 \\
\hline 9) & Problèmes de vision & 44 \\
\hline 10) & Problèmes de respiration & 43 \\
\hline 11) & Troubles de langage & 41 \\
\hline 12) & Nausée ou vomissement & 39 \\
\hline 13) & Mal à la bouche & 38 \\
\hline 14) & Toux & 37 \\
\hline
\end{tabular}

(Bayés et Limonero, 1999) 
De ce Tableau, nous pouvons déjà déduire qu'il n'y a aucun symptôme qui soit perçu par tous les malades. II n'y a pas de symptômes universels. II n'y a pas de maladies, mais des malades.

Dans le Tableau 2, d'autre part, nous pouvons observer, à partir des symptômes qui sont perçus, quels sont ceux qui préoccupent le plus les malades.

TABLEAU 2 - Symptômes perçus qui inquiètent le plus les malades en fin de vie

\begin{tabular}{|c|c|c|}
\hline \multicolumn{2}{|r|}{ Symptôme } & $\begin{array}{l}\text { \% de malades qui s'en inquiètent } \\
\text { par rapport à ceux qui le perçoivent }\end{array}$ \\
\hline 1) & Faiblesse générale & 50 \\
\hline 2) & Anorexie & 45 \\
\hline 3) & Paralysie & 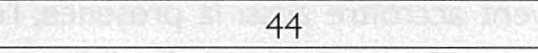 \\
\hline 4) & Douleur & 38 \\
\hline 5) & Perte de poids & 32 \\
\hline 6) & Problèmes de respiration & 30 \\
\hline & Constipation & 30 \\
\hline 7) & Nausée ou vomissement & 27 \\
\hline & Troubles de sommeil & 26 \\
\hline & Tristesse & 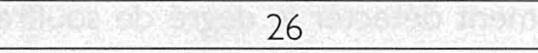 \\
\hline 9) & Aspect physique & 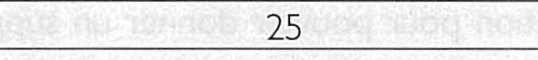 \\
\hline & Mal à la bouche & 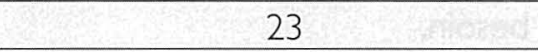 \\
\hline 11) & Solitude & 22 \\
\hline 12) & Somnolence diurne & 20 \\
\hline 13) & Difficultés de déglutition & 19 \\
\hline
\end{tabular}

(Bayés et Limonero, 1999)

De ces deux Tableaux, nous pouvons déduire:

a) Les symptômes perçus par le plus grand nombre de malades ne sont pas nécessairement ceux qui les inquiètent le plus (p.ex.: somnolence diurne, constipation et problèmes visuels). 
b) Quelques-uns des symptômes qui sont perçus par un nombre relativement petit de malades produisent un grand souci (p.ex.: paralysie, problèmes de respiration et mal à la bouche).

c) Quant aux symptômes physiques les plus inquiétants (faiblesse générale, anorexie, paralysie, douleur et problèmes de respiration), entre la moitié et les $2 / 3$ des malades qui les perçoivent ne les considèrent pas très menaçants, ce qui nous fait penser au schéma de la Figure 1.

d) Le degré de menace de chaque symptôme particulier dépendra de l'histoire et des caractéristiques personnelles du malade. Alors, la seule façon de connaître si un des symptômes perçus est pour lui cause de souffrance sera de le questionner, d'une façon éthique, sur le degré d'inquiétude que ce symptôme produit chez lui.

e) Quelques symptômes psychologiques, comme la tristesse (dépression) et le nervosité (anxiété), et d'autres symptômes psychosociaux, comme la solitude ou la dépendance, provoquent un degré de souci considérable et peuvent accroître aussi la présence, l'intensité ou la sensation de menace des symptômes physiques. Ils doivent, donc, si possible, être contrôlés.

De façon évolutive, nous avons observé, par exemple, que la tristesse augmente dans les 30 jours qui précédent le moment de la mort.

Dans le présent contexte, notre intention n'est pas de parler de mesures thérapeutiques particulières. Mais elle est, peut être, de nous questionner comment détecter le degré de souffrance de chaque malade dans chaque situation pour pouvoir donner un support prioritaire a ceux qui en ont le plus besoin.

Dans ce but, dans un contexte clinique, notre modèle de criblage (screening) est le thermomètre: II est facile à appliquer; on peut le faire servir autant de fois qu'on le désire; et il nous indique si les choses vont mieux, si elles vont plus mal ou si elles se maintiennent sans variation. Évidemment, comme la souffrance est subjective, il faudra questionner le malade et nos questions ne devront pas, pour des raisons éthiques, troubler et augmenter sa souffrance. Est-ce qu'il est possible d'avoir un thermomètre clinique de la souffrance humaine qui accomplisse ces conditions?

Nous croyons que la réponse est affirmative. Notre idée est issue d'un vieux texte de William James (1890) qui a plus de cent ans et dans lequel l'auteur nous parle des différences entre le temps chronologique et le temps subjectif. 
De fait, l'expérience subjective nous montre que, quand nous nous trouvons dans une situation agréable, le temps perçu nous semble plus court que le temps chronologique; mais, si la situation est désagréable, le temps s'allonge et peut se faire interminable. Nous croyons que questionner un malade sur sa perception subjective d'une période chronologique particulière, peut être un indicateur bon et simple du degré de bien-être ou de malaise que le malade éprouve dans la période considérée (Bayés, Limonero, Barreto y Comas, 1995). Évidemment, avec cette méthode, nous ne pouvons savoir si la cause d'un possible malaise est une profonde souffrance ou un simple ennui. Pour le dévoiler, il nous faudra continuer l'exploration en profondeur (Bayés, 2000). Mais nous nous trouvons dans un problème semblable avec le thermomètre clinique. II nous indique la fièvre mais non pas ses causes. Pour les connaître il nous faudra interroger le malade, l'explorer, lui demander des analyses.

Notre thermomètre émotionnel est un simple instrument de criblage. Mais il nous permet, d'une façon simple et éthique, de détecter quelques-uns des malades qui ont le plus besoin de notre aide.

Nous avons étudié avec cette méthode 655 situations différentes chez les 37 I malades en fin de vie de notre recherche. Nos résultats montrent que dans $83 \%$ des cas dans lesquels les malades nous indiquent qu'ils ont perçu le temps comme «Long» ou «Très long» ils qualifient leur état général comme «Médiocre», «Mauvais» ou "Très mauvais» et que dans le 76,8\% des cas où ils nous indiquent qu'ils ont expérimenté le temps comme "Court» ou «Très court» leur degré de bien-être a été perçu comme «Bon» ou «Très bon». Ces données sont statistiquement significatives $\left(X^{2}=129,7\right.$, d.I. 2, $\mathrm{P}<0.00$ I) (Bayés, Limonero, Barreto et Comas, 1997).

L'étude des émotions et des cognitions devant la mort est un sujet important et difficile. Ces émotions et cognitions constituent les éléments d'une situation que nous devrons tous vivre un jour. Mieux les connaître nous aidera, peut-être, à l'affronter avec moins de peur et de souffrance, et à aider d'autres êtres humains à faire de même.

\section{RÉFÉRENCES}

Baines, M. (1990). Tackling total pain. En C. Saunders (Ed.) Hospice and palliative care (pp. 26-38). Londres: Arnold.

Bayés, R. (1995). SIDA y psicología. Barcelona: Martínez Roca. 
Bayés, R. (2000). Una estrategia para la detección del sufrimiento en la práctica clínica. Revista de la Sociedad Española del Dolor, 7, 70-74.

Bayés, R., Arranz, P., Barbero, J. et Barreto, P. (1996). Propuesta de un modelo integral para una intervención terapéutica paliativa. Medicina Paliativa, 3, 114-121.

Bayés, R. et Barreto, P. (1992). Las unidades de cuidados paliativos como ejemplo de interdisciplinariedad. Clínica y Salud, 3 (1), I I-19.

Bayés, R., Barreto, P., Comas, M.D. et Limonero, J.T. (1997). Multicentric research on assessment of symptoms and worries perceived by terminally ill patients. Dans F. De Conno (Ed.) Proceedings of the IV Congress of the European Association of Palliative Care (pp. 579-582). Milan: European Association of Palliative Care.

Bayés, R. et Limonero, J.T. (1999). Prioridad en el tratamiento de los síntomas que padecen los enfermos oncológicos en situación terminal. Medicina Paliativa, 6, 19-21.

Bayés, R., Limonero, J.T., Barreto, P. et Comas, M.D (1995). Assessing suffering. The Lancet, 346, 1492.

Bayés, R., Limonero, J.T., Barreto, P. et Comas, M.D. (1997). A way to screen for suffering in palliative care. Journal of Palliative Care, 13 (2), 22-26.

Beecher, H.K. (1956). Relationship of significance of wound to the pain experience. Journal of The American Medical Association, 161, 1609-1613.

Beecher, H.K. (1959). Measurement of subjective responses: quantitative effects of drugs. Nueva York: Oxford University Press.

Bruera, E. (1993). Research in symtoms other than pain. Dans D. Doyle, G.W.C. Hanks et N. MacDonald (Eds.) Oxford texbook of palliative care (pp. 87-92). Oxford: Oxford University Press.

Cassell, E.J. (1982). The nature of suffering and the goals of Medicine. The New England Journal of, 306, 639-645.

Comas. M.D., Martínez, E, Barreto, M.P., Bayés, R., Roca, J., Pascual, A., Gómez-Batiste, X. et García Conde, J. (1993). Assessment of the perception of symptoms and worries in Spanish terminal patients (IV). Comunication presenté au 3er Congreso Latini Dies. Toulouse, Mai.

Craig, K.D. (1984). Emotional aspects of pain. Dans P.D. Wall et R. Melzack (Eds.) Texbook of pain (pp. 153- 161). Edinburg: Churchill Livingstone.

Chapman, C.R. et Gavrin, J. (1994). La souffrance et sa relation avec la douleur. Infokara, $n^{\circ} 34,24-43$.

Chochinov, H.M., Tataryn, D., Clinch, J.J. et Dudgeon, D. (1999). Will to life in the terminally ill. The Lancet, 354, 816-819.

Darwin, C. (1872). The expression of emotions in man and animals. London: John Murray.

222 Gómez Sancho, M. (Ed.) (1994). Cuidados paliativos e intervención psicosocial en enfermos terminales. Las Palmas de Gran Canaria: Instituto Canario de Estudios y Promoción Social y Sanitaria.

Hebb, D.O. (1980). Essay on mind. Hillsdale, N.J:: Erlbaum.

Izard, C. (1993). Four systems for emotion activation: Cognitive and noncognitive processes. Psychological Review, 100, 68-90.

James, W. (1890). Principles of psychology (Edition de 1980). New York: Holt

Lazarus, R.S. (1982). Stress and coping as factors in health and illness. Dans J. Cohen et al. (Eds.) Psychosocial aspects of cancer (pp. 163-190). New York: Raven.

Levy, D.M. (1960). The infant's earliest memory of inoculation. Journal of Genetic Psychology, $96,3-46$. 
Lipowski, Z. (1971). L'individu face à la maladie psychique. Revue de Médecine Psychosomatique et de Psychologie Médicale, 13 (3), 235-249.

Loeser, J.D. et Melzack, R. (1999). Pain: an overview. The Lancet, 353, 1607-1609.

MacLean, P.D. (1990). The triune brain in evolution: Role in paleocerebral functions. New York: Plenum.

Merskey, H. (1979). Pain terms: A list with definitions and a note on usage. Recommended by the International Association for the Study of Pain (IASP) Subcommitttee on Taxonomy. Pain, 6, 249-252.

Mount, B.M. (1984). Psychological and social aspects of cancer pain. Dans P.D. Wall et R. Melzack (Eds.) Texbook of pain (pp. 460- 47I). Edinburg: Churchill Livingstone.

Mouren-Mathieu, A.M. (1987). Soins palliatifs. Montréal: Les Presses de l'Université de Montréal.

Myers, D. (1992). Psychology (3e ed.). Worth. Traduction espagnole: Psicología. Madrid: Panamericana

Razavi, D. et Delvaux, N. (1994). Psycho-oncologie Paris: Masson.

Sanz, J., Gómez-Batiste, X., Gómez Sancho, M. et Núñez Olarte, J.M. (1993). Cuidados paliativos: Recomendaciones de la Sociedad Española de Cuidados Paliativos (SECPAL). Madrid: Ministerio de Sanidad y Consumo.

Stedeford, A. (1984). Facing death: patients, families and professionals. Oxford: Heinemann.

Vachon, M.L.S. (1993). Emotional problems in palliative medicine: patient, family, and professional. Dans D. Doyle, G.W.C. Hanks et N. MacDonals (Eds.) Oxford textbook of palliative medicine (pp. 577-605). Oxford: Oxford University Press. 
Série

Investigaçāo

Coimbra

Imprensa da Universidade

Les Presses de L'Université Laval

Les Editions de L'IORC

2004 